\title{
Estratégias de resolução para o problema de job-shop flexível
}

\author{
Wellington Donizeti Previero
}

TESE APRESENTADA

$\mathrm{AO}$

Instituto DE MATEmÁticA E EstatísticA

DA

Universidade DE SÃo PAulo

PARA

OBTENÇÃO DO TÍTULO

$\mathrm{DE}$

Doutor EM CiênCIAS

\author{
Programa: Ciência da Computação \\ Orientador: Prof. Dr. Carlos Eduardo Ferreira
}

Durante o desenvolvimento deste trabalho o autor

recebeu auxílio financeiro da Fundação Araucária 



\section{Estratégias de resolução para o problema de job-shop flexível}

Esta versão da tese contém as correções e alterações sugeridas pela Comissão Julgadora durante a defesa da versão original do trabalho, realizada em 16/09/2016. Uma cópia da versão original está disponível no Instituto de Matemática e Estatística da Universidade de São Paulo.

Comissão Julgadora:

- Prof ${ }^{a}$. Dr. Carlos Eduardo Ferreira (Orientador) - IME-USP

- Prof. Dr. Aritanan Borges Garcia Gruber - IME-USP

- Prof. Dra ${ }^{a}$ Debora Pretti Ronconi - EP-USP

- Prof. Dr. Nelson Maculan Filho - UFRJ

- Prof. Dr. Silvio Alexandre de Araujo - IBILCE-UNESP 



\section{Agradecimentos}

Ao professor Carlinhos pela orientação, amizade, paciência, disponibilidade e espírito motivador.

Ao professor Silvio Alexandre de Araujo pela atenção e pelos valorosos conselhos.

Aos meus pais, José Carlos e Maria, e ao meu irmão Leandro.

À todos os professores que contribuíram para a minha formação profissional e pessoal, desde o ínicio na E.E Laurindo Battaiola (Barra Bonita-SP), até o presente momento.

Aos meus colegas do DINTER: Alessandro Kraemer, André Luiz Satoshi Kawamoto, André Schwerz, Francisco Pereira Junior, Frank Helbert Borsato, Igor Fábio Steinmacher, Igor Wiese, Ivanilton Polato, Lucio Geronimo Valentin, Luiz Arthur Feitosa dos Santos, Marcos Silvano Almeida, Rafael Liberato Roberto, Rodrigo Campiolo e Rogério Aparecido Gonçalves.

À UTFPR, câmpus Londrina, e aos colegas do Departamento Acadêmico de Matemática pelo apoio.

Em especial, a minha esposa Paula, pelo carinho, amor, paciência (e muita) e companheirismo (até mesmo nas correções). Um enorme beijo!!

Este trabalho não seria possível sem o projeto de Doutorado Interinstitucional (DINTER) entre a Universidade Tecnológica Federal do Paraná - UTFPR, câmpus Campo Mourão, e o Instituto de Matemática e Estastística - IME/USP. Meus agradecimentos aos professores Fabio Kon e Carlos Eduardo Ferreira por apoiarem e abraçarem o projeto e ao professor Reginaldo Ré pela coordenação das atividades.

À Fundação Araucária, Departamento de Ciência, Tecnologia e de Ensino Superior do Estado do Paraná (SETI-PR) e o Governo do Estado do Paraná pelo financiamento que viabilizou o DINTER. 



\section{Resumo}

PREVIERO, W. D. Estratégias de resolução para o problema de Job-Shop Flexível. 2016. 134 f. Tese (Doutorado) - Instituto de Matemática e Estatística, Universidade de São Paulo, São Paulo, 2016.

Nesta tese apresentamos duas estratégias para resolver o problema de job-shop flexível com o objetivo de minimizar o makespan. A primeira estratégia utiliza um algoritmo branch and cut (B\&C) e a segunda abordagens matheuristics. O algoritmo B\&C utiliza novas classes de inequações válidas, originalmente formulada para o problema de job-shop e estendida para o problema em questão. Para que as inequações válidas sejam eficientes, o modelo proposto por Birgin et al, (2014) (A milp model for an extended version of the fexible job shop problem. Optimization Letters, Springer, v. 8, n. 4, 1417-1431), é reformulado (MILP-2). A segunda estratégia utiliza as matheuristcs local branching e diversification, refining and tight-refining. Os experimentos computacionais mostraram que a inclusão dos planos de corte melhoram a relaxação do modelo MILP-2 e a qualidade das soluções. O algoritmo B\&C reduziu o gap e o número de nós explorados para uma grande quantidade de instâncias. As abordagens matheuristics tiveram um excelente desempenho. Do total de 59 instâncias analisadas, somente em 3 problemas a resolução do modelo MILP-1 obteve melhores resultados do que as abordagens matheuristcs.

Palavras-chave: job-shop flexível, inequações válidas, branch and cut, matheuristcs. 



\section{Abstract}

PREVIERO, W. D. Solution approaches for flexible job-shop scheduling problem. 2016. 134 f. Tese (Doutorado) - Instituto de Matemática e Estatística, Universidade de São Paulo, São Paulo, 2016.

This thesis proposes two approaches to solve the flexible job-shop scheduling problem to minimize the makespan. The first strategy uses a branch and cut algorithm (B\&C) and the second approach is based on matheuristics. The B\&C algorithm uses new classes of valid inequalities, originally formulated for job-shop scheduling problems and extended to the problem at hand. The second approach uses the matheuristics local branching and diversification, refining and tight-refining. For all valid inequalities to be effective, the precedence variable based model proposed by Birgin et al, (2014) (A milp model for an extended version of the fexible job shop problem. Optimization Letters, Springer, v. 8, n. 4, 1417-1431), is reformulated (MILP-2). The computational experiments showed that the inclusion of cutting planes tightened the linear programming relaxations and improved the quality of solutions. B\&C algorithm reduced the gap value and the number of nodes explored in a large number of instances. The matheuristics approaches had an excellent performance. From 59 instances analized, MILP-1-Gurobi showed better results than matheuristics approaches in only 3 problems.

Keywords: flexible job-shop, valid inequalities, branch and cut, matheuristics. 



\section{Lista de ilustrações}

Figura 1 - Diagrama de Gantt de uma solução factível para o JS - Solução 1. . . . . 37

Figura 2 - Diagrama de Gantt de uma solução factível para o JS - Solução 2. . . . . 37

Figura 3 - Grafo disjuntivo para o JS descrito pela Tabela 1. . . . . . . . . . . . 39

Figura 4 - Representação da seleção completa e consistente das soluções 1 e 2. . . . 39

Figura 5 - Representação do caminho crítico para as soluções 1 e 2. . . . . . . . . 40

Figura 6 - Representação das soluções 1 e 2 sem arcos redundantes. . . . . . . . . . 41

Figura 7 - Blocos do caminho crítico. . . . . . . . . . . . . . . 42

Figura 8 - Grafo disjuntivo associado a atribuição $\mu$. . . . . . . . . . . . . 49

Figura 9 - Exemplo do Grafo 3-Diciclo. . . . . . . . . . . . . . . . . 62

Figura 10 - Exemplo do Grafo 3-Fence. As linhas pontilhadas representam os arcos estacas e as linhas contínuas os arcos piquetes. . . . . . . . . . 63

Figura 11 - Diagrama de Gantt de uma solução factível para a instância sfjs01. . . 67

Figura 12 - Esquema do método LB tradicional. . . . . . . . . . . . . . . . . 97

Figura 13 - Esquema do método LB com limite de tempo: solução incumbente atualizada 98

Figura 14 - Esquema local branching com limite de tempo: solução incumbente não atualizada . . . . . . . . . . . . . . . . . . . . . 99

Figura 15 - Esquema local branching com estratégia de diversificação . . . . . . . . . 100

Figura 16 - Evolução do limitante superior da instância 02a do conjunto DPdata. . . 101

Figura 17 - Evolução do limitante superior da instância MK09 do conjunto BRdata . 107

Figura 18 - Etapa de calibração do parâmetro k . . . . . . . . . . . . . . . 110

Figura 19 - Etapa de calibração do parâmetro $t_{l e f t} \ldots \ldots \ldots \ldots$. . . . . . . . 111

Figura 20 - Resolução da instância 02a do conjunto DPdata . . . . . . . . . . . . . 115 



\section{Lista de tabelas}

Tabela 1 - Exemplo de uma instância do JS com três tarefas e três operações. . . 37

Tabela 2 - Técnicas utilizadas para a resolução do JSF . . . . . . . . . . . . 46

Tabela 3 - Instância para o JSF com 3 tarefas e 3 máquinas. . . . . . . . . . . . . . 48

Tabela 4 - Dados da instância sfjs01 de Fattahi, Mehrabad e Jolai (2007). . . . . . . 67

Tabela 5 - Valores para as variáveis $s_{i}, p_{i}$ e $c_{i}$ na solução factível da instância sfjs01 de Fattahi, Mehrabad e Jolai (2007). . . . . . . . . . . . . . . 67

Tabela 6 - Descrição do conjunto Fdata e do número de variáveis e restrições dos modelos MILP-1 e MILP-2. . . . . . . . . . . . . . . . . . . 73

Tabela 7 - Descrição do conjunto BRdata e do número de variáveis e restrições dos modelos MILP-1 e MILP-2. . . . . . . . . . . . . . . . . . 74

Tabela 8 - Descrição da construção dos problemas do conjunto BCdata. . . . . . . . 74

Tabela 9 - Descrição do conjunto BCdata e do número de variáveis e restrições dos modelos MILP-1 e MILP-2. . . . . . . . . . . . . . . . . . 74

Tabela 10 - Descrição do conjunto DPdata e do número de variáveis e restrições dos modelos MILP-1 e MILP-2. . . . . . . . . . . . . . . . . .

Tabela 11 - Valores mínimos, máximos e médios do número de variáveis binárias, contínuas e restrições para o modelo MILP-1. . . . . . . . . . . . . 76

Tabela 12 - Resultado do algoritmo de planos de corte para o conjunto Fdata. . . . . 78

Tabela 13 - Resultado do algoritmo de planos de corte para o conjunto BRdata. . . . 79

Tabela 14 - Resultado do algoritmo de planos de corte para o conjunto BCdata. . . . 80

Tabela 15 - Resultado do algoritmo de planos de corte para o conjunto DPdata. . . . 81

Tabela 16 - Resultado do algoritmo de planos de corte para os grupos 1 e 2. . . . . 81

Tabela 17 - Resultado do algoritmo de planos de corte para os grupos 3 e 4 . . . . . 82

Tabela 18 - Valor da média dos gaps para cada combinação dos parâmetros $k$ e $f$ no conjunto para as 10 instâncias de teste. . . . . . . . . . . . . . 84

Tabela 19 - Resultados computacionais de MILP-1-Gurobi, MILP-2-Gurobi e MILP-2B\&C para o conjunto Fdata. . . . . . . . . . . . . . . 85

Tabela 20 - Resultados computacionais de MILP-1-Gurobi, MILP-2-Gurobi e MILP-2B\&C para o conjunto BRdata. . . . . . . . . . . . . . . . . .

Tabela 21 - Resultados computacionais de MILP-1-Gurobi, MILP-2-Gurobi e MILP-2B\&C para o conjunto BCdata. . . . . . . . . . . . . 87

Tabela 22 - Resultados computacionais de MILP-1-Gurobi, MILP-2-Gurobi e MILP-2B\&C para o conjunto DPdata. . . . . . . . . . . . . . . .

Tabela 23 - Resumo dos resultados para cada conjunto de instâncias teste. Os valores representam a média para cada conjunto.

Tabela 24 - Média das inequações violadas e da porcentagem destinada ao tempo da etapa de separação de MILP-2-B\&C. . . . . . . . . . . . . . . . . .

Tabela 25 - Resumo dos resultados para cada conjunto de instâncias teste. Os valores representam a média para cada conjunto. . . . . . . . . . . . . 89

Tabela 26 - Resultados computacionais de MILP-2-B\&C para as instâncias em aberto. 91

Tabela 27 - Exemplo da etapa de calibração no instante $t=100$ segundos. . . . . . . 109

Tabela 28 - Resultados computacionais de MILP-1-Gurobi, LB_SD e LB_CD para o conjunto Fdata. . . . . . . . . . . . . . . . . . . . . . . . . . . . . . . . . 112

Tabela 29 - Resultados computacionais de MILP-1-Gurobi, LB_SD e LB_CD para o conjunto BRdata. . . . . . . . . . . . . . . . 112 
Tabela 30 - Resultados computacionais de MILP-1-Gurobi, LB_SD e LB_CD para o conjunto BCdata. . . . . . . . . . . . . . . . . . . . . 113

Tabela 31 - Resultados computacionais de MILP-1-Gurobi, LB_SD e LB_CD para o conjunto DPdata. . . . . . . . . . . . . . . . . . . . . 114

Tabela 32 - Valor da média do GAP para cada procedimento e estratégia de obtenção da solução inicial . . . . . . . . . . . . . . . . . . . . . . 115

Tabela 33 - Resultados computacionais de MILP-1-Gurobi, DRT1 e DRT2 para o conjunto Fdata. . . . . . . . . . . . . . . . . . . . . . . . . . . 119

Tabela 34 - Resultados computacionais de MILP-1-Gurobi, DRT1 e DRT2 para o conjunto BRdata. . . . . . . . . . . . . . . . . . . . . 119

Tabela 35 - Resultados computacionais de MILP-1-Gurobi, DRT1 e DRT2 para o conjunto BCdata. . . . . . . . . . . . . . . . . . . 120

Tabela 36 - Resultados computacionais de MILP-1-Gurobi, DRT1 e DRT2 para o conjunto DPdata. . . . . . . . . . . . . . . . . 121

Tabela 37 - Resultados computacionais de DRT1-Big. . . . . . . . . . . . . . . 121

Tabela 38 - Melhores resultados para as instâncias do conjunto Fdata . . . . . . . . . 122

Tabela 39 - Melhores resultados para as instâncias do conjunto BRdata . . . . . . . 122

Tabela 40 - Melhores resultados para as instâncias do conjunto BCdata . . . . . . . 123

Tabela 41 - Melhores resultados para as instâncias do conjunto DPdata . . . . . . . 124 


\title{
Lista de abreviaturas e siglas
}

\author{
MILP Problema de programação linear inteira mista \\ LP Problema de programação linear \\ IP Problema de programação inteira \\ BP Problema de programação binária \\ COP Problema de otimização combinatória \\ PC Planos de corte \\ $B \& B \quad$ Branch and bound \\ $B \& C \quad$ Branch and cut \\ POL Problema de ordenação linear \\ LB $\quad$ Local branching \\ DRT Diversification, refining and tight-refining
}





\section{Sumário}

Contribuições . . . . . . . . . . . . . . . . . . . . . . . . . . . . . 19

Organização do trabalho . . . . . . . . . . . . . . . . . . . . . . 19

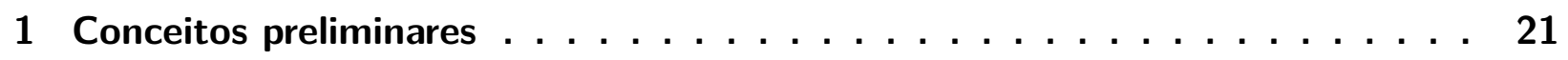

1.1 Resolvedores . . . . . . . . . . . . . . . . . . . . . . . . . . 21

1.2 Problemas de otimização . . . . . . . . . . . . . . . . . . . . . . 22

1.3 Teoria poliédrica e inequações válidas . . . . . . . . . . . . . . . 23

1.4 Métodos exatos . . . . . . . . . . . . . . . . . . . . . 25

1.4.1 Algoritmo branch and bound . . . . . . . . . . . . . 26

1.4.2 Algoritmo de planos de corte . . . . . . . . . . . . . . . . 27

1.4.3 Algoritmo branch and cut . . . . . . . . . . . . . . . 28

1.5 Heurísticas . . . . . . . . . . . . . . . . . . . . . . 30

1.5 .1 Metaheurística . . . . . . . . . . . . . . . . 30

1.5.1.1 Busca Local . . . . . . . . . . . . . . . . 30

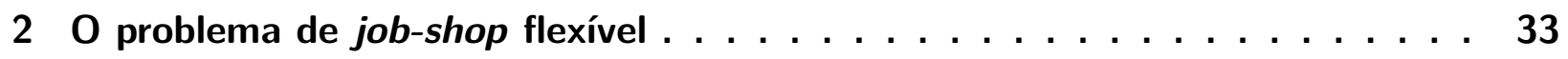

2.1 O problema de programação de tarefas . . . . . . . . . . . . . 33

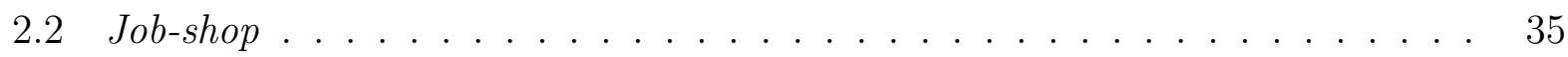

2.2.1 Descrição do job-shop e formulação matemática . . . . . . . . . 36

2.3 Job-shop flexível . . . . . . . . . . . . . . . . . . . . . . . . 43

2.3.1 Descrição do job-shop flexível e formulação matemática (MILP-1) . . 47

2.4 Resumo . . . . . . . . . . . . . . . . . . . . . . . . 52

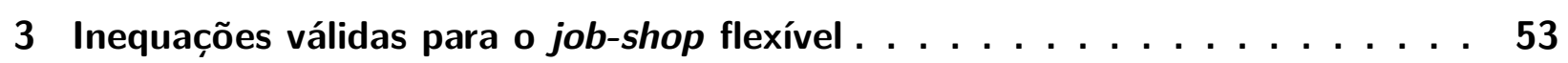

3.1 Inequações válidas para o job-shop . . . . . . . . . . . . . . 53

3.2 Inequações válidas para job-shop flexível . . . . . . . . . . . . . 60

3.3 Reformulação do modelo MILP-1 (MILP-2) . . . . . . . . . . . . 66

3.3.1 Comparação entre os modelos MILP-1 e MILP-2 . . . . . . . . . . . 69

3.4 Problema de separação . . . . . . . . . . . . . . . . . . . . . . . . 70

3.5 Experimentos computacionais . . . . . . . . . . . . . . . . 71

3.5.1 Conjunto de instâncias teste . . . . . . . . . . . . 71

3.5.1.1 Conjunto Fdata . . . . . . . . . . . . . . . . . 72

3.5.1.2 Conjunto BRdata. . . . . . . . . . . . . . . . 72

3.5.1.3 Conjunto BCdata. . . . . . . . . . . . . . . . 72

3.5.1.4 Conjunto DPdata. . . . . . . . . . . . . . . 75

3.5.1.5 Breve resumo dos conjuntos de instâncias teste $\ldots \ldots . .76$

3.5.2 Resultados do algoritmo de planos de corte . . . . . . . . . . 76

3.5.3 Resultados do algoritmo branch and cut . . . . . . . . . . . 80

3.5.3.1 Instâncias em aberto . . . . . . . . . . . . . . . . . . 89 


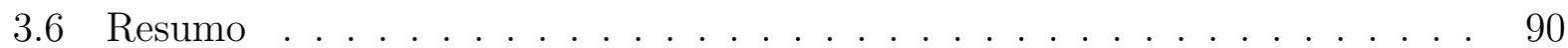

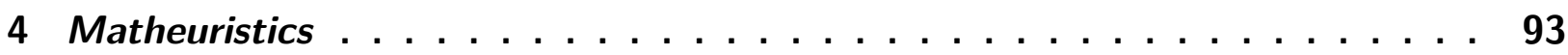

4.1 Introdução . . . . . . . . . . . . . . . . . . . . . . . . . . 93

4.2 Local branching . . . . . . . . . . . . . . . . . . . . . . 95

4.3 Diversification, refining, and tight-refining . . . . . . . . . . . 103

4.4 Experimentos computacionais . . . . . . . . . . . . . . . 107

4.4.1 Experimentos computacionais para o método local branching . . . . . 108

4.4.2 Experimentos computacionais para método DRT . . . . . . . . . . 116

4.4.3 Síntese dos experimentos para os métodos local branching e DRT . . . 122

4.5 Resumo . . . . . . . . . . . . . . . . . . . 123

5 Conclusão . . . . . . . . . . . . . . . . . . . . . 125

5.1 Considerações finais . . . . . . . . . . . . . . . . . . . . . 125

5.2 Trabalhos futuros . . . . . . . . . . . . . . . . . 126

Referências .......................... . 127 


\section{Introdução}

O problema de programação de tarefas, conhecido na língua inglesa como scheduling, é um processo importante de tomada de decisão presente na maioria dos sistemas de manufatura e produção. No problema devemos alocar recursos, geralmente limitados, a atividades a serem realizadas. Os problemas oriundos oferecem um grande desafio teórico para os pesquisadores por causa de sua natureza combinatória.

O job-shop (JS) é um problema importante da área de scheduling e descreve uma ampla variedade de aplicações. O JS é composto por um conjunto de máquinas que executam as operações de uma tarefa. Cada tarefa tem uma ordem de processamento específica através nas máquinas, isto é, uma tarefa é composta por uma lista ordenada de operações. O objetivo do JS é definir uma sequência de processamento das operações em cada máquina que otimize uma medida de desempenho.

Nos dias de hoje, em decorrência do avanço tecnológico e das necessidades do mercado, máquinas com múltiplas funções estão cada vez mais presente nas indústrias. O job-shop flexível (JSF), problema abordado neste trabalho, surge neste contexto.

Ao contrário do JS, no JSF diferentes máquinas estão habilitadas para processar uma ou várias operações que compõem cada tarefa. Dessa forma, com o objetivo de otimizar uma medida de desempenho, a resolução do JSF pode ser dividido em dois subproblemas: roteamento e sequenciamento. O subproblema de roteamento é caracterizado por atribuir cada operação a uma máquina específica e o subproblema de sequenciamento por definir a ordem de execução das operações em cada máquina.

Portanto, o JSF é mais complexo que o JS devido à necessidade adicional de determinar um roteiro de atribuições para as operações. O JSF é um problem NP-difícil, pois é uma extensão do JS (MASTROLILLI; GAMBARDELLA, 2000). Além disso, de acordo com Lawler et al. (1993), o JSF é considerado como um dos problemas mais difíceis de otimização combinatória. Dentre os principais conjuntos de instâncias teste para o JSF, ainda há várias instâncias para as quais não se conhece uma solução ótima, como por exemplo a instância 05a, de Dauzère-Pérès e Paulli (1997), composta por apenas 10 tarefas e 5 máquinas.

Os principais métodos exatos encontrados na literatura para o JSF são baseados na utilização de pacotes comerciais de otimização em formulações lineares, não lineares de programação inteira mista e programação por restrições (constraint programming). Embora os métodos exatos não possam fornecer uma solução eficiente para o JSF, eles podem ser o primeiro passo antes de desenvolver heurísticas eficazes e úteis para a compreensão da estrutura do problema (DEMIR; İşLEYEN, 2013). Métodos exatos podem ser capazes de fornecer, senão uma solução ótima, limitantes satisfatórios para muitos problemas, além de avaliar a qualidade de métodos heurísticos.

Dessa forma, com o intuito de contribuir com novas abordagens, estabelecemos como 
o foco principal desta tese o uso de métodos exatos para a resolução do JSF por meio de um modelo de programação linear inteira mista. A medida de desempenho que utilizamos é minimizar o makespan, isto é, o tempo de conclusão da última tarefa no sistema.

O problema de programação de tarefas em uma única máquina (single machine scheduling) é um dos problemas mais simples na área de scheduling. As abordagens utilizadas em sua resolução proporcionam uma base para a concepção de técnicas em ambientes mais complexos, como por exemplo, o JS. Em muitos casos, em decorrência da complexidade, problemas de programação com várias máquinas são decompostos em subproblemas que tratam com máquinas individuais. Por exemplo, o trabalho de Balas (1985) apresenta uma descrição parcial do envoltório convexo do conjunto das soluções factíveis para o JS. O autor focou a atenção para o problema em uma única máquina e estendeu os resultados obtidos para o JS. Em especial, a pesquisa de Applegate e Cook (1991) apresenta um algoritmo de planos de corte para JS e a maioria das inequações válidas utilizadas são as mesmas descritas por outros autores para o problema de programação de tarefas em uma única máquina. Dentro do contexto de estender técnicas para o ambiente de várias máquinas, inicialmente identificamos que não havia na literatura nenhum trabalho que fizesse a transição do uso das inequações válidas do JS ou do problema de uma única máquina para o ambiente do JSF. Dessa forma, a primeira estratégia de resolução do JSF baseia-se no uso de classes de inequações válidas em um algoritmo exato, como o branch and cut.

Em decorrência da complexidade do JSF, muitas vezes é preferível sacrificar a obtenção da solução ótima com alto esforço computacional em favor de obter uma solução razoável em um período de tempo mais curto. Dessa forma, as abordagens heurísticas e metaheurísticas representam as principais estratégias de resolução presentes na literatura.

Embora métodos exatos e (meta)heurísticos pertençam a duas categorias distintas, muitas de suas técnicas possuem uma estrutura comum e que permitem um certo grau de unificação (HOOKER, 2015). A sinergia entre estas duas abordagens permite agregar, em um único método, a robustez e a eficácia que caracterizam as metaheurísticas, com o poderio dos pacotes comerciais de otimização e das técnicas de resolução de métodos exatos.

Tais abordagens híbridas foram intituladas pela comunidade científica de matheuristics ou modelos baseados em metaheurísticas e tornaram-se populares ao longo das duas últimas décadas. Segundo Boschetti et al. (2009), matheuristics são algoritmos obtidos pela interrelação de técnicas de programação matemática e metaheurística. A combinação entre as duas técnicas pode ocorrer de duas maneiras: a programação matemática como uma aliada para orientar ou personalizar metaheurísticas ou utilizar os conceitos e técnicas das metaheurísticas em métodos de programação matemática.

O JS tem recebido bastante atenção dos pesquisadores em relação ao uso das matheuristics. Fernandes e Lourenço (2007) apresentam uma listagem de abordagens que utilizam a busca local com métodos exatos e o JS aparece como sendo o problema com o maior número de aplicações. Entretanto, o mesmo não ocorre com JSF.

As abordagens híbridas utilizadas para a resolução do JSF são de natureza metaheurística, isto é, obtidas pela combinação de dois ou mais algoritmos metaheurísticos. Desta forma, nos sentimos motivados em aplicar e avaliar algumas das abordagens matheuristics no JSF.

Com base nessas considerações, apresenta-se nesta tese um conjunto de inequações válidas para o JSF e a implementação de duas abordagens matheuristics. Duas classes de inequações são obtidas através do problema de ordenação linear e seis classes do JS. Todas as 
classes foram avaliadas através de um algoritmo de planos de corte, e as de melhor desempenho foram utilizadas em algoritmo branch and cut. Os métodos matheuristics implementados nesta tese são as técnicas local branching e diversification, refining and tight-refining. Os resultados obtidos pelo algoritmo branch and cut e pelas matheuristics foram comparados com a melhor formulação disponível na literatura para o problema.

\section{Contribuições}

As principais contribuições deste trabalho são as seguintes:

- As classes de inequações derivadas do JS representam novos conjuntos de cortes para JSF, considerando o modelo de programação linear inteira mista com variáveis de precedência. Além disso, embora as demais classes utilizadas (3 diciclos e 3-fences) sejam originalmente propostas para o problema de ordenação linear, estas não tinham sido avaliadas para o caso do JSF.

- Aplicação das matheuristics local branching e diversification, refining and tight-refining na resolução do JSF. Uma versão do método diversification, refining and tight-refining foi implementada levando em consideração algumas propriedades estruturais do problema.

\section{Organização do trabalho}

Esta tese está organizada em 5 capítulos. Os tópicos a serem cobertos em cada capítulo são descritos na sequência.

O Capítulo 1 apresenta um conjunto de conceitos que servem como base teórica para esta tese e que serão úteis para um melhor entendimento do trabalho. São abordados os temas referentes a resolvedores, os diversos problemas de programação, teoria poliedral e heurísticas e metaheurísticas. São abordados também os algoritmos branch and bound, planos de corte e branch and cut.

O Capítulo 2 descreve os problemas de JS e JSF. Para ambos os problemas são apresentados as suas características, algumas formulações e propriedades. Além disso, o capítulo discute os principais trabalhos relacionados que utilizam modelos de programação linear inteira mista para a resolução do problema.

As inequações válidas são descritas no Capítulo 3. O Capítulo também apresenta os conjuntos de instâncias teste utilizados nos experimentos computacionais e os resultados obtidos pelos algoritmos de planos de corte e branch and cut. O algoritmo de planos de corte é utilizado para verificar a qualidade do limitante inferior após o acréscimo das classes de inequações e identificar se existe alguma família com melhor desempenho comparada com as demais.

O Capítulo 4 traz os conceitos relacionados a matheuristics e as duas abordagens utilizadas nos experimentos computacionais: local branching e diversification, refining and tight-refining. O Capítulo 5 conclui o trabalho com as considerações finais e trabalhos futuros. 



\section{Capítulo 1 Conceitos preliminares}

Neste capítulo, abordamos, de forma resumida, diversos dos conceitos presentes nesta tese. Inicialmente apresentamos o conceito de resolvedores para problemas de otimização e diversos tipos de problemas de programação matemática. Em seguida, são apresentadas as principais definições e propriedades de teoria poliédrica e três abordagens clássicas para resolver problemas de linear inteira: branch and bound, planos de corte e branch and cut. O capítulo é finalizado expondo as definições relacionadas aos métodos heurísticos e metaheurísticos. Utilizamos como referências os livros de Wolsey e Nemhauser (1988), Ferreira e Wakabayashi (1996), Pochet e Wolsey (2006) e Talbi (2009).

\subsection{Resolvedores}

Neste trabalho, o termo resolvedor é utilizado para expressar uma ferramenta computacional destinada à solução de problemas de otimização. Um resolvedor pode ser um programa de computador ou uma biblioteca de algum software matemático. Dentre os principais resolvedores, destacamos o Gurobi (GUROBI, 2016), o Cplex (CPLEX, 2016) e o Xpress (XPRESS, 2016).

Embora os três citados sejam comerciais, para o Gurobi e o Cplex há duas versões gratuitas, uma destinada ao público geral, com limitação no número de variáveis e restrições, e outra destinada à comunidade acadêmica e sem limitações nas dimensões do modelo. O Xpress tem uma versão acadêmica, gratuita e com limitação no número de variáveis e outra gratuita e irrestrita ao tamanho, mas limitado ao uso pelo período de 30 dias. Na categoria de resolvedores de código aberto ou de licença não comercial, Linderoth e Ralphs (2005) apresentam uma descrição detalhada de oito resolvedores específicos para problemas de programação linear inteira mista.

Utilizamos o Gurobi para resolver os problemas de otimização em todos os experimentos computacionais. O nome Gurobi é um acrônimo do sobrenome de seus fundadores: Zonghao Gu, Edward Rothberg e Robert Bixby. Anteriormente à criação do Gurobi, Robert Bixby também foi um dos fundadores do Cplex, enquanto Zonghao Gu e Edward Rothberg lideraram equipes de desenvolvimento do Cplex. 


\subsection{Problemas de otimização}

Um problema de programação linear inteira mista (MILP) é uma classe de problema de programação matemática no qual desejamos encontrar um conjunto de valores para as variáveis reais (ou contínuas) e inteiras, que maximiza ou minimiza uma função objetivo linear e que satisfaz um conjunto de restrições lineares. Um MILP pode ser expresso por

$$
\begin{aligned}
& \left(P_{M I L P}\right) \quad z_{M I L P}=\text { Minimizar } c x \\
& \text { Sujeito a } A x \leq b \text {, } \\
& x_{j} \in \mathbb{Z}_{+}, \quad \forall j \in \mathscr{Z} \\
& x_{j} \in \mathbb{R}_{+}, \quad \forall j \in \mathscr{C}
\end{aligned}
$$

em que os elementos dos conjuntos $\mathscr{Z}$ e $\mathscr{C}$ representam os índices das variáveis inteiras e reais, respectivamente. Sendo que $n=|\mathscr{Z}|+|\mathscr{C}|$, a matriz $A(m \times n)$ e os vetores $c(1 \times n)$ e $b$ $(n \times 1)$ representam os parâmetros do problema. Por simplicidade, assumimos que $A x \leq b \neq \emptyset$ e $z_{M I L P}>-\infty$.

Segundo o livro de Chen, Batson e Dang (2010), o termo programação, no contexto de problemas de otimização, denota o planejamento de atividades que necessitam de recursos e que atendem às exigências impostas. Os termos programação e otimização são utilizados indistintamente por Arenales et al. (2007, p.-163). Nos últimos anos a comunidade de Otimização tem abandonado o termo "programação".

Dado o problema $P_{M I L P}$, o conjunto $X=\left\{x_{j}, j \in \mathscr{Z} \cup \mathscr{C} ; A x \leq b\right\}$ é denominado de conjunto das soluções factíveis ou das soluções viáveis. A solução factivel $x^{*}$ tal que $z_{M I L P}=c x^{*}$ é denonimada de solução ótima.

Quando todas as variáveis de $P_{M I L P}$ são contínuas, isto é, $\mathscr{Z}=\emptyset$, tem-se um problema de programação linear (LP). Se todas as variáveis são inteiras, então tem-se um problema de programação inteira (IP), e ainda, se as variáveis inteiras assumem valores 0 ou 1, o problema resultante representa um problema de programação 0-1 ou binário (BP).

Em um problema de otimização combinatória (COP), é dado um conjunto $N=$ $\{1,2, \ldots, n\}$, um vetor peso $c=\left(c_{1}, c_{2}, \ldots, c_{n}\right)$ e uma família $\mathscr{F} \subseteq 2^{|N|}$ de subconjuntos factíveis de $N$. O objetivo do COP é resolver

$$
\operatorname{Minimizar}\left\{\sum_{j \in S} c_{j} ; S \in \mathscr{F}\right\} \text {. }
$$

Dessa forma, em um COP busca-se determinar um subconjunto $S$ de $\mathscr{F}$ tal que o seu custo associado seja mínimo. Muitos COPs podem ser formulados por MILPs ou BPs.

As técnicas para resolver COPs podem ser divididas em duas categorias: métodos exatos e métodos aproximados. Métodos exatos garantem encontrar uma solução e provar a sua otimalidade para qualquer instância de um COP. Entretanto, o tempo de computação frequentemente cresce com o tamanho do problema e, em muitos casos, uma parte significativa deste tempo é atribuída para provar que a solução encontrada é ótima. Ao considerar métodos exatos, as seguintes técnicas são de grande importância na literatura: programação dinâmica, programação por restrições (constraint programming) e métodos baseados em relaxações, tais como algoritmo de planos de corte, geração de colunas, branch and bound, branch and cut, branch and price e branch and cut and price. 
Na categoria de métodos aproximados, duas subclasses de algoritmos podem ser encontradas: algoritmos de aproximação e algoritmos heurísticos. Algoritmos de aproximação e heurísticos são técnicas que buscam boas soluções a um custo computacional razoável, sem dispor de garantias que a solução ótima seja obtida. Ao contrário dos algoritmos de aproximação, algoritmos heurísticos não fornecem garantias de quão próxima a solução encontrada está do valor ótimo (RAYWARD-SMITH et al., 1996).

\subsection{Teoria poliédrica e inequações válidas}

Considere o IP descrito por

$$
\begin{aligned}
& \left(P_{I P}\right) \quad z_{I P}=\text { Minimizar } c x \\
& \text { Sujeito a } A x \leq b \text {, } \\
& x \in \mathbb{Z}_{+}^{n} \text {, }
\end{aligned}
$$

em que $A$ e $b$ são matrizes de dimensões $(m, n)$ e $(m, 1)$, respectivamente, e coeficientes racionais. Seja $X$ o seu conjunto de soluções factíveis, isto é, $X=\left\{x \in \mathbb{Z}_{+}^{n} ; A x \leq b\right\}$.

Embora as discussões e definições desta seção sejam baseadas para o problema $P_{I P}$, a teoria é igualmente válida para todo MILP.

Definição 1. Um subconjunto $P \subseteq \mathbb{R}^{n}$ é chamado de poliedro se

$$
P=\left\{x \in \mathbb{R}^{n}: A x \leq b\right\}
$$

para alguma matriz $A \in \mathbb{R}^{m \times n}$ e algum vetor $b \in \mathbb{R}^{m}$.

De acordo com a definição 1 , um poliedro $P$ é um conjunto de pontos que satisfaz uma quantidade finita de inequações lineares. Um poliedro é denominado de racional quando existe uma matriz $A^{\prime} \in \mathbb{R}^{m \times n}$ e algum vetor $b^{\prime} \in \mathbb{R}^{m}$ com coeficientes racionais tal que $P=\left\{x \in \mathbb{R}^{n}: A^{\prime} x \leq b^{\prime}\right\}$. Um poliedro $P \subseteq \mathbb{R}^{n}$ é limitado se existe um número real $\alpha \in \mathbb{R}_{+}$ tal que todo $x \in P$ satisfaz $\|x\| \leq \alpha$. Um poliedro limitado é denominado politopo.

Sejam $M=\{1,2, \ldots, m\}, M^{=}=\left\{i \in M: A^{i} x=b_{i}, \forall x \in P\right\}$ e $M \leq=\{i \in M:$ $\left.A^{i} x<b_{i}, \forall x \in P\right\}$. Conforme a notação, o conjunto $M^{=}$representa os índices das linhas do sistema $A x \leq b$, cujas respectivas inequações são satisfeitas com igualdade por todos os pontos do poliedro $P$ e $M^{\leq}=M \backslash M^{=}$. Considere também $\left(A^{=}, b^{=}\right)$e $\left(A^{\leq}, b^{\leq}\right)$como sendo as linhas da matriz $(A, b)$ associadas aos conjuntos $M^{=}$e $M^{\leq}$, respectivamente.

Definição 2. Um poliedro $P$ é uma formulação para $X$, se $X=P \cap \mathbb{Z}_{+}^{n}$.

A definição acima estabelece que, se $P$ é uma formulação para $X$, então $X$ corresponde ao conjunto de pontos inteiros de $P$.

Sejam $P^{1}$ e $P^{2}$ duas formulações para $X$, tais que $P^{1} \subset P^{2}$. Então, temos que

$$
z_{I P} \geq \min \left\{c x ; x \in P^{1}\right\} \geq \min \left\{c x ; x \in P^{2}\right\} \text {. }
$$

Dessa forma, como o conjunto $X$ pode ter infinitas formulações, dizemos que a formulação $P^{1}$ é melhor que a formulação $P^{2}$ se $P^{1} \subset P^{2}$. 
Definição 3. Seja $S \subseteq \mathbb{R}^{n}$. Um ponto $x \in \mathbb{R}^{n}$ é uma combinação convexa de pontos do conjunto $S$ se existe um número finito de pontos $\left\{x^{i}\right\}_{i=1}^{t} \in S$ e $\lambda \in \mathbb{R}_{+}^{t}$ tal que $x=\sum_{i=1}^{t} \lambda_{i} x^{i}$, $\operatorname{com} \sum_{i=1}^{t} \lambda_{i}=1$. O envoltório convexo de $S$, denotada por conv $(S)$, é o conjunto de todos os pontos que podem ser escritos como uma combinação convexa de pontos de $S$, isto é,

$$
\operatorname{conv}(S)=\left\{x=\sum_{i=1}^{t} \lambda_{i} x^{i}: \sum_{i=1}^{t} \lambda_{i}=1, x^{i} \in S, \lambda_{i} \in \mathbb{R}_{+} e i=1, \cdots, t\right\} .
$$

Para IPs e MILPs com coeficientes racionais, o conjunto $\operatorname{conv}(X)$ é um poliedro e representa a melhor de todas as formulações de $X$. Além disso, temos que

$$
z_{I P}=\min \{c x ; x \in \operatorname{conv}(X)\} \text {. }
$$

Como o problema min $\{c x ; x \in \operatorname{conv}(X)\}$ é um LP, podemos resolver o problema usando um algoritmo existente na literatura, como o método Simplex.

Para alguns problemas é fácil encontrar uma formulação compacta que corresponde, ao mesmo tempo, ao envoltório convexo de soluções factíveis. É o caso do problema de transporte, do fluxo máximo, por exemplo. Estes problemas têm como característica comum que sua formulação é dada por uma matriz totalmente unimodular. Infelizmente isso não ocorre para a maioria dos COPs. Encontrar o conjunto de inequações que descrevem o $\operatorname{conv}(S)$ não é uma tarefa fácil. Além disso, muitas vezes o número de desigualdades, nesta formulação, cresce exponencialmente em relação ao número de variáveis e restrições.

Uma alternativa para esta dificuldade consiste em obter uma aproximação para o $\operatorname{conv}(S)$. Uma aproximação para o $\operatorname{conv}(S)$ pode ser construída de forma gradativa, acrescentando inequações à região de factibilidade do problema e que estas estejam próximas das que descrevem o $\operatorname{conv}(S)$. As inequações acrescentadas à região de factibilidade são denominadas de inequações válidas.

Definição 4. Uma inequação $\pi x \leq \pi_{0}$ é denominada de inequação válida para o poliedro $P \subseteq \mathbb{R}^{n}$ se $\pi x \leq \pi_{0}$ para todo $x \in P$.

Note que $\pi x \leq \pi_{0}$ é uma inequação válida, se e somente se, $P \subseteq\left\{x \in \mathbb{R}^{n}: \pi x \leq \pi_{0}\right\}$. As inequações válidas $\pi x \leq \pi_{0}$ e $\gamma x \leq \gamma_{0}$ são denominadas equivalentes se $\left(\pi, \pi_{0}\right)=\lambda\left(\gamma, \gamma_{0}\right)$, para algum $\lambda>0$. A inequação válida $\gamma x \leq \gamma_{0}$ domina ou é "mais forte" que $\pi x \leq \pi_{0}$ se existe $\mu>0$ tal que $\gamma \geq \mu \pi$ e $\gamma_{0} \leq \mu \pi_{0}$.

Definição 5. Seja $P \subseteq \mathbb{R}^{n}$ um poliedro. Um conjunto $F \subseteq P$ é denominado de face de $P$ se existe uma inequação válida $\pi x \leq \pi_{0}$ para $P$, tal que $F=\left\{x \in P: \pi x=\pi_{0}\right\}$ e dizemos que $F$ é induzida ou definida pela inequação $\pi x \leq \pi_{0}$. Além disso, se $F \neq P$ e $F \neq \emptyset$, então dizemos que F é uma face própria.

Observe que se $F$ é uma face de um poliedro $P$ definida pela inequação válida $\pi x \leq \pi_{0}$, então $F=\left\{x ; A x \leq b, \pi x \leq \pi_{0},-\pi x \leq-\pi_{0}\right\}$. Logo, $F$ também é um poliedro.

Como as inequações válidas são ferramentas para descrever o conv $(S)$, precisamos identificar quais as necessárias ou as mais importantes. Tais questões estão relacionadas com a dimensão de $\operatorname{conv}(S)$ e da face induzida por uma inequação válida.

Definição 6. O conjunto de pontos $x^{1}, x^{2}, \ldots, x^{k} \in \mathbb{R}^{n}$ é afim-independente se a solução para $\sum_{i=1}^{k} \alpha x^{i}=0$, em que $\alpha_{i} \in \mathbb{R} e \sum_{i=1}^{k} \alpha_{i}=0$, é obtida somente quando $\alpha_{i}=0, i=1, \ldots, k$. 
Definição 7. Um poliedro $P \subseteq \mathbb{R}^{n}$ é de dimensão $k$, denotado por $\operatorname{dim}(P)=k$, se o número máximo de vetores afim-independentes em $P$ é $k+1$.

Definição 8. Um poliedro $P \subseteq \mathbb{R}^{n}$ é de dimensão completa se $\operatorname{dim}(P)=n$.

Pode-se mostrar que, se $P \subseteq \mathbb{R}^{n}$ é um poliedro, então $\operatorname{dim}(P)=n-\operatorname{rank}\left(A^{=}, b^{=}\right)$, em que $\operatorname{rank}\left(A^{=}, b^{=}\right)$denota o posto da matriz $\left(A^{=}, b^{=}\right)$. Assim, o poliedro $P$ é de dimensão completa se não houver equações na representação de $P$.

Definição 9. Seja $F$ uma face própria de um poliedro $P \subseteq \mathbb{R}^{n}$. A face $F$ é denominada de faceta de $P$ se $\operatorname{dim}(F)=\operatorname{dim}(P)-1$.

Teorema 1. Se F é uma faceta de $P$, então existe alguma inequação $a^{k} x \leq b_{k}$, com $k \in M \leq$, que representa $F$.

Os próximos resultados estabelecem quais inequações são necessárias para descrever o poliedro $P$ e quais podem ser omitidas.

Teorema 2. Seja $P \subseteq \mathbb{R}^{n}$ um poliedro.

i) Para cada faceta $F$ de $P$, uma das inequações que representa $F$ é necessária na descrição de $P$.

ii) Toda inequação $a^{r} x \leq b_{r}$ para $r \in M \leq$ que define uma face do poliedro $P$ de dimensão menor que $\operatorname{dim}(P)-1$ é irrelevante na descrição de $P$.

Teorema 3. Seja $P \subseteq \mathbb{R}^{n}$ um poliedro.

i) Se $P$ é de dimensão completa, então o poliedro tem uma representação mínima e única (a menos de um escalar) composta por um conjunto de inequações lineares. Para cada faceta $F_{i}$ de $P$, existe uma inequação $a^{i} x \leq b_{i}$ que a define e o poliedro $P$ é descrito por $\left\{x \in \mathbb{R}^{n}: a^{i} x \leq b_{i}, i=1, \ldots, t\right\}$.

ii) Se $\operatorname{dim}(P)=n-k$, com $k>0$, uma descrição mínima de $P$ deve conter $k$ linhas linearmente dependentes de $\left(A^{=}, b^{=}\right)$. Além disso, para toda faceta $F_{i}$ de $P$ definida por $a_{i} x \leq b_{i}$, deve existir em $P$ uma desigualdade da forma $\left(\pi, \pi_{0}\right)=\lambda\left(a_{i}, b_{i}\right)+u\left(A^{=}, b^{=}\right)$.

Os resultados acima mostram que as facetas dão a melhor representação possível de um poliedro. É verdade, entretanto, que nem sempre é possível encontrar tal representação.

\subsection{Métodos exatos}

Na sequência, apresentamos três métodos exatos para resolver COP: branch and bound, planos de corte e branch and cut. 


\subsubsection{Algoritmo branch and bound}

O algoritmo branch and bound $(B \& B)$, proposto por Land e Doig (1960), é um método exato para resolver MILPs e IPs. Em muitos problemas, a enumeração completa e a avaliação de todas as soluções de um MILP/IP são tarefas inviáveis. Uma maneira de reduzir o espaço de soluções consiste em usar de forma inteligente os limitantes superior e inferior para descartar, quando possível, um subconjunto de soluções. Os subconjuntos descartados são definidos pela estratégia de dividir para conquistar e representam partes da árvore de decisão que não precisam ser exploradas, simplesmente por ter certeza que a solução ótima não estará nesta região.

O limitante inferior pode ser obtido pela relaxação linear do problema. Para isso, considere o $L P$ obtido quando a restrição de integralidade da variável $x_{j}$ do problema $P_{M I L P}$, $j \in \mathscr{Z}$, é substituída para valores reais. O problema resultante é definido por

$$
\begin{aligned}
\left(P_{\text {RMILP }}\right) \quad z_{\text {RMILP }}= & \text { Minimizar } \\
& \text { Sujeito a } \quad A x \leq b, \\
& x_{j} \in \mathbb{R}_{+}, \quad \forall j \in \mathscr{Z} \cup \mathscr{C} .
\end{aligned}
$$

O valor de $z_{R M I L P}$ representa um limitante inferior para o valor da solução ótima do problema $P_{M I L P}$. Além disso, o valor $\bar{z}$ de qualquer solução factível de $P_{M I L P}$ é um limitante superior para $z_{M I L P}$. Portanto, temos que $z_{R M I L P} \leq z_{M I L P} \leq \bar{z}$.

No algoritmo $B \& B$, a estratégia de dividir para conquistar consiste em dividir a região de factibilidade do MILP em sub-regiões e, em seguida, cada sub-região é explorada de forma recursiva.

Tal processo pode ser representado através de uma árvore $B \& B$ ou árvore de decisão. Cada nó da árvore de decisão corresponde a um subproblema. O nó raiz da árvore é representado pela relaxação do $P_{M I L P}$. Se a solução de $P_{R M I L P}$ satisfaz as restrições de integralidade para as variáveis $x_{j}, j \in \mathscr{Z}$, então a solução do MILP foi encontrada. Caso contrário, o valor de $z_{R M I L P}$ torna-se um limitante inferior inicial para $z_{M I L P}$ e o nó raiz é particionado em dois ramos. Cada ramo representa um novo subproblema. Essa etapa é denominada de ramificação (branching) e consiste em adicionar restrições lineares ao problema de tal forma que a solução ótima de $P_{R M I L P}$ seja eliminada, a região de factibilidade dos dois subproblemas seja mutuamente exclusiva e a sua união inclua todas as soluções factíveis de seu predecessor. Por exemplo, em um BP, um subproblema é criado fixando uma variável $x_{j}, j \in \mathscr{Z}$, igual a 0 e outro subproblema quando $x_{j}$ for igual a 1 . Na sequência é escolhido um novo nó na árvore de decisão. Dessa forma, a estrutura da árvore de decisão mantém o controle de como os subproblemas são gerados e resolvidos.

De forma geral, dado um nó $i$ na árvore de decisão, sejam $P_{R M I L P}^{i}$ a relaxação do subproblema associado a $i$ e $z_{R M I L P}^{i}$ a sua solução ótima. Além disso, considere $z_{I P}$ como sendo o valor da melhor solução factível encontrada até o momento. A partir do valor de $z_{R M I L P}^{i}$, é possível verificar se é necessário ou não realizar uma ramificação neste nó. Se a solução ótima satisfaz as restrições de integralidade, então a exploração do nó é finalizada e, quando for o caso, o valor de $z_{I P}$ é atualizado. Caso contrário, se $z_{R M I L P}^{i} \geq z_{I P}$, e como $z_{R M I L P}^{i}$ é um limitante inferior para o problema, então não será possível encontrar uma solução factível de valor menor que $z_{I P}$. Assim, o nó $i$ é podado, isto é, a exploração do nó é 
finalizada. O mesmo ocorre se o subproblema for infactível. Por fim, se $z_{R M I L P}^{i}<z_{I P}$, então é realizada a ramificação no nó $i$.

Um nó não explorado (não podado) na árvore de decisão é denominado de ativo. O algoritmo $B \& B$ é finalizado quando não houver nós ativos na árvore de decisão.

Duas das principais decisões do algoritmo $B \& B$ estão relacionadas com as estratégias utilizadas para a seleção do nó e da variável para a ramificação. A primeira delas consiste em escolher o nó ativo que será explorado na árvore de decisão. Existem duas possibilidades: regras a priori e regras adaptativas. As regras a priori determinam previamente a ordem em que a árvore de decisão será explorada. A regra a priori mais conhecida é a busca em profundidade com backtracking, em que, se um nó não é podado, então o próximo nó a ser explorado é um de seus filhos (last in, first out). Nas regras adaptativas, o nó é selecionado a partir das informações dos nós ativos. Um exemplo de critério utilizado baseia-se em selecionar o nó com melhor limitante inferior.

Se o nó selecionado não for podado, então a próxima etapa do algoritmo $B \& B$ compreende definir a variável que será utilizada na etapa de ramificação. Várias estratégias são utilizadas na literatura. Dependendo do problema, a ordem da escolha pode ser um parâmetro de entrada do algoritmo. Outras estratégias consistem, por exemplo, em selecionar a variável com a parte fracionária mais próxima de 0,5 (maximum integer infeasibility), ou selecionar a variável com a parte fracionária mais próxima de um número inteiro (minimum integer infeasibility). O trabalho de Achterberg, Koch e Martin (2005) descreve e analisa as principais estratégias de ramificação utilizadas na literatura e propõe uma nova abordagem denominada reliability branching.

As estratégias de ramificação citadas anteriormente levam em consideração que o método B\&B é aplicado em um MILP. Entretanto, a técnica B\&B pode ser utilizada em outros modelos, como por exemplo, em grafos. Nesta situação, a estratégia de ramificação pode ser estabelecida adotando uma direção para algumas das arestas (CARLIER; PINSON, 1989).

\subsubsection{Algoritmo de planos de corte}

O algoritmo de planos de corte foi proposto por Gomory (1958) no final da década de 50 como uma estratégia para resolver MILPs. Entretanto, os planos de corte de Gomory não foram eficientes em experimentos práticos, pois eram "fracos" e causavam instabilidade numérica.

Um algoritmo de planos de corte (PC) é um algoritmo iterativo que procura melhorar a relaxação de um MILP através do acréscimo de planos de corte e, consequentemente, aumentar o valor do limitante inferior (problema de minimização). Inicialmente, o algoritmo determina a solução ótima para o problema $P_{R M I L P}$. Se $P_{R M I L P}$ é infactível, então o mesmo ocorre com o problema original $P_{M I L P}$. Se o valor de cada variável $x_{j}, j \in \mathscr{Z}$, da solução ótima de $P_{R M I L P}$ é um número inteiro, então o algoritmo encontrou a solução ótima para $P_{M I L P}$. Caso contrário, determina-se um conjunto de inequações que são válidas para toda solução de $P_{M I L P}$, mas que são violadas pela solução da relaxação do problema e este conjunto é adicionado ao problema corrente. O algoritmo continua até que ou uma solução inteira é obtida, ou a relaxação linear corrente é infactível, ou não é possível encontrar mais planos de corte. Embora o algoritmo de PC esteja classificado como um método exato, o método terá este comportamento somente se as restrições de integralidade forem satisfeitas. Caso 
contrário, se o problema for factível, então o valor da função objetivo representa um limitante inferior para o problema.

Assim, espera-se, a cada iteração, que a relaxação do problema corrente esteja mais "justa", isto é, mais próxima do envoltório convexo da região de factibilidade do problema $P_{M I L P}$.

O termo planos de corte é utilizado para expressar as inequações válidas utilizadas para eliminar (cut-off) a solução ótima da relaxação do problema corrente. O processo de encontrar planos de corte é denominado de problema de separação e um algoritmo para resolvê-lo é denominado de algoritmo de separação. Na maioria das situações, é um desafio encontrar um algoritmo de separação eficiente para uma família de inequações válidas. Em alguns casos, este objetivo pode ser atingido com algoritmos exatos, mas em outros é necessário o uso de heurísticas. Embora não haja garantia de encontrar uma desigualdade violada, quando esta existir, heurísticas são utilizadas para acelerar a solução do problema de separação (POCHET; WOLSEY, 2006).

Um importante teorema devido a Grötschel, Lovász e Schrijver (1988) estabelece a equivalência entre o problema de separação e o de otimização. Antes de apresentar o referido teorema, vamos definir formalmente o problema de separação e otimização.

Definição 10. Problema da Separação: dado um poliedro racional $P \subseteq \mathbb{R}^{n}$ e vetor racional $y \in \mathbb{R}^{n}$, decidir se $y \in P$, ou caso contrário, determinar uma inequação $\pi x \leq \pi_{0}$ que é válida para todo $x \in P$, mas $\pi y>\pi_{0}$.

Definição 11. Problema de Otimização: dado um poliedro racional $P \subseteq \mathbb{R}^{n}$ e vetor racional $c \in \mathbb{R}^{n}$, determinar $x^{*} \in P$ que minimize $c x$, para todo $x \in P$, ou concluir que $P$ é ilimitado.

Teorema 4. (GRÖTSCHEL; LOVÁSZ; SCHRIJVER, 1988) Para qualquer classe de poliedros, existe um algoritmo polinomial para o problema de separação se, e somente se, existe um algoritmo também polinomial para o problema de otimização.

Existem classes de inequações válidas que podem ser aplicadas em qualquer MILP, independentemente de sua estrutura. Entretanto, tais inequações podem não ser uma ferramenta eficiente para um problema particular. Neste caso, novas inequações válidas podem ser obtidas explorando a estrutura do problema.

\subsubsection{Algoritmo branch and cut}

O método branch and cut $(B \& C)$ é um algoritmo exato que incorpora o método de PC dentro de um procedimento $B \& B$. De acordo com o trabalho de Macambira (2009), os primeiros trabalhos que combinam as duas técnicas são devidos a Miliotis (1976), Grötschel, Jünger e Reinelt (1984) e Padberg e Rinaldi (1991).

Conforme comentado anteriormente, o método $B \& B$ enumera todas as soluções factíveis de um problema através de uma estrutura de árvore de decisão. Assim, no método $B \& C$, para cada subproblema associado ao nó da árvore de decisão, é aplicado o método PC. Finalizado o método PC, se a solução ótima do subproblema obedece às restrições de integralidade ou se o limitante inferior do subproblema for maior que o valor de uma solução factível conhecida, então o respectivo nó é descartado e parte-se para a seleção de um novo 
nó. Agora, se não for possível encontrar uma inequação válida para o problema da separação, então aplica-se o procedimento de ramificação.

O benefício de incorporar o método PC em um nó da árvore de decisão está na possibilidade de melhorar o valor do limitante inferior do subproblema e portanto, quando possível, eliminar de forma mais rápida toda subárvore gerada pelo respectivo nó.

Além das estratégias inerentes do algoritmo $B \& B$, outras decisões provenientes do método de PC devem ser levadas em consideração durante a implementação do método. Essas decisões estão relacionadas com a eficiência do algoritmo de separação, à quantidade de inequações válidas e ao momento de acrescentá-las ao subproblema corrente. Na sequência, comentamos a respeito de cada uma dessas escolhas.

O processo de encontrar inequações violadas pode ser uma tarefa que necessite de elevado tempo de processamento. Uma abordagem utilizada com o intuito de reduzir o tempo computacional do algoritmo de separação compreende armazenar as inequações encontradas em um depósito (cut pool) e utilizá-las para busca futura. É importante que o depósito de inequações não seja grande, mas que contenha as inequações importantes. Para isso, o depósito de inequações pode ser equipado com um dispositivo de envelhecimento, em que inequações não violadas há algum tempo são eliminadas do depósito (FERREIRA; WAKABAYASHI, 1996; PFETSCH, 2008). Outra utilidade do depósito de inequações está relacionada com a etapa de ramificação do B\&C. Quando um nó na árvore de busca é selecionado, o depósito de inequações permite reconstruir o $L P$ que este nó dispunha na hora que foi criado. Veja os trabalhos de Padberg e Rinaldi (1991) e Ferreira e Wakabayashi (1996) para mais detalhes.

Quanto maior o acréscimo de inequações violadas, maior é o problema de programação linear a ser resolvido. Logo, mais tempo será necessário para resolvê-lo. Entretanto, embora adicionar mais inequações melhore a descrição do poliedro, o acréscimo de uma grande quantidade de inequações violadas não garante o aumento do limitante inferior. Assim, um problema a ser considerado é estabelecer a quantidade de inequações violadas que devem ser adicionadas ao LP corrente, se existir mais de uma disponível. Algumas estratégias podem ser adotadas:

- Escolha de inequações em classes diferentes. Espera-se com essa abordagem que a escolha de inequações de diversas classes seja mais efetiva do que a utilização de uma única classe (FERREIRA; WAKABAYASHI, 1996).

- Selecione as inequações mais violadas. Esta estratégia adiciona as $k$ inequações mais violadas, uma vez que elas podem ter a maior influência no processo de otimização (FERREIRA; WAKABAYASHI, 1996).

- Insere todas as inequações no nó raiz. As inequações válidas inseridas no nó raiz do B\&C também são válidas para todos os nós da árvore de decisão. Desta forma, esta estratégia permite que todas as inequações violadas acrescentadas ao sistema de inequações do nó raiz também sejam utilizadas no processamento dos demais nós. Conforme Pochet e Wolsey (2006), esse procedimento é denominado de cut and branching.

Outra decisão do algoritmo B\&C que também não tem uma resposta exata é estabelecer o momento em que devemos inserir os planos de corte na árvore de decisão e a quantidade de iterações do algoritmo de PC em cada nó da árvore. O manual de referência do resolvedor Gurobi recomenda que as inequações violadas devem ser adicionadas com moderação, uma 
vez que aumentam o tamanho do modelo de programação linear do atual nó e de todos os seus descendentes.

\subsection{Heurísticas}

Heurísticas são métodos que procuram encontrar boas soluções para um determinado problema de forma rápida. Entretanto, ao contrário dos métodos exatos, não há garantia para a qualidade da solução.

\subsubsection{Metaheurística}

Uma classe especial de heurísticas que tem recebido atenção é a metaheurística. As metaheurísticas são técnicas que buscam combinar métodos heurísticos em um arcabouço de mais alto nível permitindo explorar de forma eficiente o espaço de busca de soluções (BLUM; ROLI, 2003). O termo metaheurística foi introduzido no trabalho de Glover (1986), em que o método de Busca Tabu é visto como uma heurística sobreposta a outra heurística. Observamos que não há uma definição comum para o termo metaheurística. Alguns autores, como por exemplo Cunha et al. (2012), definem a metaheurística como um conjunto de conceitos que pode ser utilizado para definir métodos heurísticos aplicáveis a um extenso conjunto de diferentes problemas, enquanto outros, como Osman e Laporte (1996) e Vose (1999), a definem como procedimentos que guiam outras heurísticas, direcionando seus movimentos para explorar, de forma mais eficiente e eficaz, diferentes regiões do espaço de soluções além do ótimo local. Algumas metaheurísticas bem conhecidas são: busca tabu, simulated annealing, busca local iterada, greedy randomized adaptive search procedure (GRASP), variable neighborhood search, e algoritmos bio-inspirados tais como algoritmo genético, estratégias evolutivas e colônia de formigas.

\subsubsection{Busca Local}

A estratégia de busca local (LENSTRA, 2003) emprega a ideia de que uma solução factível para um POC pode ser melhorada através de pequenas mudanças. Esta estratégia explora o espaço de soluções de uma solução factível, indo de uma solução para outra, desde que esta esteja em sua vizinhança (CUNHA et al., 2012). Assim, o algoritmo procura encontrar melhores soluções na vizinhança de uma solução factível.

Considere o problema de minimização $\min \left\{f(S) \mid S \in \sum\right\}$ em que $f$ é a função objetivo e $\sum$ o espaço de busca, isto é, o conjunto de soluções factíveis para o problema. Uma função de vizinhança é um mapeamento $\mathcal{N}: \sum \rightarrow 2^{\sum}$ que define para cada solução $S \in \sum$, um subconjunto $\mathcal{N}(S)$ de $\sum$, chamado de vizinhança. Assim, cada elemento em $\mathscr{N}(S)$ é um vizinho de $S$. De um modo geral, um vizinho $S^{\prime} \in \mathcal{N}(S)$ de uma solução $S$ é uma solução obtida através de um movimento, definido a priori, a partir da solução corrente $S$. Um algoritmo de busca local inicia a partir de solução inicial e inspecionando a sua vizinhança, procura encontrar, de forma iterativa, melhores soluções. Uma maneira de determinar o melhor vizinho consiste em analisar o valor de $f\left(S^{\prime}\right)$ para cada $S^{\prime} \in \mathcal{N}(S)$. Dessa forma, a próxima solução de referência $S$ é a que produz o menor de valor para a função objetivo. O procedimento continua até que nenhum vizinho $S^{\prime} \in \mathcal{N}(S)$ com $f\left(S^{\prime}\right)<f(S)$ seja encontrado, ou até que algum outro critério de parada tenha sido satisfeito. 
Devido ao fato do algoritmo aceitar somente soluções $S^{\prime}$ que melhoram a solução atual $S$, não é possível de escapar de mínimos locais. Em muitas situações, o valor de $\min \left\{f(S) \mid S \in \sum\right\}$ é menor que o valor produzido pelo mínimo local. Metaheurísticas podem ser utilizadas para evitar esse problema. A busca local é uma estratégia utilizada em várias metaheurísticas, tais como busca tabu, simulated annealing e busca local iterada. 



\section{Capítulo 2 \\ O problema de job-shop flexível}

Neste capítulo, apresentamos formalmente o problema que é objeto de estudo nesta tese: o job-shop flexível. O job-shop flexível pertence à categoria de problemas de scheduling ou programação de tarefas. Dentre os diversos problemas desta categoria, o job-shop flexível é uma generalização do clássico problema de job-shop.

Inicialmente apresentamos as características que diferem os diversos problemas de programação de tarefas. Na sequência, destacamos o problema de job-shop e job-shop flexível. Além disso, como um dos objetivos desta tese consiste em utilizar um método B\&C para a resolução do job-shop flexível, também revisamos os trabalhos da literatura que utilizam MILPs para a resolução do problema.

\subsection{O problema de programação de tarefas}

O problema de programação de tarefas se refere a alocar recursos a tarefas, respeitando as restrições impostas e com o objetivo de otimizar uma ou uma combinação de medidas de desempenho (LEUNG, 2004). Dessa forma, busca-se a melhor forma de processar as tarefas através da definição do instante de início e término do processamento de cada tarefa. Dependendo do contexto, os recursos e tarefas podem ter diferentes significados. Os recursos podem representar as máquinas em uma linha de montagem, memória ou dispositivos de entrada e saída em um computador, pistas de um aeroporto e outros. As tarefas, objeto principal da programação, podem representar as várias operações em um processo de fabricação, execução de um programa de computador, aterrissagens e decolagens em um aeroporto e outros.

Segundo Leung (2004), o estudo dos problemas de programação de tarefas teve início na década de 1950 a partir de várias atividades do setor industrial. Os problemas estudados naquela época eram relativamente simples e foram desenvolvidos vários algoritmos que permitiam obter a solução ótima. Destacam-se, por exemplo, os trabalhos de Johnson (1954), Jackson (1955) e Jackson (1956).

No problema de programação em um ambiente de manufatura e produção, máquinas são usadas para processar um número de tarefas (jobs) com o intuito de produzir produtos. Para que uma tarefa seja finalizada, ela precisa ser processada por uma ou mais máquinas e cada etapa de processamento é denominada de operação.

Na língua portuguesa, observamos em algumas situações que o termo scheduling é traduzido como sequenciamento de tarefas. Conforme Pinedo (2012) e Baker e Trietsch (2013), 
sequenciamento corresponde à ordem em que as tarefas são processadas em um conjunto de máquinas e scheduling indica o processo de gerar o sequenciamento. Dessa maneira, o termo scheduling se refere, além da definição da sequência de processamento das tarefas, a determinar, por exemplo, o início e o término de processamento de cada operação. Assim, além de obter as informações relacionadas às tarefas, é possível estabelecer em quais intervalos de tempo as máquinas estão disponíveis ou alocadas durante o período de execução de todas as tarefas.

Ainda no ambiente de manufatura e produção, há uma grande variedade de problemas e configurações. Diversos tipos de classificações têm sido propostos, como por exemplo a categorização de Lenstra (1976), Lenstra e Kan (1978) e Graham et al. (1979). De maneira geral, os problemas de scheduling são classificados conforme o número e a disposição das máquinas, as restrições de precedência das operações em cada tarefa e o tipo da função objetivo. Para descrever as diversas configurações, utilizamos como referência o livro "Scheduling: Theory, Algorithms, and Systems" de Michel L. Pinedo (PINEDO, 2012).

Em relação às máquinas, as possíveis configurações são:

- Programação em uma única máquina: há uma única máquina no ambiente de produção. Este problema surge como um caso especial de outros problemas e seu estudo permite a compreensão e desenvolvimento de técnicas em ambientes mais complexos.

- Máquinas idênticas e paralelas: ambiente com $m$ máquinas idênticas e em paralelo no sistema e cada tarefa pode ser processada por qualquer máquina.

- Máquinas uniformes e paralelas: ambiente com $m$ máquinas em paralelo com diferentes velocidades de processamento. A velocidade de processamento não depende da tarefa selecionada.

- Máquinas uniformes e paralelas: ambiente com $m$ máquinas em paralelo com diferentes velocidades de processamento. A velocidade de processamento depende da máquina selecionada e da tarefa.

As diferentes formas de precedência entre as operações são descritas pelos problemas da família shop. De forma geral, nos problemas da categoria shop há um conjunto com $n$ tarefas, $m$ máquinas e cada tarefa é composta por um conjunto de operações. Duas operações de uma mesma tarefa não podem ser processadas de forma simultânea e cada máquina pode processar uma única operação em cada instante. Os problemas nesta categoria são:

- Open-shop: cada tarefa necessita ser processada por cada uma das $m$ máquinas, mas não há um fluxo definido para as operações de cada tarefa.

- Flow-shop: as $m$ máquinas estão em série e cada tarefa tem que ser processada em cada uma das máquinas. Todas as tarefas possuem o mesmo fluxo de processamento, isto é, as tarefas são processadas na máquina 1, na máquina 2 e assim por diante.

- Job-shop: cada tarefa tem a sua própria ordem de processamento.

A maioria das funções objetivo consiste em minimizar uma medida de desempenho. As principais medidas de desempenho estão em função do instante de término de conclusão das tarefas. Na sequência, apresentamos algumas das principais medidas de desempenho: 
- Makespan: representa o instante de término de processamento da última tarefa a deixar o sistema. Este critério foi o primeiro tipo de função objetivo aplicado pelos pesquisadores no início da década de 1950 (JAIN; MEERAN, 1999) e pode ser facilmente modelado.

- Adiantamento máximo (maximum lateness): dependendo das restrições de mercado, pode ser imposto um limite de tempo para a conclusão de cada tarefa (due date). O adiantamento de uma tarefa é definido pela diferença entre o seu instante de término e o limite de tempo para a conclusão. Se a diferença for negativa, então a tarefa está adiantada, caso contrário, a tarefa está atrasada. Esta medida de desempenho é apropriada quando há uma recompensa positiva para concluir uma tarefa de forma mais rápida; quanto mais cedo uma tarefa é concluída, maior a sua recompensa.

- Atraso total (total tardiness): diferença positiva entre o instante de término e o limite de tempo para a conclusão de todas as tarefas. Esta medida é apropriada quando não há recompensa em adiantar uma tarefa e apenas penalidades relacionadas ao atraso das tarefas.

- Soma ponderada do atraso total: para cada tarefa, há um peso relacionado ao seu atraso e é definido como a soma ponderada do atraso de cada tarefa. Esta medida é utilizada quando algumas tarefas são mais importantes do que outras.

Todas as funções de desempenho citadas anteriormente são denominadas de regulares. De acordo com Pinedo (2012), uma medida de desempenho é regular se for não-decrescente e expressa em função do instante de término de cada tarefa. Dessa forma, tais funções serão otimizadas iniciando o mais breve possível o processamento das operações.

Funções não regulares também são estudadas na literatura. Por exemplo, dentro da filosofia Just in Time das indústrias, os produtos devem ser finalizados o mais próximo possível do instante de início de sua utilização, dado que a antecipação de tarefas resulta em custos de estoque, armazenagem, deterioração, gasto com seguro, dentre outros. A medida de desempenho denominada earliness é definida, para cada tarefa, como o máximo entre o valor zero e diferença do limite de tempo para a conclusão e o seu instante de término. Como o earliness é não-crescente em relação ao instante de término da referida tarefa, a soma do earliness de todas as tarefas não é uma medida regular. Outra medida não regular bastante utilizada no contexto Just in Time é a soma das penalizações por adiantamento (earliness) e por atraso de todas as tarefas.

Na sequência deste capítulo, abordamos com mais detalhes o problema de job-shop e a sua versão generalizada, denominada de job-shop flexível.

\subsection{Job-shop}

O problema de job-shop (JS) consiste no planejamento de um conjunto de tarefas que devem ser executadas em um conjunto de máquinas. Para cada tarefa, é definida uma sequência de operações que devem ser processadas, cada uma delas em uma máquina específica. Não existem restrições de precedência entre as operações de tarefas distintas. Cada máquina executa uma única operação em cada instante e o seu processamento não pode ser interrompido após o seu início. Uma vez que a sequência de máquinas de cada tarefa é fixa, 
o problema a ser resolvido é dado pela pela distribuição das tarefas entre as máquinas de forma a otimizar uma função de desempenho.

O cálculo do makespan mínimo para o JS é um problema clássico de otimização combinatória que tem recebido considerável atenção na literatura e em problemas práticos (JAIN; MEERAN, 1999). Como veremos na sequência desta seção, além de ser um critério de fácil formulação, ele tem uma interpretação interessante na formulação do problema através de um grafo.

De acordo com Carlier e Pinson (1989), o desenvolvimento de métodos de resolução para o JS começa com o livro Industrial Scheduling, de Muth e Thompson (MUTH; THOMPSON, 1963), no qual foram propostos três instâncias com 10 máquinas e 10 tarefas. Dois desses problemas foram resolvidos na década de 70 por McMahon e Florian (1975) e Carlier (1978) e o último foi resolvido somente em 1986 por Carlier e Pinson (1989).

Embora existam algoritmos que resolvem o JS de forma ótima, como por exemplo o método B\&B proposto por Carlier e Pinson (1989), devido à sua natureza combinatória, o tempo de processamento cresce exponencialmente em função dos dados de entrada. Para um problema com $m$ máquinas e $n$ tarefas, em que qualquer operação de uma determinada tarefa é executada em uma máquina diferente das demais operações, o número máximo de sequenciamentos possíveis é igual a $n !^{m}$.

O JS, além de ser um problema NP-difícil (GAREY; JOHNSON; SETHI, 1976), é um dos mais difíceis de ser tratado nessa classe (LAWLER et al., 1993). Para alguns casos particulares, a solução do JS pode ser obtida em tempo polinomial, tais como: JS composto por duas tarefas (BRUCKER, 1988), o problema de Flow-Shop com duas máquinas (JOHNSON, 1954), o JS com duas máquinas em que cada tarefa possui no máximo duas operações (JACKSON, 1956), o JS com duas máquinas e com tempo de processamento unitário (HEFETZ; ADIRI, 1982) e o JS com duas máquinas e com um número fixo de tarefas (BRUCKER; JURISCH; SIEVERS, 1994).

\subsubsection{Descrição do job-shop e formulação matemática}

Para descrever o JS, considere $T$ o conjunto de $n$ tarefas e $M$ o conjunto de $m$ máquinas. Cada tarefa $i \in T$ é composta por uma sequência de $n_{i}$ operações:

$$
O_{i 1} \rightarrow O_{i 2} \rightarrow \cdots \rightarrow O_{i n_{i}}
$$

Cada operação $O_{i j}$ requer o uso exclusivo de uma única máquina já definida $m_{i j} \in M$ por um período de tempo $p_{i j}>0$. Supomos que $m_{i j} \neq m_{i j+1}$ pois, caso contrário, as operações $O_{i j}$ e $O_{i j+1}$ podem ser unidas e representadas por uma única operação. $\mathrm{O}$ conjunto $\mathcal{O}_{k}$ representa as operações que são processadas pela máquina $k \in M$. O objetivo do problema consiste em determinar o início do processamento de cada operação, tal que o maior tempo de conclusão dentre todas as operações seja o menor possível, isto é, minimizar o makespan.

Para exemplificar, considere uma instância do JS composta por três tarefas e cada tarefa constituída por três operações. O tempo de processamento e a máquina designada para processar cada operação estão descritos na Tabela 1.

O processamento das operações nas máquinas pode ser representado pelo diagrama de Gantt. Esse tipo de diagrama é uma ferramenta conveniente para visualizar uma solução para o JS, pois descreve o sequenciamento das tarefas e a ocupação das máquinas pelas operações em função do tempo. No diagrama, o eixo horizontal representa o tempo e o eixo vertical as 
Tabela 1 - Exemplo de uma instância do JS com três tarefas e três operações.

\begin{tabular}{cccc}
\hline Tarefa & Operação & Máquina & Duração (min) \\
\hline \multirow{2}{*}{1} & $O_{11}$ & 2 & 2 \\
& $O_{12}$ & 3 & 3 \\
& $O_{13}$ & 1 & 2 \\
\hline \multirow{3}{*}{2} & $O_{21}$ & 3 & 4 \\
& $O_{22}$ & 1 & 5 \\
& $O_{23}$ & 2 & 2 \\
\hline \multirow{3}{*}{3} & $O_{31}$ & 1 & 1 \\
& $O_{32}$ & 3 & 4 \\
& $O_{33}$ & 2 & 2 \\
\hline
\end{tabular}

máquinas. Cada retângulo representa o processamento de uma operação. O comprimento do retângulo é definido pelo tempo de processamento da operação. As figuras 1 e 2 apresentam duas soluções para a instância do JS descrito pela Tabela 1.

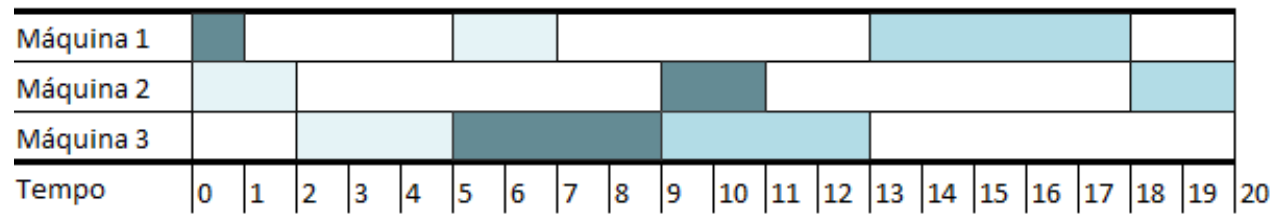

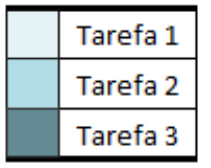

Figura 1 - Diagrama de Gantt de uma solução factível para o JS - Solução 1.

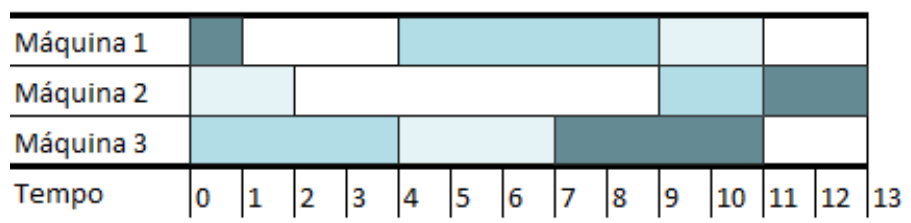

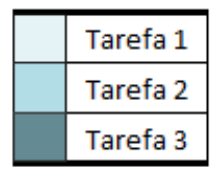

Figura 2 - Diagrama de Gantt de uma solução factível para o JS - Solução 2.

No sequenciamento descrito pela solução 1 (Figura 1), inicialmente as operações da tarefa 1 são atribuídas para cada uma das máquinas e, na sequência, de acordo com a ociosidade das máquinas, são designadas as operações das tarefas 3 e 2 . Os espaços em branco nos diagramas das soluções 1 e 2 indicam que a máquina está parada. Observe que a solução 2 é melhor que a solução 1 devido ao fato de encontrarmos menos tempo ocioso nas máquinas. Ao diminuir a ociosidade entre as máquinas, o tempo final de execução das tarefas será menor. O makespan da solução 2 é igual a 13 minutos, enquanto o da solução 1 é 20 minutos.

O JS também pode ser descrito por um MILP. Para descrever o modelo, considere a variável de decisão $s_{i}$ como sendo o início de processamento da operação $i$ na máquina $m_{i} \in M$. Para cada par de operações $[i, j] \in D$, a variável binária $y_{i j}$ é utilizada para expressar a ordem de processamento das operações $i$ e $j$. Então, $y_{i j}$ recebe o valor 1 se a operação $i$ é sequenciada antes da operação $j$, e zero caso contrário. Portanto, o JS pode ser formulado por 


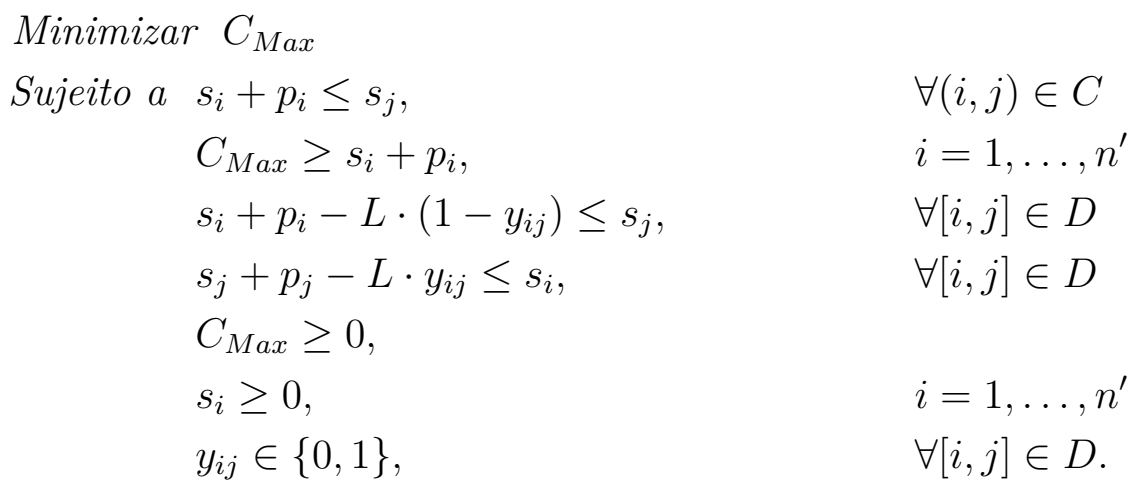

A função objetivo é expressa em (2.1) e o valor do makespan está definido pela restrição do tipo (2.3). A restrição do tipo (2.2) estabelece a ordem de processamento das operações em cada tarefa. As restrições (2.4) e (2.5) representam as restrições disjuntivas para cada máquina e garantem que cada máquina processe uma única operação ao mesmo tempo. A constante $L$ representa um limitante superior da duração total do sequenciamento. O valor de $L$ pode ser adotado como sendo a soma do tempo de processamento de cada operação. As restrições (2.5), (2.6) e (2.7) indicam as variáveis de decisão.

Conforme a maneira em que a variável binária descreve a ordem da execução das operações em cada máquina, outras formulações podem ser encontradas na literatura. O trabalho de Gomez Morales (2012) faz uma análise comparativa entre cinco formulações para o JS e descreve as suas vantagens e desvantagens.

Uma segunda maneira de descrever o JS é através de um grafo, denominado grafo disjuntivo. Essa modelagem foi proposta por Roy e Sussmann (1964). Os principais métodos baseados na técnica B\&B utilizam a modelagem por grafo disjuntivo para representar sequenciamentos para o JS. Um grafo disjuntivo $G=(V, C \cup D)$ é um grafo misto composto por um conjunto $\mathrm{V}$ de vértices, um conjunto $C$ de arcos direcionados, denominados de arcos conjuntivos, e um conjunto $D$ de arcos não-direcionados (arcos disjuntivos). Em correspondência com o JS, $V$ representa as operações de todas as tarefas, além das operações fictícias 0 e $F$. As operações 0 e $F$ denotam, respectivamente, o início e o término do processamento de cada tarefa. Para descrever o grafo disjuntivo, vamos simplificar a notação identificando as operações $O_{i j}$ pelos números $1, \ldots, n^{\prime}$, em que $n^{\prime}$ é o número total de operações, isto é, $n^{\prime}=\sum_{j=1}^{n} n_{j}$. Usaremos novamente o índice $i$ para as operações $i=1, \ldots, n^{\prime}$. Assim sendo, o tempo de processamento da operação será denotado por $p_{i}$. Cada vértice $i$ de $G$ tem um peso que é igual a $p_{i}$. Os valores de $p_{0}$ e $p_{F}$ são nulos. O conjunto $C$ representa a ordem de execução das operações em cada tarefa e o conjunto $D$ se refere às diferentes ordens em que as operações numa mesma máquina podem ser escalonadas. Se $i$ e $j$ são duas operações associadas a uma mesma tarefa, tal que $i$ precede de forma imediata $j$, então associamos ao grafo o arco conjuntivo $(i, j)$ (a notação $(i, j)$ indica o arco direcionado de $i$ para $j$ ). Para cada par de operações $i$ e $j(i \neq j)$ tal que $m_{i}=m_{j}$, associamos o arco disjuntivo denotado por $[i, j]$. A Figura 3 ilustra o grafo disjuntivo do JS descrito pela Tabela 1. Os arcos pontilhados representam os arcos disjuntivos e os contínuos denotam os arcos conjuntivos. Em cada vértice, o número interno se refere ao índice da operação e o externo ao tempo de processamento.

O problema de determinar um sequenciamento para o JS consiste em definir a ordem das operações para cada máquina. Na formulação por grafo disjuntivo, isto equivale a atribuir uma direção para cada arco disjuntivo $[i, j]$ tal que o grafo correspondente seja acíclico. 


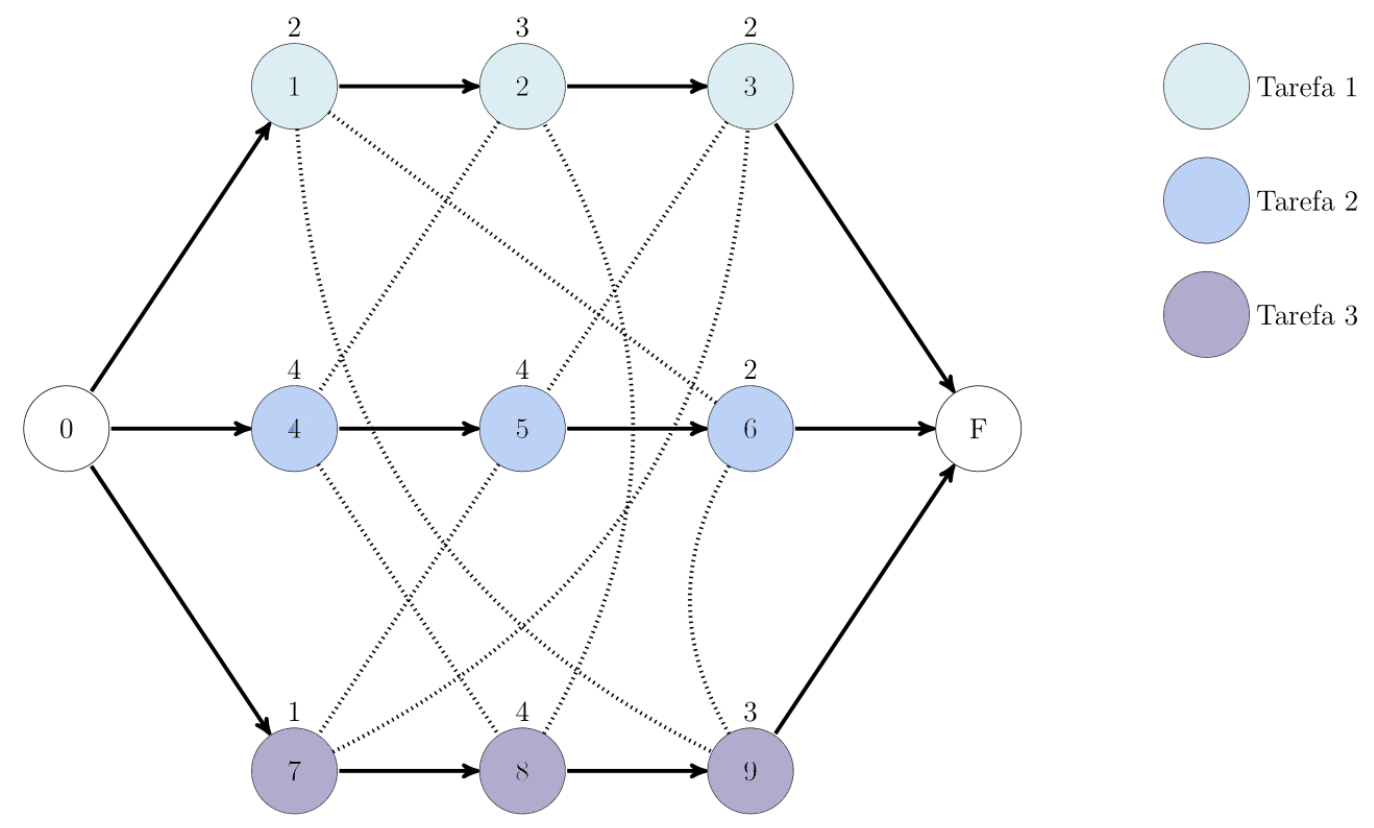

Figura 3 - Grafo disjuntivo para o JS descrito pela Tabela 1.

Uma seleção $S$ é um conjunto de arcos disjuntivos orientados, isto é, se $(i, j) \in S$ então $(j, i) \notin S$, tal que $[i, j] \in D$. Uma seleção é completa se todas as disjunções de $D$ são selecionadas. Dada uma seleção $S$, se o grafo $G_{S}=(V, C \cup S)$ é acíclico, então $S$ é denominada de consistente. Assim, um sequenciamento corresponde a uma seleção completa e consistente. Na Figura 4, cada grafo representa a seleção completa associada às soluções 1 e 2 .

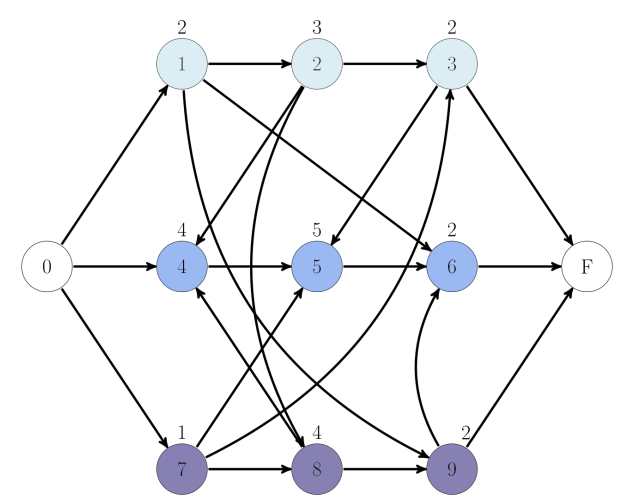

(a) Sequenciamento da Solução 1.

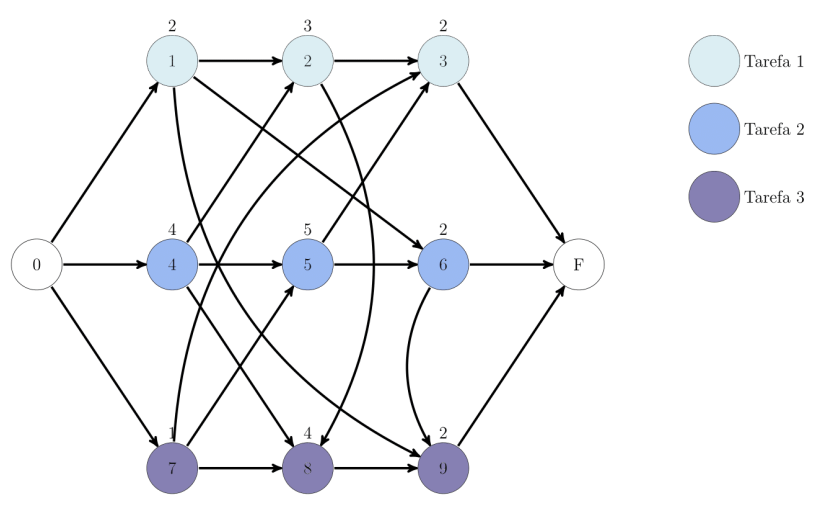

(b) Sequenciamento da Solução 2.

Figura 4 - Representação da seleção completa e consistente das soluções 1 e 2.

O comprimento de uma sequência de operações é definido pela soma de seus tempos de processamento. O caminho máximo entre duas operações $i$ e $j$ é representado pelo caminho de maior comprimento e seu valor é denotado por $L(i, j)$. Para uma determinada seleção completa e consistente $S$, o comprimento do caminho máximo entre os nós 0 e $F$ no grafo $G_{S}$ é, frequentemente, denominado de caminho crítico e denotado por $L(S)$. Observe que o valor $L(S)$ representa o tempo mínimo necessário para finalizar todas as tarefas, isto é, o makespan. 
Cada operação no caminho crítico é imediatamente seguida pela próxima operação executada na mesma máquina ou pela próxima operação da mesma tarefa. Além disso, para todo par de operações consecutivas $i$ e $j$ do caminho crítico, temos que $L(i, j)=p_{i}$ (NAJID; DAUZERE-PÉRÈS; ZAIDAT, 2002). Dessa forma, o valor de $L(S)$ é definido pela soma do tempo de processamento das operações que compõem o caminho crítico.

A Figura 5 indica o caminho crítico para as soluções 1 e 2. Na solução 1, o caminho crítico é obtido pela sequência de vértices $O, 1,2,8,4,5,6$ e $F$. O valor desse caminho é igual a 20. Para a solução 2, a sequência do caminho crítico é definida pelos vértices $O, 4,2$, 8, 9 e $F$, cujo comprimento é igual a 13. Observe que o caminho crítico para a solução 2 não é único, pois a sequência $O, 4,5,6,9$ e $F$ também tem comprimento igual a 13 .

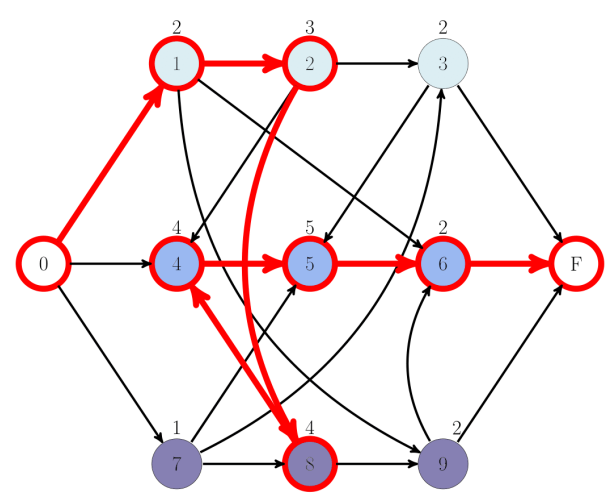

(a) Caminho crítico da solução 1.

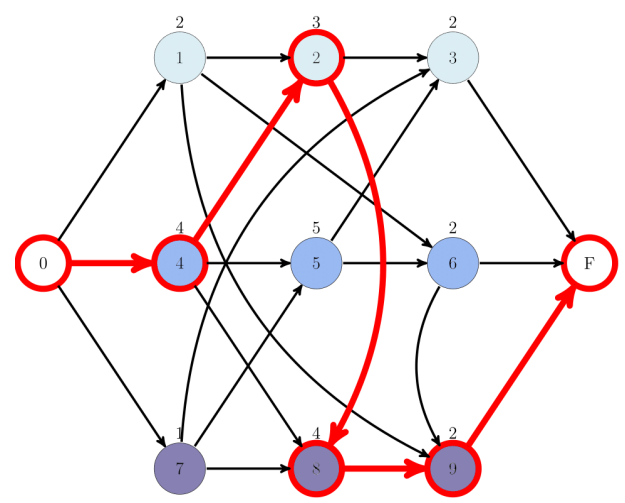

(b) Caminho crítico da solução 2.

Figura 5 - Representação do caminho crítico para as soluções 1 e 2.

Logo, o problema de JS consiste em determinar, entre todas as seleções completas e consistentes, qual possui o menor valor possível para o caminho crítico entre os nós 0 e $F$.

Dada uma seleção $S$ completa e consistente, algumas arestas consideradas redundantes podem ser eliminadas do grafo $G_{S}$. Se $i, j$ e $k$ são operações executadas em uma mesma máquina e $(i, j)$ e $(j, k) \in S$, então devemos ter $(i, k) \in S$. A aresta $(i, k) \in S$ pode ser retirada do grafo $G_{S}$, uma vez que $L(i, j)+L(j, k) \geq L(i, k)$. A Figura 6 apresenta o grafo associado às soluções 1 e 2, em que arcos redundantes foram removidos. Na Figura 6-(a), foram retirados os arcos redundantes $(1,6),(2,4)$ e $(7,5)$, enquanto em 6 -(b) foram removidos os $\operatorname{arcos}(1,9),(4,8)$ e $(7,3)$. Observe que no grafo resultante, para as operações executadas em uma mesma máquina, temos uma única aresta chegando e saindo da operação, desde que esta não seja nem a primeira e nem a última a ser executada na máquina. A redução de arcos no grafo tem um grande impacto em qualquer algoritmo que explora a ideia do grafo disjuntivo (BRANDIMARTE, 1993). Em teoria dos grafos, a eliminação de todos os arcos redundantes equivale a obter a redução transitiva (transitive reduction) de um digrafo direcionado. $\mathrm{O}$ trabalho de Aho, Garey e Ullman (1972) apresenta uma descrição completa do problema da redução transitiva.

Os principais trabalhos que utilizam a abordagem de grafo disjuntivo em um método B\&B para resolver o JS são devidos a Carlier e Pinson (1989), Applegate e Cook (1991) e Brucker, Jurisch e Sievers (1994). O algoritmo B\&B é retratado por uma árvore de enumeração. O nó raiz representa o grafo disjuntivo em que nenhuma direção é atribuída para os arcos disjuntivos. Logo, o nó raiz se refere a todas as soluções factíveis para o problema. Os nós sucessores são gerados atribuindo uma direção para os arcos disjuntivos. A exploração de um nó é finalizada se o respectivo nó representa uma solução factível ou se não for possível 


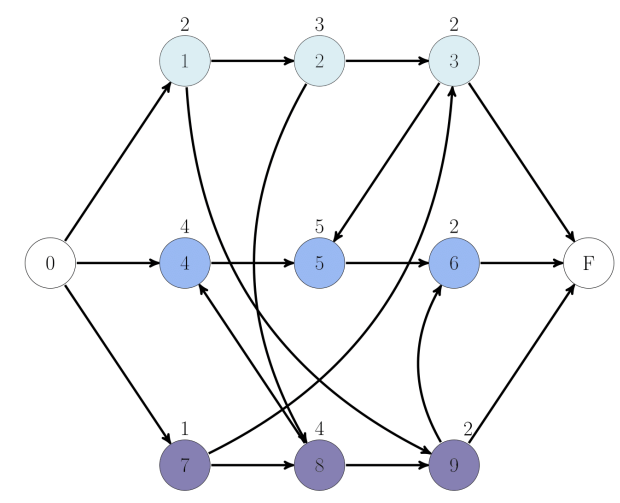

(a) Sequenciamento da solução 1.

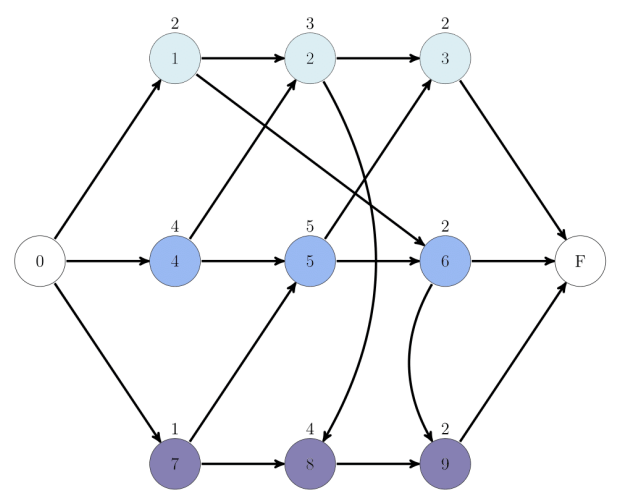

(b) Sequenciamento da solução 2.

Figura 6 - Representação das soluções 1 e 2 sem arcos redundantes.

encontrar uma solução ótima. Na sequência, apresentamos as principais contribuições em cada proposta.

A estratégia de ramificação do método B\&B consiste em atribuir uma direção para os arcos disjuntivos do grafo. Visando diminuir o número de possibilidades de escolhas de arcos disjuntivos para a ramificação, Carlier e Pinson (1989) desenvolveram o conceito de seleção imediata. Este conceito permite previamente fixar alguns arcos disjuntivos. Na abordagem proposta, as disjunções são fixas através de input e output de uma clique. Os autores definem uma clique $E$ como sendo um conjunto de operações executadas por uma mesma máquina com pelo menos dois elementos e que quaisquer pares de elementos em $E$ representam uma disjunção não fixa. Uma operação $e \in E$ é denominada input da clique $E$ se $e$ for sequenciada antes de todas as operações de $E \backslash\{e\}$, e $s \in E$ é um output de $E$ se $s$ for sequenciada após todas as operações de $E \backslash\{s\}$. Se a operação $e$ é um input de $E$, então o arco disjuntivo $[e, j]$ deve ser fixado na direção $e \rightarrow j$, para todo $j \in E \backslash\{e\}$. O mesmo ocorre com um output de uma clique. Se $s$ é um output de $E$, então o arco disjuntivo $[j, s]$ deve ser fixado na direção $j \rightarrow s$, para todo $j \in E \backslash\{s\}$. Carlier e Pinson apresentam um conjunto de propriedades que fornecem condições suficientes para que um vértice seja um input ou output de uma clique e, consequentemente, fixar os arcos disjuntivos relacionados a esses elementos. Posteriormente, em Carlier e Pinson (1990), é apresentado um procedimento mais eficiente de fixar os arcos disjuntivos através da atualização do head e tail de uma operação. A definição de head e tail de uma operação será apresentada no próximo capítulo (seção 3.1).

Para obter bons limitantes para o JS, Applegate e Cook (1991) desenvolveram um algoritmo de PC composto por 8 classes de inequações válidas: basic cuts, two-job cuts, clique cuts, two-job e two-machine cuts, triangle cuts, basic cuts plus epsilon, half cuts e late job cuts. Algumas destas classes originam uma nova classe, denominada de reversa, ao considerar a ordem inversa do sequenciamento das operações. Além disso, no algoritmo de PC foram utilizadas duas formulações para o JS, uma baseada em programação disjuntiva e outra em MILP. Embora o valor limitante inferior produzido pelo algoritmo PC tenha sido promissor, o tempo de processamento foi decepcionante. À vista disso, para o cálculo de limitante inferior no algoritmo B\&B, os autores utilizaram o algoritmo preemptivo de Jackson na programação das operações que são executadas em uma mesma máquina. Para mais detalhes sobre o algoritmo preemptivo de Jackson, remetemos ao trabalho de Carlier (1982).

O método B\&B proposto por Brucker, Jurisch e Sievers (1994) utiliza o conceito de blocos para a estratégia de ramificação. Esse conceito foi inicialmente utilizado por Grabowski, 
Nowicki e Zdrzałka (1986) como estratégia de ramificação para o problema do programação de tarefas em uma única máquina com head e tail. Uma sequência de operações $u_{1}, u_{2}, \ldots, u_{k}$ em um caminho crítico é denominada de bloco se a sequência contém pelo menos duas operações e representa o número máximo de operações executadas em uma mesma máquina. A Figura 7 apresenta a sequência de operações no caminho crítico das soluções 1 e 2 . Na solução 1 (Figura 7-(a)), temos um único bloco formado pela sequência de operações 2-8-4 na máquina 3. As demais operações do caminho crítico não formam um bloco pois não há ao menos duas consecutivas operações executadas em uma mesma máquina. Para a solução 2, temos o bloco formado pelas operações 4-2-8 (Figura 7-(b)).

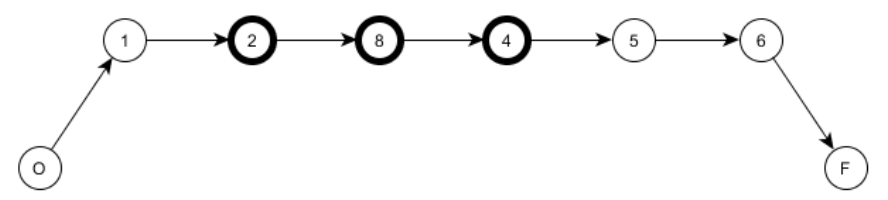

(a) Bloco e caminho crítico da solução 1.

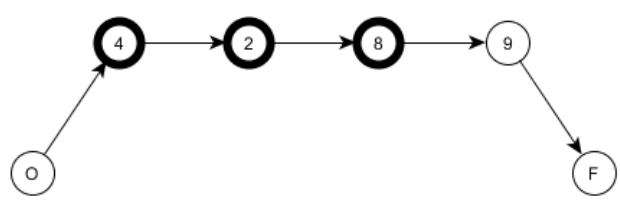

(b) Bloco e caminho crítico solução 2.

Figura 7 - Blocos do caminho crítico.

No caso do JS, a estratégia de ramificação utilizada por Brucker, Jurisch e Sievers (1994) tem como suporte o teorema 5:

Teorema 5. (BRUCKER; JURISCH; SIEVERS, 1994) Seja S uma seleção completa correspondente a alguma solução factivel para o JS. Se existe uma seleção completa $S^{\prime}$ tal que $L\left(S^{\prime}\right)<L(S)$, então pelo menos uma operação de algum bloco em $G_{S}$ será processada antes da primeira operação ou após a última operação do correspondente bloco.

Demonstração: Dada a seleção completa e consistente $S$, considere o caminho crítico $P_{S}$ em $G_{S}=(V, C \cup S)$ definido pela sequência de operações

$$
P_{S}=\left(O, u_{1}^{1}, \ldots, u_{m_{1}}^{1}, \ldots, u_{1}^{k}, \ldots, u_{m_{k}}^{k}, F\right)
$$

em que $B_{j}=\left(u_{1}^{j}, \ldots, u_{m_{j}}^{j}\right)$ representa o $j$-ésimo bloco de $P_{S}$. Seja $S^{\prime}$ uma seleção completa e consistente tal que $L\left(S^{\prime}\right)<L(S)$ e suponha por absurdo que nenhuma operação de qualquer bloco $B_{j}$ de $P_{S}$ é processada antes da primeira ou após a última operação do correspondente bloco. Portanto, sem eliminar os arcos redundantes, o grafo $G_{S}^{\prime}=\left(V, C \cup S^{\prime}\right)$ contém os arcos

$$
\begin{aligned}
& u_{1}^{j} \rightarrow u_{i}^{j}, \quad j=1, \ldots, k, \quad i=2, \ldots, m_{j}, \\
& u_{i}^{j} \rightarrow u_{m_{j}}^{j}, \quad j=1, \ldots, k, \quad i=1, \ldots,\left(m_{j-1}\right) \mathrm{e} \\
& u_{m_{j}}^{j} \rightarrow u_{1}^{j+1}, \quad j=1, \ldots, k-1 \text { (arcos conjuntivos). }
\end{aligned}
$$

Dessa forma, $G_{S}^{\prime}$ contém o caminho

$$
C=\left(O, u_{1}^{1}, \pi_{2}^{1}, \ldots, \pi_{\left(m_{1}-1\right)}^{1}, u_{m_{1}}^{1}, \ldots, u_{1}^{k}, \pi_{2}^{k}, \ldots, \pi_{\left(m_{1}-1\right)}^{k}, u_{m_{k}}^{k}, F\right)
$$

em que a sequência $\pi_{2}^{j}, \ldots, \pi_{\left(m_{1}-1\right)}^{j}$ representa uma permutação das operações $u_{2}^{j}, \ldots, u_{\left(m_{1}-1\right)}^{j}$ do bloco $B_{j}$. O comprimento do caminho crítico em $G\left(S^{\prime}\right)$ é maior ou igual que o comprimento 
do caminho $C$, uma vez que o caminho crítico representa o caminho de maior valor entre as operações $O$ e $F$. Logo, denotando $\pi_{1}^{j}=u_{1}^{j}$ e $\pi_{m_{j}}^{j}=u_{m_{j}}^{j}$, para $j=1, \ldots, k$, temos que

$$
\begin{aligned}
L\left(S^{\prime}\right) & \geq L(C) \\
& =\sum_{j=1}^{k} \sum_{i=1}^{m_{j}} p_{\pi_{i}^{j}} \\
& =\sum_{j=1}^{k} \sum_{i=1}^{m_{j}} p_{u_{i}^{j}} \\
& =L(S) .
\end{aligned}
$$

Entretanto, isto contradiz nossa hipótese. Portanto, se $L\left(S^{\prime}\right)<L(S)$, então pelo menos a posição da primeira ou da última operação de algum bloco $B_{j}$ deverá ser alterada.

Voltando as seleções do JS apresentadas nas figuras 5a e 5b, temos que $L\left(S_{2}\right)<L\left(S_{1}\right)$, em que $S_{1}$ e $S_{2}$ representam as seleções completas e consistentes associadas às soluções 1 e 2 , respectivamente. Portanto, de acordo com o teorema 5, pelo menos uma operação do bloco (2-8-4) (único bloco do caminho crítico da solução 1) deve ser executada antes da operação 2 ou após a operação 4 no sequenciamento definido pela solução 2. E de fato, na solução 2 temos que a operação 4 é executada antes da operação 2 (veja a Figura 4-(b)).

\subsection{Job-shop flexível}

Ao contrário do JS, em que cada operação é executada por uma única máquina, no problema do job-shop flexível (JSF), uma operação pode ser processada por um conjunto de máquinas. Nesse contexto, o termo flexibilidade significa a habilidade de usar diferentes máquinas para processar algumas ou todas as operações de uma tarefa. Dessa forma, o JSF é uma extensão do JS.

Além de incorporar todas as dificuldades do JS, o JSF é mais complexo devido à flexibilidade entre as máquinas. Assim, a resolução do JSF é, pelo menos, tão difícil quanto o JS, logo, ele também é considerado um problema NP-difícil (BIRGIN et al., 2014).

Como no JSF cada operação pode ser processada em diferentes máquinas, a sua resolução pode ser dividida em duas etapas: uma etapa de roteamento e outra de sequenciamento. A primeira delas consiste em um subproblema para atribuir cada operação a um conjunto de máquinas habilitadas a processá-la. Definidas as atribuições, o segundo subproblema compreende definir o sequenciamento dessas operações em cada máquina.

Conforme a quantidade de máquinas habilitadas a processar uma operação, Kacem, Hammadi e Borne (2002) classificaram a flexibilidade do problema em duas categorias: total e parcial. Para a flexibilidade total, cada operação pode ser processada por todas as máquinas e a flexibilidade parcial indica que cada operação pode ser processada apenas por um subconjunto do total de máquinas.

Embora o desenvolvimento de métodos para a resolução JS tenha sido impulsionado após a publicação do livro Industrial Scheduling, em 1963, o primeiro caso de estudo para JSF ocorreu em 1990 com o trabalho de Brucker e Schlie (1990) (CHAUDHRY; KHAN, 2015). Os autores desenvolveram um algoritmo em tempo polinomial para o caso do JSF com apenas duas tarefas. 
O JSF descreve uma grande variedade de problemas encontrados em sistemas de manufatura, uma vez que, com as exigências do mercado e com o avanço tecnológico, muitas das máquinas presentes nas indústrias têm a propriedade de realizar mais de um tipo diferente de tarefa. Dependendo da situação, uma modelagem mais realista do JSF deve levar em consideração hipóteses adicionais na configuração do problema. Há uma grande variedade de restrições, sendo que as principais são descritas na sequência:

- Restrições de atividades de manutenção. Nesse tipo de situação, as máquinas podem ficar indisponíveis durante um determinado período do horizonte de planejamento. A indisponibilidade da máquina pode ocorrer em decorrência de avarias, de manutenção ou de controle. O JSF com restrições de manutenção pode ser encontrado nos trabalhos de Gao, Gen e Sun (2006), Rajkumar, Asokan e Vamsikrishna (2010), Wang e Yu (2010) e Li, Pan e Tasgetiren (2014).

- Incerteza no tempo de processamento das máquinas. No JSF, o tempo de processamento de uma operação é um valor determinístico. Entretanto, como as máquinas têm múltiplas funções, pode ser que o tempo de processamento não seja conhecido com precisão. Assim sendo, o tempo de processamento é representado por um número difuso e o problema é conhecido como JSF Difuso (Fuzzy Flexible Job-Shop). Mais detalhes do JSF Difuso podem ser encontrados nos trabalhos de Fortemps (1997), Lei (2010), Lei (2012) e Palacios et al. (2014).

- Diferentes planos de processo. O JSF consiste em uma sequência de operações que devem ser executadas em uma determinada ordem. O plano de processo estabelece a ordem de processamento dessas operações para cada tarefa. A versão do JSF com múltiplos planos de processo estabelece diferentes alternativas para as restrições de precedências entre as operações. Assim, o problema permite que operações de uma mesma tarefa sejam processadas de forma simultânea. Doh et al. (2013) descrevem uma heurística baseada em regra de despacho para o problema, Özgüven, Özbakır e Yavuz (2010) e Birgin et al. (2014) apresentam uma formulação por MILP.

- Tempo para configuração das máquinas. Quando uma operação é executada, pode ser necessário aplicar alguma configuração específica na máquina habilitada a processála. De forma geral, o tempo de configuração, ou setup time, é incorporado ao tempo de processamento da operação ou ignorado. Embora esse procedimento simplifique a análise em certas aplicações, ele afeta negativamente a qualidade da solução para muitas situações que requerem tratamento explícito dessa defasagem de tempo (ROSSI, 2014). Por isso, tem aumentado o interesse em incluir de forma explícita as restrições de configuração das máquinas ao JSF. Métodos heurísticos para a resolução dessa versão do JSF podem ser encontrados, por exemplo, em Yu e Ram (2006), Rossi e Dini (2007) e Defersha e Chen (2010). Özgüven, Yavuz e Özbakır (2012) e Nourali, Imanipour e Shahriari (2012) apresentam duas formulações por MILP para o problema.

Em decorrência da complexidade do JSF, muitas vezes é preferível obter uma solução razoável em um período de tempo mais curto do que esperar dias ou semanas para obter uma solução ótima. Logo, métodos heurísticos e metaheurísticos para o JSF são amplamente estudados e a quantidade de trabalhos que buscam resolver esses problemas através de métodos exatos é pequena (BIRGIN et al., 2014). Os principais métodos exatos encontrados na literatura para o JSF são baseados na utilização de resolvedores em formulações lineares, não lineares de programação inteira mista e programação por restrições. 
Embora os métodos exatos possam não ser uma solução eficiente para o JSF do ponto de vista prático, eles podem ser o primeiro passo antes de desenvolver heurísticas eficazes e úteis para a compreensão da estrutura do problema (DEMIR; İŞLEYEN, 2013). Métodos exatos podem ser capazes de fornecer, senão uma solução ótima, limitantes satisfatórios para muitos problemas. A procura de bons limitantes inferiores nos ajuda a resolver uma das principais dificuldades do método B\&B que é de podar a árvore de busca o mais cedo possível. Além disso, limitantes superiores permitem avaliar a qualidade de métodos heurísticos.

Um trabalho recente que aborda técnicas de resolução para o JSF foi publicado em 2015 por Chaudhry e Khan (2015). Neste trabalho, a partir da seleção de 191 artigos, os autores descrevem o panorama das abordagens utilizadas para a resolução do JSF durante o período de Janeiro de 1990 à Fevereiro de 2014. Por ser o primeiro a abordar de forma ampla as técnicas de resolução, descrevemos, de forma resumida, os principais resultados obtidos pela pesquisa:

- Número de publicações. O período de 1990 a 2009 representa apenas 36,46\% das publicações. O grande crescimento ocorreu durante o período de 2010 a 2013, com 123 publicações $(64,39 \%)$.

- Função Objetivo. Em 44,67\% dos artigos, o makespan é utilizado como a única medida de desempenho e em 39,59\% o makespan é utilizado em combinação com outras funções objetivo. O atraso total aparece em 1,52\% dos trabalhos e o atraso médio em $1,02 \%$. Os demais trabalhos consistem da combinação de duas ou mais medidas de desempenho.

- Técnicas de Resolução. As técnicas de resolução foram divididas em 14 categorias: otimização por Colônia de Formigas, otimização por Colônia de Abelhas Artificiais, Sistemas Imunológicos Artificiais, algoritmos Evolutivos, GRASP (Greedy Randomized Adaptative Search Procedure), Programação Linear e Inteira, Busca em Vizinhança, otimização por Nuvem de Partículas, Recozimento Simulado, Programação Matemática, Heurísticas Determinísticas, Técnicas Híbridas e Miscelâneas de Técnicas. A Tabela 2 descreve o número de artigos e a proporção da utilização destas técnicas.

Técnicas híbridas representam 35,03\% das publicações. Essa técnica combina uma ou mais heurísticas ou metaheurísticas para tirar proveito de seus pontos fortes para obter a melhor solução. Em segundo lugar, aparecem os Algoritmos Evolutivos, com 23,86\% das publicações. Somente 10 trabalhos apresentam formulações para o problema. Embora não seja relatado no trabalho original, observamos que desses 10 trabalhos, 2 apenas apresentam a formulação mas utilizam abordagens heurísticas e metaheurísticas em sua resolução. Nos 8 trabalhos restantes, os autores utilizam resolvedores para obter a solução ótima ou limitantes.

- Variações do JSF. A maioria dos artigos abordaram a versão padrão do JSF e somente 35,53\% consideraram diferentes cenários e configurações. Do total de 191 artigos, somente em 6,28\% (12 artigos) o problema é oriundo de uma aplicação real, sendo que os demais focam no desenvolvimento de técnicas de resolução.

Em termos de formulações por MILP, as principais são devidas aos trabalhos de Fattahi, Mehrabad e Jolai (2007), Özgüven, Özbakır e Yavuz (2010) e Birgin et al. (2014). Fattahi, Mehrabad e Jolai (2007) desenvolveram um modelo por programação inteira com o objetivo de minimizar o makespan e dois conjuntos de heurísticas. Embora seja classificado 
Tabela 2 - Técnicas utilizadas para a resolução do JSF.

\begin{tabular}{lll}
\hline Técnica Utilizada & Número de Artigos & Porcentagem (\%) \\
\hline Técnicas Híbridas & 69 & 35,03 \\
Algoritmos Evolutivos & 47 & 23,86 \\
Heurísticas Determinísticas & 19 & 9,64 \\
Busca Tabu & 12 & 6,09 \\
Programação Linear e Inteira & 10 & 5,08 \\
Nuvem de Partículas & 8 & 4,06 \\
Miscelânea de Técnicas & 7 & 3,55 \\
Busca em Vizinhança & 6 & 3,05 \\
Sistemas Imunológicos Artificiais & 5 & 2,54 \\
Programação Matemática & 4 & 2,03 \\
Recozimento Simulado & 4 & 2,03 \\
Colônia Formigas & 3 & 1,52 \\
GRASP & 2 & 1,02 \\
Colônia de Abelhas & 1 & 0,51 \\
\hline
\end{tabular}

Fonte: Chaudhry e Khan (2015)

pelos autores como um MILP, o modelo apresenta em uma de suas restrições o produto de uma variável contínua com uma variável binária (ou o produto de duas variáveis binárias, dependendo da combinação das restrições). Além disso, os autores propõem um conjunto de 20 instâncias teste para o JSF. Esses 20 problemas são divididos em 10 instâncias de pequeno porte e 10 de médio e grande porte. O modelo é utilizado para alcançar a solução ótima para problemas de tamanhos menores.

Özgüven, Özbakır e Yavuz (2010) abordam o JSF e o JSF com flexibilidade no processo de sequenciamento. Inicialmente foi proposto um MILP para o JSF com base no modelo de Manne (1960) e comparado com o modelo proposto por Fattahi, Mehrabad e Jolai (2007). O modelo foi testado através da resolução de 20 instâncias mostrando melhor desempenho. A partir do primeiro modelo, é apresentado um novo MILP com flexibilidade no processo de sequenciamento, e este é o primeiro modelo da literatura.

Uma análise das principais formulações para o JSF é apresentada por Demir e İşleyen (2013). Com base no tipo de variável binária utilizada para descrever a etapa de sequenciamento, quatro formulações utilizadas na literatura são apresentadas: Fattahi, Mehrabad e Jolai (2007), Özgüven, Özbakır e Yavuz (2010), Gao, Gen e Sun (2006) e Kim e Egbelu (1999). Os autores também propõem um modelo com variáveis indexadas no tempo. Os experimentos computacionais avaliaram os modelos conforme o valor da função objetivo, o tempo computacional gasto na resolução das instâncias, o número de variáveis e o número de restrições. Como resultado, os autores recomendam a utilização de modelos caracterizados pelas variáveis de precedência descritas por Manne (1960), e, em especial, o modelo proposto por Özgüven, Özbakır e Yavuz (2010).

Uma nova formulação para o JSF com o objetivo de minimizar o makespan é apresentada por Birgin et al. (2014). Essa nova formulação também permite descrever situações em que operações que pertencem a uma mesma tarefa necessitam ser processadas simultaneamente. Dessa forma, as restrições de precedências entre as operações de uma mesma tarefa podem ser representadas por uma grafo acíclico dirigido em vez de uma ordenação linear. Exemplos dessas situações podem ser encontrados, por exemplo, em fábricas de vidros e na 
indústria gráfica. Os autores comparam com o modelo proposto por Özgüven, Özbakır e Yavuz (2010) usando instâncias da literatura e instâncias oriundas de uma indústria gráfica. Os resultados computacionais mostraram que o modelo novo é melhor que o modelo de Özgüven, Özbakır e Yavuz (2010).

Em relação ao uso de inequações válidas para o JSF, o único trabalho encontrado na literatura é a dissertação de mestrado de Leffler (2015). A pesquisa apresenta um conjunto de inequações válidas para JSF e variáveis indexadas no tempo são utilizadas para a formulação do problema. O autor apenas utilizou as inequações válidas em um algoritmo de PC para avaliar a qualidade dos limitantes inferiores. Nos experimentos computacionais, foram utilizados 20 problemas propostos por Fattahi, Mehrabad e Jolai (2007) e a solução obtida pelo algoritmo PC foi comparada com a solução obtida pela relaxação do problema. Os resultados mostraram que, em algums casos, as inequações válidas melhoram a qualidade do limitante inferior. Como veremos nos experimentos computacionais, das 20 instâncias propostas por Fattahi, Mehrabad e Jolai (2007), em 18 a solução ótima é obtida com tempo inferior a 225 segundos. Comparados com outros conjuntos de instâncias teste, o proposto por Fattahi, Mehrabad e Jolai (2007) é de pequeno porte. O autor não utilizou outros conjuntos de problemas nos experimentos pois a formulação por variáveis indexadas no tempo tende a aumentar rapidamente com o tamanho do problema e esse é um dos pontos fracos desse tipo de formulação.

\subsubsection{Descrição do job-shop flexível e formulação matemática (MILP-1)}

A modelagem do JSF através de um grafo disjuntivo não é uma tarefa viável, uma vez que existem operações que podem ser executadas por várias máquinas. Dessa forma, o JSF tem sido descrito através de modelos por programação inteira mista (linear e não-linear) por vários autores na literatura. Conforme comentado anteriormente, o JSF é composto por dois subproblemas: roteamento e sequenciamento. Para a formulação do problema, na etapa de roteamento é introduzida uma variável binária $x_{i j k}$ que recebe o valor 1 se a operação $O_{i j}$ é processada na máquina $k$ e 0 , caso contrário. Para o subproblema de sequenciamento, os modelos por MILP podem ser classificados conforme a característica da variável binária utilizada para representar essa etapa (DEMIR; İŞLEYEN, 2013). A classificação é baseada em três tipos de variáveis:

- Variável baseada na sequência-posição. Esse tipo de variável foi proposta por Wagner (1959) e define a posição em que uma operação será sequenciada no roteiro de programação de cada máquina. As variáveis são definidas com base na quantidade de operações que uma determinada máquina pode processar. Essa quantidade de operações representa as possíveis posições em que uma operação possa ser designada e, portanto, estabelece a sua ordem de sequenciamento em relação às demais. Assim, a variável de decisão $z_{i j k l}$ recebe o valor 1 se a operação $O_{i j}$ é designada na máquina $k$ na posição $l$ e 0, caso contrário. Fattahi, Mehrabad e Jolai (2007), Fattahi, Jolai e Arkat (2009) e Khalife, Abbasi e Abadi (2010) utilizaram esse tipo de variável para formular o JSF.

- Variável de precedência. Denota a sequência de operações atribuídas a uma mesma máquina. Se a operação $O_{i j}$ precede (não necessariamente de forma imediata) a operação $O_{i^{\prime} j^{\prime}}$ na máquina $k$, então a variável binária $y_{i j, i^{\prime} j^{\prime}, k}$ recebe o valor 1 . Caso contrário, $y_{i j, i^{\prime} j^{\prime}, k}=0$. Esse tipo de variável foi introduzida por Manne (1960) para o problema de sequenciamento em uma única máquina com restrições de precedência e pode ser 
encontrada, por exemplo, nos trabalhos de Kim e Egbelu (1999), Gao, Gen e Sun (2006), Özgüven, Özbakır e Yavuz (2010) e Birgin et al. (2014).

- Variável indexada no tempo. Nessa situação, o horizonte de planejamento é discretizado em um número inteiro de períodos de igual duração. Esse modelo de variável foi introduzido por Bowman (1959) para a formulação matemática do JS, até então denominado de Problema de Programação do Sequenciamento (schedule-sequencing problem). A variável de decisão $w_{i j u k}$ utilizada por Bowman recebe o valor 1 se a operação $O_{i j}$ é processada no tempo $t=u$ e na máquina $k$ e 0 , caso contrário. Formulações para JSF utilizando variáveis indexadas no tempo estão presentes nos trabalhos de Demir e İşleyen (2013) e Thörnblad et al. (2013).

Basicamente essas três classificações são as que definem e distinguem as principais formulações para o JSF na literatura.

Em seguida, descrevemos o JSF. Sejam $T=\left\{T_{1}, \ldots, T_{n}\right\}$ o conjunto de $n$ tarefas e $M=\left\{M_{1}, \ldots, M_{m}\right\}$ o conjunto de $m$ máquinas. Para cada tarefa $i \in T$, seja $O_{i}$ o conjunto de $n_{i}$ operações a serem processadas em sequência:

$$
O_{i 1} \rightarrow O_{i 2} \rightarrow \cdots \rightarrow O_{i n_{i}}
$$

Cada operação $O_{i j}$ pode ser processada por um conjunto de máquinas $\mathscr{M}_{i j} \subseteq M$. O tempo de processamento da operação $O_{i j}$ na máquina $k \in \mathscr{M}_{i j}$ é denotado por $p_{i j k}$.

Para exemplicar, a Tabela 3 descreve uma instância para o JSF com 3 tarefas, 3 máquinas e 8 operações. Nesse exemplo de flexibilidade parcial, as operações $O_{12}, O_{13}, O_{21}$ e $O_{31}$ são processadas em uma única máquina, enquanto as operações $O_{11}, O_{22}$ e $O_{33}$ podem ser processadas em duas máquinas distintas. Para a operação $O_{11}$, o tempo de processamento da máquina 1 é igual a 2 minutos $\left(p_{111}=2\right)$ e o tempo de processamento na máquina 2 é igual a 5 minutos $\left(p_{112}=5\right)$.

Tabela 3 - Instância para o JSF com 3 tarefas e 3 máquinas.

\begin{tabular}{ccccc}
\hline Tarefa & Operação & Máquina $1(\min )$ & Máquina $2(\min )$ & Máquina $3(\mathrm{~min})$ \\
\hline \multirow{3}{*}{1} & $O_{11}$ & 2 & 5 & - \\
& $O_{12}$ & - & 3 & - \\
& $O_{13}$ & - & - & 4 \\
\hline \multirow{2}{*}{2} & $O_{21}$ & 1 & - & - \\
& $O_{22}$ & - & 3 & 2 \\
\hline \multirow{3}{*}{3} & $O_{31}$ & 1 & - & - \\
& $O_{32}$ & - & 3 & - \\
& $O_{33}$ & - & 3 & 5 \\
\hline
\end{tabular}

A atribuição das operações para cada máquina pode ser descrita por uma função de mapeamento $\mu: O \rightarrow M$, tal que $\mu\left(O_{i j}\right)$ se refere à máquina em que $O_{i j}$ será processada e $\mu\left(O_{i j}\right) \in \mathscr{M}_{i j}$, para todo $i=1, \ldots, m$ e $j=1, \ldots, n_{i}$. Além disso, o domínio da função $\mu$ representa o conjunto formado pelas operações de todas as tarefas. Definida uma atribuição $\mu$, o JSF se torna o clássico JS. Assim, a atribuição $\mu$ induz a representação do problema por grafo disjuntivo $G=\left(V, C \cup D^{\mu}\right)$. As definições dos conjuntos $V, A$ e $D^{\mu}$ são as mesmas utilizadas na seção 2.2.1 para descrever o JS. 
Voltando ao exemplo descrito pela Tabela 3, suponha que a função atribuição $\mu$ seja definida por: $\mu\left(O_{11}\right)=1, \mu\left(O_{22}\right)=3, \mu\left(O_{33}\right)=3$ e para as demais operações $\mu$ atribui a única máquina possível para processá-la. O grafo disjuntivo $G=\left(V, C \cup D^{\mu}\right)$ associado à função $\mu$ é representado pela Figura 8. Logo, dada a função atribuição $\mu$ e uma seleção completa e consistente $S$, o par $(\mu, S)$ representa uma solução factível para o JSF. Denotamos o grafo associado ao par $(\mu, S)$ por $G_{(\mu, S)}=\left(V, C \cup S^{\mu}\right)$ e o valor do caminho crítico em $G_{(\mu, S)}$ por $L\left(S^{\mu}\right)$.
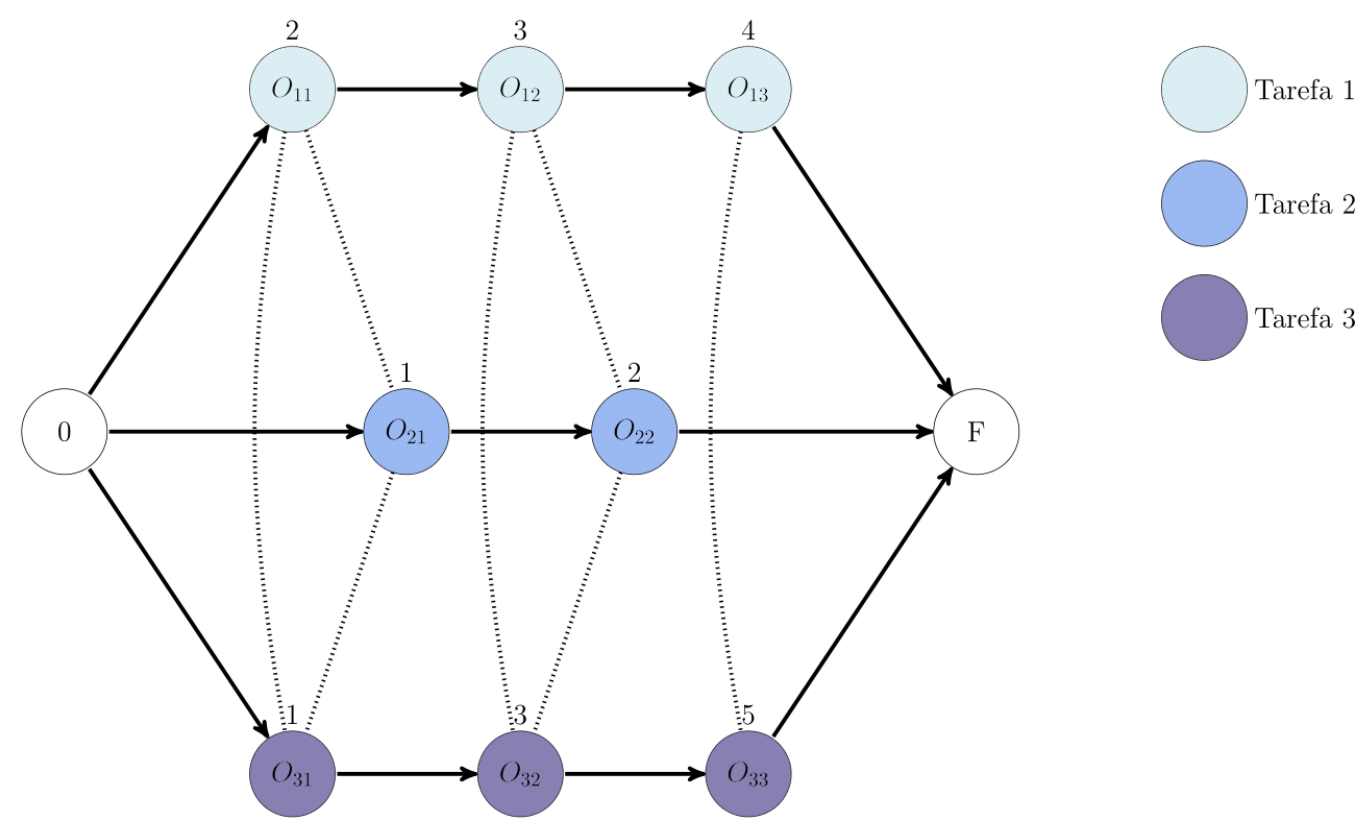

Figura 8 - Grafo disjuntivo associado a atribuição $\mu$.

O próximo teorema é uma generalização do teorema 5.

Teorema 6. (BRUCKER; SCHLIE, 2006, p.210) Sejam y e $y^{\prime}$ duas soluções factíveis do JSF associadas a $(\mu, S)$ e $\left(\mu^{\prime}, S^{\prime}\right)$, respectivamente. Se $L\left(S^{\prime \mu^{\prime}}\right)<L\left(S^{\mu}\right)$, então

i) em $\mu^{\prime}$, pelo menos uma operação de algum bloco $B$ do caminho crítico de $G_{(\mu, S)}$ é atribuída para outra máquina, ou

ii) em $S^{\prime}$, pelo menos uma operação de algum bloco $B$ do caminho crítico de $G_{(\mu, S)}$ tem que ser processada antes da primeira ou após a última operação de $B$.

Demonstração: Considere o caminho crítico $P_{(\mu, S)}$ em $G_{(\mu, S)}$ definido pela sequência de operações

$$
P_{(\mu, S)}=\left(O, u_{1}^{1}, \ldots, u_{m_{1}}^{1}, \ldots, u_{1}^{k}, \ldots, u_{m_{k}}^{k}, F\right) .
$$

Cada conjunto $B_{j}=\left(u_{1}^{j}, \ldots, u_{m_{j}}^{j}\right), j=1, \ldots, k$, representa um bloco em $P_{(\mu, S)}$. Vamos supor por absurdo que em $y^{\prime}$ as operações de todos os blocos de $P_{(\mu, S)}$ são processadas na mesma máquina que as definidas em $y$, e que em $y^{\prime}$ nenhuma operação de qualquer bloco $B_{j}$ em $P_{(\mu, S)}$ é processada antes da primeira ou após a última operação do correspondente bloco. Logo, em $G_{\left(\mu^{\prime}, S^{\prime}\right)}$ também temos que

- se $m_{j} \geq 2$, então $u_{1}^{j}$ é processada antes de $u_{2}^{j}, \ldots, u_{m_{j}}^{j}$ e $u_{m_{j}}^{j}$ é processada após as operações $u_{1}^{j}, \ldots, u_{m_{j-1}}^{j}(j=1, \ldots, k)$, 
- $u_{m_{j}}^{j}$ é processada antes de $u_{1}^{j+1}(j=1, \ldots, k-1)$, pois $\left(u_{m_{j}}^{j}, u_{1}^{j+1}\right)$ representa um arco conjuntivo.

Assim, $G_{\left(\mu^{\prime}, S^{\prime}\right)}$ contém o caminho

$$
C=\left(O, \pi_{1}^{1}, \pi_{2}^{1}, \ldots, \pi_{\left(m_{1}-1\right)}^{1}, \pi_{m_{1}}^{1}, \ldots, \pi_{1}^{k}, \pi_{2}^{k}, \ldots, \pi_{\left(m_{1}-1\right)}^{k}, \pi_{m_{k}}^{k}, F\right),
$$

em que a sequência $\pi_{2}^{j}, \ldots, \pi_{\left(m_{1}-1\right)}^{j}$ representa uma permutação das operações $u_{2}^{j}, \ldots, u_{\left(m_{1}-1\right)}^{j}$ do bloco $B_{j}$ e $\pi_{1}^{j}=u_{1}^{j}$ e $\pi_{m_{j}}^{j}=u_{m_{j}}^{j}$, para $j=1, \ldots, k$.

Como o comprimento do caminho crítico em $G_{\left(\mu^{\prime}, S^{\prime}\right)}$ é maior ou igual ao comprimento do caminho $C$ e as operações $\pi_{1}^{j}, \ldots, \pi_{m_{j}}^{j}$ são processadas na mesma máquina que em $y$, então

$$
\begin{aligned}
L\left(S^{\prime \mu^{\prime}}\right) & \geq L(C) \\
& =\sum_{j=1}^{k} \sum_{i=1}^{m_{j}} p_{\pi_{i}^{j}} \\
& =\sum_{j=1}^{k} \sum_{i=1}^{m_{j}} p_{u_{i}^{j}} \\
& =L\left(S^{\mu}\right) .
\end{aligned}
$$

Mas a inequação acima apresenta uma contradição, pois pela hipótese, temos que $L\left(S^{\prime \mu^{\prime}}\right)<$ $L\left(S^{\mu}\right)$.

O teorema 6 é uma ferramenta importante para estabelecer estruturas de vizinhanças em metaheurísticas que utilizam o procedimento de busca local. Geralmente, um vizinho de uma solução factível é obtido realizando algum tipo de movimento ou operação. Por exemplo, vizinhos de uma solução factível podem ser criados alterando a ordem do sequenciamento da primeira operação de cada bloco do caminho crítico ou atribuindo as operações de bloco crítico para outras máquinas. Os trabalhos de Brandimarte (1993), Najid, Dauzere-Pérès e Zaidat (2002) e Fernández, Vela e Arias (2013) utilizam as propriedades do teorema 6 para estabelecer diversas estruturas de vizinhanças para a resolução do JSF. Nesta tese, o teorema será utilizado no capítulo 4 (seção 4.4.2) em uma das implementações do método Diversification, Refining, and Tight-Refining (FISCHETTI; POLO; SCANTAMBURLO, 2004).

O modelo utilizado para descrever o JSF é o proposto por Birgin et al. (2014). O modelo é baseado na variáveis de precedências descritas por Manne (1960). Adotamos esse modelo em decorrência da eficiência dos resultados obtidos pelos experimentos computacionais descritos no trabalho dos autores. No decorrer do texto, denominamos esse modelo de MILP-1.

No modelo MILP-1, não é utilizada uma lista ordenada de operações que compõem cada tarefa, mas um conjunto de todas as operações do problema e suas relações de precedência. Dessa forma, o modelo foca nas operações e nas suas relações de precedências e deixa a tarefa dessas operações definida de forma implícita. Isso também permite que o modelo descreva situações em que operações de uma mesma tarefa sejam executadas ao mesmo tempo, complementando a versão padrão em que operações são descritas de forma sequencial. A notação $O_{i j}$ é substituída por $O_{i}$ e o índice $j$ também será utilizado para referenciar uma operação. O número total de operações é definido por $N=\sum_{i=1}^{n} n_{i}$ e a ordem de processamento das operações em uma mesma máquina é definida pela variável $y_{i j}$. Para descrever o modelo, considere, além das variáveis descritas no início dessa seção, os seguintes dados: 
- O: conjunto de todas as operações de todas as tarefas;

- $i, j$ : índices para o conjunto $O(1 \leq i \leq N, 1 \leq j \leq N)$;

- $k$ : índice para o conjunto $M(1 \leq k \leq m)$;

- $\mathcal{O}_{k}$ : conjunto de operações que podem ser processadas na máquina $k\left(\mathcal{O}_{k} \subseteq O\right)$;

- $\mathscr{M}_{i}$ : conjunto de máquinas habilitadas a processar a operação $i\left(\mathscr{M}_{i} \subseteq M\right)$;

- $E_{k}$ : conjunto de pares ordenados de elementos distintos de $\mathcal{O}_{k}\left(E_{k} \subseteq \mathcal{O}_{k} \times \mathcal{O}_{k}\right)$;

- $E=\cup_{k \in M} E_{k}$;

- $P$ : conjunto de pares ordenados correspondente à restrição de precedência entre duas operações $(P \subseteq O \times O)$. Assim, $(i, j) \in P$ se e somente se a operação $i$ precede a operação $j$ em alguma tarefa;

- $L$ : constante inteira suficientemente grande;

- $p_{i k}$ : tempo de processamento da operação $i$ na máquina $k$;

- $x_{i k}$ : variável binária que assume o valor 1 se a operação $i$ é processada na máquina $k$ e zero, caso contrário;

- $y_{i j}$ : variável binária que assume o valor 1 se a operação $i$ precede $j$ e ambas são processadas na mesma máquina. Caso contrário, a variável pode assumir o valor 1 ou 0;

- $s_{i}$ : instante de início do processamento da operação $i$;

- $p_{i}$ : tempo de processamento da operação $i$ após a atribuição à máquina;

- $C_{M a x}$ : instante de conclusão da última tarefa (makespan).

A função objetivo consiste em minimizar o makespan. Assim, se $c_{i}=s_{i}+p_{i}$ representa o instante para finalizar a operação $i$, então o objetivo do problema é minimizar o maior valor para $c_{i}$, para $i \in O$, isto é, $\min \left[\max _{i \in O} \quad c_{i}\right]=\min C_{M a x}$. Portanto, o JSF pode ser descrito por:

$$
\begin{aligned}
& \text { Minimizar } C_{M a x} \\
& \text { Sujeito a } \sum_{k \in \mathscr{M}_{i}} x_{i k}=1, \quad \forall i \in O \\
& p_{i}=\sum_{k \in \mathscr{M}_{i}} x_{i k} \cdot p_{i k}, \quad \forall i \in O \\
& C_{\text {Max }} \geq s_{i}+p_{i}, \quad \forall i \in O \\
& y_{i j}+y_{j i} \geq x_{i k}+x_{j k}-1, \quad \forall k \in M, \forall(i, j) \in E_{k} \\
& s_{i}+p_{i}-L \cdot\left(1-y_{i j}\right) \leq s_{j}, \quad \forall(i, j) \in E \\
& s_{i}+p_{i} \leq s_{j}, \quad \forall(i, j) \in P \\
& s_{i} \geq 0, \quad \forall i \in O \\
& x_{i k} \in\{0,1\}, \quad \forall k \in M, \forall i \in \mathcal{O}_{k} \\
& y_{i j} \in\{0,1\}, \quad \forall(i, j) \in E \text {. }
\end{aligned}
$$


A função objetivo é descrita em (2.9). A restrição do tipo (2.10) assegura que uma operação é executada somente em uma única máquina. A restrição (2.11) determina o tempo de processamento de cada operação pela máquina selecionada. A restrição (2.12) assegura que o valor do makespan é sempre maior ou igual ao instante de término de qualquer operação. A restrição (2.13) define a ordem de procedência entre duas operações, caso estas sejam atribuídas a uma mesma máquina, e neste caso, (2.14) estabelece que estas não podem ser processadas simultaneamente. Observe que, se $x_{i k}=x_{j k}=1$, então as restrições (2.13) e (2.14) forçam a decidir se a operação $i$ é executada antes ou depois de $j$. Caso contrário, a restrição (2.13) é redundante. O valor da constante $L$ deve ser suficientemente grande para que a restrição (2.14) seja válida caso $y_{i j}=0$. A restrição (2.15) assegura que a restrição de precedência entre operações de uma mesma tarefa não seja violada. As variáveis do problema são descritas pelas restrições (2.16)-(2.18).

\subsection{Resumo}

Neste capítulo apresentamos o JS e JS com a propriedade de flexibilidade entre as máquinas (JSF). Após descrevermos as principais características dos problemas de scheduling, expomos as definições, propriedades e formulações do JS e JSF. 


\section{Capítulo 3}

\section{Inequações válidas para o job-shop fle- xível}

Neste capítulo, buscamos cumprir um dos objetivos desta tese: apresentar um conjunto de inequações válidas para o JSF e analisar a sua eficiência em um algoritmo B\&C.

As inequações válidas foram definidas em dois contextos. O primeiro contexto está relacionado com as permutações das operações que são atribuídas a uma mesma máquina e o segundo são adaptações das inequações para o JS. Dessa forma, antes de apresentar as novas inequações válidas, descrevemos as inequações já definidas para o JS.

Na segunda parte deste capítulo, apresentamos os experimentos computacionais. Os experimentos foram divididos em três partes. A primeira parte aborda o conjunto de instâncias teste, a segunda analisa a eficiência das diversas classes de inequações válidas através de um algoritmo de $\mathrm{PC}$ e a última parte apresenta os resultados do algoritmo de B\&C.

\subsection{Inequações válidas para o job-shop}

As inequações válidas apresentadas nesta tese utilizam adaptações das inequações descritas por Applegate e Cook (1991) para o caso do JS. Nesta seção, descrevemos as classes de inequações válidas para o JS utilizadas no trabalho de Applegate e Cook (1991). Para cada classe de inequações válidas, os autores apresentam um nova família de inequações, denominada de reversa, ao considerar a ordem inversa do sequenciamento das operações. Não descrevemos todas as classes de inequações, mas apenas as que serão estendidas para o ambiente do JSF.

Para descrevê-las, precisamos inicialmente definir dois conceitos importantes relacionados a toda operação $i$ : head $\left(r_{i}\right)$ e tail $\left(q_{i}\right)$. O valor $r_{i}$ representa o instante de disponibilidade da operação $i$ na entrada do sistema. Embora as operações estejam disponíveis no instante $t=0$, devido às restrições de precedência, $r_{i}$ é definido pela soma dos tempos de processamento das operações que necessariamente a antecedem. O valor $q_{i}$ é o menor tempo necessário para completar a tarefa a qual a operação $i$ pertence, após o seu processamento. Assim, $q_{i}$ é definido pela soma dos tempos de processamento das operações que a sucedem. Considere $a^{+}$ como sendo o máximo de $a$ e 0 , para todo número real $a$.

$\mathrm{Na}$ sequência, apresentamos as inequações válidas que foram analisadas neste trabalho. Quando necessário, utilizamos a notação adotada no Capítulo 2.

Propriedade 1 (Two Jobs). Sejam $i$ e $j$ duas operações executadas em uma mesma 
máquina $k \in M$. Se $r_{j}<r_{i}+p_{i}$ e $r_{i}<r_{j}+p_{j}$, então o corte da classe Two Jobs é descrito pela inequação

$$
\left(p_{i}+r_{i}-r_{j}\right) s_{i}+\left(p_{j}+r_{j}-r_{i}\right) s_{j} \geq p_{i} p_{j}+r_{i} p_{j}+r_{j} p_{i}
$$

que é válida para o JS.

Demonstração: Uma vez que o head representa o instante de tempo em que a operação está disponível, então para toda operação $i$, temos que

$$
s_{i} \geq r_{i}
$$

Além disso, se a operação $i$ precede a operação $j$, então

$$
s_{j} \geq\left(s_{i}+p_{i}\right) \geq\left(r_{i}+p_{i}\right)
$$

Considerando $\alpha$ e $\beta$ dois números positivos reais, a partir de (3.2) e (3.3), temos

$$
\alpha s_{i}+\beta s_{j} \geq \alpha r_{i}+\beta\left(r_{i}+p_{i}\right)
$$

Por outro lado, se $j$ precede $i$, então

$$
s_{i} \geq\left(s_{j}+p_{j}\right) \geq\left(r_{j}+p_{j}\right)
$$

e através de $(3.5)$ e de $s_{j} \geq r_{j}$, temos

$$
\alpha s_{i}+\beta s_{j} \geq \alpha\left(r_{j}+p_{j}\right)+\beta r_{j}
$$

Como não sabemos a ordem de execução entre as operações $i$ e $j$, as inequações (3.4) e (3.6) são ambas verdadeiras se os termos do lado direito coincidirem, isto é:

$$
\begin{aligned}
\alpha r_{i}+\beta\left(r_{i}+p_{i}\right) & =\alpha\left(r_{j}+p_{j}\right)+\beta r_{j} \Rightarrow \\
\beta\left(r_{i}+p_{i}-r_{j}\right) & =\alpha\left(r_{j}+p_{j}-r_{i}\right) .
\end{aligned}
$$

A igualdade em (3.7) é válida se $\alpha=r_{i}+p_{i}-r_{j}$ e $\beta=r_{j}+p_{j}-r_{i}$. Como por hipótese $\alpha, \beta>0, \log r_{i}+p_{i}>r_{j}$ e $r_{j}+p_{j}>r_{i}$. Por fim, substituindo os valores de $\alpha$ e $\beta$ nas inequações (3.4) ou (3.6), temos a inequação (3.1).

A classe de inequação Two Jobs foi originalmente proposta por Balas (1985). Neste trabalho, Balas apresenta um estudo poliédrico para o JS e a inequação válida Two Jobs é desenvolvida quando as operações são analisadas conforme a máquina habilitada a processálas.

Propriedade 2 (Reverse Two Jobs). Sejam $i$ e $j$ duas operações tais que $m_{i}=m_{j}$. Se $q_{i}+p_{i}>q_{j}$ e $q_{j}+p_{j}>q_{i}$, então a inequação

$$
\left(p_{i}+q_{i}-q_{j}\right)\left(C_{\text {Max }}-s_{i}\right)+\left(p_{j}+q_{j}-q_{i}\right)\left(C_{\text {Max }}-s_{i}\right) \geq p_{i}^{2}+p_{i} p_{j}+p_{j}^{2}+p_{i} q_{i}+p_{j} q_{j}
$$

é válida para o JS. 
Demonstração: Como $C_{\text {Max }}$ é um limitante superior para instante de término para qualquer operação, então $C_{M a x} \geq s_{i}+p_{i}+q_{i}$ e $C_{M a x} \geq s_{j}+p_{j}+q_{j}$. Sejam $\alpha$ e $\beta$ dois números reais positivos. Se a operação $i$ precede a operação $j$, então

$$
\begin{array}{r}
s_{j} \geq s_{i}+p_{i} \Rightarrow-s_{i} \geq-s_{j}+p_{i} \Rightarrow \\
\left(C_{\text {Max }}-s_{i}\right) \geq C_{\text {Max }}-s_{j}+p_{i} \geq p_{j}+q_{j}+p_{i} .
\end{array}
$$

Assim,

$$
\alpha\left(C_{\text {Max }}-s_{i}\right)+\beta\left(C_{\text {Max }}-s_{j}\right) \geq \alpha\left(p_{j}+q_{j}+p_{i}\right)+\beta\left(p_{j}+q_{j}\right) .
$$

Agora, se $j$ precede $i$, então

$$
\begin{aligned}
s_{i} & \geq s_{j}+p_{j} \Rightarrow-s_{j} \geq-s_{i}+p_{j} \Rightarrow \\
\left(C_{\text {Max }}-s_{j}\right) & \geq C_{\text {Max }}-s_{i}+p_{j} \geq p_{i}+q_{i}+p_{j}
\end{aligned}
$$

e, portanto,

$$
\alpha\left(C_{M a x}-s_{i}\right)+\beta\left(C_{M a x}-s_{j}\right) \geq \alpha\left(p_{i}+q_{i}\right)+\beta\left(p_{i}+q_{i}+p_{j}\right)
$$

Para que ambas as inequações (3.9) e (3.10) sejam verdadeiras, devemos ter que $\alpha=p_{i}+q_{i}-q_{j}$ e $\beta=p_{j}+q_{j}-q_{i}$. Como $\alpha, \beta>0$, então $p_{i}+q_{i}>q_{j}$ e $p_{j}+q_{j}>q_{i}$. Além disso, substituindo os valores $\alpha$ e $\beta$ em (3.9) ou (3.10), obtemos a inequação (3.8).

Propriedade 3 (Half Cuts). Para cada máquina $k \in M, S \subseteq \mathcal{O}_{k}$ e $i \in S$, a inequação

$$
s_{i} \geq \operatorname{Min}_{j \in S} r_{j}+\sum_{\substack{j \in S \\ j \neq i}} y_{j i} \cdot p_{j}
$$

denominada de Half Cuts é válida para o JS.

Demonstração: Suponha que a ordem de execução das operações em $S$ seja dada pelo vetor permutação de operações

$$
\pi=(\pi(1), \ldots, \pi(i), \ldots, \pi(|S|))
$$

em que $\pi(j) \in S, j=1, \ldots,|S|$. Assim, considerando a ordem de execução em $\pi$, temos

$$
\begin{aligned}
s_{\pi(i)} & \geq s_{\pi(1)}+p_{\pi(1)}+\ldots+p_{\pi(i-1)} \\
& =s_{\pi(1)}+p_{\pi(1)}+\ldots+p_{\pi(i-1)}+0 \cdot p_{\pi(i+1)}+\ldots+0 \cdot p_{\pi(n)} \\
& =s_{\pi(1)}+\sum_{\substack{j=1 \\
j \neq i}}^{|S|} y_{\pi(j) \pi(i)} \cdot p_{\pi(j)} .
\end{aligned}
$$

Como $s_{\pi(1)} \geq \operatorname{Min}_{j=1, \ldots,|S|} r_{\pi(j)}$, portanto

$$
s_{i} \geq \operatorname{Min}_{j \in S} r_{j}+\sum_{\substack{j \in S \\ j \neq i}} y_{j i} \cdot p_{j}
$$

e independe da ordenação das operações em $S$. 
Propriedade 4 (Reverse Half Cuts). Para cada máquina $k \in M, S \subseteq \mathcal{O}_{k}$ e $i \in S$, a inequação válida Reverse Half Cuts é definida por

$$
C_{M a x}-s_{i} \geq p_{i}+\operatorname{Min}_{j \in S} q_{j}+\sum_{\substack{j \in S \\ j \neq i}} y_{i j} \cdot p_{j} .
$$

Demonstração: Suponha novamente que a ordem de execução das operações em $S$ seja dada por $\pi=(\pi(1), \ldots, \pi(i), \ldots, \pi(|S|))$. Logo, conforme a ordenação em $\pi$, temos:

$$
\begin{aligned}
s_{\pi(|S|)} & \geq s_{\pi(i)}+p_{\pi(i)}+\ldots+p_{\pi(|S|-1)} \Rightarrow \\
-s_{\pi(|S|)} & \leq-s_{\pi(i)}-p_{\pi(i)}-\ldots-p_{\pi(|S|-1)} \Rightarrow \\
-s_{\pi(|S|)}-p_{\pi(|S|)} & \leq-s_{\pi(i)}-p_{\pi(i)}-\ldots-p_{\pi(|S|-1)}-p_{\pi(|S|)} \Rightarrow \\
-s_{\pi(i)}-p_{\pi(i)} & \geq-s_{\pi(|S|)}-p_{\pi(|S|)}+p_{\pi(i+1)}+\ldots+p_{\pi(|S|)} \\
& =-s_{\pi(|S|)}-p_{\pi(|S|)}+\sum_{\substack{j=1 \\
j \neq i}}^{|S|} y_{\pi(i) \pi(j)} \cdot p_{\pi(j)} .
\end{aligned}
$$

Para todo $j \in S, C_{M a x} \geq s_{j}+p_{j}+q_{j}$. Assim, adicionando $C_{\text {Max }}$ em ambos os lados de (3.13), temos:

$$
\begin{aligned}
C_{M a x}-s_{i}-p_{i} & \geq C_{M a x}-s_{\pi(|S|)}-p_{\pi(|S|)}+\sum_{\substack{j \in S \\
j \neq i}} y_{i j} \cdot p_{j} \\
& \geq q_{\pi(|S|)}+\sum_{\substack{j \in S \\
j \neq i}} y_{i j} \cdot p_{j} \\
& \geq \operatorname{Min}_{j \in S} q_{j}+\sum_{\substack{j \in S \\
j \neq i}} y_{i j} \cdot p_{j} .
\end{aligned}
$$

Portanto,

$$
C_{M a x}-s_{i} \geq p_{i}+\operatorname{Min}_{j \in S} q_{j}+\sum_{\substack{j \in S \\ j \neq i}} y_{i j} \cdot p_{j}
$$

Propriedade 5 (Late Job Cuts). Para cada máquina $k \in M$, sejam $S \subseteq \mathcal{O}_{k}, i \in S$ e $l \in \mathcal{O}_{k} . A$ inequação

$$
s_{i} \geq r_{l}+\sum_{\substack{j \in S \\ j \neq i}} y_{j i} \cdot p_{j}-\sum_{\substack{j \in S \\ j \neq l}} y_{j l}\left(r_{l}-r_{j}\right)^{+}
$$

é válida para o JS.

Demonstração: Observe inicialmente que o termo $-\sum_{\substack{j \in S \\ j \neq l}} y_{j l}\left(r_{l}-r_{j}\right)^{+}$da inequação (3.16) é sempre menor ou igual a zero e independe do sequenciamento das operações em $S$. Como, por hipótese, a operação $l \in \mathcal{O}_{k}$ e $S \subseteq \mathcal{O}_{k}$, vamos dividir a demonstração em dois casos: $l \notin S$ ou $l \in S$.

- Caso 1: Suponha que $l \notin S$ e que a operação $l$ seja executada antes de todas as tarefas de $S$. Seja

$$
\pi=(\pi(1), \ldots, \pi(i), \ldots, \pi(|S|))
$$


a ordem de execução das operações em $S$. Assim, $s_{\pi(1)} \geq s_{l} \geq r_{l}$ e $y_{\pi(j) l}=0$ para todo $j=1, \ldots,|S|$. Portanto,

$$
\begin{aligned}
s_{\pi(i)} & \geq r_{\pi(1)}+p_{\pi(1)}+\ldots+p_{\pi(i-1)} \\
\geq & r_{l}+p_{\pi(1)}+\ldots+p_{\pi(i-1)} \\
& =r_{l}+\sum_{\substack{j=1 \\
j \neq i}}^{|S|} y_{\pi(j) \pi(i)} \cdot p_{\pi(j)} \\
& =r_{l}+\sum_{\substack{j=1 \\
j \neq i}}^{|S|} y_{\pi(j) \pi(i)} \cdot p_{\pi(j)}-\sum_{j=1}^{|S|} y_{\pi(j) l} \cdot\left(r_{l}-r_{\pi(j)}\right)^{+} .
\end{aligned}
$$

Suponha agora que operação $l$ seja processada após todas as operações de $S\left(y_{\pi(j) l}=1\right.$, para todo $j=1, \ldots,|S|)$. Se $r_{l} \leq r_{\pi(1)}$ então $\left(r_{l}-r_{\pi(1)}\right)^{+}=0$ e

$$
\begin{aligned}
s_{\pi(i)} & \geq r_{\pi}(i)+\sum_{\substack{j=1 \\
j \neq i}}^{|S|} y_{\pi(j) \pi(i)} \cdot p_{\pi(j)} \\
& \geq r_{l}+\sum_{\substack{j=1 \\
j \neq i}}^{|S|} y_{\pi(j) \pi(i)} \cdot p_{\pi(j)} \\
& =r_{l}+\sum_{\substack{j=1 \\
j \neq i}}^{|S|} y_{\pi(j) \pi(i)} \cdot p_{\pi(j)}-y_{\pi(1) l} \cdot\left(r_{l}-r_{\pi(1)}\right)^{+} \\
& \geq r_{l}+\sum_{\substack{|S| \\
j=1 \\
j \neq i}} y_{\pi(j) \pi(i)} \cdot p_{\pi(j)}-\sum_{j=1}^{|S|} y_{\pi(j) l} \cdot\left(r_{l}-r_{\pi(j)}\right)^{+} .
\end{aligned}
$$

Se $r_{l} \geq r_{\pi(1)}$ então $r_{\pi(1)}=r_{l}-r_{l}+r_{\pi(1)}=r_{l}-y_{\pi(1) l} \cdot\left(r_{l}-r_{\pi(1)}\right)^{+}$. Logo,

$$
\begin{aligned}
s_{\pi(i)} & \geq r_{\pi(1)}+p_{\pi(1)}+\ldots+p_{\pi(i-1)} \\
& =r_{l}+\sum_{\substack{j=1 \\
j \neq i}}^{|S|} y_{\pi(j) \pi(i)} \cdot p_{\pi(j)}-y_{\pi(1) l} \cdot\left(r_{l}-r_{\pi(1)}\right)^{+} \\
& \geq r_{l}+\sum_{\substack{j=1 \\
j \neq i}}^{|S|} y_{\pi(j) \pi(i)} \cdot p_{\pi(j)}-\sum_{j=1}^{|S|} y_{\pi(j) l} \cdot\left(r_{l}-r_{\pi(j)}\right)^{+} .
\end{aligned}
$$

- Caso 2: Considere $l \in S$ e que a ordem de processamento das tarefas de $S$ seja dada por $\pi=(\pi(1), \ldots, \pi(m), l, \pi(m+1), \ldots, \pi(i), \ldots, \pi(|S|))$. Se $r_{\pi(1)} \geq r_{l}$, então 


$$
\begin{aligned}
s_{\pi(i)} & \geq r_{\pi(1)}+p_{\pi(1)} \ldots+p_{\pi(m)}+p_{l}+p_{\pi(m+1)}+\ldots+p_{\pi(i-1)} \\
& \geq r_{l}+p_{\pi(1)} \ldots+p_{\pi(m)}+p_{l}+p_{\pi(m+1)}+\ldots+p_{\pi(i-1)} \\
& =r_{l}+\sum_{\substack{j \in S \\
j \neq \pi(i)}} y_{j \pi(i)} \cdot p_{j} \\
& \geq r_{l}+\sum_{\substack{j \in S \\
j \neq \pi(i)}} y_{j \pi(i)} \cdot p_{j}-\sum_{\substack{j \in S \\
j \neq l}} y_{j l} \cdot\left(r_{l}-r_{j}\right)^{+} .
\end{aligned}
$$

Por fim, se $r_{l} \geq r_{\pi(1)}$, assim como no caso 1 , temos que $r_{\pi(1)}=r_{l}-y_{\pi(1) l} \cdot\left(r_{l}-r_{\pi(1)}\right)^{+}$ e, logo,

$$
s_{\pi(i)} \geq r_{l}+\sum_{\substack{j \in S \\ j \neq \pi(i)}} y_{j \pi(i)} \cdot p_{j}-\sum_{\substack{j \in S \\ j \neq l}} y_{j l} \cdot\left(r_{l}-r_{j}\right)^{+}
$$

Portanto, de acordo com os resultados obtidos em todas as situações, podemos concluir que a inequação (3.16) é válida.

Embora não tenha sido intitulada por Late Jobs Cuts, tais inequações foram originalmente propostas por Dyer e Wolsey (1990) para o problema de programação de tarefas em uma única máquina com heads e tails.

Propriedade 6 (Reverse Late Job Cuts). Para cada máquina $k \in M$, sejam $S \subseteq \mathcal{O}_{k}$, $i \in S$ e $l \in \mathcal{O}_{k}$. A inequação

$$
C_{M a x}-s_{i} \geq p_{i}+q_{l}+\sum_{\substack{j \in S \\ j \neq i}} y_{i j} \cdot p_{j}-\sum_{\substack{j \in S \\ j \neq l}} y_{l j} \cdot\left(q_{l}-q_{j}\right)^{+}
$$

é denominada de Reverse Late Job Cuts e representa uma inequação válida para o JS.

Demonstração: Assim como na propriedade 5, vamos dividir esta demonstração em dois casos: $l \notin S$ e $l \in S$.

- Caso 1: Suponha que a ordem de processamento das operações em $S$ seja dada por $\pi=(\pi(1), \ldots, \pi(i), \ldots, \pi(|S|))$ e $l \notin S$. Da inequação (3.14), temos que

$$
C_{M a x}-s_{\pi(i)}-p_{\pi(i)} \geq C_{M a x}-s_{\pi(|S|)}-p_{\pi(|S|)}+\sum_{\substack{j=1 \\ j \neq i}}^{|S|} y_{\pi(i) \pi(j)} \cdot p_{\pi(j)}
$$

Se $y_{\pi(|S|) l}=1$, então $s_{l} \geq s_{\pi(|S|)}+p_{\pi(|S|)}$ e $y_{l \pi(j)}=0$ para todo $j=1, \ldots,|S|$. Como $C_{M a x} \geq s_{l}+p_{l}+q_{l}, \log \mathrm{O}$ 


$$
\begin{aligned}
C_{M a x}-s_{\pi(i)}-p_{\pi(i)} & \geq C_{M a x}-s_{\pi(|S|)}-p_{\pi(|S|)}+\sum_{\substack{j=1 \\
j \neq i}}^{|S|} y_{\pi(i) \pi(j)} \cdot p_{\pi(j)} \\
& \geq C_{M a x}-s_{l}+\sum_{\substack{j=1 \\
j \neq i}}^{|S|} y_{\pi(i) \pi(j)} \cdot p_{\pi(j)} \\
& \geq q_{l}+p_{l}+\sum_{\substack{j=1 \\
j \neq i}}^{|S|} y_{\pi(i) \pi(j)} \cdot p_{\pi(j)} \\
& \geq q_{l}+\sum_{\substack{j=1 \\
j \neq i}}^{|S|} y_{\pi(i) \pi(j)} \cdot p_{\pi(j)} \\
& =q_{l}+\sum_{\substack{j=1 \\
j \neq i}} y_{\pi(i) \pi(j)} \cdot p_{\pi(j)}-\sum_{j=1}^{|S|} y_{l \pi(j)} \cdot\left(q_{l}-q_{\pi(j)}\right)^{+}
\end{aligned}
$$

Portanto,

$$
C_{M a x}-s_{i} \geq p_{i}+q_{l}+\sum_{\substack{j \in S \\ j \neq i}} y_{i j} \cdot p_{j}-\sum_{j \in S} y_{l j} \cdot\left(q_{l}-q_{j}\right)^{+} .
$$

Agora, considere que a situação em operação $l$ seja executada antes de todas as operações de $S$. Conforme a inequação (3.15), temos que

$$
C_{M a x}-s_{\pi(i)} \geq p_{\pi(i)}+q_{\pi(|S|)}+\sum_{\substack{j \in S \\ j \neq i}} y_{\pi(i) \pi(j)} \cdot p_{\pi(j)}
$$

Se $q_{\pi(|S|)} \geq q_{l}$, então

$$
\begin{aligned}
C_{M a x}-s_{\pi(i)} & \geq p_{\pi(i)}+q_{\pi(|S|)}+\sum_{\substack{j \in S \\
j \neq i}} y_{\pi(i) \pi(j)} \cdot p_{\pi(j)}+ \\
& \geq p_{\pi(i)}+q_{l}+\sum_{\substack{j=1 \\
j \neq i}}^{|S|} y_{\pi(i) \pi(j)} \cdot p_{\pi(j)} \\
& =p_{\pi(i)}+q_{l}+\sum_{\substack{j=1 \\
j \neq i}}^{|S|} y_{\pi(i) \pi(j)} \cdot p_{\pi(j)}-y_{l \pi(|S|)}\left(q_{l}-q_{\pi(|S|}\right)^{+} \\
& \geq p_{\pi(i)}+q_{l}+\sum_{\substack{j=1 \\
j \neq i}}^{|S|} y_{\pi(i) \pi(j)} \cdot p_{\pi(j)}-\sum_{j=1}^{|S|} y_{l \pi(j)} \cdot\left(q_{l}-q_{\pi(j)}\right)^{+},
\end{aligned}
$$


mas se $q_{l} \geq q_{\pi(|S|)}$, temos que $q_{\pi(|S|)}=q_{l}-y_{l \pi(|S|)} \cdot\left(q_{l}-q_{\pi(|S|)}\right)$. Logo,

$$
\begin{aligned}
C_{M a x}-s_{\pi(i)} & \geq p_{\pi(i)}+q_{\pi(|S|)}+\sum_{\substack{j=1 \\
j \neq i}}^{|S|} y_{\pi(i) \pi(j)} \cdot p_{\pi(j)} \\
& =p_{\pi(i)}+q_{l}+\sum_{\substack{j=1 \\
j \neq i}}^{|S|} y_{\pi(i) \pi(j)} \cdot p_{\pi(j)}-y_{l \pi(|S|)}\left(q_{l}-q_{\pi(|S|}\right)^{+} \\
& \geq p_{\pi(i)}+q_{l}+\sum_{\substack{j=1 \\
j \neq i}}^{|S|} y_{\pi(i) \pi(j)} \cdot p_{\pi(j)}-\sum_{j=1}^{|S|} y_{l \pi(j)} \cdot\left(q_{l}-q_{\pi(j)}\right)^{+} .
\end{aligned}
$$

- Caso 2: Seja $l \in S$ e $\pi=(\pi(1), \ldots, \pi(i), \ldots, \pi(m), l, \pi(m+1) \ldots, \pi(|S|))$ ordem de processamento das operações em $S$. Se $q_{\pi(|S|)} \geq q_{l}$, então, a partir da inequação (3.19), temos

$$
C_{M a x}-s_{\pi(i)} \geq p_{\pi(i)}+q_{l}+\sum_{\substack{j \in S \\ j \neq \pi(i)}} y_{\pi(i) j} \cdot p_{j}-\sum_{\substack{j \in S \\ j \neq l}} y_{l j} \cdot\left(q_{l}-q_{j}\right)^{+} .
$$

Caso $q_{l} \geq q_{\pi(|S|)}$, então

$$
\begin{aligned}
C_{M a x}-s_{\pi(i)} & \geq p_{\pi(i)}+q_{l}+\sum_{\substack{j \in S \\
j \neq \pi(i)}} y_{\pi(i) j} \cdot p_{j}-y_{l \pi(|S|)}\left(q_{l}-q_{\pi(|S|}\right)^{+} \\
& \geq p_{\pi(i)}+q_{l}+\sum_{\substack{j \in S \\
j \neq \pi(i)}} y_{\pi(i) j} \cdot p_{\pi(j)}-\sum_{\substack{j \in S \\
j \neq l}} y_{l j} \cdot\left(q_{l}-q_{j}\right)^{+} .
\end{aligned}
$$

Portanto, a inequação (3.17) é válida.

\subsection{Inequações válidas para job-shop flexível}

Nesta seção, apresentamos várias famílias de desigualdades válidas para o JSF e que serão incorporadas a um algoritmo de $\mathrm{PC}$ e $B \& C$. Dividimos as classes de desigualdades válidas em dois conjuntos. O primeiro conjunto é obtido a partir de algumas famílias de inequações válidas para o problema de Ordenação Linear (Linear Ordering Problem) e o segundo conjunto através da reformulação de algumas famílias de inequações para o JS.

O Problema de Ordenação Linear (POL) é um problema NP-difícil (KARP, 1972) e foi inicialmente proposto por Chenery e Watanabe (1958). O POL possui um grande número de aplicações em diversos campos, tais como a triangularização de matrizes de entrada e saída (input-output matrices) e atividade de estratificação em arqueologia. A primeira aplicação permite que economistas extraiam algumas informações sobre a estabilidade da economia e a segunda é utilizada para encontrar a ordem cronológica mais provável de amostras disponíveis em diferentes locais. O livro Martí e Reinelt (2011) apresenta outras aplicações e diversas técnicas de resolução para o POL.

Para descrever o problema, considere o digrafo $D=(V, A)$, em que $V$ representa um conjunto de $n$ vértice e $A \subseteq V \times V$ um conjunto de arcos. Seja $c_{i j}$ o custo de cada arco $(i, j)$ em $D$. Um digrafo é denominado de completo, e denotado por $D_{n}=\left(V \cdot A_{n}\right)$, se para todo 
par $i$ e $j$ de vértices, $D$ contém os $\operatorname{arcos}(i, j)$ e $(j, i)$. Um torneio (tournament) é um digrafo tal que para quaisquer vértices $i$ e $j$, existe um único arco entre eles: ou $(i, j)$ ou $(j, i)$. Dessa forma, um torneio torneio é um grafo orientado obtido ao se escolher uma direção para cada aresta em um grafo completo não-direcionado.

Um conjunto de $\operatorname{arcos} P=\left\{\left(v_{1}, v_{2}\right),\left(v_{2}, v_{3}\right), \ldots,\left(v_{k-1}, v_{k}\right)\right\}$ em $D=(V, A)$, tal que $v_{i} \neq v_{j}$ para todo $i \neq j$, é denominado de caminho de comprimento $k-1$. Além disso, se $\left(v_{k}, v_{1}\right) \in A$, então $C=P \cup\left\{\left(v_{k}, v_{1}\right)\right\}$ recebe o nome de $k$-diciclo.

Uma ordenação linear, ou permutação, de um conjunto de vértices $V$ de um digrafo $D$ é definida por uma função bijetiva $\pi:\{1,2, \ldots, n\} \rightarrow V$, tal que $\pi(k)=v$ denota que $v$ ocupa $k$-ésima posição em $\pi$. Para $i, j \in V$, dizemos que $i$ está antes de $j$ se $\pi^{-1}(i)<\pi^{-1}(j)$.

O custo total associado a uma ordenação linear $\pi$ é dado por

$$
\sum_{\substack{i, j \in V \\ \pi^{-1}(i)<\pi^{-1}(j)}} c_{i j} .
$$

Assim, dado um digrafo $D=(V, A)$, o POL consiste em encontrar uma permutação dos vértices que maximize o seu custo total.

Dada uma ordenação linear de um conjunto de vértices $V$ de um digrafo, o conjunto de $\operatorname{arcos}\left\{(i, j) ; \pi^{-1}(i)<\pi^{-1}(j)\right\}$ define um torneio acíclico em $V$ e, se $(V, T)$ é um torneio acíclico, então $(V, T)$ induz uma ordenação linear em $V$. Dessa forma, o POL equivale a encontrar um torneio acíclico $(V, T)$ em $D_{n}$ tal que $c(T)=\sum_{(i, j) \in T} c_{i j}$ seja o maior possível.

Para o JSF, uma vez definido o conjunto de operações que serão processadas em cada máquina $k \in M$, o problema consiste em definir a ordem de processamento dessas operações, de tal modo que o tempo de conclusão da última tarefa seja o menor possível. Determinar um sequenciamento entre essas operações equivale a determinar um torneio acíclico em um grafo completo. Dessa forma, o subproblema de sequenciamento do JSF pode ser modelo pelo POL.

Para definir o conjunto dos torneios acíclicos associado à máquina $k$, considere $V \subseteq \mathcal{O}_{k}$ como sendo o conjunto de operações atribuídas à máquina $k$, isto é, $V=\left\{i \in O ; x_{i k}=1\right\}$. Seja $D_{n}=\left(V, A_{n}\right)$ o digrafo completo de ordem $n$, em que $n=|V|$ e $A_{n}$ é o conjunto de $\operatorname{arcos}$ da forma $(i, j)$ e $(j, i)$, para todo $i, j \in V$. Definimos o conjunto de todos os torneios acíclicos associados ao digrafo completo $D_{n}$ por

$$
\mathscr{T}_{n}=\left\{T \subseteq A_{n} ;(V, T) \text { é um torneio acíclico }\right\} .
$$

Grötschel, Jünger e Reinelt (1984) e Grötschel, Jünger e Reinelt (1985) descrevem um estudo poliédrico para o POL. Nesses trabalhos, os autores estudam o poliedro relacionado com $\mathscr{T}_{n}$ e um conjunto de facetas e inequações válidas para ele. Os autores apresentam um algoritmo de PC para o POL.

Na formulação poliédrica de $\mathscr{T}_{n}$ proposta por Grötschel, Jünger e Reinelt (1984), um vetor é utilizado para representar cada torneio acíclico. Para isso, considere $m=\left|A_{n}\right|=$ $n(n-1)$ e o vetor $y \in \mathbb{R}^{m}$, tal que cada componente de $y$ é indexado por um arco $(i, j) \in A_{n}$. Para qualquer conjunto de arcos $A \subseteq A_{n}$, os autores definem o vetor de incidência $y^{A} \in \mathbb{R}$ de $A$ por

$$
\begin{cases}y_{i j}^{A}=1, & \text { se }(i, j) \in A \\ y_{i j}^{A}=0, & \text { se }(i, j) \notin A .\end{cases}
$$


O envoltório convexo dos vetores de incidência de todos os torneios em $D_{n}$ é o politopo $P^{n}$ determinado por

$$
P^{n}=\operatorname{conv}\left\{y^{T} \in \mathbb{R}^{m} ; T \in \mathscr{T}_{n}\right\} .
$$

Dessa forma, como todo vértice que $P^{n}$ corresponde a um torneio acíclico, o POL pode ser resolvido através do $\mathrm{PL}$

\section{Maximizar cy \\ Sujeito a $y \in P^{n}$.}

Embora, em tese, o POL possa ser resolvido através de LP, a resolução do problema é viável somente se o conjunto $P^{n}$ puder ser representado por um conjunto de equações e inequações lineares. Como o POL é um problema NP-difícil, pode não ser possível encontrar uma descrição completa do conjunto $P^{n}$. Entretanto, Grötschel, Jünger e Reinelt (1985) foram capazes de encontrar um conjunto de equações e inequações que descrevem parcialmente o conjunto $P^{n}$. Na sequência, vamos descrever duas inequações que representam facetas de $P^{n}$ e que serão utilizadas neste trabalho.

$\mathrm{O}$ vetor de incidência de um torneio $T$ define uma ordem entre as operações que são as extremidades dos arcos de $T$. Isso é equivalente à variável binária $y$ utilizada na formulação MILP-1. Portanto, a variável $y^{T}$ utilizada na definição de $P^{n}$ pode ser substituída pela variável binária $y$ utilizada no modelo MILP-1.

Dada a máquina $k \in M$, inicialmente definimos o conjunto $V$ de vértices em $D_{n}$ como sendo o conjunto de operações que são atribuídas a $k$. Podemos estender a definição de $V$ para qualquer conjunto de operações em $V \subseteq \mathcal{O}_{k}$, pois caso uma operação $i \in S$ não seja atribuída à máquina $k$, as variáveis binárias $y$ que possuem como índice os termos $i$ e $k$ são todas nulas e, portanto, todo torneio acíclico $(V, T)$ não passará pelas operações que não foram atribuídas a $k$.

Propriedade 7 (3-Diciclo). Dado $k \in M$, considere o digrafo completo $D_{n}=\left(V, A_{n}\right)$, em que $V \subseteq \mathcal{O}_{k}$. Para todo diciclo $C \subseteq A_{n}$ de tamanho 3, a inequação

$$
\sum_{(i, j) \in A} y_{i j k} \leq 2
$$

define uma faceta para $P^{n}$.

A inequação (3.22) diz que, se a operação $i_{1}$ é sequenciada antes de $i_{2}$ e $i_{2}$ é sequenciada antes de $i_{3}$, então $i_{1}$ deve ser sequenciada após $i_{3}$. A Figura 9 apresenta 3 -diciclo.

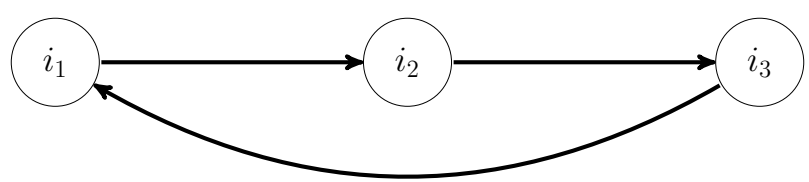

Figura 9 - Exemplo do Grafo 3-Diciclo.

Propriedade 8 (א-Fence). Sejam $k \in M, V \subseteq \mathcal{O}_{k}$ e $D_{n}=\left(V, A_{n}\right)$, o digrafo completo de ordem $n \geq 6$. Para todo inteiro $\kappa \geq 3$, o grafo $F=\left(V_{F}, A_{F}\right)$ é denominado de $\kappa$-Fence se 
$V_{F}$ é a união dos conjuntos disjuntos $U=\left\{i_{1}, i_{2}, \ldots, i_{k}\right\} \subseteq V$ e $W=\left\{j_{1}, j_{2}, \ldots, j_{k}\right\} \subseteq V$ e $A_{F}$ é definido pela união dos arcos da forma $\left\{\left(i_{l}, j_{l}\right) ; l=1, \ldots, k\right\}$ e $\left\{\left(j_{l}, i_{m}\right) ; l, m \in\right.$ $\{1, \ldots, k\}, l \neq m\}$. Os arcos da forma $\left(i_{l}, j_{l}\right)$ são denominados de estacas (pales) e os da forma $\left(j_{l}, i_{m}\right)$ de piquetes (picket). A inequação definida por

$$
\sum_{(i, j) \in A} y_{i j k} \leq \kappa^{2}-\kappa+1
$$

é denominada de inequação K-Fence e define uma faceta para $P^{n}$.

A Figura 10 apresenta um grafo 3-Fence.

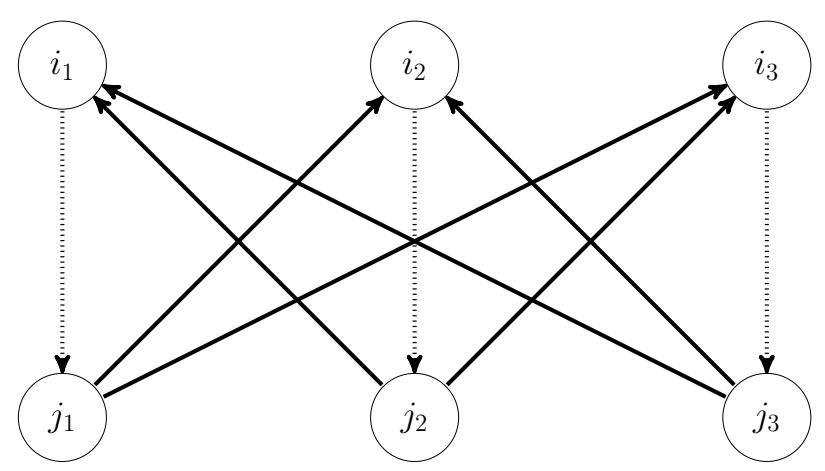

Figura 10 - Exemplo do Grafo 3-Fence. As linhas pontilhadas representam os arcos estacas e as linhas contínuas os arcos piquetes.

Omitiremos as demonstrações das propriedades 7 e 8. As demonstrações e a descrição de outras facetas triviais do conjunto $P^{n}$ podem ser encontradas na pesquisa de Grötschel, Jünger e Reinelt (1985).

O segundo conjunto de classes de inequações válidas utilizado neste trabalho é uma extensão de algumas das inequações válidas utilizadas por Applegate e Cook (1991) para o JS.

Assim como no JS, o head $r_{i}$ de uma operação $i \in O$ representa o menor tempo possível para o início de seu processamento e o tail $q_{i}$ representa o menor tempo possível para finalizar a tarefa $j$, após a execução da operação $i \in O$. Considere $f_{t}, l_{t} \in O$ como sendo a primeira e última operação, respectivamente, de cada tarefa $t \in T$. Como não é possível definir previamente em que máquina uma operação $i \in O$ será executada em cada tarefa, definimos $r_{i}$ por:

$$
\left\{\begin{array}{l}
r_{i}=0, \text { se } i=f_{t} \\
r_{i}=r_{i-1}+\operatorname{Min}_{k \in \mathscr{M}_{i-1}} p_{i-1, k}, \quad i=\left(f_{t}+1\right), \ldots, l_{t} .
\end{array}\right.
$$

De forma análoga, o tail da operação $i \in O$ é definido por:

$$
\left\{\begin{array}{l}
q_{i}=0, \text { se } i=l_{t} \\
q_{i}=q_{i+1}+\operatorname{Min}_{k \in \mathscr{M}_{i+1}} p_{i+1, k}, \quad i=1, \ldots,\left(l_{t}-1\right) .
\end{array}\right.
$$

Como as inequações válidas são definidas para cada máquina $k \in M$, considere $S \subseteq \mathcal{O}_{k}$ como sendo o subconjunto de operações que podem ser processadas na máquina $k$. Sejam $r_{S}=\operatorname{Min}_{i \in S} r_{i}, q_{S}=\operatorname{Min}_{i \in S} q_{i}$. Ademais, para facilitar a notação, vamos definir uma variável 
binária para identificar explicitamente se a operação $i \in S$ precede ou não a operação $j \in S$ em $k$. Para isso, a variável $y_{i j}$ do modelo MILP-1 é substituída pela variável $y_{i j k}$ e esta recebe o valor 1 se a operação $i$ precede a operação $j$ na máquina $k$ e 0 , se $i$ não precede $j$, ou $x_{i k}=0$ ou $x_{j k}=0$. As inequações serão descritas na sequência.

Propriedade 9 (Two Jobs). Sejam $i, j \in \mathcal{O}_{k}$ e suponha que $r_{i}+p_{i k}>r_{j}$ e $r_{j}+p_{j k}>r_{i}$. A inequação Two-Job Cuts é definida por

$$
\begin{aligned}
& \left(r_{i}+p_{i k}-r_{j}\right) \cdot s_{i} \cdot\left(y_{i j k}+y_{j i k}\right)+\left(r_{j}+p_{j k}-r_{i}\right) \cdot s_{j} \cdot\left(y_{i j k}+y_{j i k}\right) \geq \\
& \left(r_{i}+p_{i k}-r_{j}\right) \cdot r_{i} \cdot\left(y_{i j k}+y_{j i k}\right)+\left(r_{j}+p_{j k}-r_{i}\right) \cdot\left(r_{i}+p_{i k}\right) \cdot\left(y_{i j k}+y_{j i k}\right)
\end{aligned}
$$

e é válida para o JSF.

Demonstração: Para verificar que a inequação (3.26) é válida, observe inicialmente que, se as operações $i$ e $j$ forem atribuídas à mesma máquina $k\left(x_{i k}=x_{j k}=1\right)$, então $y_{i j k}+y_{j i k}=1$. Assim,

$$
\begin{aligned}
& \left(r_{i}+p_{i k}-r_{j}\right) \cdot s_{i} \cdot\left(y_{i j k}+y_{j i k}\right)+\left(r_{j}+p_{j k}-r_{i}\right) \cdot s_{j} \cdot\left(y_{i j k}+y_{j i k}\right)= \\
& \left(r_{i}+p_{i k}-r_{j}\right) \cdot s_{i}+\left(r_{j}+p_{j k}-r_{i}\right) \cdot s_{j} \geq \\
& \left(r_{i}+p_{i k}-r_{j}\right) \cdot r_{i}+\left(r_{j}+p_{j k}-r_{i}\right) \cdot\left(r_{i}+p_{i k}\right)=p_{i k} \cdot p_{j k}+r_{i} \cdot p_{j k}+r_{j} \cdot p_{i k} .
\end{aligned}
$$

Portanto, a inequação Two Jobs equivale ao caso particular do JS (inequação (3.1)). Se $x_{i k}=0$ ou $x_{j k}=0$, então $y_{i j k}+y_{j i k}=0$, e neste caso, a inequação (3.26) é equivalente à restrição redundante $0 \geq 0$.

Assim como no caso do JS, para cada classe de inequação válida, associamos uma classe revertida que consiste no sequenciamento das operações em sua ordem inversa.

Propriedade 10 (Reverse Two Jobs). Considere $i$ e $j$ duas operações que podem ser executadas na máquina $k$ e suponha que $q_{i}+p_{i k}>q_{j}$ e $q_{j}+p_{j k}>q_{i}$. A inequação

$$
\begin{aligned}
& \left(q_{i}+p_{i k}-q_{j}\right) \cdot\left(C_{M a x}-s_{i}\right) \cdot\left(y_{i j k}+y_{j i k}\right)+\left(q_{j}+p_{j k}-q_{i}\right) \cdot\left(C_{M a x}-s_{j}\right) \cdot\left(y_{i j k}+y_{j i k}\right) \geq \\
& \left(q_{i}+p_{i k}-q_{j}\right) \cdot\left(q_{j}+p_{j k}+p_{i k}\right) \cdot\left(y_{i j k}+y_{j i k}\right)+\left(q_{j}+p_{j k}-q_{i}\right) \cdot\left(q_{j}+p_{j k}\right) \cdot\left(y_{i j k}+y_{j i k}\right) .
\end{aligned}
$$

é válida para o JSF.

Demonstração: Se as operações $i$ e $j$ são designadas à máquina $k$, então $y_{i j k}+y_{j i k}=1$. Assim, substituindo o valor de $y_{i j k}+y_{j i k}$ nos lados esquerdo e direito da inequação (3.27), a inequação se reduz a (3.8). Agora, se pelo menos uma das operações não for atribuída a máquina $k$, então $y_{i j k}+y_{j i k}=0$ e a inequação (3.27) se reduz à restrição $0 \geq 0$. Portanto, a inequação Reverse Two Jobs é válida.

A inequação (3.26) contém 4 termos não lineares $\left(s_{i} \cdot y_{i j k}, s_{i} \cdot y_{j i k}, s_{j} \cdot y_{i j k}\right.$ e $\left.s_{j} \cdot y_{j i k}\right)$ e a inequação (3.27) contém 6 termos não lineares $\left(C_{M a x} \cdot y_{i j k}, C_{M a x} \cdot y_{j i k}\right.$ e os 4 termos não lineares de (3.26)). Como cada termo é definido pelo produto de uma variável contínua por uma variável binária, este pode ser linearizado através do seguinte processo. Suponha que a variável contínua $c$ seja limitada por $0 \leq c \leq M$ e que $b$ seja a variável binária. A substituição do produto $c \cdot b$ por uma variável $z$ pode ser realizada desde que:

$$
\begin{aligned}
& z \leq M \cdot b \\
& z \leq c \\
& z \geq c-M(1-b) \\
& z \geq 0
\end{aligned}
$$


A identidade $c \cdot b=z$ é válida, pois se $b=0$, temos que $z=0$. Agora, se $b=1$, então por (3.30) temos que $z \geq c$, que combinado com (3.29), implica em $z=c$.

No processo de linearização, podemos limitar superiormente as variáveis contínuas $s_{i}$, $s_{l}$ e $C_{\text {Max }}$ pela constante $L$ utilizada no modelo MILP-1. Para outras técnicas de modelagem e situações de linearização, destacamos o livro de Williams (2013).

Propriedade 11 (Half Cuts). Para cada máquina $k, S \subseteq \mathcal{O}_{k}$ e $i \in S$,

$$
s_{i} \geq r_{S} \cdot x_{i k}+\sum_{\substack{j \in S \\ j \neq i}} y_{j i k} \cdot p_{j k}
$$

é válida para o JSF para qualquer sequenciamento de tarefas em $k$.

Demonstração: Seja $i^{\prime} \in S$ tal que $r_{i^{\prime}}=r_{S}$. Observe que se $x_{i k}=0$, então $y_{i j k}=0$ para todo $j \in S(j \neq i)$ e, portanto, $s_{i} \geq 0$. Se $x_{i k}=1$ e $x_{i^{\prime} k}=1$, então a inequação Half Cuts se reduz ao caso particular do JS (inequação (3.11)). Por fim, caso $x_{i^{\prime} k}=0$, a inequação (3.32) será dominada por uma outra inequação Half Cuts tal que o $\operatorname{Min}_{i \in S} r_{i}$ seja atribuído a alguma operação na máquina $k$.

Propriedade 12 (Reverse Half Cuts). Para cada máquina $k, S \subseteq \mathcal{O}_{k}$ e $i \in S$,

$$
C_{M a x}-s_{i} \geq p_{i} \cdot x_{i k}+q_{S} \cdot x_{i k}+\sum_{\substack{j \in S \\ j \neq i}} y_{i j k} \cdot p_{j k}
$$

é uma inequação válida para o JSF.

Demonstração: Se a operação $i \in S$ não é atribuída à máquina $k$, então (3.33) é reduzido a $C_{M a x}-s_{i} \geq 0$. Agora, se a operação $i$ é atribuída à máquina $k$, então a inequação Reverse Half Cuts equivale a $C_{M a x}-s_{i} \geq p_{i}+q_{S}+\sum_{\substack{j \in S \\ j \neq i}} y_{i j k} \cdot p_{j k}$. Assim, a última análise está relacionada com a operação $i^{\prime} \in S$ tal que $q_{i^{\prime}}=q_{S}$. Se $i^{\prime}$ for executada em $k$, então a inequação se reduz a inequação Reverse Half Cuts do JS (inequação (3.12)). Caso contrário, a inequação será dominada por outra inequação Reverse Half Cuts, tal que o $\operatorname{Min}_{i \in S} q_{i}$ seja atribuído a alguma operação na máquina $k$. Portanto, como em todas as situações, a inequação resultante é válida, e (3.33) também é válida.

Propriedade 13 (Late Job Cuts). Sejam $k \in M, S \subseteq \mathcal{O}_{k}, i \in S$ e $l \in \mathcal{O}_{k}$. A inequação

$$
s_{i} \geq r_{l} \cdot x_{i k} \cdot x_{l k}+\sum_{\substack{j \in S \\ j \neq i}} y_{j i k} \cdot p_{j k}-\sum_{\substack{j \in S \\ j \neq l}} y_{j l k}\left(r_{l}-r_{j}\right)^{+}
$$

é válida para o JSF.

Demonstração: Para verificar a validade do corte (3.34), vamos analisar as atribuições das operações $i$ e $l$ na máquina $k$. Se $x_{i k}=x_{l k}=0$, então $s_{i} \geq 0$. Se $x_{i k}=x_{l k}=1$, temos o caso particular do JS. Agora, se $x_{i k}=1$ e $x_{l k}=0$, temos que a inequação $s_{i} \geq \sum_{\substack{j \in S \\ j \neq i}} y_{j i k} \cdot p_{j k}$ é válida e é dominada pela inequação Half Cuts. Por fim, se $x_{i k}=0$ e $x_{l k}=1$, temos a inequação redundante $s_{i} \geq-\sum_{\substack{j \in S \\ j \neq l}} y_{j l k}\left(r_{l}-r_{j}\right)^{+} \geq 0$. 
Propriedade 14 (Reverse Late Job Cuts). Sejam $k \in M, S \subseteq \mathcal{O}_{k}, i \in S$ e $l \in \mathcal{O}_{k}$. A inequação

$$
C_{M a x}-s_{i} \geq p_{i} \cdot x_{i k}+q_{l} \cdot x_{i k} \cdot x_{l k}+\sum_{\substack{j \in S \\ j \neq i}} y_{i j k} \cdot p_{j k}-\sum_{\substack{j \in S \\ j \neq l}} y_{l j k}\left(q_{l}-q_{j}\right)^{+}
$$

é válida para o JSF.

Demonstração: Assim como na demonstração anterior, vamos verificar a validade das inequações resultantes a partir das atribuições das operações $i$ e $j$ em $k$. Se $x_{i k}=x_{l k}=0$, então $C_{M a x} \geq s_{i}$. Se $x_{i k}=1$ e $x_{l k}=0$, então temos a inequação $C_{M a x}-s_{i} \geq p_{i}+\sum_{\substack{j \in S \\ j \neq i}} y_{i j k} \cdot p_{j k}$, e esta, por sua vez, é dominada pela inequação Reverse Half Cuts. Agora, se $x_{i k}=0$ e $x_{l k}=1$, temos a inequação redundante $C_{M a x}-s_{i} \geq-\sum_{\substack{j \in S \\ j \neq l}} y_{l j k}\left(q_{l}-q_{j}\right)^{+}$. Por fim, se $x_{i k}=x_{l k}=1$, a inequação (3.35) equivale a inequação válida (3.17). Logo, como em todos casos, a inequação obtida é válida, assim (3.35) também é válida.

As inequações (3.34) e (3.35) são não-lineares pois contêm o termo $x_{i k} \cdot x_{l k}$ no lado direito da expressão. Entretanto, uma vez que $x_{i k} \cdot x_{l k} \geq x_{i k}+x_{l k}-1$, podemos obter uma inequação válida em que o termo é linearizado

\subsection{Reformulação do modelo MILP-1 (MILP-2)}

Para utilizar as inequações válidas no modelo MILP-1 em um método B\&C, foram realizadas duas alterações no modelo. A primeira alteração, já destacada na seção anterior, evidencia a máquina utilizada no processamento das operações através do aumento da dimensão da variável $y$. A segunda baseia-se na alteração da restrição (2.13). Na sequência, apresentamos as modificações realizadas e o modelo resultante.

A variável $y_{i j}$ do modelo MILP-1 é substituída pela variável $y_{i j k}$ e esta recebe o valor 1 se a operação $i$ precede a operação $j$ na máquina $k$ e 0 , se $i$ não precede $j$, ou $x_{i k}=0$ ou $x_{j k}=0$. Dessa forma, as restrições (2.13), (2.14) e (2.18) de MILP-1 são substituídas por:

$$
\begin{array}{ll}
y_{i j k}+y_{j i k} \geq x_{i k}+x_{j k}-1, & \forall k \in M, \forall(i, j) \in E_{k} \\
s_{i}+p_{i}-L \cdot\left(1-y_{i j k}\right) \leq s_{j}, & \forall k \in M, \forall(i, j) \in E_{k} \\
y_{i j k} \in\{0,1\}, & \forall k \in M, \forall(i, j) \in E_{k} .
\end{array}
$$

Entretanto, a solução ótima do modelo MILP-1 permite que a variável $y_{i j k}$ seja igual a 1 mesmo que $x_{i k}=0$ ou $x_{j k}=0$. Ou seja, se as operações $i, j \in O$ não são ambas atribuídas na máquina $k$ e desde que a restrição (3.37) não seja violada, podemos ter $y_{i j k}$ ou $y_{j i k}$ igual a 0 ou 1.

Para ilustrar o problema que poderá surgir, vamos analisar o comportamento da variável $y$ de uma solução factível para uma instância do JSF quando aplicada em uma inequação válida. Considere, como exemplo, a instância sfjs01 do conjunto de instâncias teste de Fattahi, Mehrabad e Jolai (2007). A instância sfjs01 representa um problema com flexibilidade total e é constituída por 2 tarefas e cada tarefa composta por 2 operações. Sejam $i=1,2$ as operações da tarefa 1 e $i=3,4$ as operações da tarefa 2 . Além disso, existem duas máquinas para processar as tarefas $(M=\{1,2\})$ e todas as operações podem 
ser executadas por quaisquer das máquinas. Assim, temos que $\mathcal{O}_{1}=\mathcal{O}_{2}=\{1,2,3,4\}$. O tempo de processamento de operação $i$ na máquina $k$ é descrito na Tabela 4.

Tabela 4 - Dados da instância sfjs01 de Fattahi, Mehrabad e Jolai (2007).

\begin{tabular}{clc}
\hline Operação $i$ & \multicolumn{2}{c}{ Tempo de processamento na máquina $k$} \\
\cline { 2 - 3 } & 1 & 2 \\
\hline 1 & 25 & 37 \\
2 & 32 & 24 \\
3 & 45 & 65 \\
4 & 21 & 65 \\
\hline
\end{tabular}

Uma solução factível pode ser obtida atribuindo as operações 1 e 2 à máquina 2 $\left(x_{12}=x_{22}=1\right.$ e $\left.x_{32}=x_{42}=0\right)$ e as operações 3 e 4 à máquina $1\left(x_{11}=x_{21}=0\right.$ e $\left.x_{31}=x_{41}=1\right)$. O diagrama de Gantt da respectiva solução é descrito pela Figura 11 e a Tabela 5 descreve o instante de início e término de processamento de cada operação. O valor do makespan corresponde ao instante de término do processamento da operação 4.

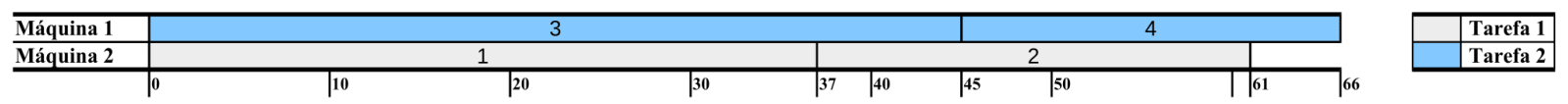

Figura 11 - Diagrama de Gantt de uma solução factível para a instância sfjs01.

Tabela 5 - Valores para as variáveis $s_{i}, p_{i}$ e $c_{i}$ na solução factível da instância sfjs01 de Fattahi, Mehrabad e Jolai (2007).

\begin{tabular}{ccccc}
\hline Operação $i$ & & $s_{i}$ & $p_{i}$ & $c_{i}$ \\
\cline { 1 - 3 } \cline { 5 - 6 } & & 0 & 37 & 37 \\
2 & & 37 & 24 & 61 \\
3 & & 0 & 45 & 45 \\
4 & & 45 & 21 & 66 \\
\hline
\end{tabular}

Considere a inequação válida Half Cuts (inequação (3.32)). Sendo $k=1, S=$ $\{1,2,3,4\}$ e $i=4$, temos que $\underset{j \in S}{\operatorname{Min}} r_{j}=0$, pois, como as operações 1 e 3 representam as primeiras que são executadas em cada tarefa, temos que $r_{1}=r_{3}=0$. Assim, a inequação (3.32) torna-se

$$
\begin{aligned}
s_{4} & \geq y_{141} \cdot p_{11}+y_{241} \cdot p_{21}+y_{341} \cdot p_{31} \\
& =25 \cdot y_{141}+32 \cdot y_{241}+45 \cdot y_{341} .
\end{aligned}
$$

Para a respectiva solução factível, vamos analisar os possíveis valores para as variáveis $y_{141}, y_{241}$ e $y_{341}$ presentes no lado direito da inequação (3.39). Como a operação 3 precede a operação 4 na tarefa 2 e para que a restrição $s_{3}+p_{3}-L \cdot\left(1-y_{341}\right) \leq s_{4}$ (restrição do tipo (3.37)) seja satisfeita, devemos necessariamente ter que $y_{341}=1$. Considerando as operações 1 e 4 , como $x_{41}=1$ e $x_{11}=0$, então a restrição do tipo (3.36) estabelece que $y_{141}+y_{411} \geq 0$. Além disso, a restrição $s_{1}+p_{1}-L \cdot\left(1-y_{141}\right) \leq s_{4}$ não é violada para qualquer escolha da 
variável binária $y_{141}$. Dessa forma, o valor da solução para a variável $y_{141}$ pode ser igual a 1 , mesmo que a operação 1 não seja atribuída à máquina 1 . E de fato isso ocorre ao obter a respectiva solução pelo resolvedor Gurobi. Por fim, devido à restrição do tipo (3.37), o valor da solução para a variável $y_{241}$ deve ser igual a 0 .

Logo, considerando $y_{341}=1, y_{241}=0$ e a possibilidade de $y_{141}=1$, a partir de (3.39), temos que $s_{4} \geq 25+45=70$. Observe que essa inequação não é válida, pois pela solução factível apresentada, temos que $s_{4}=45$. Isso ocorre pois o limitante inferior para o início do processamento da operação 4 contém o tempo de processamento da operação 1, uma vez que o valor da variável $y_{141}$ induz que 1 seja executada antes de 4 na máquina 1 . Assim, faz-se necessário impor que $y_{i j k}$ ou $y_{j i k}$ seja igual a 1 se e somente se ambas as tarefas $i$ e $j$ sejam atribuídas a máquina $k$. Caso $x_{i k}=0$ ou $x_{j k}=0$, então devemos ter que $y_{i j k}=y_{j i k}=0$.

Para satisfazer essas condições, a restrição (3.36) é substituída pela equação

$$
y_{i j k}+y_{j i k}=x_{i k} \cdot x_{j k}, \quad \forall k \in M, \forall(i, j) \in E_{k} .
$$

Como a nova restrição (3.40) contém o termo quadrático $x_{i k} \cdot x_{j k}$, o novo modelo torna-se não-linear. A linearização dessa restrição é obtida substituindo o produto $x_{i k} \cdot x_{j k}$ pela variável binária $z_{i j k}$ e acrescentando ao modelo as restrições

$$
\begin{array}{ll}
z_{i j k} \leq x_{i k}, & \forall k \in M, \forall(i, j) \in E_{k} \\
z_{i j k} \leq x_{j k}, & \forall k \in M, \forall(i, j) \in E_{k} \\
x_{i k}+x_{j k} \leq z_{i j k}+1, & \forall k \in M, \forall(i, j) \in E_{k} \\
z_{i j k} \in\{0,1\}, & \forall k \in M, \forall(i, j) \in E_{k} .
\end{array}
$$

Para verificar que a restrição (3.40) equivale as restrições (3.41)-(3.44), observe que, se $x_{i k}=x_{j k}=1$, então, por (3.41)-(3.42) temos que $z_{i j k} \leq 1 \mathrm{e}$, por (3.43), $z_{i j k} \geq 1$. Logo, $z_{i j k}=1$. Agora, se $x_{i k}=0(1)$ e $x_{j k}=1(0)$ então as inequações $z_{i j k} \leq 0(1), z_{i j k} \leq 1(0) \mathrm{e}$ $z_{i j k} \geq 0$ indicam que $z_{i j k}=0$. Por fim, de forma análoga, se $x_{i k}=x_{j k}=0$, então $z_{i j k}=0$.

Assim, a reformulação de MILP-1 é dada por

$$
\begin{aligned}
& \text { Minimizar } C_{\text {Max }} \\
& \text { Sujeito a } \sum_{k \in \mathscr{M}_{i}} x_{i k}=1, \quad \forall i \in O \\
& p_{i}=\sum_{k \in \mathscr{M}_{i}} x_{i k} \cdot p_{i k}, \quad \forall i \in O \\
& s_{i}+p_{i} \leq C_{\text {Max }}, \quad \forall i \in O \\
& y_{i j k}+y_{j i k}=z_{i j k}, \quad \forall k \in M, \forall(i, j) \in E_{k} \\
& z_{i j k} \leq x_{i k}, \quad \forall k \in M, \forall(i, j) \in E_{k} \\
& z_{i j k} \leq x_{j k}, \quad \forall k \in M, \forall(i, j) \in E_{k} \\
& x_{i k}+x_{j k} \leq z_{i j k}+1, \quad \forall k \in M, \forall(i, j) \in E_{k} \\
& s_{i}+p_{i}-L \cdot\left(1-y_{i j k}\right) \leq s_{j}, \quad \forall k \in M, \forall(i, j) \in E_{k} \\
& s_{i}+p_{i} \leq s_{j}, \quad \forall(i, j) \in P \\
& s_{i} \geq 0, \quad \forall i \in O \\
& x_{i k} \in\{0,1\}, \quad \forall k \in M, \forall i \in \mathcal{O}_{k} \\
& y_{i j k} \in\{0,1\}, \quad \forall k \in M, \forall(i, j) \in E_{k} \\
& z_{i j k} \in\{0,1\}, \quad \forall k \in M, \forall(i, j) \in E_{k}
\end{aligned}
$$

e será denominada de MILP-2. 


\subsubsection{Comparação entre os modelos MILP-1 e MILP-2}

Na sequência apresentamos uma breve comparação dos modelos MILP-1 e MILP-2. As dimensões de MILP-1 e MILP-2 são definidas pelo número de variáveis, denotado por $\mathcal{V}$, e pelo número de restrições, denotado por $\mathcal{C}$, de cada modelo.

As variáveis de decisão de MILP-1 são $C_{M a x}, s_{i}, p_{i}, x_{i k}$ e $y_{i j}$, sendo as três primeiras contínuas e as demais binárias. O número total de variáveis é dado por

$$
\begin{aligned}
\mathcal{V}(M I L P-1) & =1+|O|+|O|+\sum_{i=1}^{|O|}\left|\mathscr{M}_{i}\right|+\sum_{k=1}^{m}\left|\mathcal{O}_{k}\right| \cdot\left(\left|\mathcal{O}_{k}\right|-1\right) \\
& \leq 1+N+N+N m+m N(N-1) \\
& =1+2 N+N^{2} m .
\end{aligned}
$$

O primeiro e o segundo somatórios de $\mathcal{V}(M I L P-1)$ representam o número de variáveis binárias $x_{i k}$ e $y_{i j}$, respectivamente.

O número total de restrições de MILP-1 é dado por

$$
\begin{aligned}
\mathcal{C}(M I L P-1) & =|O|+|O|+|O|+\sum_{k=1}^{m}\left|\mathcal{O}_{k}\right| \cdot\left(\left|\mathcal{O}_{k}\right|-1\right)+\sum_{k=1}^{m}\left|\mathcal{O}_{k}\right| \cdot\left(\left|\mathcal{O}_{k}\right|-1\right)+\sum_{i=1}^{n}\left(n_{i}-1\right) \\
& =3 N+2 \sum_{k=1}^{m}\left|\mathcal{O}_{k}\right| \cdot\left(\left|\mathcal{O}_{k}\right|-1\right)+N-n \\
& \leq 4 N+2 m N(N-1)-n \\
& =4 N+2 N^{2} m-2 N m-n .
\end{aligned}
$$

O valor total de variáveis binárias $x_{i k}$ e $y_{i j}$ e de variáveis contínuas em MILP-2 é o mesmo que em MILP-1. Além disso, em decorrência da reformulação, para cada restrição do tipo (3.40), são adicionadas uma nova variável binária $z_{i j k}$ e três novas restrições (inequações (3.41), (3.42) e (3.43)) ao modelo MILP-2. Dessa forma,

$$
\begin{aligned}
\mathcal{V}(M I L P-2) & =1+|O|+|O|+\sum_{i=1}^{|O|}\left|\mathscr{M}_{i}\right|+\sum_{k=1}^{m}\left|\mathcal{O}_{k}\right| \cdot\left(\left|\mathcal{O}_{k}\right|-1\right)+\sum_{k=1}^{m}\left|\mathcal{O}_{k}\right| \cdot\left(\left|\mathcal{O}_{k}\right|-1\right) \\
& \leq 1+N+N+N m+2 m N(N-1) \\
& =1+2 N+2 N^{2} m-N m \\
\mathcal{C}(M I L P-1) & =|O|+|O|+|O|+5 \sum_{k=1}^{m}\left|\mathcal{O}_{k}\right| \cdot\left(\left|\mathcal{O}_{k}\right|-1\right)+\sum_{i=1}^{n}\left(n_{i}-1\right) \\
& =3 N+5 \sum_{k=1}^{m}\left|\mathcal{O}_{k}\right| \cdot\left(\left|\mathcal{O}_{k}\right|-1\right)+N-n \\
& \leq 4 N+5 m N(N-1)-n \\
& =4 N+5 N^{2} m-5 N m-n .
\end{aligned}
$$




\subsection{Problema de separação}

Uma etapa importante da utilização de inequações válidas em um algoritmo baseado em PC consiste em verificar se tais inequações são violadas pela solução da relaxação do problema ou estabelecer que nenhuma desigualdade violada exista. Essa etapa é denominada de problema de separação.

Na sequência, descrevemos os procedimentos utilizados no nosso algoritmo de separação. A relaxação do problema MILP-2 é obtida desconsiderando as restrições de integralidade das variáveis inteiras $x, y$ e $z$. A solução ótima da relaxação de MILP-2 será denotada por $\left(s^{*}, z^{*}, x^{*}, y^{*}, z^{*}\right)$. Seja $\mathcal{O}_{k} \subseteq \mathcal{O}_{k}$ o conjunto de operações atribuídas à máquina $k$, isto é, $\mathcal{O}_{k}^{*}=\left\{i \in O ; \quad x_{i k}^{*}=1\right\}$.

Nas inequações 3-diciclos e $\mathcal{K}$-fences, para cada máquina $k \in M$, definimos o conjunto de vértices $V$ do digrafo completo $D_{n}=\left(V, A_{n}\right), n=|V|$ como sendo o conjunto $\mathcal{O}_{k}^{*}$.

3-diciclos. As inequações 3-diciclos são geradas enumerando todas as $\left(\begin{array}{l}n \\ 3\end{array}\right)$ possibilidades. Embora a complexidade para esse procedimento seja $O\left(n^{3}\right)$, a seguinte observação aumenta a eficiência da enumeração: se $y_{i j k}^{*}=0$ ou $y_{i j k}^{*}+y_{j z k}^{*} \leq 1$, então sabemos que a inequação não será violada (NEMHAUSER; SAVELSBERGH, 1992).

k-Fences. Se $n \geq 6$ e $n$ for par, o número de total de $k$-fences $\left(3 \leq \kappa \leq \frac{n}{2}\right)$ é igual a

$$
\sum_{k=3}^{\frac{n}{2}}\left(\left(\begin{array}{c}
n \\
2 k
\end{array}\right) \cdot\left(\begin{array}{c}
2 k \\
k
\end{array}\right) \cdot k !\right)=\sum_{k=3}^{\frac{n}{2}} \frac{n !}{(n-2 k) \cdot k !} .
$$

Para cada valor de $\mathcal{K}$, a complexidade para enumerar todos $\mathcal{K}$-fences é $O\left(n^{2 \kappa}\right)$. Como não sabemos se existe algum algoritmo eficiente para resolver o problema da separação para $\kappa$-fences, decidimos abordar somente o caso para $\kappa=3$. O processo de identificar as inequações 3-fences que são violadas consiste de uma heurística proposta por Grötschel, Jünger e Reinelt (1984) em seu algoritmo de PC. Para descrevê-la, considere $F=\left(V_{F}, A_{F}\right)$ um 3-fences em um digrafo completo $D_{6}$ e $c^{T} \in \mathbb{R}^{30}$ seu vetor de incidência. Os autores observaram que, se nenhuma inequação 3-diciclos for violada, então o máximo da função $c^{T} y$ é obtido fixando o valor de $y_{i j}$ em 1 para todo arco piquete $(i, j) \in A_{F}$ e fixando o valor $y_{i j}$ em $\frac{1}{2}$ para todo arco estaca $(i, j) \in A_{F}$. Para os fixados, a respectiva inequação 3 -fences é violada, pois a soma dos componentes de $F$ é 7,5. Assim, para encontrarmos inequações 3 -fences violadas, enumeramos todos os possíveis trios de $\operatorname{arcos}\left(i_{1}, j_{1}\right),\left(i_{2}, j_{2}\right)$ e $\left(i_{3}, j_{3}\right)$ que não possuem extremidades comuns, tal que $0.4 \leq y_{i_{k} j_{k} k}^{*} \leq 0.6, \mathcal{K}=1,2,3$ e verificamos se a inequação correspondente ao 3-fences com esses três arcos como estaca é violada.

Two Jobs. O nosso algoritmo de separação consiste em enumerar todos os pares de operações $i, j \in S$ tal que $r_{i}+p_{i k}>r_{j}$ e $r_{j}+p_{j k}>r_{i}$.

Reverse Two Jobs. Enumeramos todos os pares de operações $i, j \in S$ tal que $q_{i}+p_{i k}>q_{j}$ e $q_{j}+p_{j k}>q_{i}$.

As inequações Half Cuts, Late Job Cuts e as suas "reversas" são definidas para qualquer conjunto $S \subseteq \mathcal{O}_{k}, k \in M$. Definimos inicialmente que $S \subseteq \mathcal{O}_{k}^{*}$, mas mesmo reduzindo a quantidade de operações para $S$, torna-se inviável gerar todos os possíveis subconjuntos contidos em $\mathcal{O}_{k}^{*}$. Dessa forma, o foco do nosso algoritmo de separação é gerar alguns dos possíveis subconjuntos $S$ de $\mathcal{O}_{k}^{*}$.

Half Cuts. Inicialmente as operações em $\mathcal{O}_{k}^{*}$ são ordenadas de forma decrescente de acordo 
com o valor de seus heads. Para cada $i \in \mathcal{O}_{k}^{*}$, nós definimos $S_{i}$ como sendo o conjunto formado pela própria operação $i$ e por todas as operações em $\mathcal{O}_{k}^{*}$ que possuem o valor do head maior que $r_{i}$, e geramos a inequação vinculada a cada operação de $S_{i}$. Primeiramente, o conjunto $S_{i}$ é formado por uma única operação, aquela de maior head em $\mathcal{O}_{k}^{*}$. De forma incremental, ampliamos o conjunto $S$ conforme a ordenação adotada em $\mathscr{O}_{k}^{*}$.

Reverse Half Cuts. Ordenando de forma decrescente pelo valor do tail das operações em $\mathcal{O}_{k}^{*}$, a heurística de separação é análoga à utilizada pelas inequações half cuts.

Late Job Cuts e Reverse Late Job Cuts. Considere $S \subseteq \mathcal{O}_{k}^{*}$ tal que $|S|=1$ (por exemplo, $S$ é constituído pela primeira operação do conjunto $\mathscr{O}_{k}^{*}$ ). A nossa heurística consiste, de forma incremental, em acrescentar operações ao conjunto $S$ até que $S=\mathcal{O}_{k}^{*}$. A cada acréscimo, considerando $i \in S$ e $l \in \mathcal{O}_{k}^{*}$, determinamos todas as possíveis inequações e verificamos quais foram violadas.

\subsection{Experimentos computacionais}

Nesta seção, apresentamos os métodos implementados e os resultados obtidos. Para a resolução dos problemas de otimização, foi utilizado o resolvedor Gurobi, versão 5.6. As abordagens apresentadas nesta seção foram implementadas na linguagem Java e executadas em um computador Intel i7 com processador 1.80 GHz (8 GB RAM).

Dividimos a seção em três partes. Inicialmente, descrevemos os conjuntos de problemas utilizados nos experimentos e apresentamos as dimensões de cada instância nos modelos MILP1 e MILP-2. As instâncias teste foram modeladas através da linguagem MPL (Mathematical Programming System) (MAXIMA, 2014). Posteriormente, com intuito de utilizar as inequações válidas descritas na seção $3.2 \mathrm{em}$ um algoritmo $\mathrm{B} \& \mathrm{C}$, relatamos o desempenho destes cortes em um algoritmo de PC. Na última parte, comparamos os resultados do nosso algoritmo $\mathrm{B} \& \mathrm{C}$ com o resolvedor Gurobi.

Com a intenção de facilitar a leitura, nas tabelas desta tese que apresentam algum dado com, no mínimo, 6 dígitos inteiros, utilizamos o símbolo " ." como separador de milhares em todos os seus valores.

\subsubsection{Conjunto de instâncias teste}

Apresentamos os quatro conjuntos de instâncias teste utilizados nos experimentos computacionais: Fdata, BRdata, BCdata e DPdata. Além de apontar as características dos problemas, comparamos o número de restrições e o número de variáveis binárias e contínuas nos modelos MILP-1 e MILP-2. Uma descrição minuciosa destes problemas pode ser encontrada no trabalho de Behnke e Geiger (2012). Com exceção das instâncias do conjunto Fdata, todas as demais podem ser obtidas em Mastrolilli (2016).

Os modelos MILP-1 e MILP-2 utilizam o parâmetro $L$ para indicar um número suficientemente grande e este deve ser um limitante superior para o makespan ótimo. Para isso, o valor de $L$ é definido como sendo a soma, para cada operação, do maior tempo de processamento dentre todas as máquinas habilitadas a processá-la. 


\subsubsection{Conjunto Fdata}

Fattahi, Mehrabad e Jolai (2007) propõem um conjunto de 20 instâncias para o JSF com o objetivo de minimizar o makespan. Esse conjunto é denominado Fdata e seus problemas são divididos em dois grupos: 10 instâncias de pequeno porte (sfjs1-sfjs10) e 10 de médio e grande porte (mfjs1-mfjs10). Dentre os conjuntos de instâncias apresentados nesta seção, Fdata é o único que não está disponível em Mastrolilli (2016), mas pode ser facilmente obtido através da página pessoal dos autores.

A Tabela 6 descreve os dados do conjunto Fdata e as dimensões dos modelos MILP-1 e MILP-2. Cada instância, em seu conjunto, é definida pelo seu nome e pelo terno $(i, j, k)$, em que $i$ representa o número de tarefas, $j$ o número de operações para cada tarefa e $k$ o número de máquinas. Como era esperado, devido à nova definição da variável binária y no modelo MILP-2, em 90\% dos problemas, o número de variáveis binárias em MILP-2 é pelo menos o dobro do que a quantidade do modelo MILP-1. Além disso, em decorrência da linearização da restrição do tipo (3.40), MILP-2 também apresenta um número superior de restrições. A quantidade de variáveis contínuas é a mesma em ambos os modelos.

\subsubsection{Conjunto BRdata}

O conjunto BRdata de Brandimarte (1993) apresenta 15 instâncias para a minimização do makespan e 5 instâncias para a minimização do atraso total ponderado. Das 15 instâncias relacionadas ao makespan, utilizamos nos testes computacionais apenas 10 (MK01-MK10), uma vez que são as únicas disponíveis em Mastrolilli (2016). Não conseguimos o acesso das 5 instâncias restantes.

$\mathrm{Na}$ Tabela 7, a primeira coluna indica o nome da instância de teste. Além da notação utilizada na Tabela 6 , o símbolo $\left|\mathscr{M}_{i j}\right|$ presente na coluna "Dimensão" denota o número de máquinas habilitadas para processar uma operação. Os valores referentes ao item $j$ indicam o número mínimo e máximo de operações por tarefa. O tempo de processamento das operações em cada máquina foi gerado por distribuição uniforme dentro de um intervalo definido pelos autores. As demais colunas representam a dimensão de cada modelo. Para os problemas MK03, MK06 e MK07, o número de variáveis binárias no modelo MILP-2 é superior ao triplo do número de variáveis binárias em MILP-1. Em relação ao número de restrições, a quantidade encontrada em MILP-2 é pelo menos $240 \%$ do número de restrições de MILP-1. Essa porcentagem também está presente nos dois próximos conjuntos.

\subsubsection{Conjunto BCdata}

O conjunto BCdata de Barnes (1996) é composto por 21 instâncias e dividido em três grupos. As instâncias destes grupos foram construídas através de três problemas clássicos para JS: mt10, la24 e la40. O primeiro grupo (mt10) é baseado em uma instância introduzida no trabalho de Fisher e Thompson (1963). O segundo e terceiro grupos (setb4 e seti5) são baseados, respectivamente, nas instâncias la24 e la40, e introduzidos por Lawrence (1984).

Para adaptar os problemas ao contexto do JSF, as máquinas foram replicadas segundo dois critérios: o tempo total de processamento requerido por uma máquina e a cardinalidade de cada máquina no caminho crítico da melhor solução do JS. O processo de replicar uma máquina consiste em criar uma nova máquina para processar uma operação, mas com o 
Tabela 6 - Descrição do conjunto Fdata e do número de variáveis e restrições dos modelos MILP-1 e MILP-2.

\begin{tabular}{|c|c|c|c|c|c|c|c|}
\hline \multirow[t]{2}{*}{ Problema } & \multirow{2}{*}{$\frac{\text { Dimensão }}{(i, j, k)}$} & \multicolumn{3}{|c|}{ MILP-1 } & \multicolumn{3}{|c|}{ MILP-2 } \\
\hline & & Binárias & Contínuas & Restrições & Binárias & Contínuas & Restrições \\
\hline sfjs01 & $(2,2,2)$ & 20 & 9 & 62 & 56 & 9 & 134 \\
\hline sfjs02 & $(2,2,2)$ & 16 & 9 & 38 & 30 & 9 & 74 \\
\hline sfjs03 & $(3,2,2)$ & 38 & 13 & 101 & 90 & 13 & 221 \\
\hline sfjs04 & $(3,2,2)$ & 40 & 13 & 105 & 94 & 13 & 231 \\
\hline sfjs05 & $(3,2,2)$ & 42 & 13 & 141 & 132 & 13 & 321 \\
\hline sfjs06 & $(3,3,2)$ & 65 & 19 & 157 & 139 & 19 & 343 \\
\hline sfjs07 & $(3,3,5)$ & 62 & 19 & 129 & 114 & 19 & 273 \\
\hline sfjs08 & $(3,3,4)$ & 74 & 19 & 165 & 150 & 19 & 363 \\
\hline sfjs09 & $(3,3,3)$ & 90 & 19 & 225 & 210 & 19 & 513 \\
\hline sfjs10 & $(4,3,5)$ & 76 & 25 & 164 & 140 & 25 & 344 \\
\hline mfjs01 & $(5,3,6)$ & 147 & 31 & 359 & 337 & 31 & 815 \\
\hline mfjs02 & $(5,3,7)$ & 173 & 31 & 439 & 423 & 31 & 1015 \\
\hline mfjs03 & $(6,3,7)$ & 254 & 37 & 674 & 656 & 37 & 1586 \\
\hline mfjs04 & $(7,3,7)$ & 330 & 43 & 897 & 876 & 43 & 2127 \\
\hline mfjs05 & $(7,3,7)$ & 341 & 43 & 877 & 855 & 43 & 2077 \\
\hline mfjs06 & $(8,3,7)$ & 428 & 49 & 1124 & 1098 & 49 & 2678 \\
\hline mfjs07 & $(8,4,7)$ & 704 & 65 & 1840 & 1798 & 65 & 4420 \\
\hline mfjs08 & $(9,4,8)$ & 794 & 73 & 1979 & 1930 & 73 & 4745 \\
\hline mfjs09 & $(11,4,8)$ & 1141 & 89 & 2885 & 2823 & 89 & 6965 \\
\hline mfjs10 & $(12,4,8)$ & 1374 & 97 & 3472 & 3404 & 97 & 8410 \\
\hline
\end{tabular}

mesmo tempo de processamento. As sete instâncias de cada grupo foram geradas conforme os critérios descritos pela Tabela 8.

A Tabela 9 apresenta uma visão geral dos problemas do BCdata. O número de tarefas para os problemas varia entre 10 e 20, o número de máquinas entre 5 e 10 e o número de operações para cada tarefa entre 15 e 25 . Considerando o número total de operações para cada problema, o seu valor varia entre 196 e 387. 
Tabela 7 - Descrição do conjunto BRdata e do número de variáveis e restrições dos modelos MILP-1 e MILP-2.

\begin{tabular}{|c|c|c|c|c|c|c|c|}
\hline \multirow[t]{2}{*}{ Problema } & \multirow{2}{*}{$\begin{array}{l}\text { Dimensão } \\
\left(i, j, k,\left|\mathscr{M}_{i j}\right|\right)\end{array}$} & \multicolumn{3}{|c|}{ MILP-1 } & \multicolumn{3}{|c|}{ MILP-2 } \\
\hline & & Binárias & Contínuas & Restrições & Binárias & Contínuas & Restrições \\
\hline MK01 & $(10,5-7,6,3)$ & 2.051 & 111 & 5.226 & 5.131 & 111 & 12.750 \\
\hline MK02 & $(10,5-7,6,6)$ & 3.288 & 117 & 18.802 & 18.818 & 117 & 46.672 \\
\hline MK03 & $(15,10-10,8,5)$ & 16.517 & 301 & 51.541 & 51.407 & 301 & 127.975 \\
\hline MK04 & $(15,3-10,8,3)$ & 3.844 & 181 & 9.065 & 8.892 & 181 & 22.145 \\
\hline MK05 & $(15,5-10,4,2)$ & 7.321 & 213 & 16.937 & 16.709 & 213 & 41.729 \\
\hline MK06 & $(10,15-15,15,5)$ & 17.840 & 301 & 54.610 & 54.510 & 301 & 135.640 \\
\hline MK07 & $(20,5-5,5,5)$ & 8.499 & 201 & 32.124 & 32.027 & 201 & 79.740 \\
\hline MK08 & $(20,5-15,10,2)$ & 12.236 & 451 & 26.624 & 26.066 & 451 & 65.240 \\
\hline MK09 & $(20,10-15,10,5)$ & 30.028 & 481 & 79.608 & 79.274 & 481 & 197.610 \\
\hline MK10 & $(20,10-5,10,2)$ & 37.792 & 481 & 105.972 & 105.748 & 481 & 263.520 \\
\hline
\end{tabular}

Tabela 8 - Descrição da construção dos problemas do conjunto BCdata.

\begin{tabular}{|c|c|}
\hline Problema & Explicação \\
\hline$-\mathrm{x}$ & $\begin{array}{l}\text { A máquina com maior tempo de processamento acumulado é } \\
\text { replicada uma vez }\end{array}$ \\
\hline$-x x$ & $\begin{array}{l}\text { A máquina com maior tempo de processamento acumulado é } \\
\text { replicada duas vezes }\end{array}$ \\
\hline$-x x x$ & $\begin{array}{l}\text { A máquina com maior tempo de processamento acumulado é } \\
\text { replicada três vezes }\end{array}$ \\
\hline$-x y$ & $\begin{array}{l}\text { As máquinas com os dois maiores tempos de processamento } \\
\text { acumulados são replicadas uma vez }\end{array}$ \\
\hline$-x y z$ & $\begin{array}{l}\text { As máquinas com os três maiores tempos de processamento } \\
\text { acumulados são replicadas uma vez }\end{array}$ \\
\hline$-\mathrm{c}$ & $\begin{array}{l}\text { A máquina com o maior número de operações no caminho crítico } \\
\text { é replicada uma vez }\end{array}$ \\
\hline$-\mathrm{cc}$ & $\begin{array}{l}\text { As máquinas com os dois maiores números de operações no caminho crítico } \\
\text { são replicadas uma vez }\end{array}$ \\
\hline
\end{tabular}

Fonte: adaptado de Behnke e Geiger (2012)

Tabela 9 - Descrição do conjunto BCdata e do número de variáveis e restrições dos modelos MILP-1 e MILP-2.

\begin{tabular}{|c|c|c|c|c|c|c|c|}
\hline \multirow[t]{2}{*}{ Problema } & \multirow{2}{*}{$\frac{\text { Dimensão }}{(i, j, k)}$} & \multicolumn{3}{|c|}{ MILP-1 } & \multicolumn{3}{|c|}{ MILP-2 } \\
\hline & & Binárias & Contínuas & Restrições & Binárias & Contínuas & Restrições \\
\hline mt10c1 & $(10,10,11)$ & 1010 & 201 & 2370 & 2090 & 201 & 5340 \\
\hline mt10cc & $(10,10,12)$ & 1020 & 201 & 2550 & 2280 & 201 & 5790 \\
\hline $\operatorname{mt10x}$ & $(10,10,11)$ & 1010 & 201 & 2370 & 2090 & 201 & 5340 \\
\hline $\operatorname{mt10xx}$ & $(10,10,12)$ & 1020 & 201 & 2550 & 2280 & 201 & 5790 \\
\hline $\operatorname{mt10xxx}$ & $(10,10,13)$ & 1030 & 201 & 2730 & 2470 & 201 & 6240 \\
\hline mt10xy & $(10,10,12)$ & 1020 & 201 & 2550 & 2280 & 201 & 5790 \\
\hline
\end{tabular}




\subsubsection{Conjunto DPdata}

O conjunto DPdata proposto por Dauzère-Pérès e Paulli (1997) é composto por 18 instâncias. A dimensão de cada instância está descrita na Tabela 10. O tempo de processamento é definido dentro de um específico intervalo e o número de máquinas habilitadas a processar uma operação é definido por uma função de probabilidade. Se nenhuma máquina for atribuída a uma operação, uma máquina é escolhida de forma aleatória. Todos os parâmetros foram gerados aleatoriamente a partir de uma distribuição uniforme dentro de um intervalo pré-fixado. A propriedade mais marcante destes problemas é o fato de que o número de operações por tarefa é sempre superior ao número de máquinas. Em relação às instâncias dos demais conjuntos, os do conjunto DPdata apresentam, em sua maioria, a maior quantidade de variáveis e restrições.

Tabela 10 - Descrição do conjunto DPdata e do número de variáveis e restrições dos modelos MILP-1 e MILP-2.

\begin{tabular}{|c|c|c|c|c|c|c|c|}
\hline \multirow[t]{2}{*}{ Problema } & \multirow{2}{*}{$\frac{\text { Dimensão }}{(i, j, k)}$} & \multicolumn{3}{|c|}{ MILP-1 } & \multicolumn{3}{|c|}{ MILP-2 } \\
\hline & & Binárias & Contínuas & Restrições & Binárias & Contínuas & Restrições \\
\hline $01 \mathrm{a}$ & $(10,15-25,5)$ & 9.813 & 393 & 20.122 & 19.569 & 393 & 49.144 \\
\hline $02 \mathrm{a}$ & $(10,15-25,5)$ & 19.358 & 393 & 44.602 & 44.160 & 393 & 110.344 \\
\hline $03 \mathrm{a}$ & $(10,15-25,5)$ & 31.067 & 393 & 100.570 & 100.297 & 393 & 250.264 \\
\hline $04 a$ & $(10,15-25,5)$ & 9.813 & 393 & 20.122 & 19.569 & 393 & 49.144 \\
\hline $05 \mathrm{a}$ & $(10,15-25,5)$ & 19.358 & 393 & 44.602 & 44.160 & 393 & 110.344 \\
\hline $06 \mathrm{a}$ & $(10,15-25,5)$ & 31.067 & 393 & 100.570 & 100.297 & 393 & 250.264 \\
\hline $07 \mathrm{a}$ & $(15,15-25,8)$ & 16.682 & 587 & 34.333 & 33.540 & 587 & 84.097 \\
\hline $08 \mathrm{a}$ & $(15,15-25,8)$ & 47.682 & 587 & 125.709 & 125.260 & 587 & 312.537 \\
\hline $09 a$ & $(15,15-25,8)$ & 78.472 & 587 & 348.713 & 348.738 & 587 & 870.047 \\
\hline $10 \mathrm{a}$ & $(15,15-25,8)$ & 16.682 & 587 & 34.333 & 33.540 & 587 & 84.097 \\
\hline $11 \mathrm{a}$ & $(15,15-25,8)$ & 47.682 & 587 & 125.709 & 125.260 & 587 & 312.537 \\
\hline $12 \mathrm{a}$ & $(15,15-25,8)$ & 78.472 & 587 & 348.713 & 348.738 & 587 & 870.047 \\
\hline $13 \mathrm{a}$ & $(20,20-25,10)$ & 26.778 & 775 & 55.328 & 54.318 & 775 & 136.028 \\
\hline $14 \mathrm{a}$ & $(20,20-25,10)$ & 94.700 & 775 & 268.444 & 268.072 & 775 & 668.818 \\
\hline $15 \mathrm{a}$ & $(20,20-25,10)$ & 143.481 & 775 & 753.384 & 753.797 & 775 & 1.881 .168 \\
\hline $16 \mathrm{a}$ & $(20,20-25,10)$ & 26.778 & 775 & 55.328 & 54.318 & 775 & 136.028 \\
\hline $17 \mathrm{a}$ & $(20,20-25,10)$ & 94.700 & 775 & 268.444 & 268.072 & 775 & 668.818 \\
\hline $18 \mathrm{a}$ & $(20,20-25,10)$ & 143.481 & 775 & 753.384 & 753.797 & 775 & 1.881 .168 \\
\hline
\end{tabular}




\subsubsection{Breve resumo dos conjuntos de instâncias teste}

Com o intuito de comparar as dimensões das instâncias, a Tabela 11 apresenta, para cada conjunto de problemas teste, os valores mínimos, máximos e da média para o número total de variáveis binárias, contínuas e restrições no modelo MILP-1. Utilizamos o modelo MILP-1 apenas como referência para destacar quais conjuntos são de maior ou menor porte.

Tabela 11 - Valores mínimos, máximos e médios do número de variáveis binárias, contínuas e restrições para o modelo MILP-1.

\begin{tabular}{|c|c|c|c|c|c|c|c|c|c|c|c|}
\hline \multirow[t]{2}{*}{ Conjunto } & \multicolumn{3}{|c|}{ Binária } & \multicolumn{3}{|c|}{ Contínuas } & \multicolumn{3}{|c|}{ Restrições } & \multicolumn{2}{|c|}{ Instância } \\
\hline & Mín. & Máx. & Méd. & Mín. & Máx. & Méd. & Mín. & Máx. & Méd. & Mín. & Máx. \\
\hline Fdata & 16 & 1.374 & 310,25 & 9 & 97 & 35,80 & 38 & 3.472 & 791,65 & sfjs02 & mfjs10 \\
\hline BRdata & 2.051 & 37.792 & $13.941,60$ & 111 & 481 & 283,80 & 5.226 & 105.972 & $40.050,90$ & MK01 & Mk10 \\
\hline BCdata & 1.010 & 3.420 & 2.235 & 201 & 451 & 317,67 & 2.370 & 8.445 & 5.400 & $\mathrm{mt10c1}$ & seti5xxx \\
\hline DPdata & 9.813 & 143.481 & $52.003,67$ & 393 & 775 & 585 & 20.122 & 753.384 & $194.578,33$ & $01 a$ & $18 \mathrm{a}$ \\
\hline
\end{tabular}

Conforme os dados da Tabela 11, a instância sfjs02 do conjunto Fdata é a que apresenta a menor quantidade de variáveis binárias, contínuas e restrições para o modelo MILP-1, dentre todas as instâncias teste. Por ser considerado de pequeno porte, o conjunto Fdata é preferido para análise de resultados em modelos MILP.

Considerando o valor máximo para dimensões de MILP-1 em cada conjunto, o menor valor ocorre para a instância mfjs10, também do conjunto Fdata, e o maior valor para a instância 18a do conjunto DPdata. O conjunto Fdata é considerado do menor porte, seguido pelo conjunto BCdata. Como veremos na sequência deste capítulo, considerando todas as instâncias destes dois conjuntos, em 70,73\% dos casos a solução ótima é obtida pelo Gurobi em menos de uma hora de processamento.

Os conjuntos de maior porte e, consequentemente, os que apresentam a maior dimensão para MILP-1 são BRdata e DPdata. Em BRdata e DPdata ainda existem problemas em aberto, isto é, instâncias em que a solução ótima não foi encontrada ou não foi possível provar a sua otimalidade. Os problemas em abertos são: MK10, 05a, 06a, 07a, 10a, 11a, 12a, 13a, 16a, 17a e 18a (RESULTS-QUINTIQ, 2016). Voltaremos a abordar tais problemas na seção 3.5.3.1.

Avaliamos a qualidade do limitante inferior obtido pelo valor ótimo da relaxação dos modelos MILP-1 e MILP-2. Em todos os conjuntos, o valor do limitante para cada problema é o mesmo em ambos os modelos. Dessa forma, o acréscimo de novas restrições ao modelo MILP-2 não trouxe melhorias para o valor do limitante obtido pela relaxação do modelo.

\subsubsection{Resultados do algoritmo de planos de corte}

Nesta seção, apresentamos os resultados obtidos pelo algoritmo de PC implementado nesta tese. O objetivo da implementação é analisar o comportamento e a qualidade do limitante inferior obtido pelas classes de inequações válidas, e identificar se existe alguma família com melhor desempenho comparada com as demais. 
O algoritmo de PC é brevemente descrito na sequência. Inicialmente, nós consideramos a relaxação de MILP-2. Em cada iteração, o PL corrente é resolvido e acrescentamos ao seu sistema linear as inequações que são violadas pela solução ótima. O valor da solução ótima em cada iteração é um limitante inferior para a solução ótima do JSF.

Em nosso procedimento, o algoritmo é finalizado quando não for possível encontrar uma desigualdade violada ou quando o tempo de processamento for superior a 3600 segundos. A solução ótima da relaxação do modelo em cada iteração do algoritmo de PC foi obtida pelas bibliotecas do software de otimização Gurobi, versão 5.6, com a sua configuração padrão. Nesta configuração, o PL corrente é resolvido através de um processo denominado otimização concorrente. Esse procedimento, conforme o número de processadores do computador, utiliza vários algoritmos ao mesmo tempo, e cada um deles requer uma cópia do PL. Por exemplo, para o caso de quatro threads, a primeira delas utiliza o algoritmo Dual Simplex e as demais o Método de Barreira (GUROBI, 2016).

A primeira análise foi em relação à eficiência das classes de famílias válidas. Para isso, utilizamos como critério o valor do limitante inferior obtido no final do algoritmo de PC.

As classes de inequações definidas na seção 3.2 foram divididas em 4 grupos. O primeiro grupo (G1) é formado pelas classes 3-Diciclos e 3-Fences e os demais grupos são formados pelas classes Two Jobs e Reverse Two Jobs (grupo G2), Half Cuts e Reverse Half Cuts (grupo G3) e Late Jobs e Reverse Late Jobs (grupo G4). Para cada grupo, foi aplicado o algoritmo de PC, inserindo em cada iteração todas as inequações que foram violadas pela solução ótima corrente. Os resultados obtidos estão apresentados nas Tabelas 12, 13, 14 e 15. Cada uma dessas Tabelas apresenta em suas colunas o nome da instância analisada, o valor da relaxação de MILP-2 (LB MILP-2) e o valor do limitante inferior obtido pelo algoritmo de PC em cada grupo. O melhor valor obtido por PC está destacado em negrito, desde que este seja melhor que a solução ótima da relaxação de MILP-2.

De forma resumida, as Tabelas 16 e 17 mostram a quantidade de inequações geradas em cada classe e cada conjunto de problemas, a porcentagem de inequações violadas e o número médio de iterações do algoritmo de PC.

Dos 20 problemas do conjunto Fdata, o algoritmo de PC melhorou o limitante de 7 problemas (Tabela 12). A heurística de separação não encontrou inequação válida para a classe 3-Fences. Embora o algoritmo tenha encontrado muitas inequações da classe 3-Diciclos, a proporção de inequações violadas é bem baixa (Tabela 16). Estas duas características implicaram em um número baixo de iterações do algoritmo e uma má qualidade do limitante. As inequações válidas do Grupo 2 melhoram o limitante inferior somente nos problemas sfj03 e mfjs05 e a heurística de separação não encontrou inequações violadas em apenas 1 problema, o sfs01 para a classe Reverse Two Jobs. As inequações dos grupos 3 e 4 apresentaram a maior quantidade de violações (Tabela 17) e os melhores resultados para o limitante.

Para o conjunto BRdata (Tabela 13), embora a maior quantidade de inequações encontradas seja da classe 3-Diciclos, poucas estavam violadas. A heurística de separação encontrou inequações válidas da classe 3-Fences para os problemas MK03, MK06, MK08 e MK09, mas nenhuma violada. As inequações do grupo 2 melhoram o valor limitante nos problemas MK03, MK06, MK07 e MK09, porém com desempenho inferior as dos grupos 3 e 4. De forma geral, o algoritmo melhorou o limitante em todos os problemas e obteve a solução ótima inteira para os problemas MK03 (para os grupos 3 e 4) e MK08 (para o grupo $3)$.

As inequações dos grupos 1 e 2 não melhoraram o valor do limitante inferior para 
Tabela 12 - Resultado do algoritmo de planos de corte para o conjunto Fdata.

\begin{tabular}{|c|c|c|c|c|c|}
\hline Problema & LB-MILP-2 & Grupo 1 & Grupo 2 & Grupo 3 & Grupo 4 \\
\hline & & LB & LB & LB & LB \\
\hline sfjs01 & 66 & 66 & 66 & 66 & 66 \\
\hline sfjs02 & 107 & 107 & 107 & 107 & 107 \\
\hline sfjs03 & 168 & 168 & 171,38 & 174,63 & 174,63 \\
\hline sfjs04 & 272 & 272 & 272 & 272 & 275,87 \\
\hline sfjs05 & 100 & 100 & 100 & 100 & 100 \\
\hline sfjs06 & 310 & 310 & 310 & 310 & 310 \\
\hline sfjs07 & 397 & 397 & 397 & 397 & 397 \\
\hline sfjs08 & 216 & 216 & 216 & 216 & 216 \\
\hline sfjs09 & 210 & 210 & 210 & 210 & 210 \\
\hline sfjs10 & 427 & 427 & 427 & 427,94 & 427 \\
\hline mfjs01 & 403 & 403 & 403 & 403 & 403 \\
\hline mfjs02 & 396 & 396 & 396 & 396 & 396 \\
\hline mfjs03 & 396 & 396 & 396 & 396,71 & 396,71 \\
\hline mfjs04 & 496 & 496 & 496 & 496 & 496 \\
\hline mfjs05 & 414 & 414 & 418,47 & 422,72 & 421,29 \\
\hline mfjs06 & 614 & 614 & 614 & 614 & 614 \\
\hline mfjs07 & 764 & 764 & 764 & 764 & 764 \\
\hline mfjs08 & 764 & 764 & 764 & 764 & 764 \\
\hline mfjs09 & 764 & 764 & 770 & 783,10 & 778,91 \\
\hline mfjs10 & 944 & 944 & 944 & 944 & 944,10 \\
\hline
\end{tabular}

os problemas do conjunto BCdata (Tabela 14). Entretanto, as do grupo 3 aumentaram o valor do limitante em todas as instâncias e as do grupo 4, em 14 problemas. Exceto para os problemas setb4xy e setb4xyz, o maior valor do limitante foi obtido somente pelas inequações do grupo 3. Em relação ao número de inequações violadas, durante o processamento das 21 instâncias foram geradas 4.680.155 inequações 3-Diciclos, mas apenas $1 \%$ foi violada. Além disso, a nossa heurística não identificou nenhuma inequação da classe 3-Fences. No grupo 2, do total de inequações válidas geradas, $20 \%$ das inequações Two Jobs e $51 \%$ das inequações Two Jobs Reverse foram violadas. No entanto, o uso de tais inequações violadas no algoritmo de PC não proporcionou melhoria no limitante inferior.

Por fim, conforme a Tabela 15, de um total de 18 instâncias do conjunto DPdata, o algoritmo de PC melhorou o limitante de 11 delas. Nenhuma inequação encontrada da classe 3-Diciclo estava violada e, assim como em Fdata e BCdata, nenhuma inequação válida da classe 3-Fence foi identificada (Tabela 16). Embora o algoritmo tenha encontrado inequações 
Tabela 13 - Resultado do algoritmo de planos de corte para o conjunto BRdata.

\begin{tabular}{|c|c|c|c|c|c|}
\hline Problema & LB-MILP-2 & Grupo 1 & Grupo 2 & Grupo 3 & Grupo 4 \\
\hline & & LB & LB & LB & LB \\
\hline MK01 & 22 & 22 & 22 & 39 & 39 \\
\hline MK02 & 18 & 18 & 18 & 25 & 25 \\
\hline MK03 & 63 & 63 & 66,19 & 204 & 204 \\
\hline MK04 & 35 & 35 & 35 & 56 & 50,09 \\
\hline MK05 & 59 & 59 & 59 & 127 & 127 \\
\hline MK06 & 33 & 33 & 33,50 & 41,45 & 39,68 \\
\hline MK07 & 44 & 44 & 47,75 & 133 & 133 \\
\hline MK08 & 162 & 162 & 162 & 523 & 162 \\
\hline MK09 & 130 & 130 & 133,75 & 307 & 130 \\
\hline MK10 & 113 & 113 & 113 & 181 & 113 \\
\hline
\end{tabular}

do grupo 2 violadas, sua inclusão na formulação não trouxe aumento do valor do limitante.

De forma geral, o uso das inequações do grupo 1 no algoritmo de PC não melhorou o limitante inferior para nenhuma das 69 instâncias teste. Para as do grupo 2, houve aumento do limitante em 6 instâncias, distribuídas entre os conjuntos Fdata e BCdata. A utilização das inequações do grupo 3 superou o limitante inferior de MILP-2 em 46 instâncias, e para as do grupo 4, em 32 casos. O melhor valor do limitante superior, quando este superou o valor de LB-MILP-2, foi obtido em 35 instâncias pelas inequações do grupo 3, em 3 instâncias pelas inequações do grupo 4 e em 11 instâncias por ambos os grupos.

Diante dos resultados obtidos, podemos destacar que os melhores valores do limitante inferior foram obtidos pelas inequações dos grupos 3 e 4 . Em todos os problemas, o algoritmo foi finalizado quando não foi possível encontrar uma inequações violada. Embora as inequações 3-Diciclos foram as mais identificadas no algoritmo de PC, o seu uso não trouxe melhoria para o valor do limitante inferior. O algoritmo identificou poucas inequações 3-Fences e, dentre as encontradas, nenhuma foi violada. Um primeiro questionamento a respeito da baixa quantidade de inequações da classe 3-Fences poderia ser em relação à heurística utilizada na etapa de separação. Para isso, enumeramos todas as possíveis inequações 3-Fences para os problemas do conjunto Fdata e o número de inequações ainda permaneceu baixo, sem violação pela solução corrente. Encontramos o uso das inequações 3-Fences na tese de doutorado de Zabala (2006). A autora utiliza o algoritmo B\&C para resolver o Problema de Roteamento de Veículos e o número de inequações violadas pela classe 3-Fences também é bem baixo.

Como nosso objetivo inicial consiste em analisar a eficiência das classes das inequações válidas, identificamos as inequações das classes dos grupos 3 e 4 com os melhores desempenhos, umas vez que estas proporcionaram o maior aumento do limitante inferior. Portanto, segundo este critério, utilizamos somente as classes destes grupos no algoritmo B\&C. 
Tabela 14 - Resultado do algoritmo de planos de corte para o conjunto BCdata.

\begin{tabular}{|c|c|c|c|c|c|}
\hline Problema & LB-MILP-2 & Grupo 1 & Grupo 2 & Grupo 3 & Grupo 4 \\
\hline & & LB & LB & LB & LB \\
\hline mt10c1 & 655 & 655 & 655 & 822,97 & 805,37 \\
\hline mt10cc & 655 & 655 & 655 & 808 & 796 \\
\hline $\operatorname{mt} 10 \mathrm{x}$ & 655 & 655 & 655 & 822,62 & 806,20 \\
\hline $\operatorname{mt10xx}$ & 655 & 655 & 655 & 822,62 & 806,20 \\
\hline $\operatorname{mt10xxx}$ & 655 & 655 & 655 & 822,62 & 806,20 \\
\hline mt10xy & 655 & 655 & 655 & 822,60 & 800,69 \\
\hline mt10xyz & 655 & 655 & 655 & 791,13 & 750,30 \\
\hline setb4c9 & 704 & 704 & 704 & 881 & 872 \\
\hline setb4cc & 704 & 704 & 704 & 881 & 872 \\
\hline setb4x & 704 & 704 & 704 & 881 & 872 \\
\hline setb4xx & 704 & 704 & 704 & 881 & 872 \\
\hline setb4xxx & 704 & 704 & 704 & 881 & 872 \\
\hline setb4xy & 704 & 704 & 704 & 872 & 872 \\
\hline setb4xyz & 704 & 704 & 704 & 872 & 872 \\
\hline seti5c12 & 955 & 955 & 955 & 1118 & 955 \\
\hline seti5cc & 955 & 955 & 955 & 1014 & 955 \\
\hline seti5x & 955 & 955 & 955 & 1170 & 955 \\
\hline seti5xx & 955 & 955 & 955 & 1170 & 955 \\
\hline seti5xxx & 955 & 955 & 955 & 1170 & 955 \\
\hline seti5xy & 955 & 955 & 955 & 1014 & 955 \\
\hline seti5xyz & 955 & 955 & 955 & 1014 & 955 \\
\hline
\end{tabular}

\subsubsection{Resultados do algoritmo branch and cut}

A partir dos resultados obtidos na seção anterior, esta seção tem como objetivo principal analisar a eficiência das classes de inequações válidas dos grupos 3 e 4 em um algoritmo B\&C. Para isso, comparamos os resultados obtidos pelo algoritmo B\&C com os obtidos pelo modelo MILP-1, usando o Gurobi em sua configuração padrão (MILP-1-Gurobi). Embora o número de variáveis binárias e o número de restrições sejam maiores em MILP-2, em relação a MILP-1, também comparamos as soluções obtidas pelos dois modelos usando o Gurobi. Os resultados são apresentados na sequência.

$\mathrm{O}$ algoritmo B\&C é um método exato que combina as abordagens B\&B e PC. Em cada nó da árvore de decisão, é executado o método de PC sobre a relaxação linear do subproblema corrente. Dessa forma, espera-se melhorar o valor do limitante inferior, permitindo podar 
Tabela 15 - Resultado do algoritmo de planos de corte para o conjunto DPdata.

\begin{tabular}{|c|c|c|c|c|c|}
\hline Problema & LB-MILP-2 & Grupo 1 & Grupo 2 & Grupo 3 & Grupo 4 \\
\hline & & LB & LB & $\mathrm{LB}$ & $\mathrm{LB}$ \\
\hline $01 \mathrm{a}$ & 1392 & 1392 & 1392 & 2505 & 2505 \\
\hline $02 \mathrm{a}$ & 1392 & 1392 & 1392 & 1628 & 1462 \\
\hline $03 \mathrm{a}$ & 1392 & 1392 & 1392 & 1392 & 1392 \\
\hline $04 a$ & 1373 & 1373 & 1373 & 2503 & 2503 \\
\hline $05 a$ & 1353 & 1353 & 1353 & 1583 & 1433 \\
\hline $06 \mathrm{a}$ & 1339 & 1339 & 1339 & 1339 & 1339,08 \\
\hline $07 a$ & 1400 & 1400 & 1400 & 2206 & 1400 \\
\hline $08 \mathrm{a}$ & 1400 & 1400 & 1400 & 1400 & 1400 \\
\hline $09 a$ & 1400 & 1400 & 1400 & 1400 & 1400 \\
\hline $10 \mathrm{a}$ & 1380 & 1380 & 1380 & 2197 & 1380 \\
\hline $11 \mathrm{a}$ & 1337 & 1337 & 1337 & 1337,34 & 1337 \\
\hline $12 \mathrm{a}$ & 1310 & 1310 & 1310 & 1310 & 1310 \\
\hline $13 \mathrm{a}$ & 1354 & 1354 & 1354 & 2100 & 1354 \\
\hline $14 \mathrm{a}$ & 1354 & 1354 & 1354 & 1354 & 1354 \\
\hline $15 \mathrm{a}$ & 1354 & 1354 & 1354 & 1354 & 1354 \\
\hline $16 \mathrm{a}$ & 1338 & 1338 & 1338 & 2075 & 1338 \\
\hline $17 \mathrm{a}$ & 1291 & 1291 & 1291 & 1291,43 & 1291 \\
\hline $18 \mathrm{a}$ & 1289 & 1289 & 1289 & 1289 & 1289 \\
\hline
\end{tabular}

Tabela 16 - Resultado do algoritmo de planos de corte para os grupos 1 e 2 .

\begin{tabular}{|c|c|c|c|c|c|c|c|c|c|c|}
\hline \multirow[t]{2}{*}{ Conjunto } & \multicolumn{5}{|c|}{ Grupo 1} & \multicolumn{5}{|c|}{ Grupo 2} \\
\hline & 3-Diciclos & Viol. & 3-Fences & Viol. & Iter. & Two Jobs & Viol. & $\begin{array}{l}\text { Two Jobs } \\
\text { Reverse }\end{array}$ & Viol. & Iter. \\
\hline Fdata & 17.429 & $2,44 \%$ & 0 & 0 & 2,7 & 938 & $71,75 \%$ & 951 & $14,20 \%$ & 5,25 \\
\hline BRdata & 2.335 .839 & $2,23 \%$ & 15.488 & 0 & 6 & 7.825 & $34,11 \%$ & 7.745 & $45,45 \%$ & 6,90 \\
\hline BCdata & 4.680 .155 & $1,08 \%$ & 0 & 0 & 3,2 & 11.507 & $22,75 \%$ & 11.013 & $57,19 \%$ & 4,66 \\
\hline DPdata & 728.230 & 0 & 0 & 0 & 1 & 17.284 & $19,74 \%$ & 15.778 & $51,08 \%$ & 6,57 \\
\hline
\end{tabular}

antecipadamente algumas ramificações da árvore de decisão. O método B\&C é descrito em detalhes no Capítulo 1.

Em vez de considerar uma implementação própria do algoritmo B\&C, decidimos utilizar a biblioteca de funções do Gurobi. Denominamos este procedimento de MILP-2-B\&C. 
Tabela 17 - Resultado do algoritmo de planos de corte para os grupos 3 e 4 .

\begin{tabular}{|c|c|c|c|c|c|c|c|c|c|c|}
\hline \multirow[t]{2}{*}{ Conjunto } & \multicolumn{5}{|c|}{ Grupo 3} & \multicolumn{5}{|c|}{ Grupo 4} \\
\hline & Half Cuts & Viol. & $\begin{array}{l}\text { Half Cuts } \\
\text { Reverse }\end{array}$ & Viol. & Iter. & Late Jobs & Viol. & $\begin{array}{l}\text { Late Jbos } \\
\text { Reverse }\end{array}$ & Viol. & Iter. \\
\hline Fdata & 1.663 & $42,75 \%$ & 1.770 & $20,85 \%$ & 3,8 & 6.684 & $60,76 \%$ & 6.684 & $21,11 \%$ & 3,75 \\
\hline BRdata & 17.583 & $36,28 \%$ & 18.214 & $30,23 \%$ & 3,4 & 426.246 & $32,38 \%$ & 426.246 & $26,96 \%$ & 4,5 \\
\hline BCdata & 137.284 & $14,01 \%$ & 137.914 & $9,33 \%$ & 4 & 4.321 .152 & $5,10 \%$ & 4.321 .152 & $7,31 \%$ & 4,67 \\
\hline DPdata & 114.593 & $10,34 \%$ & 115.988 & $6,67 \%$ & 5,76 & 1.673 .085 & $7,38 \%$ & 1.673 .085 & $7,69 \%$ & 7,52 \\
\hline
\end{tabular}

Além da linguagem Java, a interação com o ambiente Gurobi pode ser feita com as linguagens C, C++, .NET, Python, Matlab e R. Em todos os procedimentos desta seção, o Gurobi interrompe o processo de otimização de uma instância se a solução ótima não for obtida em um tempo inferior a 3600 segundos.

O Gurobi utiliza o algoritmo B\&C para resolver MILPs e IPs. O resolvedor apresenta um conjunto de 13 classes gerais de inequações válidas. Essas classes são denominadas de CliqueCuts, CoverCuts, FlowCoverCuts, FlowPathCuts, GomoryPasses, GUBCoverCuts, ImpliedCuts, MIPSepCuts, MIRCuts, ModKCuts, NetworkCuts, SubMIPCuts e ZeroHalfCuts. Também é permitido ativar ou desativar cada uma dessas classes. Se todos os cortes forem desabilitados, o algoritmo se reduz ao método B\&B. Nos procedimentos MILP-1-Gurobi e MILP-2-Gurobi, todas as 13 classes de inequações válidas foram habilitadas.

No Gurobi, novos cortes podem ser incorporados ao modelo através da função callback() e do método addCut(). A chamada callback() é uma função definida pelo usuário a fim de consultar ou modificar o estado de otimização. Por exemplo, passando os argumentos modelo, where e what para a função callback(), é possível consultar (what) o otimizador para obter detalhes sobre o estado da otimização, além de controlar e modificar o comportamento do Gurobi. O argumento where indica o momento em que ocorrerá a chamada durante o processo de otimização, podendo ser, por exemplo, durante a resolução do método simplex, ou na resolução de um nó, ou na atualização da solução incumbente, entre outros.

Conforme comentado no Capítulo 1, além das estratégias inerentes do algoritmo $B \& B$, outras decisões provenientes do método de PC devem ser levadas em consideração durante a implementação do método B\&C.

A primeira estratégia está relacionada com a seleção do nó na árvore de decisão. A escolha do próximo nó a ser examinado é controlada pelo parâmetro BranchDir. Especificamente no caso de um BP, quando a otimização em um nó na árvore de busca está concluída, através da ramificação, dois novos nós são criados. O primeiro filho corresponde ao arredondamento da variável binária para zero (down branch) e o segundo ao arredondamento da variável binária para um (up branch). Dessa forma, o parâmetro BranchDir permite selecionar ou o nó relacionado à ramificação down branch ou o relacionado à ramificação up branch. Se nenhuma preferência for selecionada, o resolvedor escolhe de forma automática o critério.

A etapa da ramificação do B\&C é controlada pelo Gurobi. Embora não seja possível definir qual a variável utilizada na etapa de ramificação, através do parâmetro VarBranch, o 
resolvedor permite estabelecer 4 estratégias: Pseudo Reduced Cost, Pseudo Shadow Price, Maximum Infeasibility e Strong Branching. Em sua configuração padrão, uma estratégia é selecionada de forma automática, dependendo da característica do problema. Sugerimos o trabalho de Achterberg, Koch e Martin (2005) para informações adicionais a respeito de cada uma dessas estratégias.

O algoritmo B\&C possui parâmetros que podem interferir no desempenho do algoritmo. Estes parâmetros definem quantos cortes violados devem ser adicionados à formulação corrente, o momento em que devemos inserir os planos de corte na árvore de busca e a quantidade de iterações do algoritmo de PC em cada nó. Em relação ao número de cortes, o manual de referência do Gurobi recomenda que as inequações violadas devem ser adicionadas com moderação, uma vez que aumentam o tamanho do modelo de programação linear do atual nó e de todos os seus descendentes.

Conforme os resultados obtidos pelo algoritmo de PC da seção 3.5.2, utilizamos em MILP-2-B\&C somente as classes de inequações válidas do Grupo 3 e Grupo 4. A definição do número máximo de cortes $(k)$ adicionados na relaxação do nó e o momento da inserção $(f)$ dos cortes na árvore de busca foi estabelecida a partir do seguinte procedimento. De todas as instâncias em que a solução ótima do MILP-1-Gurobi não foi obtida pelo Gurobi dentro do limite de tempo de 3600 segundos, selecionamos de forma aleatória as instâncias mfjs09, MK02, MK05, MK09, Barnes10, Barnes13, Barnes18, 03a, 07a e 17a (os resultados obtidos pelo Gurobi para o modelo MILP-1 serão descritos na sequência do texto). Não selecionamos para a amostra problemas com tempo de execução inferior a 3600 segundos, pois esperamos que, se MILP-1-Gurobi resolve tais problemas abaixo do limite de tempo, o mesmo ocorra para o algoritmo B\&C e, dessa forma, o valor do gap destes problemas não teria influência na decisão dos parâmetros.

Definimos três valores para cada parâmetro: $k \in\{150,300,500\}$ e $f \in\{500,1500,2500\}$. Por exemplo, se $k=300$, então são inseridos, no máximo, as 150 inequações mais violadas de cada grupo. O valor do parâmetro $f=1500$ indica que, após o acréscimo de inequações violadas no nó raiz, novas inserções serão realizadas a cada 1500 nós explorados na árvore de busca. A Tabela 18 apresenta os valores das médias dos gaps obtidos pelas combinações dos parâmetros para a amostra de instâncias. O valor do gap é determinado pela diferença relativa entre os limitantes superior $\left(C_{\text {Max }}\right)$ e inferior $(L B)$, isto é, $100 \cdot\left(C_{\text {Max }}-L B\right) / C_{\text {Max }}$.

Definimos que a melhor escolha é aquela que possui o menor valor para a média dos gaps. Dessa forma, em MILP-2-B\&C, fixamos os valores 300 e 500 para os parâmetros $k$ e $f$, respectivamente.

Durante a resolução de um MILP, o Gurobi não permite reotimizar o subproblema associado a um nó. Dessa forma, para cada nó selecionado, as inequações violadas podem ser adicionadas ao problema de programação linear corrente uma única vez. Essa etapa equivale a aplicar, no respectivo nó, o método de PC em uma única iteração.

Os principais resultados desta seção estão descritos nas Tabelas 19, 20, 21, 22 e 23 e representam os dados obtidos pelos procedimentos MILP-1-Gurobi, MILP-2-Gurobi e MILP-2-B\&C. Para cada abordagem, a coluna $L B$ indica o valor de limitante inferior e $C_{M a x}$ representa o valor do makespan. Na sequência, estão descritos os valores do gap e do tempo de processamento, em segundos. Em cada Tabela e em cada instância analisada, destacamos as colunas $L B, C_{M a x}$ e gap do método que apresentou o menor valor para o gap. Se nenhuma coluna for destacada, então as três abordagens obtiveram o mesmo valor para o gap.

Do total de 69 instâncias, MILP1-Gurobi encontrou a solução ótima em 30 problemas. 
Tabela 18 - Valor da média dos gaps para cada combinação dos parâmetros $k$ e $f$ no conjunto para as 10 instâncias de teste.

\begin{tabular}{cccc}
\hline \multicolumn{2}{c}{ Parâmetros } & & Média dos gaps (\%) \\
\cline { 1 - 2 }$k$ & $f$ & & \\
\hline 150 & 500 & & 22,06 \\
150 & 1500 & & 21,47 \\
150 & 2500 & & 22,40 \\
$\mathbf{3 0 0}$ & $\mathbf{5 0 0}$ & & $\mathbf{2 0 , 9 4}$ \\
300 & 1500 & 21,25 \\
300 & 2500 & 21,44 \\
500 & 500 & 21,72 \\
500 & 1500 & 21,29 \\
500 & 2500 & 22,58 \\
\hline
\end{tabular}

As instâncias em que a solução ótima foi obtida em MILP-2 são as mesmas obtidas por MILP-1. Comparando somente as abordagens MILP-1-Gurobi e MILP-2-Gurobi, para as 39 instâncias restantes, o segundo procedimento, apesar de possuir um número superior de variáveis binárias e restrições, obteve o melhor gap em 9 problemas e encontrou uma solução factível para 09a e 18a. Uma possível explicação para o fato pode estar relacionado ao acréscimo das restrições (3.41), (3.42), (3.43) e (3.44) ao modelo MILP-2. Conforme comentado na seção 3.3, o modelo MILP-1 pode apresentar várias soluções ótimas. As restrições acrescentadas podem ter quebrado a simetria das soluções e facilitado o processo de otimização.

O procedimento MILP-1 obteve, de forma isolada, o menor valor do gap em 7 instâncias (mfjs10, 06a, 8a, 11a, 12a, 14a e 17a), enquanto MILP-2, para 5 instâncias (mfjs09, seti5cc, seti5xy, 3a e 9a). O valor do makespan para estas 5 instâncias foi melhor que o valor do makespan em MILP-2-B\&C . Isto indica que os planos de corte utilizados pelo Gurobi foram importantes para o cálculo dos limitantes dos problemas no modelo MILP-2.

Em relação ao algoritmo B\&C, além das instâncias em que a solução ótima foi obtida por MILP-1, o nosso procedimento obteve a solução ótima para mais 6 instâncias (MK03, MK04, MK08, setb4x, setb4xxx, seti5c12). Além disso, ao comparar com MILP1-Gurobi, quando a solução ótima não foi encontrada, o algoritmo MILP-2-B\&C melhorou o gap em 19 instâncias e encontrou um limitante superior para as instâncias 9a e 18a.

Nenhuma das abordagens encontrou uma solução factível para a instância 15a. A abordagem MILP1-Gurobi não encontrou um limitante superior para as instâncias 09a e 18a e MILP2-Gurobi também não encontrou um limitante superior para 14a.

De forma resumida, a Tabela 23 apresenta, para cada uma das abordagens e para cada conjunto de instâncias teste, a média dos valores da quantidade de nós visitados (coluna Nós), dos valores dos gaps (coluna Gap) e dos tempos de processamento (coluna Tempo). No 
Tabela 19 - Resultados computacionais de MILP-1-Gurobi, MILP-2-Gurobi e MILP-2-B\&C para o conjunto Fdata.

\begin{tabular}{|c|c|c|c|c|c|c|c|c|c|c|c|c|}
\hline \multirow[t]{2}{*}{ Problema } & \multicolumn{4}{|c|}{ MILP-1-Gurobi } & \multicolumn{4}{|c|}{ MILP-2-Gurobi } & \multicolumn{4}{|c|}{ MILP-2-B\&C } \\
\hline & $\mathrm{LB}$ & $C_{\text {Max }}$ & $\begin{array}{l}\text { Gap } \\
(\%)\end{array}$ & $\begin{array}{l}\text { Tempo } \\
\text { (s) }\end{array}$ & $\mathrm{LB}$ & $C_{\operatorname{Max}}$ & $\begin{array}{l}\text { Gap } \\
(\%)\end{array}$ & $\begin{array}{l}\text { Tempo } \\
\text { (s) }\end{array}$ & LB & $C_{\operatorname{Max}}$ & $\begin{array}{l}\text { Gap } \\
(\%)\end{array}$ & $\begin{array}{l}\text { Tempo } \\
\text { (s) }\end{array}$ \\
\hline $\mathrm{s} 01$ & 66 & 66 & 0 & 0,01 & 66 & 66 & 0 & 0 & 66 & 66 & 0 & 0 \\
\hline sfjs02 & 107 & 107 & 0 & 0,01 & 107 & 107 & 0 & 0 & 107 & 107 & 0 & 0 \\
\hline sfjs03 & 221 & 221 & 0 & 0,01 & 221 & 221 & 0 & 0,01 & 221 & 221 & 0 & 0,04 \\
\hline sfjs04 & 355 & 355 & 0 & 0,03 & 355 & 355 & 0 & 0,01 & 355 & 355 & 0 & 0,02 \\
\hline sfjs05 & 119 & 119 & 0 & 0,10 & 119 & 119 & 0 & 0,01 & 119 & 119 & 0 & 0,02 \\
\hline sfjs06 & 320 & 320 & 0 & 0,03 & 320 & 320 & 0 & 0,01 & 320 & 320 & 0 & 0,02 \\
\hline sfjs 07 & 397 & 397 & 0 & 0 & 397 & 397 & 0 & 0,01 & 397 & 397 & 0 & 0 \\
\hline sfjs08 & 253 & 253 & 0 & 0,02 & 253 & 253 & 0 & 0,01 & 253 & 253 & 0 & 0,01 \\
\hline sfjs09 & 210 & 210 & 0 & 0,03 & 210 & 210 & 0 & 0,01 & 210 & 210 & 0 & 0,01 \\
\hline sfjs10 & 516 & 516 & 0 & 0,15 & 516 & 516 & 0 & 0,01 & 516 & 516 & 0 & 0,01 \\
\hline mfjs01 & 468 & 468 & 0 & 0,32 & 468 & 468 & 0 & 0,47 & 468 & 468 & 0 & 0,30 \\
\hline mfjs02 & 446 & 446 & 0 & 0,37 & 446 & 446 & 0 & 0,66 & 446 & 446 & 0 & 0,72 \\
\hline mfjs03 & 466 & 466 & 0 & 0,78 & 466 & 466 & 0 & 1,60 & 466 & 466 & 0 & 1,44 \\
\hline mfjs04 & 554 & 554 & 0 & 2,33 & 554 & 554 & 0 & 4,47 & 554 & 554 & 0 & 3,43 \\
\hline mfjs 05 & 514 & 514 & 0 & 1,02 & 514 & 514 & 0 & 0,81 & 514 & 514 & 0 & 0,80 \\
\hline mfjs06 & 634 & 634 & 0 & 4,23 & 634 & 634 & 0 & 2,81 & 634 & 634 & 0 & 1,87 \\
\hline mfjs07 & 879 & 879 & 0 & 36,62 & 879 & 879 & 0 & 19,73 & 879 & 879 & 0 & 13,50 \\
\hline mfjs08 & 884 & 884 & 0 & 248,41 & 884 & 884 & 0 & 150,34 & 884 & 884 & 0 & 151,50 \\
\hline mfjs09 & 866,21 & 1070 & 19,04 & 3600 & 921 & 1084 & 15,04 & 3600 & 858 & 1071 & 19,93 & 3600 \\
\hline mfjs10 & 1009 & 1208 & 16,47 & 3600 & 1021 & 1237 & 17,46 & 3600 & 1008 & 1229 & 17,98 & 3600 \\
\hline
\end{tabular}

cálculo das médias do gap, foram consideradas, para cada conjunto, as instâncias em que foram obtidos os limitantes superiores e inferiores em ambas as abordagens. Dessa forma, as instâncias 09a, 14a, 15a e 18a não fazem parte do cálculo da média do gap para o conjunto DPdata.

Ao compararmos os dados MILP-1-Gurobi e MILP-2-Gurobi na Tabela 23, observamos que, mesmo tendo uma quantidade superior de variáveis binárias e de restrições, a média do gap e a média do tempo de processamento foram inferiores em MILP-2-Gurobi nos conjuntos Fdata e BCdata. Nestes dois conjuntos, a solução ótima para a maioria das instâncias foi obtida em menos de uma hora de processamento. Ao considerarmos os conjuntos de maior porte, isto é, BRdata e DPdata, a média do gap e a média do tempo de processamento foi superior em MILP-2-Gurobi. No conjunto DPdata, ao contrário de MILP-1-Gurobi, o procedimento MILP-2-Gurobi conseguiu encontrar uma solução factível para as instâncias 09a e 18a. Nestes dois problemas, dentro do limite de tempo, não houve exploração dos nós na árvore de busca e a solução factível foi obtida através de métodos heurísticos definidos pelo resolvedor. 
Tabela 20 - Resultados computacionais de MILP-1-Gurobi, MILP-2-Gurobi e MILP-2-B\&C para o conjunto BRdata.

\begin{tabular}{|c|c|c|c|c|c|c|c|c|c|c|c|c|}
\hline \multirow[t]{2}{*}{ Problema } & \multicolumn{4}{|c|}{ MILP-1-Gurobi } & \multicolumn{4}{|c|}{ MILP-2-Gurobi } & \multicolumn{4}{|c|}{ MILP-2-B\&C } \\
\hline & $\mathrm{LB}$ & $C_{\text {Max }}$ & $\begin{array}{l}\text { Gap } \\
(\%)\end{array}$ & $\begin{array}{l}\text { Tempo } \\
\text { (s) }\end{array}$ & $\mathrm{LB}$ & $C_{\text {Max }}$ & $\begin{array}{l}\text { Gap } \\
(\%)\end{array}$ & $\begin{array}{l}\text { Tempo } \\
\text { (s) }\end{array}$ & $\mathrm{LB}$ & $C_{\operatorname{Max}}$ & $\begin{array}{l}\text { Gap } \\
(\%)\end{array}$ & $\begin{array}{l}\text { Tempo } \\
\text { (s) }\end{array}$ \\
\hline MK01 & 40 & 40 & 0 & 87,29 & 40 & 40 & 0 & 412,77 & 40 & 40 & 0 & 45,41 \\
\hline MK02 & 25 & 27 & 7,41 & 3600 & 25 & 27 & 7,41 & 3600 & 25 & 27 & 7,41 & 3600 \\
\hline MK03 & 100 & 204 & 50,98 & 3600 & 94 & 215 & 56,28 & 3600 & 204 & 204 & 0 & 64,90 \\
\hline MK04 & 41 & 60 & 31,67 & 3600 & 40 & 66 & 39,39 & 3600 & 60 & 60 & 0 & 1458,11 \\
\hline MK05 & 65 & 178 & 63,48 & 3600 & 62,99 & 203 & 68,97 & 3600 & 138,58 & 192 & 27,82 & 3600 \\
\hline MK06 & 38,22 & 66 & 42,08 & 3600 & 37 & 76 & 51,31 & 3600 & 39,63 & 64 & 38,08 & 3600 \\
\hline MK07 & 61 & 152 & 59,87 & 3600 & 80 & 172 & 53,49 & 3600 & 133 & 154 & 13,64 & 3600 \\
\hline MK08 & 177,51 & 523 & 66,06 & 3600 & 177 & 533 & 66,79 & 3600 & 523 & 523 & 0 & 40,50 \\
\hline MK09 & 150,12 & 329 & 54,37 & 3600 & 146 & 398 & 63,32 & 3600 & 307 & 353 & 13,03 & 3600 \\
\hline MK10 & 119 & 262 & 54,58 & 3600 & 119 & 296 & 59,80 & 3600 & 181 & 280 & 35,36 & 3600 \\
\hline
\end{tabular}

Em relação ao nosso algoritmo B\&C, a Tabela 23 deixa evidente que a inclusão dos planos de corte propostos neste trabalho reduziu drasticamente a quantidade média de nós explorados pelo algoritmo MILP-2-B\&C. Além disso, com exceção do conjunto DPdata, o tempo de processamento também foi reduzido. Pelo fato de que, no conjunto DPdata, a solução ótima não foi obtida para nenhuma das instâncias dentro do limite de tempo, o tempo médio é de 3600 segundos. A média dos gaps também foi reduzida nos conjuntos BRdata, BCdata e DPBCdata. A redução mais significativa foi obtida no conjunto BRdata, sendo 43,04\% em MILP-1-Gurobi e 13,53\% em MILP-2-B\&C. Como MILP-2-B\&C não reduziu o gap nas instâncias mfjs09 e mfjs10, e estas são as únicas instâncias do conjunto em que a solução ótima não foi obtida, a média dos gaps foi melhor em MILP-1-Gurobi.

Conforme comentado anteriormente, o processo de encontrar inequações violadas pode requerer um tempo de processamento elevado. Se o algoritmo de separação consome muito tempo em relação ao benefício obtido, talvez não seja interessante incluí-lo em um algoritmo B\&C. A Tabela 24 apresenta a porcentagem média do tempo utilizado pela etapa de separação em relação ao tempo de processamento (coluna Tempo Separ. (\%)) e a média de inequações violadas (coluna Inequações Violadas). De acordo com a Tabela 24, considerando os conjuntos BRdata, BCdata e DPdata, o tempo de separação representou, na abordagem MILP-2-B\&C, no máximo 0,64\% do tempo total. Entretanto, para o conjunto Fdata, essa etapa correspondeu a 8,11\% do tempo total de processamento. A discrepância do valor da porcentagem em relação aos demais conjuntos ocorreu pelo fato de que o conjunto Fdata é de pequeno porte, sendo que, nas 16 primeiras instâncias (sfjs01-sfjs10 e mfjs01-mfjs06), a solução ótima é obtida em menos de 3,5 segundos. Dessa forma, um baixo valor no tempo de separação representa um proporção alta em relação ao tempo total. Por exemplo, a solução ótima para a instância sfjs10 é obtida em 0,012 segundo e o tempo de separação é igual a 0,005 segundo. Assim, o tempo gasto equivale a 41,66\% do tempo total de processamento. Considerando as instâncias mais significativas, isto é, mfjs07-mfjs10, a média do tempo de separação é igual a $0,06 \%$. Acreditamos que os valores obtidos indicam que o tempo gasto pelos algoritmos de separação não tem influência no tempo total e que não há necessidade 
Tabela 21 - Resultados computacionais de MILP-1-Gurobi, MILP-2-Gurobi e MILP-2-B\&C para o conjunto BCdata.

\begin{tabular}{|c|c|c|c|c|c|c|c|c|c|c|c|c|}
\hline \multirow[t]{2}{*}{ Problema } & \multicolumn{4}{|c|}{ MILP-1-Gurobi } & \multicolumn{4}{|c|}{ MILP-2-Gurobi } & \multicolumn{4}{|c|}{ MILP-2-B\&C } \\
\hline & LB & $C_{\text {Max }}$ & $\begin{array}{l}\text { Gap } \\
(\%)\end{array}$ & $\begin{array}{l}\text { Tempo } \\
(\mathrm{s})\end{array}$ & $\mathrm{LB}$ & $C_{\text {Max }}$ & $\begin{array}{l}\text { Gap } \\
(\%)\end{array}$ & $\begin{array}{l}\text { Tempo } \\
\text { (s) }\end{array}$ & $\mathrm{LB}$ & $C_{M a x}$ & $\begin{array}{l}\text { Gap } \\
(\%)\end{array}$ & $\begin{array}{l}\text { Tempo } \\
(\mathrm{s})\end{array}$ \\
\hline $\mathrm{mt10c1}$ & 927 & 927 & 0 & 67,79 & 927 & 927 & 0 & 158,93 & 927 & 927 & 0 & 175,25 \\
\hline $\mathrm{mt10cc}$ & 908 & 908 & 0 & 41,08 & 908 & 908 & 0 & 124,85 & 908 & 908 & 0 & 68,94 \\
\hline mt10x & 918 & 918 & 0 & 116,17 & 918 & 918 & 0 & 271,39 & 918 & 918 & 0 & 170,50 \\
\hline $\operatorname{mt10xx}$ & 918 & 918 & 0 & 83,57 & 918 & 918 & 0 & 81,55 & 918 & 918 & 0 & 183,63 \\
\hline $\operatorname{mt10xxx}$ & 918 & 918 & 0 & 126,79 & 918 & 918 & 0 & 121,38 & 918 & 918 & 0 & 139,11 \\
\hline mt10xy & 905 & 905 & 0 & 39,22 & 905 & 905 & 0 & 60,13 & 905 & 905 & 0 & 95,56 \\
\hline mt10xyz & 847 & 847 & 0 & 20,91 & 847 & 847 & 0 & 51,23 & 847 & 847 & 0 & 27,19 \\
\hline setb4c9 & 914 & 914 & 0 & 870,67 & 914 & 914 & 0 & 418,14 & 914 & 914 & 0 & 520,97 \\
\hline setb4cc & 907 & 907 & 0 & 3392,31 & 907 & 907 & 0 & 838,28 & 907 & 907 & 0 & 482,41 \\
\hline setb4x & 892 & 925 & 3,56 & 3600 & 887 & 929 & 4,52 & 3600 & 925 & 925 & 0 & 711,61 \\
\hline setb4xx & 925 & 925 & 0 & 3092,15 & 925 & 925 & 0 & 2348,50 & 925 & 925 & 0 & 2235,41 \\
\hline setb4xxx & 888 & 925 & 3,99 & 3600 & 887 & 925 & 4,11 & 3600 & 925 & 925 & 0 & 1272,64 \\
\hline setb4xy & 848 & 910 & 6,81 & 3600 & 893 & 910 & 1,87 & 3488,85 & 901,25 & 910 & 0,96 & 3600 \\
\hline setb4xyz & 902 & 902 & 0 & 3475,69 & 902 & 902 & 0 & 345,99 & 902 & 902 & 0 & 393,60 \\
\hline seti5c12 & 1043 & 1199 & 13,01 & 3600 & 1061 & 1184 & 10,38 & 3600 & 1169 & 1169 & 0 & 3478,43 \\
\hline seti5cc & 1087 & 1140 & 4,65 & 3600 & 1088 & 1137 & 4,31 & 3600 & 1079 & 1138 & 5,18 & 3600 \\
\hline seti5x & 1024 & 1203 & 14,88 & 3600 & 1041 & 1205 & 13,61 & 3600 & 1192 & 1200 & 0,67 & 3600 \\
\hline seti5xx & 1104 & 1204 & 8,30 & 3600 & 1070 & 1200 & 10,83 & 3600 & 1192 & 1194 & 0,17 & 3600 \\
\hline seti5xxx & 1049 & 1204 & 12,87 & 3600 & 1059 & 1204 & 12,04 & 3600 & 1192 & 1194 & 0,17 & 3600 \\
\hline seti5xy & 1086 & 1140 & 4,73 & 3600 & 1088 & 1137 & 4,31 & 3600 & 1079 & 1138 & 5,18 & 3600 \\
\hline seti5xyz & 1045 & 1138 & 8,17 & 3600 & 1051 & 1128 & 6,83 & 3600 & 1055 & 1128 & 6,47 & 3600 \\
\hline
\end{tabular}

de utilizar outras abordagens, como as descritas na seção 1.4.3.

Com o intuito de verificar se a combinação das inequações dos grupos G1 e G2 com os cortes disponíveis no Gurobi são eficientes para o JSF, implementamos um novo algoritmo $\mathrm{B} \& \mathrm{C}$ contendo os 13 cortes do resolvedor e as inequações (3.32), (3.34), (3.33) e (3.35). Denominamos esse procedimento de MILP-2-B\&C+CG. Os parâmetros $k$ e $f$ foram fixados em 300 e 1500, respectivamente. Os resultados dos experimentos estão apresentados na Tabela 25.

Como indicado na Tabela 25, a diferença entre as médias dos gaps nas duas abordagens é pequena. Enquanto MILP-2-B\&C obteve a melhor média dos gaps nos conjuntos BRdata e BCdata, a abordagem MILP-2-B\&C+CG obteve melhores resultados em Fdata e DPdata. A diferença entre as médias dos tempos de processamento também foi pequena. A diferença mais significativa foi em relação ao número médio de nós explorados no conjunto Fdata. O valor obtido em MILP-2-B\&C+CG é superior ao dobro de MILP-2-B\&C. Embora não 
Tabela 22 - Resultados computacionais de MILP-1-Gurobi, MILP-2-Gurobi e MILP-2-B\&C para o conjunto DPdata.

\begin{tabular}{|c|c|c|c|c|c|c|c|c|c|c|c|c|}
\hline \multirow[t]{2}{*}{ Problema } & \multicolumn{4}{|c|}{ MILP-1-Gurobi } & \multicolumn{4}{|c|}{ MILP-2-Gurobi } & \multicolumn{4}{|c|}{ MILP-2-B\&C } \\
\hline & LB & $C_{\text {Max }}$ & $\begin{array}{l}\text { Gap } \\
(\%)\end{array}$ & $\begin{array}{l}\text { Tempo } \\
\text { (s) }\end{array}$ & LB & $C_{\operatorname{Max}}$ & $\begin{array}{l}\text { Gap } \\
(\%)\end{array}$ & $\begin{array}{l}\text { Tempo } \\
\text { (s) }\end{array}$ & LB & $C_{M a x}$ & $\begin{array}{l}\text { Gap } \\
(\%)\end{array}$ & $\begin{array}{l}\text { Tempo } \\
\text { (s) }\end{array}$ \\
\hline $01 \mathrm{a}$ & 1477 & 2783 & 46,93 & 3600 & 1518 & 2865 & 47,01 & 3600 & 2505 & 2530 & 0,99 & 3600 \\
\hline $02 \mathrm{a}$ & 1433 & 2584 & 44,54 & 3600 & 1433 & 2495 & 42,56 & 3600 & 1628 & 2509 & 35,11 & 3600 \\
\hline $03 \mathrm{a}$ & 1392 & 2650 & 47,47 & 3600 & 1392 & 2463 & 43,48 & 3600 & 1392 & 2604 & 46,54 & 3600 \\
\hline $04 \mathrm{a}$ & 1452,02 & 2731 & 46,83 & 3600 & 1450 & 2930 & 50,53 & 3600 & 2503 & 2506 & 0,12 & 3600 \\
\hline $05 \mathrm{a}$ & 1389 & 2502 & 44,48 & 3600 & 1388 & 2642 & 47,46 & 3600 & 1546 & 2503 & 38,23 & 3600 \\
\hline $06 \mathrm{a}$ & 1343,92 & 2390 & 43,77 & 3600 & 1339 & 2588 & 48,26 & 3600 & 1339 & 2530 & 47,08 & 3600 \\
\hline $07 \mathrm{a}$ & 1465 & 2674 & 45,21 & 3600 & 1494 & 2752 & 45,71 & 3600 & 2206 & 2465 & 10,51 & 3600 \\
\hline $08 \mathrm{a}$ & 1400 & 2290 & 38,86 & 3600 & 1400 & 2685 & 47,88 & 3600 & 1400 & 2698 & 48,11 & 3600 \\
\hline $09 a$ & 1400 & - & - & 3600 & 1400 & 2467 & 43,25 & 3600 & 1400 & 2847 & 50,83 & 3600 \\
\hline $10 \mathrm{a}$ & 1436 & 2589 & 44,53 & 3600 & 1503 & 3033 & 50,44 & 3600 & 2197 & 2425 & 9,40 & 3600 \\
\hline $11 \mathrm{a}$ & 1338 & 2324 & 42,43 & 3600 & 1337 & 2518 & 46,90 & 3600 & 1337 & 2418 & 44,71 & 3600 \\
\hline $12 \mathrm{a}$ & 1310 & 2362 & 44,54 & 3600 & 1310 & 13738 & 90,46 & 3600 & 1310 & 2559 & 48,81 & 3600 \\
\hline $13 \mathrm{a}$ & 1432,21 & 2876 & 50,20 & 3600 & 1429 & 2933 & 51,28 & 3600 & 2100 & 2804 & 25,11 & 3600 \\
\hline $14 \mathrm{a}$ & 1354 & 2561 & 47,13 & 3600 & 1354 & - & - & 3600 & 1354 & 2884 & 53,05 & 3600 \\
\hline $15 \mathrm{a}$ & 1354 & - & - & 3600 & 1354 & - & - & 3600 & 1354 & - & - & 3600 \\
\hline $16 \mathrm{a}$ & 1412 & 2739 & 48,45 & 3600 & 1420,13 & 2793 & 49,15 & 3600 & 2100 & 2914 & 27,93 & 3600 \\
\hline $17 \mathrm{a}$ & 1291 & 2707 & 52,31 & 3600 & 1291 & 17949 & 92,80 & 3600 & 1291 & 10270 & 87,43 & 3600 \\
\hline $18 \mathrm{a}$ & 1289 & - & - & 3600 & 1289 & 17560 & 92,66 & 3600 & 1289 & 17560 & 92,66 & 3600 \\
\hline
\end{tabular}

Tabela 23 - Resumo dos resultados para cada conjunto de instâncias teste. Os valores representam a média para cada conjunto.

\begin{tabular}{|c|c|c|c|c|c|c|c|c|c|}
\hline \multirow[t]{2}{*}{ Conjunto } & \multicolumn{3}{|c|}{ MILP-1-Gurobi } & \multicolumn{3}{|c|}{ MILP-2-Gurobi } & \multicolumn{3}{|c|}{ MILP-2-B\&C } \\
\hline & Nós & $\begin{array}{l}\text { Gap } \\
(\%)\end{array}$ & $\begin{array}{l}\text { Tempo } \\
\text { (s) }\end{array}$ & Nós & $\begin{array}{l}\text { Gap } \\
(\%)\end{array}$ & $\begin{array}{l}\text { Tempo } \\
\text { (s) }\end{array}$ & Nós & $\begin{array}{l}\text { Gap } \\
(\%)\end{array}$ & $\begin{array}{l}\text { Tempo } \\
\text { (s) }\end{array}$ \\
\hline Fdata & $116.088,35$ & 1,78 & 374,71 & $141.427,3$ & 1,68 & 370,16 & 9.957 & 1,90 & 368,69 \\
\hline BRdata & $103.757,20$ & 43,05 & $3.248,73$ & $42.706,30$ & 46,68 & $3.281,28$ & $7.576,60$ & 13,53 & $2.322,91$ \\
\hline BCdata & $1.094 .863,86$ & 3,86 & $2.250,65$ & $738.688,48$ & 3,47 & $1.943,83$ & $137.140,76$ & 0,90 & $1.674,08$ \\
\hline DPdata & $33.086,64$ & 45,75 & 3.600 & $54.070,50$ & 53,85 & 3.600 & $4.791,50$ & 33,58 & 3.600 \\
\hline
\end{tabular}

esteja indicado na Tabela 25, MILP-2-B\&C+CG provou a otimalidade para duas novas instâncias: setb4xy e seti5xx do conjunto DPdata. Mas duas soluções ótimas obtidas por MILP-2-B\&C, setb4xx e seti5c12, deixaram de ter o gap nulo em MILP-2-B\&C+CG. Ainda, MILP-2-B\&C+CG obteve um limitante superior para a instância 15a, mas não para a instância 14a do conjunto DPdata. No cálculo da média dos gap, não foram consideradas as 
Tabela 24 - Média das inequações violadas e da porcentagem destinada ao tempo da etapa de separação de MILP-2-B\&C.

\begin{tabular}{|c|c|c|}
\hline \multirow[t]{2}{*}{ Conjunto } & \multicolumn{2}{|c|}{ MILP-2-B\&C } \\
\hline & Tempo Separ. (\%) & Inequações Violadas \\
\hline Fdata & 8,11 & 1003,7 \\
\hline BRdata & 0,64 & 3608,1 \\
\hline BCdata & 0,63 & 4009 \\
\hline DPdata & 0,44 & 2988,22 \\
\hline
\end{tabular}

Tabela 25 - Resumo dos resultados para cada conjunto de instâncias teste. Os valores representam a média para cada conjunto.

\begin{tabular}{|c|c|c|c|c|c|c|}
\hline \multirow[t]{2}{*}{ Conjunto } & \multicolumn{3}{|c|}{ MILP-2-B\&C } & \multicolumn{3}{|c|}{ MILP-2-B\&C+CG } \\
\hline & Nós & Gap (\%) & Tempo (s) & Nós & Gap (\%) & Tempo (s) \\
\hline Fdata & 9.957 & 1,90 & 368,69 & $19.753,85$ & 1.84 & 370,35 \\
\hline BRdata & $7.576,60$ & 13,53 & $2.322,91$ & $7.231,00$ & 13.96 & $2.244,08$ \\
\hline BCdata & $137.140,76$ & 0,90 & $1.674,08$ & $172.729,29$ & 0.80 & $1.656,70$ \\
\hline DPdata & $4.791,50$ & 38,35 & 3.600 & $3.488,17$ & 38.71 & 3.600 \\
\hline
\end{tabular}

instâncias 14a e 15a. Assim, acreditamos que os cortes disponíveis no Gurobi podem favorecer a obter novas soluções ótimas para o JSF e que a abordagem MILP-2-B\&C+CG pode ser utilizada como um procedimento adicional ao MILP-2-B\&C.

\subsubsection{Instâncias em aberto}

Em decorrência da complexidade do JSF, ainda há muitas instâncias em aberto. Conforme citado anteriormente, os conjuntos BRdata e DPdata apresentam 11 problemas em que não foi possível demonstrar ou obter a solução ótima.

Além dos conjuntos de instâncias teste utilizados neste capítulo, outro conjunto utilizado na literatura é o proposto por Hurink, Jurisch e Thole (1994). Este conjunto é constituído por 129 instâncias criadas a partir de 43 instâncias do JS (conjunto Sdata). Conforme a média do número de máquinas habilitadas a processar cada operação, os problemas são divididos em três grupos: Edata, Rdata e Vdata. O número de tarefas varia entre 6 e 30, o número de máquinas entre 5 e 15 e o número de operações para todas as tarefas entre 36 e 300. Os três conjuntos apresentam 16 instâncias em aberto, sendo 2 em Edata, 12 em Rdata e 2 em Vdata. Não utilizamos as intâncias de Hurink, Jurisch e Thole (1994) pois acreditamos que as utilizadas nesta tese contemplem os casos de pequeno, médio e grande porte.

Com exceção das instâncias do conjunto Fdata, os valores dos limitantes para cada instância dos conjuntos BRdata, BCdata, DPdata, Edata, Rdata, Vdata e Sdata estão dispo- 
níveis no sítio http://www.quintiq.com/optimization/flexible-job-shop-scheduling-problemresults.html. Este endereço é mantido pela empresa Quintiq (QUINTIQ, 2016), de origem holandesa e pertencente à Dassault Systèmes. A Quintiq desenvolve um software de otimização destinado a problemas de programação de tarefas e cadeia de suprimentos. Além do JSF, também é possível obter dados de instâncias para o problema de Roteamento de Veículos com Janela de Tempo e para o problema de Coleta e Entrega com Janela de Tempo. Para cada conjunto Edata, Rdata e Vdata, além das instâncias originais, são acrescentados 23 novos problemas. Dessa forma, em Results-Quintiq (2016) estão descritos os resultados de 303 instâncias para o JSF. Desse total, foi provada a otimalidade da solução em 275.

As principais abordagens que obtiveram pela primeira vez o limitante superior ótimo e, para os problemas em aberto, o melhor limitante superior, estão distribuídas entre as técnicas de busca local de Mastrolilli e Gambardella (2000), de programação por restríções de Schutt, Feydy e Stuckey (2013) e o uso dos resolvedores Quintiq (QUINTIQ, 2016) e CPLEX CP Optimizer (CPLEX-CP, 2016). As técnicas citadas também são responsáveis para os melhores resultados do limitante inferior.

Aplicamos o algoritmo MILP-2-B\&C nas instâncias em aberto, utilizando a configuração adotada na seção anterior e os resultados obtidos estão descritos na Tabela 26. As colunas "Melhor LB" e "Melhor UB" indicam os valores dos melhores limitantes inferior e superior, respectivamente, encontrados na literatura, "MILP-2-Gurobi LB" e "MILP-2-Gurobi UB" representam os limitantes obtidos por MILP-2-B\&C após 24 horas de processamento. Os resultados mostram que o algoritmo MILP-2-B\&C não melhorou os valores dos limitantes em nenhum dos problemas em aberto.

\subsection{Resumo}

Neste capítulo, apresentamos 8 classes de inequações válidas para o JSF, sendo 2 classes obtidas do POL e as demais classes adaptadas do JS. Em decorrência das classes de inequações derivadas do JS, o melhor modelo encontrado na literatura foi reformulado para atender a restrição do tipo (3.40). Caso contrário, a solução ótima de MILP-1 representará apenas um limitante superior para o makespan ótimo.

Inicialmente as classes de inequações foram avaliadas em um algoritmo de $\mathrm{PC}$, e as que tornaram a relaxação do modelo "mais justa", foram utilizadas em um algoritmo B\&C. Os experimentos computacionais mostraram que nosso algoritmo B\&C melhorou a qualidade das soluções e reduziu drasticamente a quantidade média de nós explorados. Com exceção do conjunto DPdata, o tempo de processamento também foi reduzido. Entretanto, considerando as instâncias em aberto da literatura, nosso algoritmo não encontrou novas soluções ótimas e também não melhorou os valores dos limitantes. 
Tabela 26 - Resultados computacionais de MILP-2-B\&C para as instâncias em aberto.

\begin{tabular}{|c|c|c|c|c|}
\hline Instância & Melhor LB & Melhor UB & MILP-2-Gurobi LB & MILP-2-Gurobi \\
\hline Mk10 & 189 & 193 & 181 & 225 \\
\hline $05 \mathrm{a}$ & 2192 & 2204 & 1556 & 2341 \\
\hline $06 \mathrm{a}$ & 2163 & 2171 & 1339 & 2406 \\
\hline $07 \mathrm{a}$ & 2216 & 2264 & 2206 & 2455 \\
\hline $10 \mathrm{a}$ & 2212 & 2241 & 2197 & 2349 \\
\hline $11 \mathrm{a}$ & 2018 & 2037 & 1337 & 2491 \\
\hline $12 \mathrm{a}$ & 1969 & 1984 & 1310 & 2325 \\
\hline $13 \mathrm{a}$ & 2197 & 2239 & 2100 & 2487 \\
\hline $16 \mathrm{a}$ & 2193 & 2231 & 2091,11 & 2521 \\
\hline $17 \mathrm{a}$ & 2088 & 2105 & 1291 & 2758 \\
\hline $18 \mathrm{a}$ & 2057 & 2070 & 1289 & 17527 \\
\hline edata-abz7 & 604 & 610 & 550,50 & 621 \\
\hline edata-abz8 & 625 & 636 & 578 & 646 \\
\hline rdata-abz7 & 493 & 524 & 441 & 579 \\
\hline rdata-abz8 & 507 & 536 & 449 & 588 \\
\hline rdata-abz9 & 517 & 536 & 467 & 578 \\
\hline rdata-la21 & 808 & 825 & 717 & 863 \\
\hline rdata-la22 & 741 & 755 & 664 & 790 \\
\hline rdata-la23 & 816 & 832 & 640 & 861 \\
\hline rdata-la24 & 775 & 796 & 704 & 817 \\
\hline rdata-la25 & 768 & 783 & 736 & 799 \\
\hline rdata-la26 & 1056 & 1057 & 717 & 1114 \\
\hline rdata-la28 & 1075 & 1076 & 756 & 1141 \\
\hline rdata-la29 & 993 & 994 & 723 & 1021 \\
\hline rdata-la30 & 1068 & 1071 & 774 & 1121 \\
\hline sdata-abz8 & 653 & 667 & 597 & 674 \\
\hline
\end{tabular}





\section{Capítulo 4 Matheuristics}

Em problemas de otimização de grande porte, as metaheurísticas são abordagens que permitem produzir boas soluções e de forma rápida. Entretanto, não é possível provar a otimalidade de uma solução, tarefa esta de responsabilidade de métodos exatos. Com o intuito de aproveitar as melhores características de cada uma das abordagens, encontrase uma técnica de otimização denominada de matheuristics. Matheuristics são algoritmos de otimização obtidos pela combinação de (meta)heurísticas e técnicas de programação matemática.

Neste capítulo, apresentamos duas abordagens híbridas utilizadas na resolução do JSF. Estas abordagens combinam duas estratégias bem conhecidas: MILP e busca local. A primeira é denominada de local branching e a segunda de diversification, refining and tight-refining. Tais procedimentos procuram aproveitar tanto o potencial dos resolvedores de MILPs quanto as características de metaheurísticas. Além disso, em cada abordagem descrevemos os resultados obtidos pelos experimentos computacionais.

\subsection{Introdução}

As abordagens utilizadas para resolver COPs podem ser divididas em duas categorias: métodos exatos e métodos aproximados. Dentre as abordagens exatas, destacamos a programação dinâmica e os métodos baseados em relaxações, tais como algoritmo de PC e geração de colunas, $\mathrm{B} \& \mathrm{~B}, \mathrm{~B} \& \mathrm{C}$, branch and price e branch and cut and price. Os métodos aproximados são divididos em algoritmos aproximados e algoritmos heurísticos.

Os métodos exatos e de aproximação, embora tenham as suas vantagens e desvantagens, podem ser vistos como procedimentos complementares. Portanto, uma ideia natural é tentar combinar essas duas técnicas em um algoritmo mais poderoso, de característica híbrida. O método resultante pode ser de natureza heurística ou exata.

Embora esta ideia não seja nova, as técnicas que procuraram combinar a abordagem exata com (meta)heurística para resolver COP se tornaram populares nas três últimas décadas. Eventos científicos internacionais como o Workshop on Hybrid Metaheuristics, realizado desde 2004, e o Matheuristics-International Workshop on Model-based Metaheuristics, realizado a cada dois anos desde 2006, e as duas edições do Handbook of Metaheuristics (GLOVER; KOCHENBERGER, 2006; GENDREAU; POTVIN, 2010) enfatizam o sucesso e a importância dessa linha de pesquisa. O termo matheuristics foi estabelecido no primeiro Workshop on Hybrid Metaheuristics para referir a combinações de metaheurísticas e métodos de programação matemática (PUCHINGER; RAIDL, 2008). Outro termo utilizado na literatura para esta linha 
de pesquisa é modelos baseados em metaheurísticas.

No trabalho de Dumitrescu e Stützle (2003), os autores descrevem as vantagens e as desvantagens de combinar as abordagens exatas e heurísticas em COP. Os autores focam a atenção nas abordagens em que a técnica de busca local é fortalecida pelo uso de algoritmos exatos e revisam alguns trabalhos da literatura em que novos algoritmos foram criados a partir destas combinações. Ainda nas abordagens que utilizam a busca local com métodos exatos, Fernandes e Lourenço (2007) propõem o termo Optimized Search Heuristics para descrever tal abordagem e uma nova classificação quanto ao seu uso. Fernandes e Lourenço também apresentam uma listagem de sua utilização em uma grande quantidade de problemas na literatura, e o JS aparece como sendo o problema com o maior número de aplicações.

Um dos primeiros trabalhos que abordam, de forma geral, as técnicas de matheuristics para resolver COP foi proposto por Puchinger e Raidl (2005). Conforme a combinação dos métodos exatos e das metaheurísticas, as abordagens foram classificadas em duas principais categorias: colaborativa e combinação integrativa. Na primeira classificação, embora os métodos exatos e metaheurísticos sejam independentes, há troca de informações entre ambos. Os dois métodos podem ser executados de forma sequencial, paralela ou interligada. Na forma sequencial, ou o método exato é executado como uma ferramenta de pré-processamento que antecede a metaheurística, ou o contrário. Os autores descrevem vários trabalhos da literatura, tais como a combinação da técnica de geração de colunas com a heurística squeaky wheel optimization em Clements et al. (1997), o algoritmo genético com programação inteira em Klau et al. (2004), o algoritmo genético com MILP em Lin, Bean e White (2004) e o B\&B com o algoritmo genético em Nagar, Heragu e Haddock (1995). Na forma paralela ou interligada, as duas técnicas são executadas de forma simultânea, seja em paralelo, isto é, funcionando ao mesmo tempo em diferentes processadores, ou de uma maneira interligada, alternando entre os dois algoritmos. Puchinger e Raidl relatam que o uso da abordagem paralela ou interligada é menos frequente. Na combinação integrativa, um método é incorporado ou subordinado ao outro, de tal forma que o primeiro método atua como um regente, e o segundo, como um subordinado do primeiro. Dois casos são considerados. O primeiro consiste em incorporar algoritmos exatos em metaheurísticas. Um exemplo é o de utilizar métodos exatos para codificar as partes restantes de um cromossomo em algum algoritmo genético (veja em Staggemeier et al. (2002)). A segunda possibilidade de colaboração integrativa é incorporar metaheurísticas em métodos exatos. Por exemplo, metaheurísticas podem ser utilizadas como ferramentas no processo de gerar colunas ou planos de corte no algoritmo branch and cut and price, como no trabalho de Ribeiro e Lorena (2000), ou para determinar limitantes ou soluções factíveis para o método B\&B, como em Woodruff (1999).

O trabalho de Puchinger e Raidl (2008) apresenta um estudo de técnicas que combinam, especificamente, programação linear e programação inteira com metaheurísticas em COP e classificam as técnicas em dez categorias metodológicas.

Em Doerner e Schmid (2010), é proposta uma pesquisa com as recentes abordagens matheuristics para diferentes problemas de roteamento de veículos. Os autores observaram que tal abordagem é bastante promissora nesta categoria de problema e destacam alguns aspectos fundamentais para os estudos futuros. Ainda no âmbito de roteamento de veículos, Archetti e Speranza (2014) apresentam uma visão geral de ideias bem sucedidas de matheuristics para obter soluções de alta qualidade. Os autores propõem três classes de matheuristics: abordagens de decomposição, heurísticas de melhoria e abordagens baseadas em geração de colunas.

Na sequência, apresentamos as abordagens local branching e diversification, refining 
and tight-refining. Conforme a categorização proposta por Puchinger e Raidl (2005), esses dois métodos são classificados como combinação integrativa, pois algumas ideias presentes na busca local são incorporadas durante a resolução do MILP.

\subsection{Local branching}

A técnica local branching (LB), introduzida por Fischetti e Lodi (2003), é uma abordagem exata que combina as ideias de busca em vizinhanças com resolvedores de MILP. O conceito da busca local está presente pela introdução de inequações, ou cortes, denominados local branching. Nos estágios iniciais, o objetivo consiste em explorar regiões menores e de menor esforço computacional do que o problema original, procurando, de forma rápida, obter soluções de boa qualidade.

Para descrever esta técnica, utilizamos a notação presente em Fischetti e Lodi (2003). Considere o problema $(\mathrm{P})$ descrito por

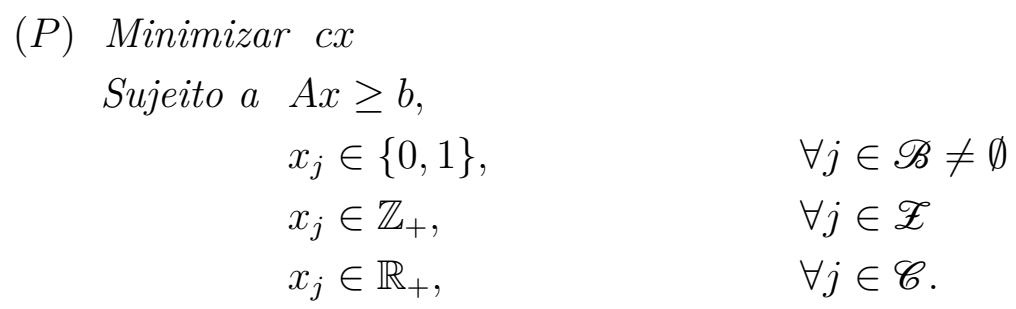

No problema $(\mathrm{P})$, o índice da variável $x$ é particionado em três conjuntos: $\mathscr{B}, \mathscr{Z}, \mathscr{C}$. O conjunto $\mathscr{B} \neq \emptyset$ contém os índices das variáveis binárias, enquanto os conjuntos $\mathscr{Z}$ e $\mathscr{C}$ contêm os possíveis índices para as variáveis inteiras e reais, respectivamente.

Dada uma solução factível $\bar{x}$ para o problema $(\mathrm{P})$, o conjunto denominado suporte binário de $\bar{x}$ é definido por

$$
\bar{S}=\left\{j \in \mathscr{B} ; \quad \bar{x}_{j}=1\right\}
$$

Para um dado parâmetro $k \in \mathbb{Z}^{+}$, a $k$-vizinhança $\Delta(x, \bar{x})$ de $\bar{x}$ representa o conjunto de soluções factíveis de (P) que satisfazem a restrição local branching definida por

$$
\Delta(x, \bar{x})=\sum_{j \in \bar{S}}\left(1-x_{j}\right)+\sum_{j \in \mathscr{B} \backslash \bar{S}} x_{j} \leq k .
$$

Na restrição (4.2), o primeiro somatório determina o número de variáveis que terão seus valores alterados na solução $\bar{x}$ de 1 para 0 , e no segundo somatório, de 0 para 1 . Logo, o acréscimo da $k$-vizinhança $\Delta(x, \bar{x})$ ao problema $(\mathrm{P})$ define o conjunto de soluções factíveis em que o número de variáveis binárias diferem de $\bar{x}$, no máximo, em $k$ valores.

Se a cardinalidade do suporte binário para qualquer solução factível de (P) é constante, a restrição local branching (4.2) é equivalente a

$$
\sum_{j \in \bar{S}}\left(1-x_{j}\right) \leq k^{\prime} \quad\left(k^{\prime}=k / 2\right)
$$


uma vez que, se $x_{j}$, com $j \in \bar{S}$, tem seu valor alterado de 1 para zero, uma outra variável binária com valor nulo deverá receber o valor unitário. Dessa forma, a troca sempre ocorrerá em um fator com multiplicidade 2 .

A restrição local branching pode ser utilizada dentro do método B\&B ou B\&C como uma estratégia de ramificação para explorar o espaço de soluções do problema (P). Assim, dada a solução incumbente $\bar{x}$, o espaço de soluções é particionado em dois conjuntos disjuntos pelo acréscimo das restrições $\Delta(x, \bar{x}) \leq k$ (ramificação à esquerda) e $\Delta(x, \bar{x}) \geq(k+1)$ (ramificação à direita).

A técnica procura explorar inicialmente os subproblemas, ou subMILPs, gerados pelos acréscimo da ramificação à esquerda. Como o parâmetro $k$ define o tamanho da vizinhança, a $k$-vizinhança $\Delta(x, \bar{x})$ correspondente ao nó esquerdo deve ser suficientemente pequena para ser otimizada em um curto tempo, mas grande o suficiente para conter soluções melhores que $\bar{x}$. Ainda, espera-se que o subproblema correspondente à ramificação esquerda seja mais fácil de ser resolvido do que o seu respectivo pai.

O procedimento tradicional do método LB está ilustrado na Figura 12. O método inicia-se a partir de uma solução factível $\bar{x}_{1}$ para o problema $(\mathrm{P})$ no nó raiz (nó 1$)$. Os nós esquerdo (nó 2) e direito (nó 3) são gerados pelo acréscimo das restrição $\Delta\left(x, \bar{x}_{1}\right) \leq k$ e $\Delta\left(x, \bar{x}_{1}\right) \geq(k+1)$, respectivamente, ao problema $(\mathrm{P})$, produzindo assim dois subproblemas. $\mathrm{O}$ quadrado em destaque e marcado pela letra "S" corresponde às subárvores exploradas por algum resolvedor de MILP. Se uma nova solução ótima $\bar{x}_{2}$, melhor que $\bar{x}_{1}$, for encontrada no nó 2 , então o nó à direita (nó 3) é ramificado pelo acréscimo das restrições $\Delta\left(x, \bar{x}_{2}\right) \leq k$ (nó 4) e $\Delta\left(x, \bar{x}_{2}\right) \geq(k+1)$ (nó 5$)$ e $\bar{x}_{2}$ se torna a nova solução incumbente. O processo se repete enquanto a solução ótima do subMILP à esquerda for melhor que a solução incumbente. Caso contrário, o último nó à direita é executado pelo resolvedor. Observe, na Figura 12, que a solução encontrada pelo resolvedor no nó 6 não atualizou a solução incumbente. Nesta situação, é adicionada somente a restrição $\Delta\left(x, \bar{x}_{3}\right) \geq(k+1)$ ao subproblema corrente e o nó 7 é explorado por completo.

Pretende-se, com esta estratégia, encontrar boas e rápidas soluções até o momento em que o método não traga mais benefícios, deixando o tempo maior de processamento para o estágio final, isto é, para a otimização do último nó à direita.

Visando aprimorar o comportamento do método, Fischetti e Lodi (2003) propõem duas estratégias de aperfeiçoamento que buscam alterar o tamanho da vizinhança $\Delta(x, \bar{x})$ conforme o tempo gasto na exploração do nó esquerdo. A primeira estratégia consiste em impor um limite de tempo para o processamento dos subMILPs e a segunda em atribuir um mecanismo de diversificação. Descrevemos, na sequência, o uso destas duas estratégias.

Devido ao parâmetro $k$, a solução ótima da ramificação à esquerda pode consumir muito tempo e, nesse caso, os autores aplicam um tempo limite para o processamento do nó. Se, dentro do limite de tempo, a solução incumbente for atualizada, o algoritmo retorna ao nó pai e cria, com base na nova solução incumbente, as novas ramificações à esquerda e à direita. Esta etapa está ilustrada na Figura 13. Observe que o nó 3 tem 3 filhos: nós 4, 4' e 5. No nó 4, embora a solução incumbente seja atualizada, o resolvedor finalizou o processamento em decorrência da imposição do limite de tempo. Dessa forma, uma nova ramificação foi criada com base na nova solução corrente $\bar{x}_{3}$, produzindo o subMILP no nó 4 '. Além disso, como a vizinhança associada ao nó 4 não foi explorada por completo, a restrição $\Delta\left(x, \bar{x}_{2}\right) \geq(k+1)$ não pode ser adicionada aos subproblemas associados aos nós 4' e 5 . Dado que a solução ótima no nó 4' representa a melhor obtida até o presente momento, o método continua em sua forma tradicional. 


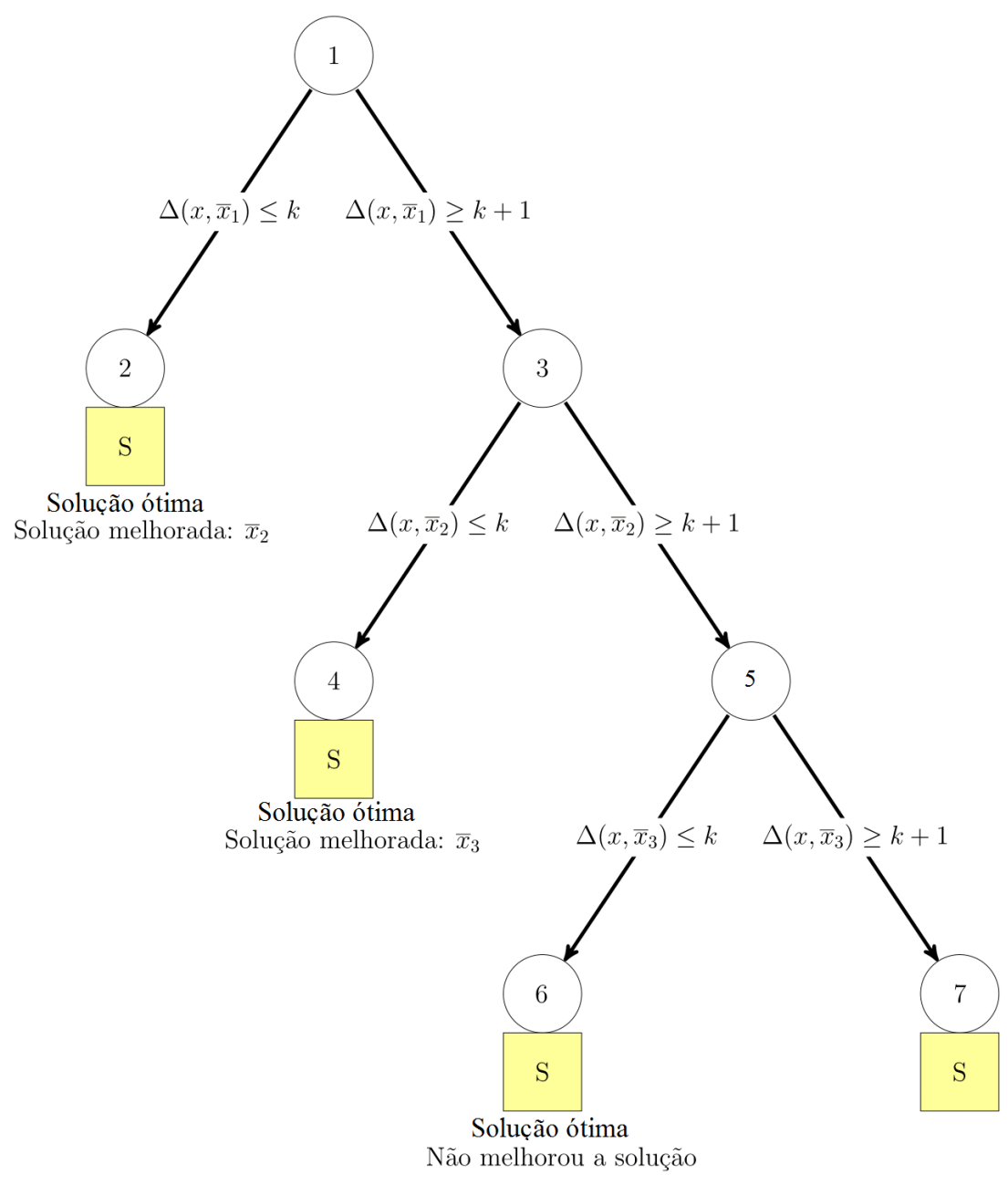

Figura 12 - Esquema do método LB tradicional.

Caso a solução não seja atualizada dentro do limite de tempo, o algoritmo retorna ao nó pai e o tamanho da vizinhança é reduzido pela metade. Dessa forma, busca-se intensificar a busca pela solução ótima dentro do limite de tempo, mas explorando uma vizinhança de menor tamanho. Esta etapa está representada pela Figura 14, em que o nó 3 tem 3 filhos. Como o resolvedor não melhorou a solução no nó 4 dentro do limite de tempo, o novo nó 4' é criado através da alteração do valor do parâmetro $k$ para $\left\lfloor\frac{k}{2}\right\rfloor$. Supõe-se que a solução ótima $\bar{x}_{3}$ encontrada no nó 4' é a melhor até o presente momento e, na sequência, o processamento ocorre de maneira usual. O processamento dos subproblemas relativos a cada nó esquerdo é finalizado no nó 6 , uma vez que a solução ótima obtida dentro do limite de tempo não é melhor que a solução corrente.

A segunda estratégia se refere a impor um mecanismo de diversificação ao método. A diversificação é uma importante etapa em toda metaheurística baseada em busca local. Esta etapa consiste em redirecionar a pesquisa para regiões ainda não suficientemente exploradas do espaço de soluções. No LB, a estratégia de diversificação é utilizada quando a solução do subproblema relativo ao nó esquerdo não for melhor que a solução atual. Nesse caso, objetivase adiar a utilização do resolvedor em obter a solução para o nó direito. Para possibilitar a exploração de uma vizinhança maior, o lado direito da restrição local branching do atual nó é alterado para $k+\left\lceil\frac{k}{2}\right\rceil$. Esta etapa é denominada pelos autores de diversificação fraca. Se nenhuma solução de melhor qualidade for encontrada no limite de tempo, uma diversificação 


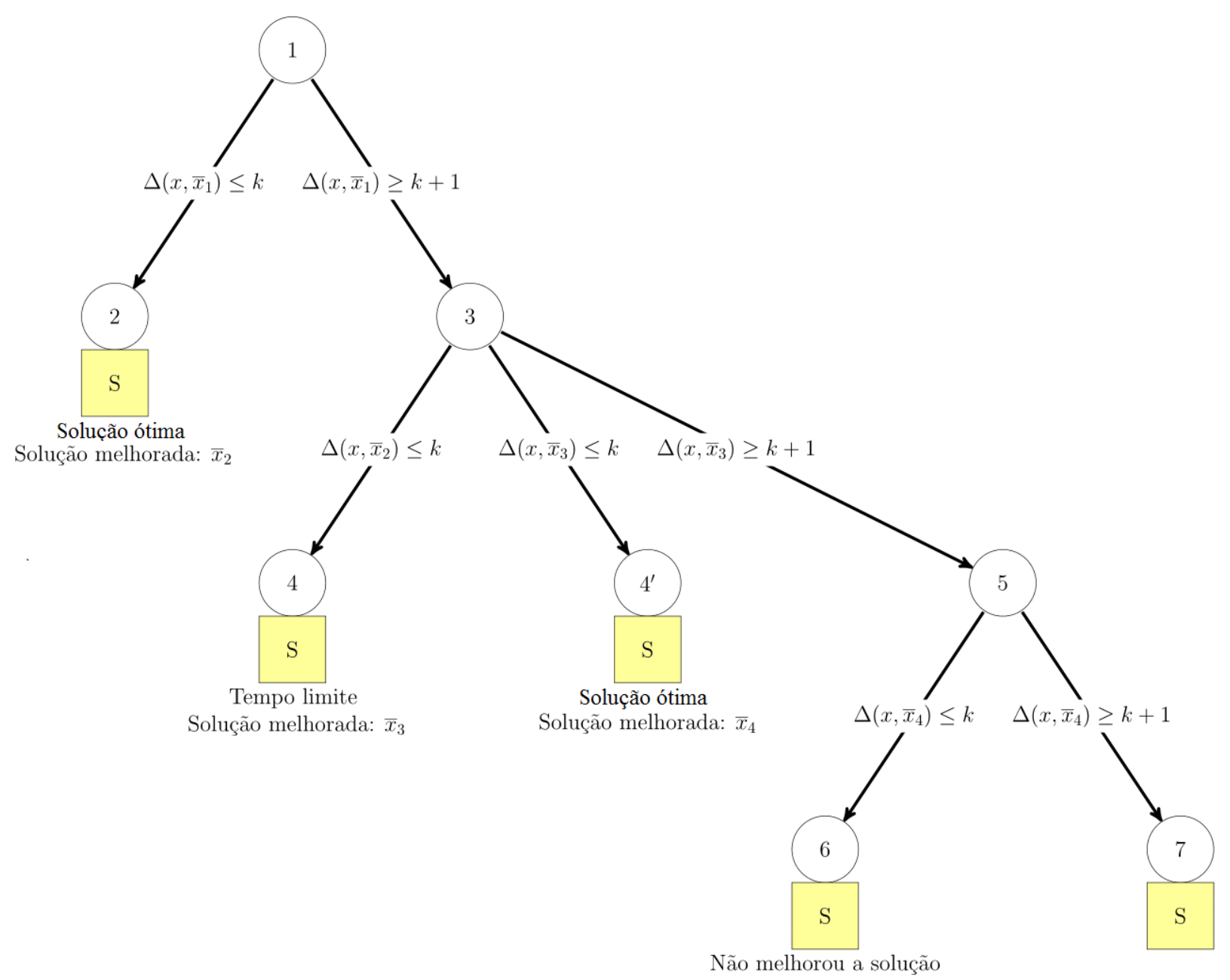

Figura 13 - Esquema do método LB com limite de tempo: solução incumbente atualizada

forte é aplicada alterando novamente o lado direito da restrição local branching para $k+$ $2\left\lceil\frac{k}{2}\right\rceil$ e a execução do subproblema é finalizada tão logo uma solução factível for encontrada, mesmo que essa seja pior que a solução incumbente. A etapa de diversificação forte é inspirada pelas metaheurísticas variable neighborhood search (MLADENOVIĆ; HANSEN, 1997), em que se procura uma solução pior que a atual, mas que esteja próxima da solução corrente. A solução obtida é utilizada como a solução de referência para as próximas vizinhanças.

As etapas de diversificação fraca e forte estão presentes, respectivamente, nos nós 4' e 4" da Figura 15. Como a solução ótima obtida pelo resolvedor, dentro do limite de tempo, no nó 4 não é melhor que a solução corrente, as restrições $\Delta\left(x, \bar{x}_{2}\right) \geq k+1$ e $\Delta\left(x, \bar{x}_{2}\right) \leq k+\lceil k / 2\rceil$ são acrescentadas ao subproblema corrente (nó 4'). Considerando, novamente, por hipótese que a solução ótima do subproblema associado ao nó 4' não é melhor que a atual, a restrição $\Delta\left(x, \bar{x}_{2}\right) \leq k+\lceil k / 2\rceil$ é substituída por $\Delta\left(x, \bar{x}_{2}\right) \geq k+\lceil k / 2\rceil+1$ e a diversificação forte é aplicada (nó 4"), obtendo uma nova solução de referência $\bar{x}_{3}$. Além disso, como as vizinhanças $\Delta\left(x, \bar{x}_{2}\right) \leq k$ e $\Delta\left(x, \bar{x}_{2}\right) \leq k+\lceil k / 2\rceil$ foram exploradas por completo, respectivamente, nos nós 4 e 4', as restrições $\Delta\left(x, \bar{x}_{2}\right) \geq k+1$ e $\Delta\left(x, \bar{x}_{2}\right) \geq k+\lceil k / 2\rceil+1$ são adicionadas no nó 5 .

O algoritmo 1 descreve todas as etapas do método LB. O algoritmo recebe como parâmetros de entrada o tamanho da vizinhança $(\mathrm{k})$, o limite de tempo de todo o procedimento (total_time_limit), o limite de tempo para explorar cada nó esquerdo (node_time_limit) 


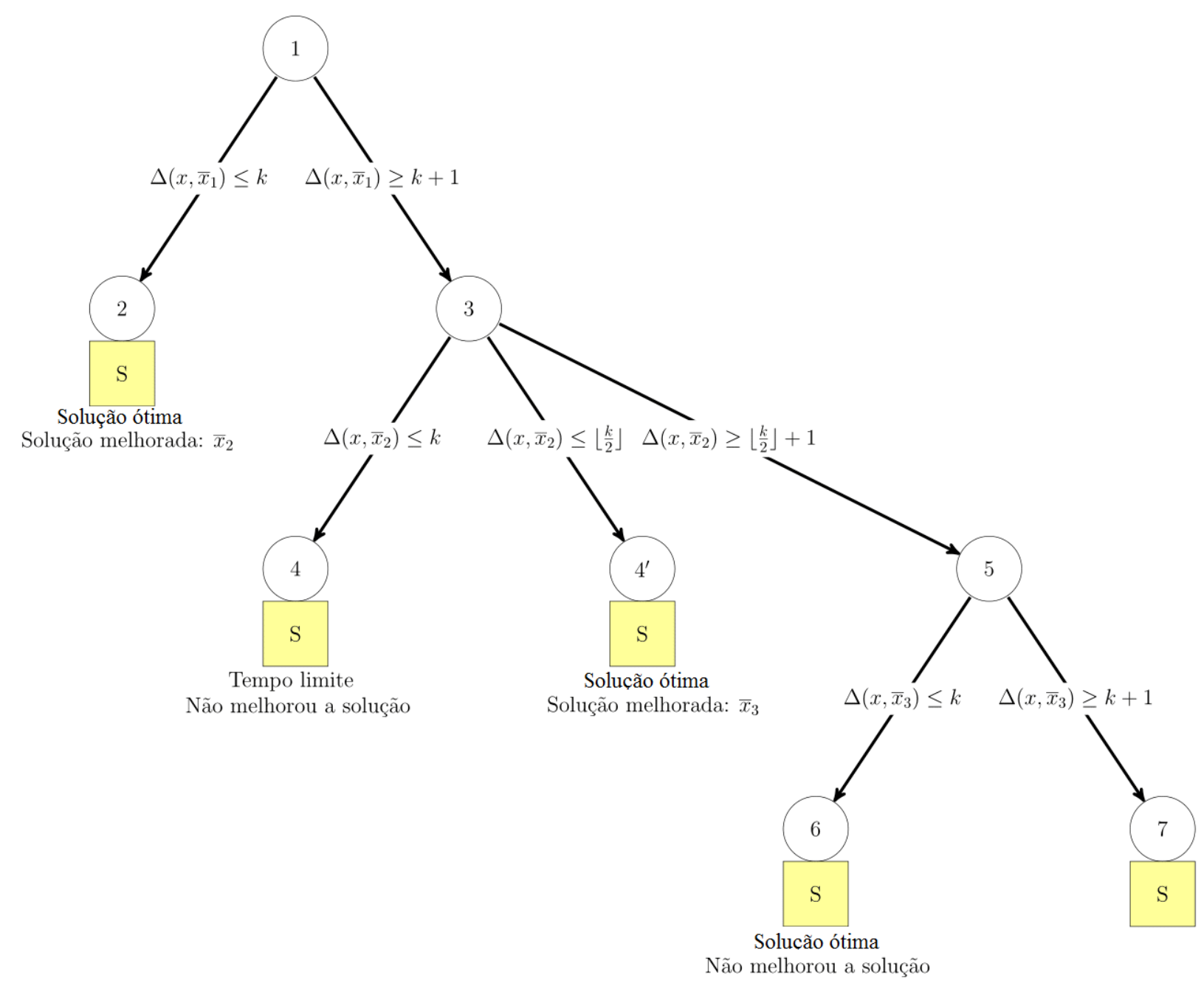

Figura 14 - Esquema local branching com limite de tempo: solução incumbente não atualizada

e o número máximo de diversificações fortes (diver_max). A saída de algoritmo é representada pela variável booleana opt e indica se a melhor solução encontrada $x^{*}$ é ótima ou não.

O método consiste na repetição do laço composto pelos comandos entre as linhas 4 e 39. A iteração é finalizada quando o limite de tempo total ou o número máximo de diversificações fortes é atingido. A cada iteração, um resolvedor é aplicado ao MILP corrente composto por 3 parâmetros: o limite de tempo para o processamento (TL), o limitante superior UB utilizado para interromper o processo de otimização assim que o melhor limitante inferior se torna maior ou igual a UB e a variável booleana "primeiro" que, caso tenha o valor verdadeiro, o resolvedor interrompe o processamento assim que encontrar a primeira solução factível. Além disso, a variável booleana "diversificação" indica o tipo de diversificação aplicada, sendo do tipo fraca caso a variável receba o valor falso e do tipo forte se receber o valor verdadeiro.

Quatro diferentes situações podem ocorrer após a resolução do MILP corrente:

a) solução_ótima_encontrada: a solução ótima do MILP corrente foi encontrada dentro do limite de tempo e o valor do limitante superior foi atualizado. Se o MILP representa algum nó esquerdo, a restrição local branching $\Delta(x, \bar{x}) \leq$ rhs é substituída pela restrição 


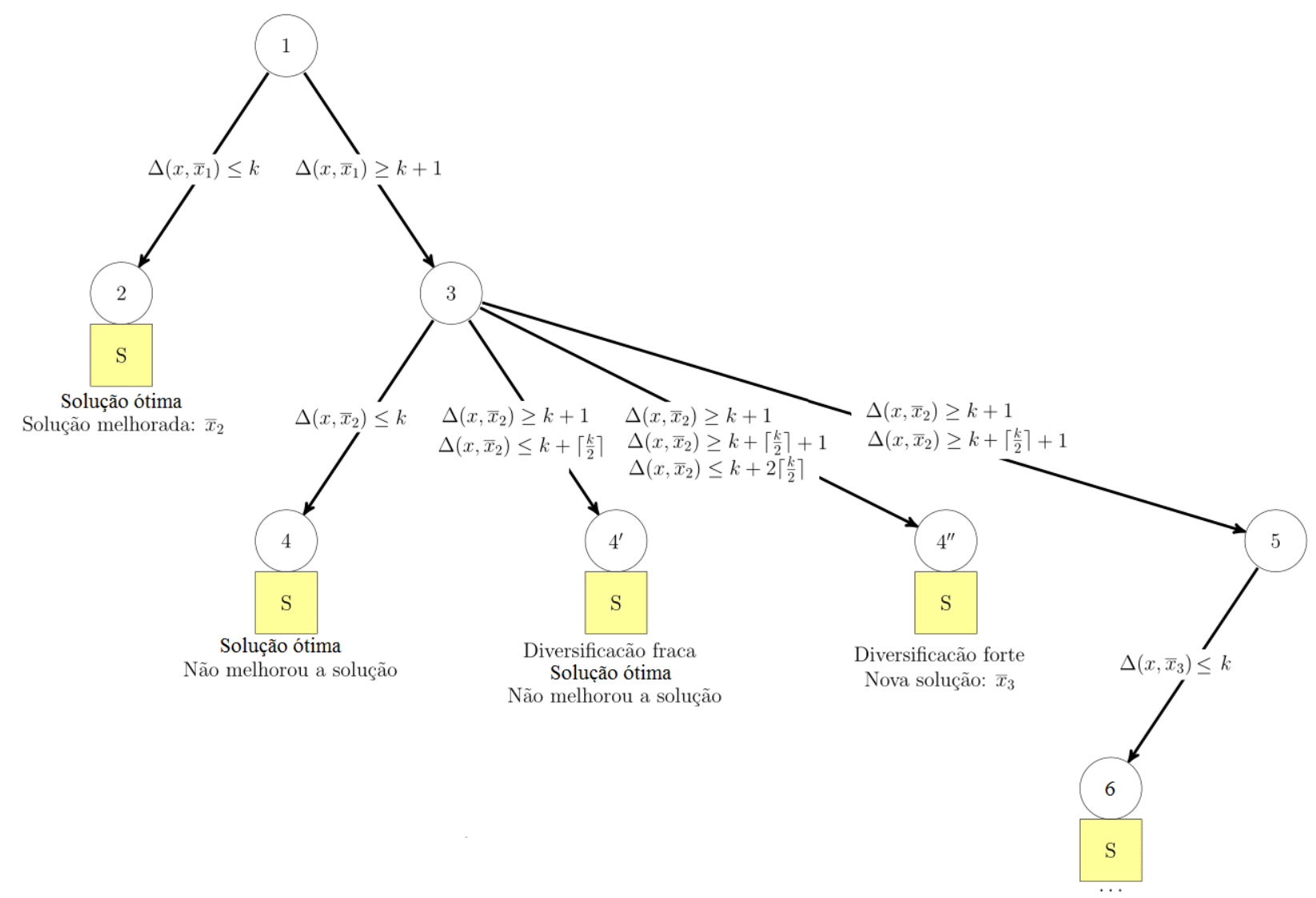

Figura 15 - Esquema local branching com estratégia de diversificação

$\Delta(x, \bar{x}) \geq \operatorname{rhs}+1$ (linha 12 ) e a solução referência $\bar{x}$ e o seu respectivo valor UB (e possivelmente a solução incumbente $x^{*}$ de valor bestUB) são atualizados (linhas 13 e 10). Se o MILP corresponde ao nó raiz, ou seja, ao problema (P), o algoritmo realiza um retorno imediato (linha 11).

b) provado_inviabilidade: a solução ótima do MILP corrente foi encontrada dentro do limite de tempo, mas o valor da função objetivo é maior que o valor do limitante superior UB. Se o MILP corresponde ao problema (P), o algoritmo faz um retorno imediato (linha 16). Caso contrário, a última restrição local branching é substituída por $\Delta(x, \bar{x}) \geq \mathrm{rhs}+1$ (linha 17$)$ e, conforme o valor da variável diversificação, é aplicado o mecanismo de diversificação fraca (linha 19) ou forte (linha 18).

c) solução_factivel_encontrada: uma solução factível $\tilde{x}$ do MILP corrente atualizou a solução corrente $\bar{x}$, mas não foi possível provar a sua otimalidade devido ao limite de tempo imposto ou pelo fato do resolvedor abortar o processamento ao encontrar a primeira solução factível no nó raiz. Como o respectivo MILP não foi explorado por completo, não podemos substituir a última restrição local branching por $\Delta(x, \bar{x}) \geq$ rhs +1 . Assim, a solução referência $\bar{x}$ e o seu respectivo valor UB (e possivelmente a solução incumbente $x^{*}$ de valor bestUB) são atualizados para a próxima iteração (linhas 25 e 26).

d) nenhuma_solução_factivel_encontrada: nenhuma solução factível com custo menor que UB foi encontrada dentro do limite de tempo. Devido à imposição do limite de 
tempo, não podemos garantir que tal solução não exista. Neste caso, dependendo do valor da variável diversificação, ou a última restrição local branching é excluída (linhas 34) ou substituída pela restrição $\Delta(x, \bar{x}) \geq 1$ (linhas 30 e 31 ). A restrição $\Delta(x, \bar{x}) \geq 1$ é inspirado na metaheurística busca tabu. Na busca tabu, para evitar que o método retorne a um ótimo local já visitado, algumas das soluções visitadas são armazenadas em um lista de soluções proibidas, ou lista tabu. Para evitar que a solução de referência apareça em etapas futuras, quando necessário, a restrição $\Delta(x, \bar{x}) \geq 1$ é adicionada ao MILP corrente. Se for aplicada a diversificação fraca, o tamanho da vizinhança de $\Delta(x, \bar{x})$ é reduzido (linha 35$)$, e no caso da diversificação forte, a vizinhança é aumentada e o limitante superior é inicializado com $\infty$ (linha 32).

Após o término do laço de repetição, a resolução do último nó à direita é limitada ao tempo de processamento restante.

A Figura 16 apresenta o comportamento do makespan da instância 02a do conjunto DPdata quando aplicado o método LB sem e com a etapa de diversificação. O tempo total de processamento foi de 3600 segundos. Nas abordagens LB, o tamanho da vizinhança (parâmetro k) é igual a 30, o limite de tempo para executar cada subproblema é 150 segundos, o número máximo de diversificação forte é igual a 3 e a solução inicial é representada pela segunda solução obtida pelo Gurobi. O valor do makespan no instante $t=0$ é infinito. A diversificação forte é aplicada nos instantes $t=1014, t=1655$ e $t=2946$. Nestes instantes, o limitante superior assume o valor infinito e o resolvedor busca novas soluções, mesmo com valores maiores do que a melhor solução encontrada. A melhor solução, de valor 2521, foi obtida pelo método LB sem diversificação. Ao comparar a evolução do limitante superior, observamos que o valor da solução incumbente do LB sem diversificação é melhor do que o valor da solução incumbente do LB com diversificação e do Gurobi em boa parte do tempo de processamento.

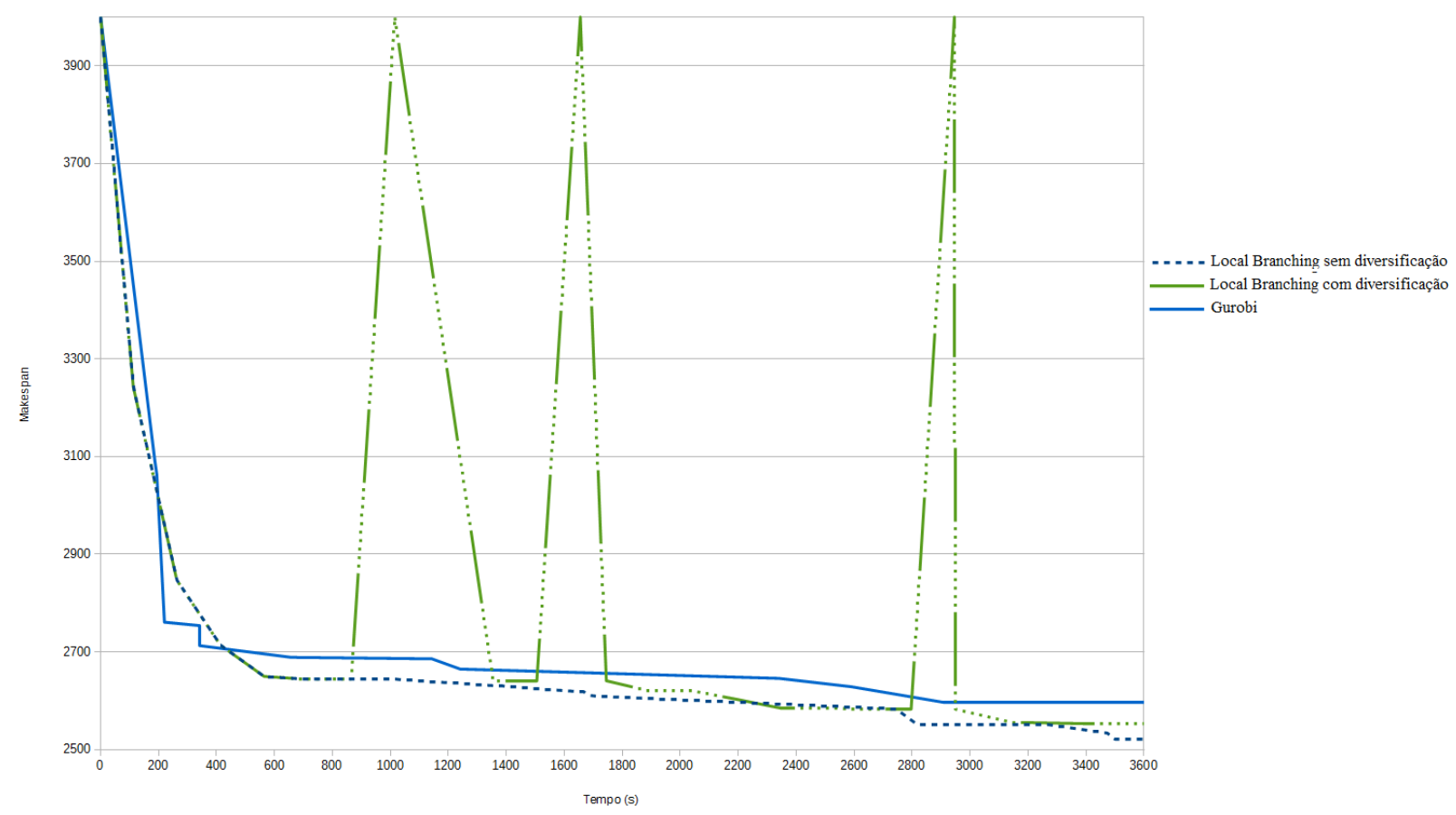

Figura 16 - Evolução do limitante superior da instância 02a do conjunto DPdata. 


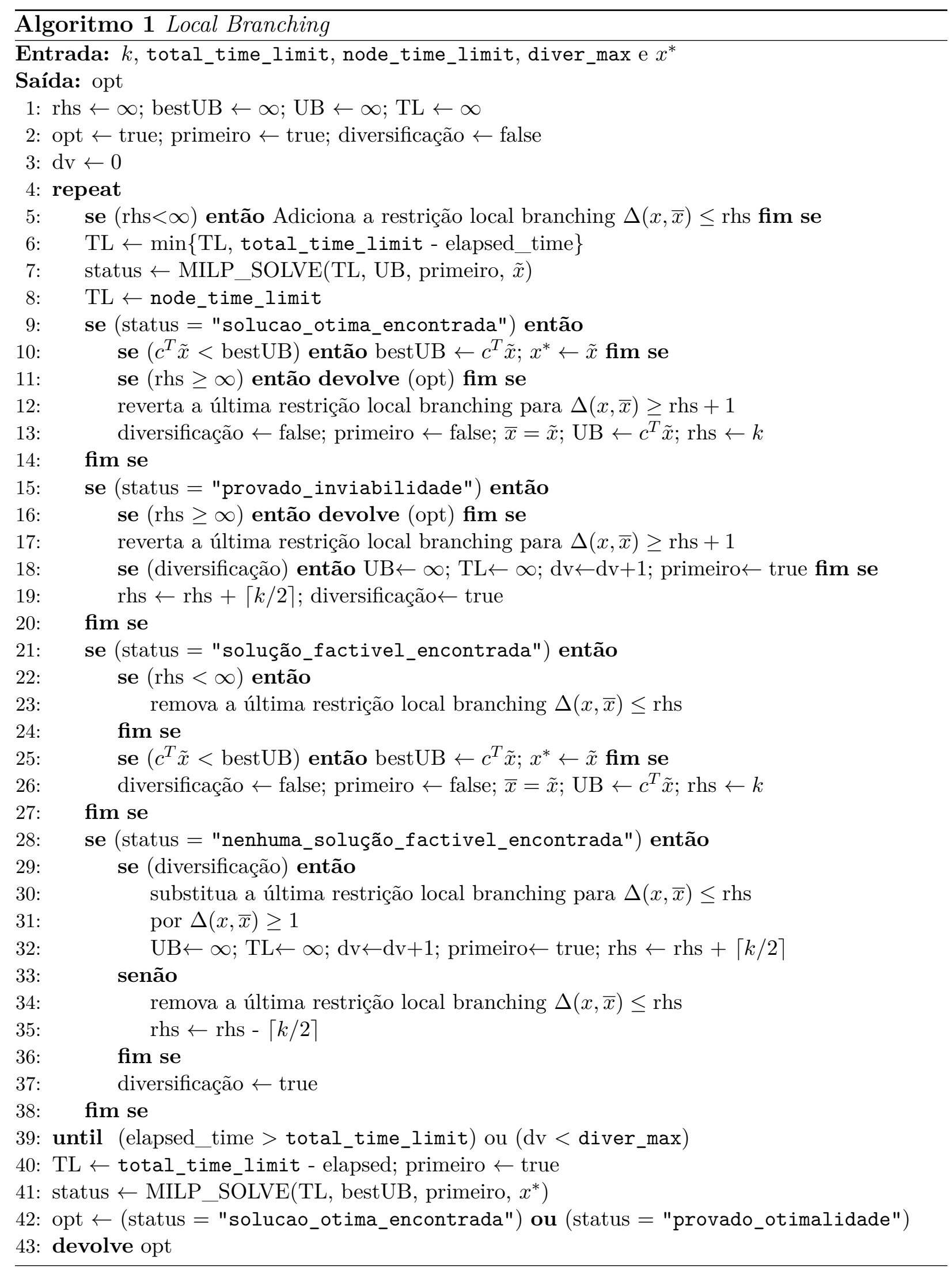


Devido aos bons resultados obtidos por Fischetti e Lodi (2003), em 2005, o método LB foi incorporado na versão 9.1 do CPLEX e é utilizado até o presente momento como um procedimento heurístico para encontrar novas soluções factíveis na árvore de busca. O CPLEX não permite alterar alguns dos parâmetros do LB, tais como o tamanho da vizinhança, o tempo de exploração do subMILP e habilitar ou desabilitar as diversificações fraca e forte. O resolvedor apenas permite alterar o parâmetro que controla o uso ou não do método.

Entretanto, o LB não faz parte do conjunto de heurísticas do Gurobi. Uma das heurísticas utilizadas pelo Gurobi para encontrar soluções factíveis é a relaxation induced neighborhood search (RINS). A matheurística RINS, proposta por Danna, Rothberg e Pape (2005), utiliza as informações da solução incumbente e da solução do problema relaxado para definir uma vizinhança. Essas soluções podem ter valores iguais para algumas de suas variáveis, e tais valores possam fazer parte de uma solução parcial para o problema. Para completar a solução, o método foca a atenção nas variáveis que diferem nas duas soluções. A RINS também é utilizada pelo CPLEX. Na sequência descrevemos a segunda matheuristics utilizada neste tese.

\subsection{Diversification, refining, and tight-refining}

Na conclusão do trabalho de Fischetti e Lodi (2003), os autores destacam a possibilidade de usar o método LB em um método parecido com o busca tabu ou variable neighborhood search. Com esse propósito, Fischetti, Polo e Scantamburlo (2004) apresentam uma heurística que combina os principais ingredientes dessas metaheurísticas dentro da resolução de um MILP e a aplicam, com sucesso, em um problema de localização de instalações de um projeto de rede de telecomunicações. O método desenvolvido é denominado de diversification, refining and tight-refining (DRT), sendo que cada termo que compõe o título representa uma etapa do método.

O método DRT é particularmente adequado a MILPs em que o conjunto de variáveis binárias pode ser dividido em dois subconjuntos ou níveis, de tal forma que, ao fixarmos os valores das variáveis do primeiro nível, o problema resultante seja mais simples (mas não trivial) de resolver. Este tipo de situação ocorre com JSF, pois uma vez definida em que máquina cada operação será processada (variável $x$ de MILP-1), o subproblema resultante equivale ao JS.

Para descrever o método, considere o problema (P) definido na seção 4.2 e tome como hipótese que o conjunto de variáveis binárias $\mathscr{B}$ é particionado em dois subconjuntos $\mathscr{B}=\left\{\mathscr{B}_{1}, \mathscr{B}_{2}\right\}$. Os subconjuntos $\mathscr{B}_{1}$ e $\mathscr{B}_{2}$ representam as variáveis do primeiro e segundo níveis, respectivamente.

Na fase de refinamento (refining) ou intensificação do DRT, dada uma solução factível $\bar{x}$, somente uma quantidade de variáveis do primeiro nível são deixadas livres e a vizinhança, composta por todas as possibilidades de combinação dessas variáveis, é explorada, na medida do possível, de forma eficiente. Esta etapa equivale a adicionar a restrição local branching

$$
\Delta_{1}(x, \bar{x})=\sum_{j \in \mathscr{B}_{1} ; \bar{x}_{j}=1}\left(1-x_{j}\right)+\sum_{j \in \mathscr{B}_{1} ; \bar{x}_{j}=0} x_{j} \leq k_{1}
$$

ao MILP corrente. Conforme os autores, o parâmetro $k_{1}$ é fixado em um valor baixo, por exemplo, $k_{1} \in\{0,1,2\}$. 
Se a solução ótima do MILP resultante não é encontrada dentro de um limite de tempo, a fase de ajuste-refinamento (tight-refining) é aplicada. Essa etapa consiste em explorar, além da vizinhança definida pela restrição (4.4), uma sequência de vizinhanças para a variável de segundo nível. Dessa forma, a restrição (4.4) é mantida ao problema e as variáveis do segundo nível são limitadas pela restrição

$$
\Delta_{2}(x, \bar{x})=\sum_{j \in \mathscr{B}_{2} ; \bar{x}_{j}=1}\left(1-x_{j}\right)+\sum_{j \in \mathscr{B}_{2} ; \bar{x}_{j}=0} x_{j}=k_{2} .
$$

O parâmetro $k_{2}$, que define o tamanho da vizinhança em (4.5), é incrementado conforme a sequência $k_{2}=0, k_{2}^{\text {passo }}, 2 k_{2}^{\text {passo }}, \cdots, k_{2}^{\max }$. Os valores $k_{2}^{\text {passo }}$ e $k_{2}^{\max }$ são atributos de entrada do algoritmo DRT. Dessa forma, o resolvedor explora, dentro de um limite de tempo, uma sequência de vizinhanças para a variável de segundo nível, sendo cada vizinhança contida na vizinhança definida por (4.4). A fase de ajuste-refinamento é finalizada quando $k_{2}$ atingir o valor máximo permitido ou quando o limite de tempo para esta fase é alcançado.

Finalizada a etapa de ajuste-refinamento, as restrições (4.4) e (4.5) são removidas do MILP corrente e iniciada a fase de diversificação (diversification). Assim como no método LB, o objetivo desta etapa é determinar uma nova solução, mas que esta seja suficientemente próxima da solução corrente, mesmo que seja de pior qualidade. No caso do método DRT, os valores das variáveis de primeiro nível da nova solução devem ser próximos da solução corrente. Dessa forma, a restrição de diversificação

$$
k_{1}^{\min } \leq \Delta_{1}(x, \bar{x}) \leq k_{1}^{\max }
$$

é adicionada ao modelo corrente. Logo, o número de variáveis binárias do primeiro nível da nova solução difere da solução anterior, no máximo $k_{1}^{\max } \mathrm{e}$, no mínimo, $k_{1}^{\min }$ valores. Um resolvedor é utilizado para obter uma solução factível para o MILP resultante e esta será a solução corrente $\bar{x}$ para a próxima iteração. Se nenhuma solução for encontrada no limite de tempo, os valores dos parâmetros $k_{1}^{\min }$ e $k_{1}^{\max }$ são modificados para estabelecer, de forma iterativa, vizinhanças de maior tamanho.

Assim como no método LB, a etapa de diversificação utiliza um procedimento inspirado na metaheurística busca tabu. A etapa de diversificação pode determinar por duas vezes a mesma configuração de valores para as variáveis de primeiro nível. Para evitar este problema, antes de iniciar a diversificação, a restrição tabu

$$
\Delta_{1}(x, \bar{x}) \geq 1
$$

é adicionada permanentemente ao MILP corrente.

O método DRT é descrito pelo algoritmo 2 e dividido em 3 sub-rotinas: diversificação (algoritmo 3), refinamento (algoritmo 4) e ajuste-refinamento (algoritmo 5). Os parâmetros do método são: tempo de processamento total (total_time_limit), tempo para obter a primeira solução (time_limit_first_solution), tempo de processamento relacionado a cada uma das fases (time_limit_diver, time_limit_ref, total_time_limit_ajus_ref e time_limit_ajus_ref) e a dimensão das vizinhanças $\Delta_{1}(x, \bar{x}) \mathrm{e} \Delta_{2}(\bar{x}, \bar{x})\left(k_{1}, k_{1}^{\text {ini }}, k_{1}^{\text {passo }}\right.$, $k_{2}^{\text {passo }}$ e $\left.k_{2}^{\max }\right)$. A função devolve a melhor solução encontrada dentro do limite de tempo. Se nenhuma solução for encontrada, o método retorna a uma solução vazia ( null). As 3 sub-rotinas têm como parâmetro de entrada a solução corrente $\bar{x}$.

A etapa inicial do algoritmo 2 consiste em determinar uma solução de referência $\tilde{x}$ (linha 2). Geralmente, a solução $\tilde{x}$ é obtida pela definição de um curto limite de tempo 
de processamento ou por fixar um número limite mínimo de soluções factíveis encontradas pelo resolvedor. A variável UB armazena o valor do limitante superior da solução corrente e bestUB o valor do melhor limitante superior. A variável booleana "primeiro", quando verdadeira, indica que o processo de otimização é finalizado pelo resolvedor assim que a primeira solução factível for encontrada. Uma vez que a solução corrente $\bar{x}$ esteja definida, as fases que definem o método DRT se alternam na estrutura de repetição entre as linhas 11-22. Dessa forma, o DRT se comporta como um método heurístico.

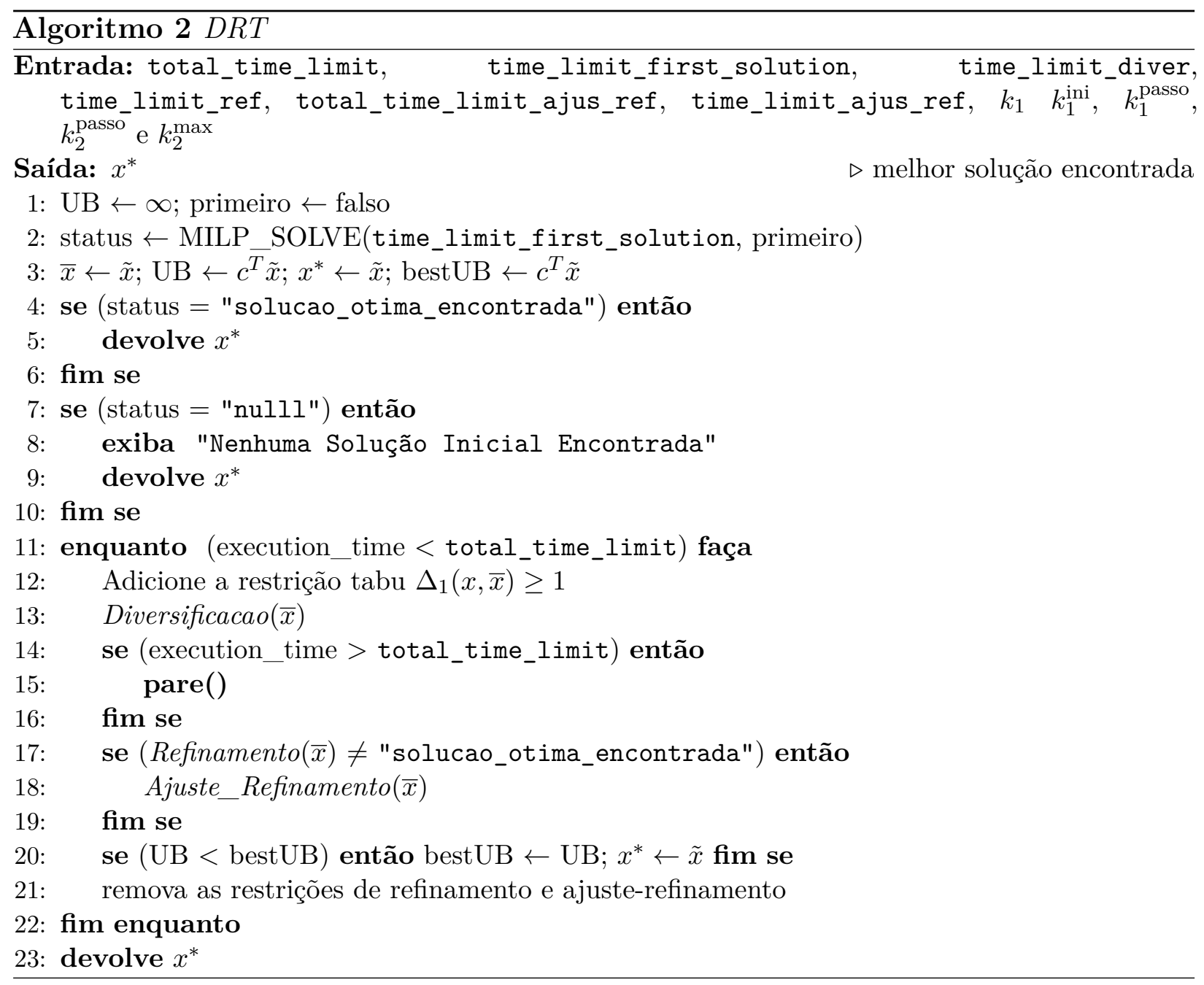

A sub-rotina de diversificação está descrita no algoritmo 3. Definido os valores mínimo e máximo $\left(k_{1}^{\min }\right.$ e $\left.k_{1}^{\max }\right)$ da vizinhança $\Delta_{1}(x, \bar{x})$ (linhas 1 e 3 ), a restrição de diversificação (4.6) é adicionada temporariamente ao problema corrente. Assim que a primeira solução factível para o problema for encontrada (linha 5), o processo de otimização é finalizado pelo resolvedor e a estrutura de repetição é concluída. Caso a solução não seja encontrada, as linhas 3 e 5 atualizam os valores $k_{1}^{\min }$ e $k_{1}^{\max }$ e o processo é repetido. Por fim, caso seja necessário, são atualizadas as soluções $\bar{x}$ e $x^{*}$ e seus respectivos custos.

O algoritmo 4 descreve a etapa de refinamento. Inicialmente, é adicionada a restrição de refinamento (4.4) ao problema corrente (linha 1) e a variável status armazena o resultado obtido pelo resolvedor. Se a solução ótima for encontrada, então a fase de ajuste-refinamento é descartada (linha 17 do algoritmo 2) e a restrição de refinamento é removida do problema (linhas 4-6). A sub-rotina é finalizada na linha 7, quando o valor da variável status é devolvido à rotina principal. 

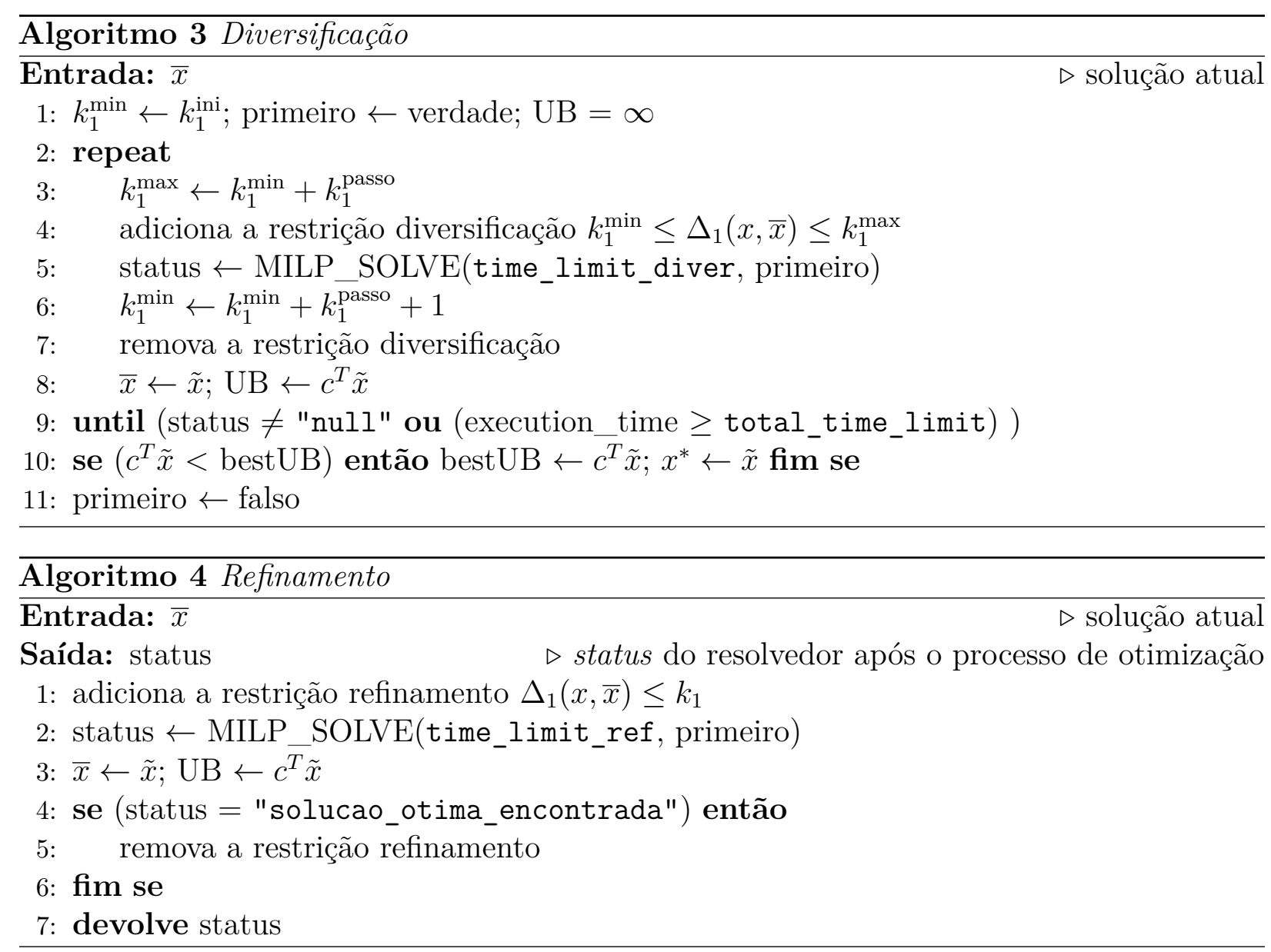

A sub-rotina de ajuste-refinamento é descrita no algoritmo 5. Enquanto o limite de tempo para esta etapa ou o tamanho da vizinhança $\Delta_{2}(x, \bar{x})$ não ultrapassar o seu valor máximo, o algoritmo adiciona a restrição $\Delta_{2}(x, \bar{x})=k_{2}$ ao problema corrente (linha 3). Ademais, o estado obtido pelo resolvedor é armazenado na variável status (linha 4) e o tamanho da vizinhança é atualizado (linha 10). Caso necessário, as soluções $\bar{x}$ e $x^{*}$ também são atualizadas (linhas 5-9).

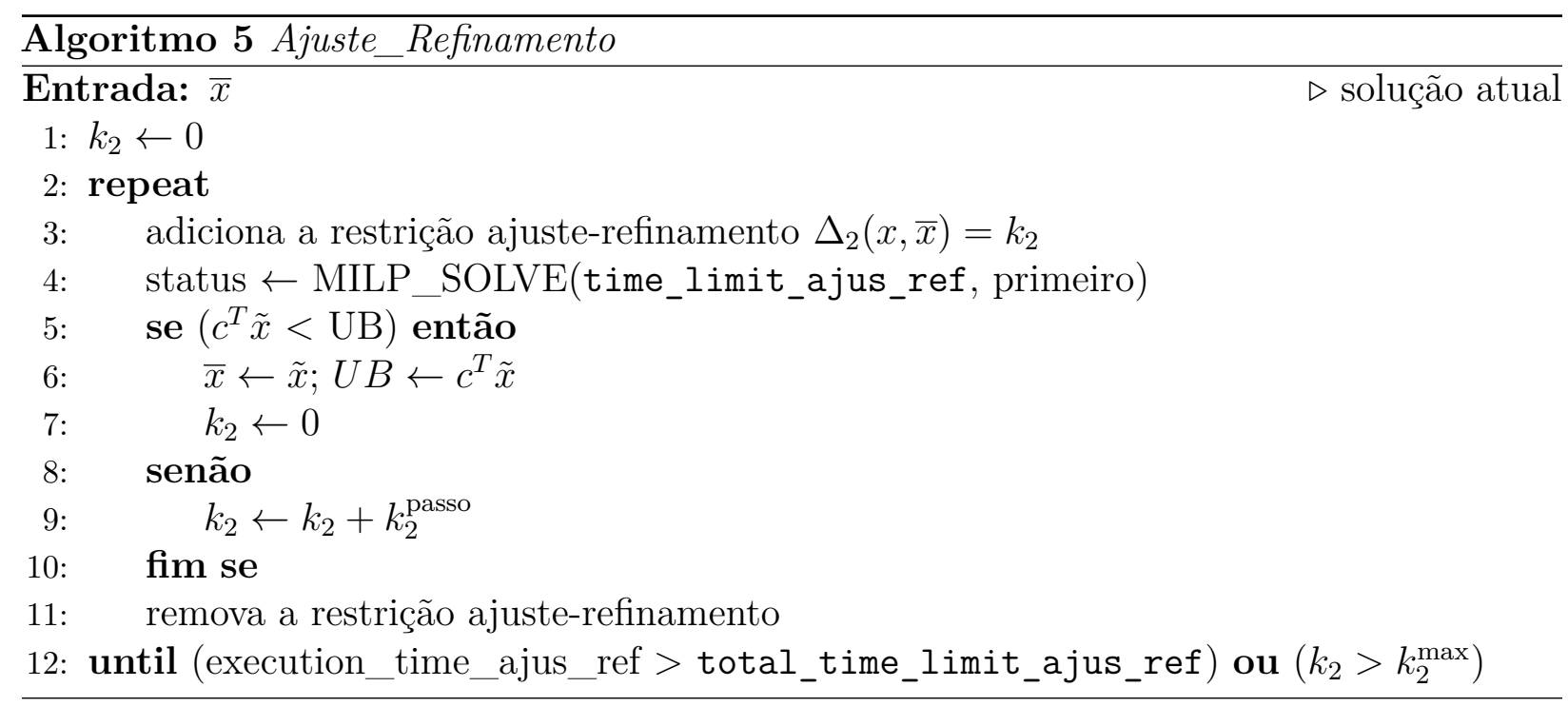

A função MILP_SOLVE() presente nos algoritmos 2, 3, 4 e 5 representa a chamada do resolvedor e tem como parâmetros o limite de tempo para a fase em que é aplicada e o 
limite do número de soluções. Outros parâmetros podem ser adicionados, tais como, ênfase na estratégia de obter a solução factível e valor de corte para o limite superior. A função retorna à melhor solução encontrada conforme a configuração dos parâmetros. Se nenhuma solução for encontrada, o retorno é uma solução vazia.

A Figura 17 apresenta a evolução do limitante superior da instância MK09 do conjunto BRdata para o Gurobi e o método DRT. O tempo de execução é de 3600 segundos. No instante $t=0$, o makespan assume o valor infinito. A solução inicial do DRT, de valor $426(t=53$ segundos), é obtida após o Gurobi encontrar a segunda solução factível. A etapa de diversificação é iniciada em três momentos: $t=66, t=1366$ e $t=2700$ segundos. Nestes instantes, a partir da solução de referência, o método inicia a fase de refinamento. $\mathrm{O}$ tempo para cada etapa de refinamento é de 450 segundos. Como as etapas de refinamento foram finalizadas sem encontrar o valor ótimo, o DRT inicia, na sequência, a etapa de ajuste-refinamento. A melhor solução, de valor 352, é obtida pelo método DRT no instante $t=3300$ segundos, durante a fase de ajuste-refinamento.

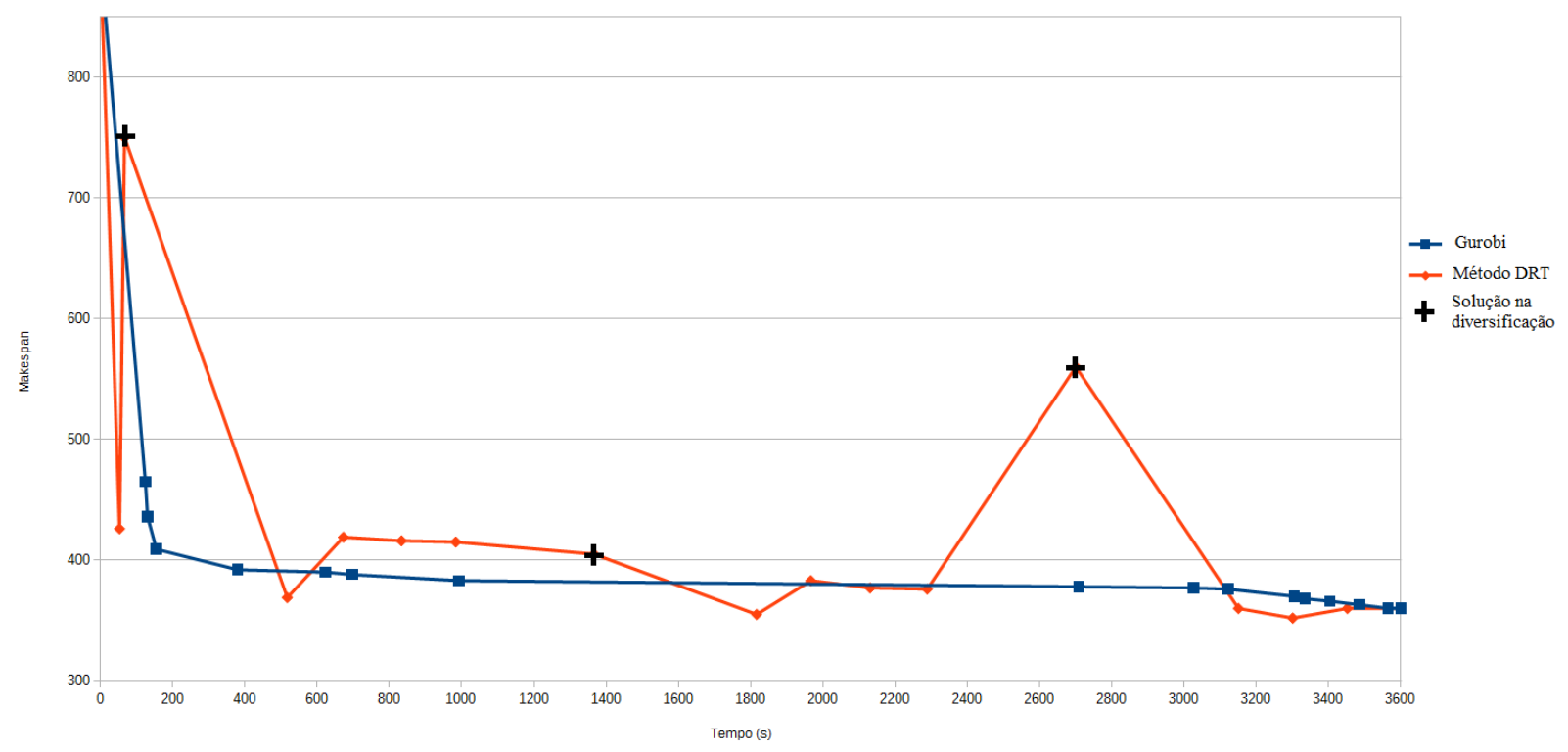

Figura 17 - Evolução do limitante superior da instância MK09 do conjunto BRdata

\subsection{Experimentos computacionais}

Nesta seção, apresentamos os experimentos computacionais dos métodos LB e DRT. Os experimentos foram realizados em um computador Intel i7 com processador $2.93 \mathrm{GHz}(6$ GB RAM). As implementações foram realizadas na linguagem Java e o pacote de otimização Gurobi, versão 6.5, foi utilizado na resolução dos problemas e subproblemas de otimização.

Os dois métodos foram avaliados para as instâncias da seção 3.5, com exceção das instâncias sfjs01-sfjs1 do conjunto Fdata. Tais instâncias são de menor dimensão e não foram levadas em consideração pois as soluções são obtidas em MILP-1 em menos de 1 segundo. Utilizamos somente os problemas mfs01-mfs10 do conjunto Fdata na avaliação dos métodos. Dessa forma, foram utilizadas 59 instâncias teste. Para a execução de cada instância, nós impomos um limite de tempo de 3600 segundos. 


\subsubsection{Experimentos computacionais para o método local branching}

Com o objetivo de verificar o comportamento do método LB na resolução do JSF, foram realizadas duas implementações utilizando o modelo MILP-1 do JSF: LB sem a etapa de diversificação, o qual será denominado de LB_SD, e LB com a etapa de diversificação, denominado de LB_CD. Nas duas abordagens, foram utilizadas a estratégia de fixar um limite de tempo para a resolução dos subMILPs. Dividimos a abordagem LB em dois algoritmos pois objetivamos verificar a influência do mecanismo de diversificação na qualidade das soluções. Os resultados de LB_SD e LB_CD são comparados com o Gurobi, usando o modelo MILP-1, em sua configuração padrão.

O método LB utiliza o parâmetro $k$ para estabelecer o tamanho da vizinhança e o parâmetro $t_{\text {left }}$ para definir o limite de tempo para a execução de cada subMILP esquerdo. No trabalho de Fischetti e Lodi (2003), os autores não descrevem o procedimento adotado para estabelecer o valor de cada parâmetro. Dessa forma, a metodologia para a escolha do melhor parâmetro em cada método e em cada conjunto é a mesma utilizada em Danna, Rothberg e Pape (2005) para calibrar os parâmetros de LB e da matheuristic RINS. Em sua dissertação de mestrado, Santos (2006) também utiliza a mesma abordagem para calibrar os parâmetros e comparar a evolução do limitante superior da função objetivo (problema de minimização) em diferentes variações do LB e RINS.

Para calibrar os parâmetros $k$ e $t_{l e f t}$, foram selecionadas, de forma aleatória e proporcional ao tamanho do conjunto, 18 instâncias dos conjuntos BRdata, DPdata, Fdata e BCdata: mf js08, mfjs10, MK01, MK04, MK08, mt10x, mt10xy, setb4x, setb4xxx, seti5c12, seti5x, seti5xy, 02a, 04a, 07a, 11a, 14a e 16a. Definimos 3 valores para cada parâmetro: $k \in\{10,20,30\}$ e $t_{\text {left }} \in\{150,200,250\}$. Na primeira etapa de calibração no conjunto teste, o parâmetro $k$ variou e o tempo de execução do subMILP ficou fixo em 200 segundos. Para a escolha do melhor valor do parâmetro $k$, o intervalo de tempo é dividido em 36 subintervalos iguais. No término de cada subintervalo, é calculada a razão entre o melhor valor do limitante superior obtido até o presente momento e o melhor valor obtido no final da execução dos 3 testes para a instância, sendo cada teste representado por um valor fixo de $k$. Para cada subintervalo e para cada teste, calculamos a média geométrica destas razões para todas as instâncias. O melhor valor para o parâmetro é aquele em que a média geométrica do teste tiver o maior número de valores próximos de 1, dentre os 36 subintervalos.

Para exemplificar, a tabela 27 apresenta, para cada experimento $(k=10,20,30)$, o melhor valor obtido pelo makespan pela abordagem LB_SD para as 18 instâncias teste no instante $t=100$ segundos. Fixamos $t_{\text {left }}$ no valor 200 . A coluna $C_{\text {Max }}^{*}$ denota o melhor valor obtido pelo limitante superior após o término dos três experimentos e $C_{\text {Max }}$ o valor do makespan em $t=100$ segundos. O experimento $k=10$ é o que tem o valor da média geométrica das razões mais próximo de 1 . O procedimento é repetido para os demais 35 subintervalos.

As figuras 18a e 18b apresentam a curva da média geométrica de cada teste para os métodos LB_SD e LB_CD, respectivamente. Em ambos os métodos, o melhor valor para o parâmetro $k$ foi obtido quando o tamanho da vizinhança é fixado no valor 30 .

A próxima etapa do processo de calibração baseou-se na definição do valor do parâ-

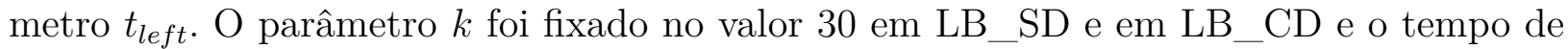
execução do subMILP foi variado. A definição do melhor valor para $t_{l e f t}$ é análogo ao processo anterior. As figuras 19a e 19b apresentam a média geométrica de cada teste, considerando $t_{\text {left }} \in\{150,200,250\}$. Em ambos os métodos, o melhor valor para $t_{\text {left }}$ é obtido quando este 
Tabela 27 - Exemplo da etapa de calibração no instante $t=100$ segundos.

\begin{tabular}{|c|c|c|c|c|c|c|c|}
\hline \multirow[b]{2}{*}{ Instância } & \multirow[b]{2}{*}{$C_{\text {Max }}^{*}$} & \multicolumn{2}{|c|}{$k=10$} & \multicolumn{2}{|c|}{$k=20$} & \multicolumn{2}{|c|}{$k=30$} \\
\hline & & $C_{\text {Max }}$ & Razão & $C_{\text {Max }}$ & Razão & $C_{\text {Max }}$ & Raz \\
\hline mfjs 08 & 884 & 884 & 1 & 884 & 1 & 1247 & 1,41 \\
\hline mfjs10 & 1227 & 1254 & 1,02 & 2075 & 1,69 & 1827 & 1,48 \\
\hline MK01 & 40 & 40 & 1 & 42 & 1,05 & 50 & 1,12 \\
\hline M & 60 & 67 & 1 , & 79 & 1, & 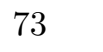 & 1,22 \\
\hline MK & 528 & 569 & 1,0 & 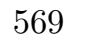 & 1, & 9 & 1,07 \\
\hline $\operatorname{mt} 10$ & 918 & 918 & 1 & 1084 & 1,18 & 1032 & 1,12 \\
\hline t10xy & 905 & 905 & 1 & 1209 & 1,33 & 1142 & 1,26 \\
\hline tb4x & 925 & 937 & 1,012 & 1203 & 1,30 & 1156 & 1,24 \\
\hline setb & 925 & 925 & 1 & 10923 & 1, & 1069 & 1,1 \\
\hline seti5 & 1175 & 1246 & 1, & 1426 & 1,21 & 1391 & 1,18 \\
\hline seti5x & 1202 & 1259 & 1,04 & 1353 & 1,12 & 1331 & 1,10 \\
\hline seti5x & 1136 & 1216 & 1,07 & 1367 & 1,20 & 1317 & 1,15 \\
\hline $02 \mathrm{a}$ & 2451 & 2913 & & 2802 & & 2752 & 1,12 \\
\hline 8 & 2697 & 3500 & 1,29 & 3274 & 1,21 & 3157 & 1,17 \\
\hline $07 \mathrm{a}$ & 2641 & 3025 & 1,14 & 2948 & 1,11 & 2917 & 1,10 \\
\hline $11 \mathrm{a}$ & 2390 & 2971 & 1,24 & 2810 & 1,17 & 2661 & 1,11 \\
\hline $14 \mathrm{a}$ & 2628 & 3303 & 1,25 & 2932 & 1,11 & 2951 & 1,12 \\
\hline $16 \mathrm{a}$ & 2643 & 2950 & 1,11 & 2923 & 1,10 & 2873 & 1,08 \\
\hline M. Geom. & & & 1,08 & & 1,18 & & 1,17 \\
\hline
\end{tabular}

assume o valor 150 .

No método LB, quando é atribuído o valor Verdade para a variável booleana "primeiro", o resolvedor finaliza o processamento do nó assim que encontrar a primeira solução factível. No Gurobi, este procedimento é obtido configurando o parâmetro SolutionLimit. Mas nem sempre a primeira solução é de boa qualidade e, dessa forma, quando necessário, configuramos em LB_SD e LB_CD para que o Gurobi interrompa o processo de otimização ao encontrar a segunda solução factível.

Conforme Fischetti e Lodi (2003), durante a execução do laço de repetição entre as linhas 4-39 do algoritmo 1, espera-se do método LB encontrar rapidamente cada vez melhores soluções até chegar a um ponto em que as restrições não possam mais ser aplicadas. Assim, para auxiliar este processo, durante a execução do laço, configuramos o parâmetro MIPFocus para o valor 1. O parâmetro MIPFocus permite modificar a estratégia de obter uma solução dependendo de seu objetivo. Quando parametrizado como MIPFocus $=1$, entende-se que o usuário está interessado em encontrar uma solução factível mais rapidamente. Na configuração padrão, o Gurobi estabelece um equilíbrio entre encontrar uma solução factível e provar a sua otimalidade. Antes de processar por completo o último nó à direita (linha 41 do algoritmo 1), o parâmetro MIPFocus é configurado em seu valor padrão.

As variáveis binárias $x$ e $y$ de MILP-1 foram utilizadas para descrever o conjunto suporte binário $\bar{S}$ (4.1) e as restrições local branching (4.2).

Os resultados obtidos para as abordagens MILP-1-Gurobi, LB_SD e LB_CD estão 


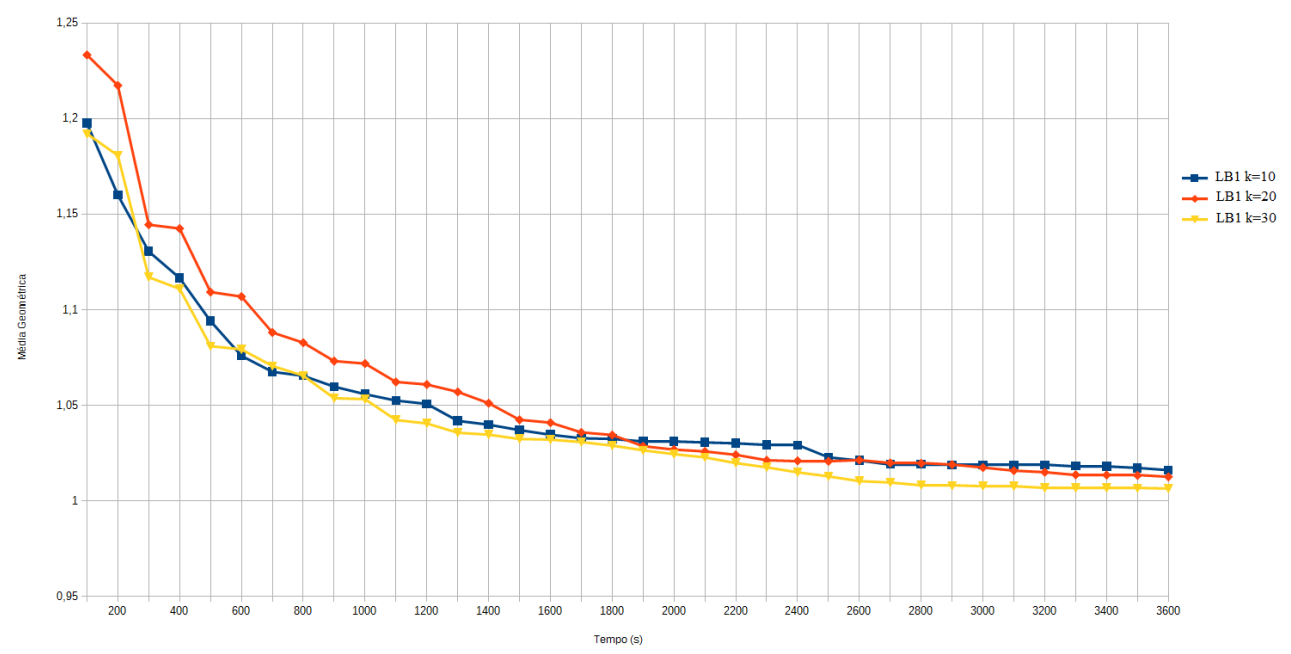

(a) Variação do parâmetro $k$ em LB_SD

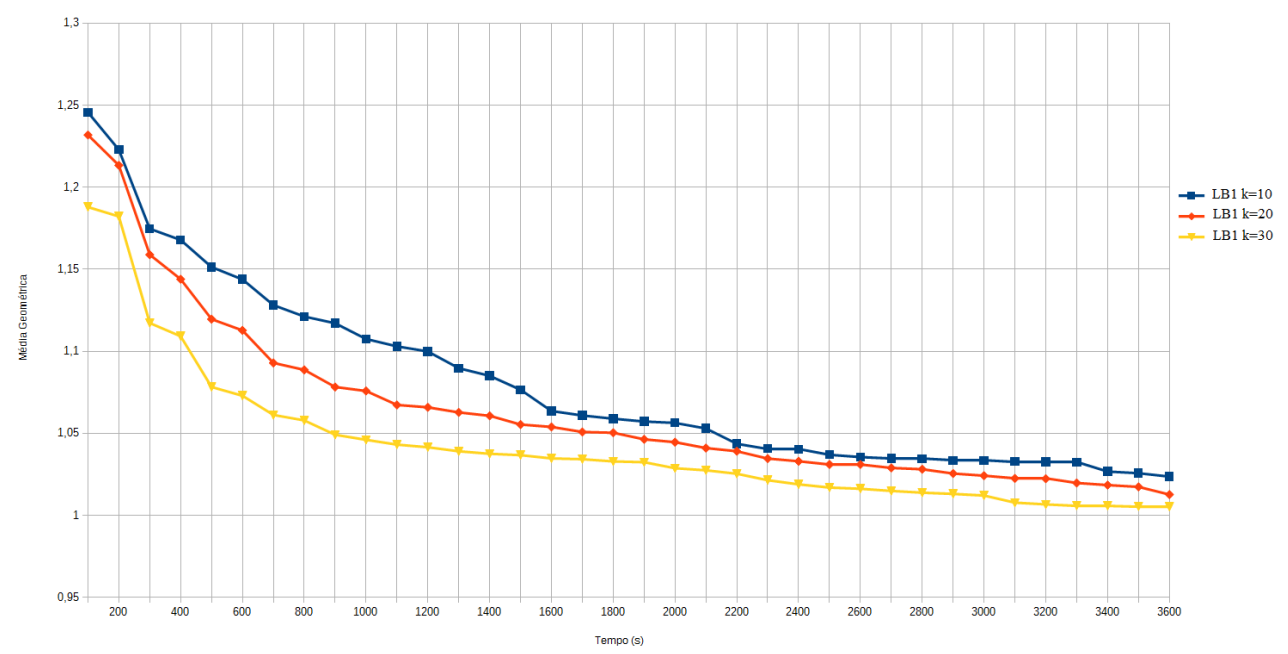

(b) Variação do parâmetro $k$ em LB_CD

Figura 18 - Etapa de calibração do parâmetro $k$.

descritos nas tabelas 28, 29, 30 e 31. As colunas de cada tabela apresentam o nome da instância teste, o valor do makespan e do tempo de processamento (em segundos) para cada uma das abordagens. Os valores do makespan e do tempo de processamento de cada instância em MILP-1-Gurobi desta seção diferem dos valores apresentados na seção 3.5, pois a configuração do computador e a versão do Gurobi são diferentes.

Para cada instância, destacamos em negrito o melhor valor do makespan quando este for obtido, no máximo, por duas abordagens. Se nenhum valor for destacado, então as três abordagens tiveram o mesmo valor do makespan para a instância de teste.

De acordo com as tabelas 28, 29, 30 e 31, a abordagem LB_SD obteve o melhor valor para o makespan em 38 instâncias, sendo que, em 31 dessas instâncias, o mesmo valor do makespan também foi obtido por outra abordagem. A abordagem LB_CD obteve o melhor valor do makespan em 24 instâncias. Desse total, em 7 instâncias, a melhor solução foi obtida somente por LB_CD. De forma geral, as abordagens que envolvem a técnica LB superaram a solução de MILP-1-Gurobi em 14 instâncias, o que corresponde a 23,73\% dos problemas analisados. Mas nenhuma destas soluções representa solução ótima para o problema. Além disso, em 33 instâncias, o valor da solução obtida por LB_SD ou LB_CD coincidiu com o 


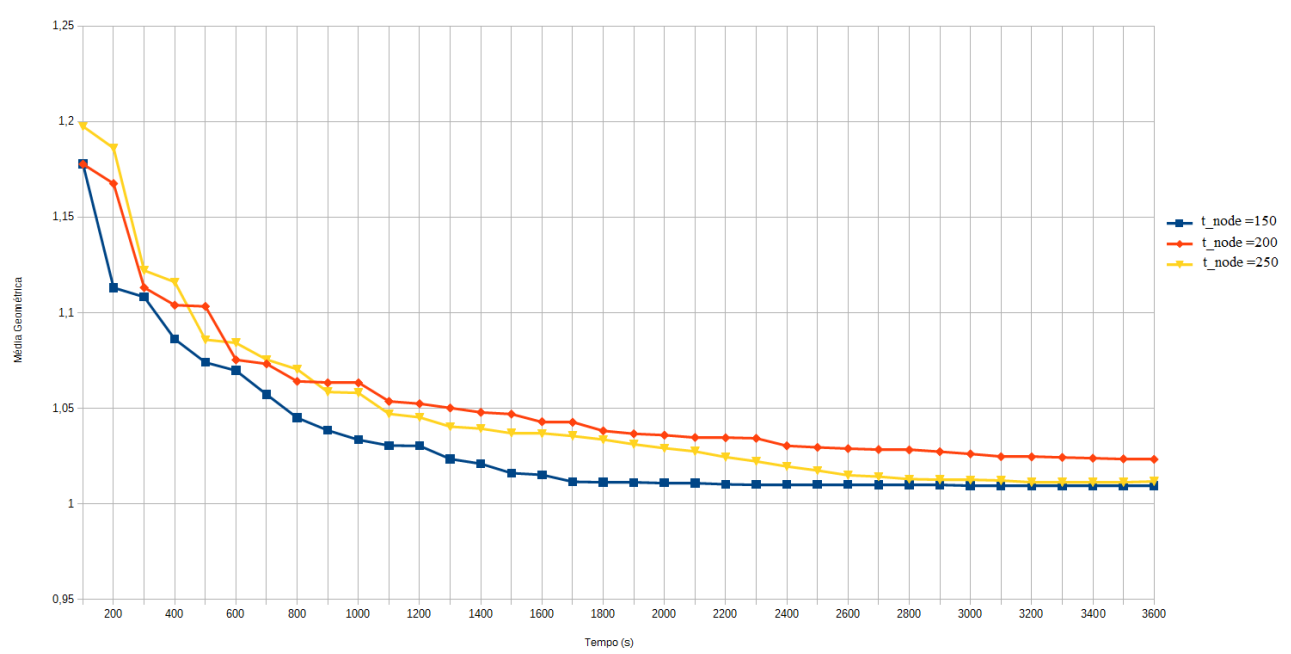

(a) Variação do parâmetro $t_{l e f t}$ em LB_SD

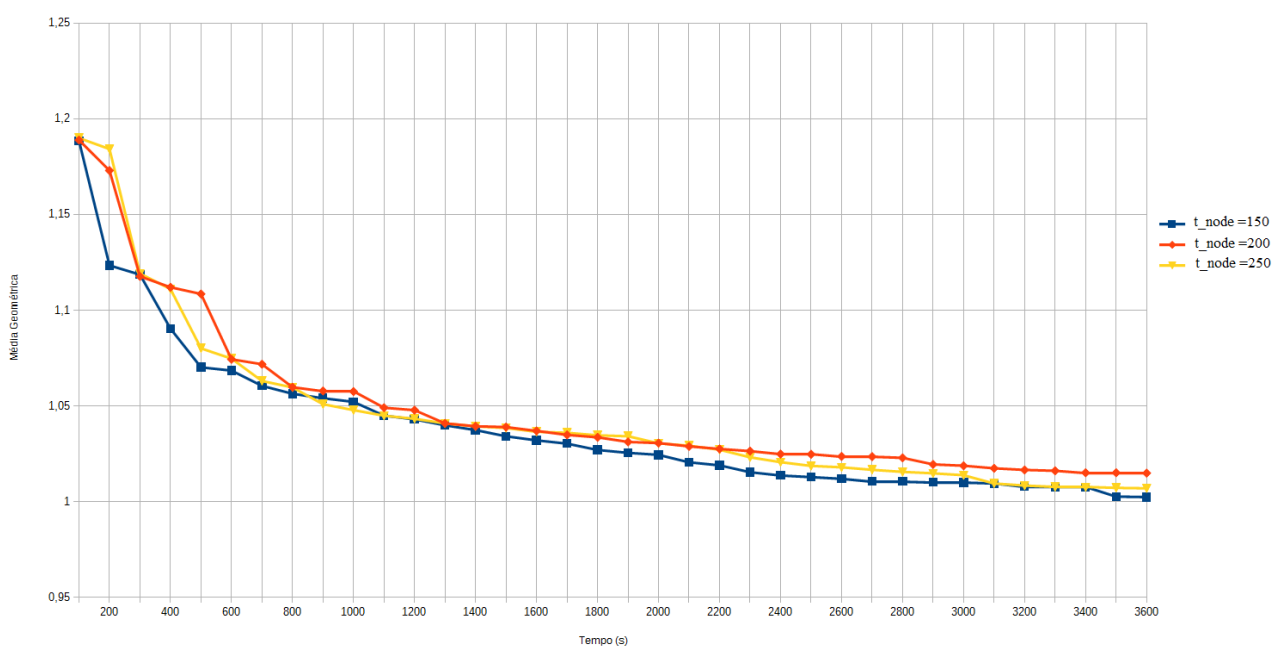

(b) Variação do parâmetro $t_{l e f t}$ em LB_CD

Figura 19 - Etapa de calibração do parâmetro $t_{l e f t}$.

valor de MILP-1. Desta forma, observamos que estratégia de implementar duas abordagens do método LB foi eficiente, pois as abordagens com e sem o mecanismo de diversificação contribuíram para a melhoria da solução.

Considerando a quantidade de melhores soluções obtidas pelas duas abordagens LB, o melhor desempenho foi no conjunto DPdata (tabela 31). Neste conjunto, as soluções obtidas por LB_SD e LB_CD superaram MILP-1-Gurobi em 10 instâncias.

Em todas as instâncias em que MILP-1 obteve a solução ótima, o mesmo valor para o makespan foi obtido em LB_SD ou LB_CD. Para estes problemas, exceto para a instância setb4x, o tempo total de processamento nas abordagens LB_SD e LB_CD foi superior. Isto ocorre pelo fato do Gurobi explorar diversos subMILPs durante todo o período de otimização. As soluções ótimas obtidas por MILP-1-Gurobi são aquelas em que o tempo de processamento é inferior a 3600 segundos, além do problema setb4x. Embora o makespan ótimo da instância setb4x seja igual a 925, o resolvedor não conseguiu provar a sua otimalidade.

Observamos que, no conjunto DPdata, a solução em LB_SD foi obtida explorando os subMILPs esquerdos para 9 das 17 instâncias, enquanto para LB_CD, a exploração dos 
Tabela 28 - Resultados computacionais de MILP-1-Gurobi, LB_SD e LB_CD para o conjunto Fdata.

\begin{tabular}{|c|c|c|c|c|c|c|}
\hline \multirow[t]{2}{*}{ Problema } & \multicolumn{2}{|c|}{ MILP-1-Gurobi } & \multicolumn{2}{|c|}{ LB_SD } & \multicolumn{2}{|c|}{$\mathrm{LB} \_\mathrm{CD}$} \\
\hline & $C_{\text {Max }}$ & Tempo(s) & $C_{M a x}$ & Tempo(s) & $C_{\operatorname{Max}}$ & Tempo(s) \\
\hline mfjs01 & 468 & 0,19 & 468 & 0,72 & 468 & 5,41 \\
\hline mfjs02 & 446 & 0,14 & 446 & 0,93 & 446 & 4,88 \\
\hline mfjs03 & 466 & 0,25 & 466 & 1,47 & 466 & 5,74 \\
\hline mfjs04 & 554 & 0,69 & 554 & 11,01 & 554 & 62,06 \\
\hline mfjs05 & 514 & 0,53 & 514 & 7,47 & 514 & 18,35 \\
\hline mfjs06 & 634 & 1,76 & 634 & 40,76 & 634 & 117,33 \\
\hline mfjs07 & 879 & 14,16 & 879 & 288,37 & 879 & 830,01 \\
\hline mfjs08 & 884 & 342,39 & 884 & 2346,83 & 884 & 3600 \\
\hline mfjs09 & 1073 & 3600 & 1073 & 3600 & 1073 & 3600 \\
\hline mfjs10 & 1214 & 3600 & 1232 & 3600 & 1216 & 3600 \\
\hline
\end{tabular}

Tabela 29 - Resultados computacionais de MILP-1-Gurobi, LB_SD e LB_CD para o conjunto BRdata.

\begin{tabular}{|c|c|c|c|c|c|c|}
\hline \multirow[t]{2}{*}{ Problema } & \multicolumn{2}{|c|}{ MILP-1-Gurobi } & \multicolumn{2}{|c|}{ LB_SD } & \multicolumn{2}{|c|}{$\mathrm{LB} \_\mathrm{CD}$} \\
\hline & $C_{\text {Max }}$ & Tempo(s) & $C_{M a x}$ & Tempo(s) & $C_{\text {Max }}$ & Tempo(s) \\
\hline Mk01 & 40 & 182,14 & 40 & 585,41 & 40 & 3600 \\
\hline $\mathrm{Mk} 02$ & 26 & 3600 & 27 & 3600 & 26 & 3600 \\
\hline Mk03 & 204 & 3600 & 204 & 3600 & 204 & 3600 \\
\hline Mk04 & 60 & 3600 & 60 & 3600 & 65 & 3600 \\
\hline Mk05 & 177 & 3600 & 177 & 3600 & 179 & 3600 \\
\hline Mk06 & 70 & 3600 & 70 & 3600 & 69 & 3600 \\
\hline $\mathrm{Mk} 07$ & 170 & 3600 & 153 & 3600 & 171 & 3600 \\
\hline Mk08 & 524 & 3600 & 524 & 3600 & 524 & 3600 \\
\hline Mk09 & 360 & 3600 & 349 & 3600 & 361 & 3600 \\
\hline Mk10 & 250 & 3600 & 315 & 3600 & 320 & 3600 \\
\hline
\end{tabular}

subMILPs esquerdos encontrou a solução para 12 das 16 instâncias. Especificamente para este conjunto, em que a dimensão das instâncias é superior aos demais conjuntos, isto evidencia uma das características da técnica LB, que consiste em encontrar boas e rápidas soluções ao explorar os subMILPs referentes às ramificações à esquerda. Para a maioria das instâncias dos conjuntos BRdata e BCdata, ambas as abordagens obtiveram as soluções otimizando o último nó à direita. Nestas instâncias, no problema associado ao último nó à direita, além de incorporar a última restrição local branching, apresentam as restrições oriundas dos subMILPs à esquerda. Observamos que tais restrições delimitam o espaço de busca de soluções para o último subMILP e auxiliam o Gurobi a encontrar melhores soluções. Para o conjunto Fdata, em 70\% das instâncias em LB_SD e em 30\% das instâncias em LB_CD, a solução ótima foi obtida em ambas as ramificações. 
Tabela 30 - Resultados computacionais de MILP-1-Gurobi, LB_SD e LB_CD para o conjunto BCdata.

\begin{tabular}{|c|c|c|c|c|c|c|}
\hline \multirow[t]{2}{*}{ Problema } & \multicolumn{2}{|c|}{ MILP-1-Gurobi } & \multicolumn{2}{|c|}{ LB_SD } & \multicolumn{2}{|c|}{ LB_CD } \\
\hline & $C_{\text {Max }}$ & Tempo(s) & $C_{\operatorname{Max}}$ & Tempo(s) & $C_{M a x}$ & Tempo(s) \\
\hline $\mathrm{mt10c1}$ & 927 & 43,7 & 927 & 818,72 & 927 & 3600 \\
\hline $\mathrm{mt} 10 \mathrm{cc}$ & 908 & 27,45 & 908 & 1389,94 & 919 & 3600 \\
\hline $\operatorname{mt} 10 \mathrm{x}$ & 918 & 50,41 & 918 & 1338,67 & 931 & 3600 \\
\hline $\operatorname{mt10xx}$ & 918 & 34,06 & 918 & 1217,52 & 929 & 3600 \\
\hline $\operatorname{mt} 10 \mathrm{xxx}$ & 918 & 34,82 & 918 & 637,80 & 926 & 3600 \\
\hline mt10xy & 905 & 23,87 & 905 & 794,64 & 905 & 3600 \\
\hline mt10xyz & 847 & 27,57 & 847 & 795,63 & 858 & 3600 \\
\hline setb4c9 & 914 & 156,7 & 914 & 1192,47 & 926 & 3600 \\
\hline setb4cc & 907 & 260,19 & 907 & 2434,30 & 922 & 3600 \\
\hline setb4x & 925 & 3600 & 925 & 3339,67 & 930 & 3600 \\
\hline setb4xx & 925 & 1210,55 & 925 & 3600 & 925 & 3600 \\
\hline setb4xxx & 925 & 3600 & 925 & 3600 & 929 & 3600 \\
\hline setb4xy & 910 & 688,26 & 910 & 1850,18 & 928 & 3600 \\
\hline setb4xyz & 902 & 136,39 & 902 & 237,25 & 914 & 3600 \\
\hline seti5c12 & 1180 & 3600 & 1182 & 3600 & 1188 & 3600 \\
\hline seti5cc & 1138 & 3600 & 1136 & 3600 & 1138 & 3600 \\
\hline seti5x & 1207 & 3600 & 1209 & 3600 & 1228 & 3600 \\
\hline seti5xx & 1200 & 3600 & 1211 & 3600 & 1220 & 3600 \\
\hline seti5xxx & 1205 & 3600 & 1206 & 3600 & 1228 & 3600 \\
\hline seti5xy & 1138 & 3600 & 1136 & 3600 & 1138 & 3600 \\
\hline seti5xyz & 1136 & 3600 & 1137 & 3600 & 1135 & 3600 \\
\hline
\end{tabular}

No trabalho de Danna, Rothberg e Pape (2005), os autores apontam que a qualidade da solução inicial utilizada para começar as matheuristics LB e RINS pode ser um fator importante na obtenção de bons resultados globais e na melhoria da qualidade da solução. Para isto, os autores utilizaram nas implementações duas soluções iniciais distintas: a primeira solução factível encontrada pelo resolvedor CPLEX e a obtida após uma hora de processamento.

Diante desta observação, repetimos os experimentos considerando uma nova solução como ponto de partida. Para estabelecer uma nova solução inicial, analisamos o comportamento da curva da média geométrica da razões para as 59 instâncias teste (Figura 20). Assim como no processo de calibração, a razão entre o melhor valor do limitante superior obtido até o presente momento e o melhor valor obtido no final da execução dos métodos MILP-Gurobi, LB_SD e LB_CD foi calculada a cada 100 segundos de processamento, durante 3600 segundos. Na curva da média geométrica do método MILP-1-Gurobi, observamos um maior decrescimento nos primeiros 700 minutos. Dessa forma, refizemos os experimentos nos métodos LB_SD e LB_CD, mas considerando a solução inicial como sendo a obtida pelo Gurobi após 300 segundos de processamento. Denominamos os novos procedimentos de LB_SD-300 e LB_CD-300. 
Tabela 31 - Resultados computacionais de MILP-1-Gurobi, LB_SD e LB_CD para o conjunto DPdata.

\begin{tabular}{|c|c|c|c|c|c|}
\hline \multirow[t]{2}{*}{ Problema } & \multicolumn{2}{|c|}{ MILP-1-Gurobi } & \multicolumn{2}{|c|}{ LB_SD } & $\mathrm{LB} \_\mathrm{CD}$ \\
\hline & $C_{\operatorname{Max}}$ & Tempo(s) & $C_{M a x}$ & Tempo(s) & $C_{\text {Max }}$ Tempo(s) \\
\hline $01 \mathrm{a}$ & 2929 & 3600 & 2767 & 3600 & 27623600 \\
\hline $02 \mathrm{a}$ & 2596 & 3600 & 2521 & 3600 & $2554 \quad 3600$ \\
\hline $03 a$ & 2359 & 3600 & 2538 & 3600 & $2538 \quad 3600$ \\
\hline $04 \mathrm{a}$ & 2713 & 3600 & 2768 & 3600 & 26773600 \\
\hline $05 a$ & 2464 & 3600 & 2396 & 3600 & 23763600 \\
\hline $06 \mathrm{a}$ & 2254 & 3600 & 2460 & 3600 & $2465 \quad 3600$ \\
\hline $07 a$ & 2665 & 3600 & 2688 & 3600 & $2708 \quad 3600$ \\
\hline $08 \mathrm{a}$ & 2265 & 3600 & 2360 & 3600 & 23693600 \\
\hline $09 \mathrm{a}$ & 2405 & 3600 & 2381 & 3600 & 28643600 \\
\hline $10 \mathrm{a}$ & 2794 & 3600 & 2732 & 3600 & $\mathbf{2 6 2 6} 3600$ \\
\hline $11 \mathrm{a}$ & 2332 & 3600 & 2403 & 3600 & $2390 \quad 3600$ \\
\hline $12 \mathrm{a}$ & 2552 & 3600 & 2500 & 3600 & 30643600 \\
\hline $13 \mathrm{a}$ & 2746 & 3600 & 2742 & 3600 & $\mathbf{2 7 0 8} 3600$ \\
\hline $14 \mathrm{a}$ & 2797 & 3600 & 2742 & 3600 & 24483600 \\
\hline $15 \mathrm{a}$ & - & - & - & - & - \\
\hline $16 \mathrm{a}$ & 2672 & 3600 & 2595 & 3600 & 25953600 \\
\hline $17 \mathrm{a}$ & 2696 & 3600 & 2786 & 3600 & $3335 \quad 3600$ \\
\hline $18 \mathrm{a}$ & - & - & 3539 & 3600 & $\mathbf{3 1 6 4} 3600$ \\
\hline
\end{tabular}

Assim, o novo valor de referência para o cálculo da solução inicial pode permitir que as soluções obtidas pelos subMILPs tenham valores melhores que a da solução inicial, uma vez que ainda há margem para melhoria. Se a solução inicial não for encontrada após o tempo limite de 300 segundos, o Gurobi continua o processamento até encontrar a primeira solução factível.

A tabela 32 apresenta a média dos gaps para cada procedimento e para cada estratégia de obtenção da solução inicial. O valor do gap é determinado por $100 \cdot\left(C_{\text {Max }}-\right.$ Melhor_C $\left.C_{\text {Max }}\right) /$ Melhor_C $C_{\text {Max }}$, em que Melhor_C $C_{\text {Max }}$ representa o melhor makespan entre os três procedimentos. Para a média dos gaps no conjunto DPdata, como não foi possível encontrar uma solução factível para as instâncias 15a e 18a em MILP-1-Gurobi, tais problemas não foram levados em consideração nos 3 procedimentos.

Conforme a tabela 32, o processo de utilizar a solução inicial após o tempo de 300 segundos melhorou, de forma significativa, a média dos gaps de LB_SD no conjunto DPdata e de LB_CD nos conjuntos BRdata e DPdata. Com a nova solução inicial, no conjunto BRdata, o procedimento MILP-1-Gurobi obteve, de forma isolada, o melhor gap somente para a instância MK05. Ainda no conjunto BRdata, as abordagens LBs melhoram a solução em relação a MILP-1-Gurobi em 4 instâncias. Para as demais instâncias de BRdata, a melhor solução foi obtida por MILP-1-Gurobi e, no mínimo, por um dos procedimentos LBs. Em DPdata, de um total de 18 instâncias, LB_SD e LB_CD superaram o valor da solução de MILP-1-Gurobi em 15 delas, além de encontrarem um limitante superior para o makespan 


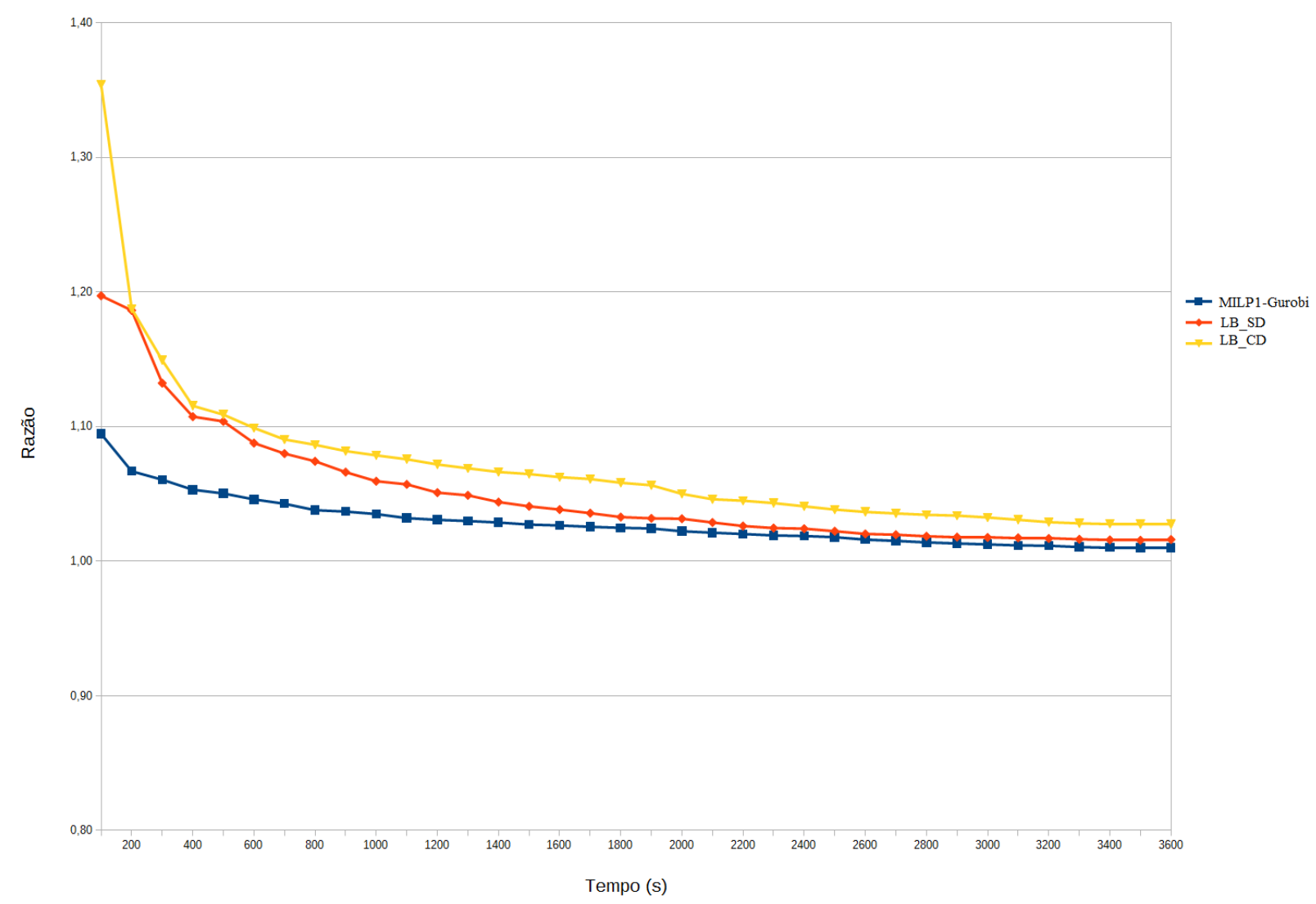

Figura 20 - Resolução da instância 02a do conjunto DPdata

Tabela 32 - Valor da média do GAP para cada procedimento e estratégia de obtenção da solução inicial

\begin{tabular}{|c|c|c|c|}
\hline & MILP-1-Gurobi & LB_SD & LB_CD \\
\hline Iniciando na segunda solução factível & GAP $(\%)$ & GAP $(\%)$ & GAP $(\%)$ \\
\hline Fdata (mfs01-mfs10) & 0,00 & 0,15 & 0,02 \\
\hline BRdata & 1,57 & 1,01 & 5,27 \\
\hline BCdata & 0,02 & 0,07 & 0,93 \\
\hline DPdata & 2,64 & 2,93 & 5,84 \\
\hline Iniciando após 300 segundos & $\operatorname{GAP}(\%)$ & GAP $(\%)$ & GAP $(\%)$ \\
\hline Fdata (mfs01-mfs10) & 0,13 & 0,19 & 0,13 \\
\hline BRdata & 1,78 & 1,00 & 2,04 \\
\hline BCdata & 0,08 & 0,12 & 0,39 \\
\hline DPdata & 0,02 & 0,02 & 0,13 \\
\hline
\end{tabular}

no problema 15a. De forma geral, ao considerar a escolha de uma melhor solução inicial, o número de instâncias em que as soluções de LB_SD ou LB_CD melhoraram a solução de MILP-1-Gurobi aumentou de 14 instâncias para 26 problemas. Além disso, das 59 instâncias teste, o valor da solução de MILP-1 foi estritamente menor em relação às soluções de LB_SD ou LB_CD em somente 5 instâncias (mfjs10, Mk05, 06a, 08a e 17a). 


\subsubsection{Experimentos computacionais para método DRT}

Nesta seção, apresentamos os resultados obtidos pela implementação de duas abordagens que utilizam os princípios do método DRT. A primeira abordagem, denominada de DRT1, é uma variação do DRT tradicional. Nesta variante, foram alteradas as restrições de diversificação e ajuste-refinamento. Na segunda abordagem, utilizamos o teorema 6 da seção 2.3.1 para selecionar as variáveis binárias que compõem as vizinhanças em cada uma das etapas do método. Denominamos este procedimento de DRT2. Na sequência, descrevemos com mais detalhes cada uma destas abordagens e os valores utilizados em seus parâmetros.

Conforme o algoritmo 2, o método DRT possui muitos parâmetros de entrada e, para definir seus valores, levamos em consideração as observações extraídas dos experimentos iniciais e dos valores adotados no trabalho de Fischetti, Polo e Scantamburlo (2004). Os parâmetros gerais do método englobam o tempo total de processamento, o tempo de processamento para obter a primeira solução e a ênfase na estratégia para obter a solução factível. O tempo de processamento para execução de cada instância teste é limitado em 3600 segundos. Tomando como referência a segunda parte dos experimentos computacionais do método LB (seção 4.4.1), a solução inicial para o DRT1 é a obtida pelo Gurobi após 300 segundos de processamento. Se nenhuma solução for encontrada, o Gurobi continua o processamento até encontrar a primeira solução factível. Além disso, no cálculo da solução inicial e nas etapas do DRT1, configuramos o Gurobi, através do parâmetro MIPFocus, para dar mais ênfase na factibilidade da solução menos ênfase na análise e prova de sua otimalidade (MIPFocus $=1$ ).

Além de parâmetros gerais, o DRT possui um conjunto de parâmetros para cada etapa do método. A etapa de diversificação tem os parâmetros $k_{1}^{\text {ini }}$ e $k_{1}^{\text {passo }}$. Nesta etapa, busca-se uma nova solução de referência próxima da solução corrente acrescentando temporariamente a restrição (4.6) ao modelo. Devido à natureza combinatória do JSF, mesmo configurando $k_{1}^{\min }=1$ e $k_{1}^{\max }=2$, observamos em muitas instâncias que o valor da nova solução é muito superior ao valor da solução corrente, mesmo a restrição (4.6) indicando que o número de trocas de valores da variável de primeiro nível é baixo. Em muitos casos, como o valor da nova solução inicial é cinco vezes maior que o valor da solução corrente, uma parte significativa do tempo destinado à etapa de refinamento é utilizado para recuperar o valor perdido na solução corrente. Portanto, para restringir a vizinhança da solução corrente em nosso algoritmo, substituímos a restrição de diversificação (4.6) por

$$
k_{1}^{\min } \leq \Delta(x, \bar{x}) \leq k_{1}^{\max }
$$

em que

$$
\Delta(x, \bar{x})=\sum_{j \in \mathscr{B}_{1} \cup \mathscr{B}_{2} ; \bar{x}_{j}=1}\left(1-x_{j}\right)+\sum_{j \in \mathscr{B}_{1} \cup \mathscr{B}_{2} ; \bar{x}_{j}=0} x_{j}
$$

Assim, a nova restrição de diversificação (4.8) é formada pelas variáveis binárias de primeiro e segundo níveis. Além disso, definimos $k_{1}^{\text {min }}=1$ e $k_{1}^{\text {passo }}=4$.

Conforme comentado no início desta seção, os autores do método DRT estabelecem que o parâmetro $k_{1}$ presente no lado direito da restrição local branching (4.4) deve ser um valor baixo, como por exemplo $k_{1} \in\{0,1,2\}$. Nos experimentos iniciais, observamos que, para tais valores de referência, havia pouca redução do limitante superior. Dessa forma, em DRT1 definimos para $k_{1}$ o valor 8 .

Também observamos pouca evolução na qualidade da solução corrente na etapa de ajuste-refinamento. Nesta etapa, para explorar uma maior vizinhança para as variáveis do 
segundo nível, substituímos a restrição (4.5) por

$$
k_{2}^{\min } \leq \Delta_{2}(x, \bar{x}) \leq k_{2}^{\max }
$$

em que $k_{2}^{\max }=k_{2}^{\min }+k_{2}^{\text {passo }}$, e definimos $k_{2}^{\min }=1$ e $k_{2}^{\text {passo }}=3$. O processo de ajusterefinamento é finalizado quando o tempo destinado a esta etapa atingir o valor limite ou quando $k_{2}^{\min }>17$.

Outro importante parâmetro está relacionado ao tempo de execução de cada etapa do DRT. Se o tempo para a etapa de refinamento for alto, então o método irá explorar as variáveis do primeiro nível com maior intensidade. Dessa forma, o método terá poucas etapas de diversificação. Conforme Fischetti, Polo e Scantamburlo (2004), dependendo das características do problema, pode-se ter um melhor desempenho do método atribuindo um maior número de diversificações e um menor limite de tempo para as etapas de refinamento e ajustorefinamento. Nos problemas analisados pelos autores, dentro do limite de 3 horas de execução para cada instância, 5 minutos (2,78\% do tempo total) são destinados para cada etapa de refinamento e 3 minutos (1,68\% do tempo total) para cada etapa de ajuste-refinamento. Com base nos experimentos iniciais, observamos que melhores resultados são obtidos destinando uma maior porcentagem para a etapa de refinamento e ajuste-refinamento. Dessa forma, em DRT1, definimos o tempo de 450 segundos para os parâmetros time_limit_ref e total_time_limit_ajus_ref e de 300 segundos para time_limit_diver. Além disso, através do parâmetro time_limit_ajus_ref, definimos o limite de tempo de execução do Gurobi para cada configuração de $\bar{k}_{2}^{\min }$ e $k_{2}^{\max }$ em 110 segundos. Dessa forma, se o método executar a fase de ajuste-refinamento, serão explorados ao menos 4 configurações distintas para os parâmetros $k_{2}^{\min }$ e $k_{2}^{\max }$.

Uma segunda versão do algoritmo DRT foi implementada tendo como motivação o teorema 6. Denominamos esta versão de DRT2. O referido teorema estabelece que, para reduzir o makespan de um sequenciamento, ou é preciso mudar a atribuição de alguma operação de um bloco crítico para outra máquina, ou pelo menos uma operação de algum bloco tem que ser processada antes da primeira ou após a última operação do respectivo bloco.

Assim como é possível expressar certas propriedades das metaheurísticas por meio de expressões ou inequações matemáticas, em DTR2 procuramos representar a propriedade do teorema através de inequações, mais precisamente, por meio das restrições local branching. Dessa forma, as variáveis de primeiro e de segundo níveis que compõem as restrições local branching são definidas pelas operações que fazem parte dos blocos do caminho crítico.

Para descrever as novas restrições, considere $\bar{z}$ uma solução factível para o modelo MILP-1. Observe que $\bar{z}$ é formada pela variável contínua $s_{i}$, pelas variáveis binárias $x_{i k}$ e $y_{i j}$, e pelas variáveis $p_{i}$ e $C_{\text {Max }}$. Seja $P_{(\mu, S)}$ o caminho crítico do grafo $G_{(\mu, S)}$ associado à solução $\bar{z}$. Suponha que $P_{(\mu, S)}$ seja composto pelos blocos $B_{d}\left(\left|B_{d}\right|>2\right), d=1, \ldots, p$. Além disso, sejam $f_{p}$ e $l_{p}$ a primeira e a última operação de cada bloco $B_{d}$, respectivamente.

Em DRT2, a restrição local branching que define a mudança de estado para as variáveis do primeiro nível será formada apenas pelas variáveis binárias $x_{i k}$, tal que $i \in B_{d}$. Portanto, $\Delta_{1}(z, \bar{z})$ é definida por

$$
\Delta_{1}(z, \bar{z})=\sum_{d=1}^{p} \sum_{k=1}^{m} \sum_{\substack{i \in B_{d} \cap \mathcal{O}_{k} \\ \bar{x}_{i k}=1}}\left(1-x_{i k}\right)+\sum_{d=1}^{p} \sum_{k=1}^{m} \sum_{\substack{i \in B_{d} \cap \mathcal{O}_{k} \\ \bar{x}_{i k}=0}} x_{i k}
$$


Para as de segundo nível, a restrição local branching será formada pelas variáveis binárias $y_{i j}$, tal que $i$ e $j \in B_{d}$. Além disso, a mudança de estado é aplicada somente para as operações que precedem a primeira operação do bloco e para as que sucedem a última operação do bloco. Dessa forma, $\Delta_{2}(z, \bar{z})$ é definida por

$$
\Delta_{2}(z, \bar{z})=\sum_{d=1}^{p} \sum_{\substack{i \in B_{d} \\ i \neq f_{d}}} y_{i f_{d}}+\sum_{d=1}^{p} \sum_{\substack{i \in B_{d} \\ i \neq l_{d}}} y_{l_{d} i}
$$

Se considerarmos a restrição tabu $\Delta_{1}(z, \bar{z}) \geq 1$, então pelo menos uma operação pertencente a algum bloco crítico deverá ser atribuída a uma outra máquina. Pelo teorema 6 , este fato não necessariamente poderá ocorrer, uma vez que poderá haver a troca na ordem de execução entre a primeira ou a última operação de algum bloco crítico. Dessa forma, a restrição tabu no método DRT2 é dada pela inequação

$$
\Delta(z, \bar{z})=\Delta_{1}(z, \bar{z})+\Delta_{2}(z, \bar{z}) \geq 1
$$

Os blocos críticos são definidos pelo caminho crítico do grafo disjuntivo associado à solução corrente. O algoritmo que determina o caminho crítico utiliza a propriedade de que se $i$ e $j$ são duas operações consecutivas no caminho crítico, então o comprimento entre $i$ e $j$ é dado pelo tempo de processamento da operação $i$ (NAJID; DAUZERE-PÉRÈS; ZAIDAT, 2002). Assim, o valor do caminho crítico é dado pela soma do tempo de processamento de suas operações. Dessa forma, dado o valor do makespan da solução corrente, o algoritmo identifica as operações do caminho crítico através de sua ordem inversa. Além disso, caso seja encontrado mais de um caminho crítico, selecionamos o que possui a maior quantidade de operações.

Com a nova definição, as restrições presentes nas fases de diversificação, de refinamento e de ajuste-refinamento são descritas por

$$
\begin{gathered}
k_{1}^{\min } \leq \Delta_{1}(z, \bar{z}) \leq k_{1}^{\max }, \\
\Delta_{1}(z, \bar{z}) \leq k_{1}^{\max } \\
k_{2}^{\min } \leq \Delta_{2}(z, \bar{z}) \leq k_{2}^{\max },
\end{gathered}
$$

respectivamente.

As tabelas 33, 34, 35 e 36 comparam o desempenho dos métodos DRT1 e DRT2 com o MILP-1-Gurobi. As colunas de cada tabela descrevem o nome da instância teste e o valor do makespan em cada uma das abordagens. Em cada instância de teste, destacamos o melhor valor do makespan quando este for obtido, no máximo, por duas abordagens.

Os resultados mostram que MILP-1-Gurobi obteve, de forma isolada, a melhor solução para 14 das instâncias de teste, o que representa 23,73\% do total. As soluções em DRT1 e DRT2 superam as obtidas por MILP-1-Gurobi em 19 das instâncias (32,20\%). Deste total, 14 das melhores soluções foram obtidas por DRT1, 4 por DRT2 e 1 por ambos os métodos (instância 18a do conjunto DPdata). Os três métodos não alcançaram uma solução factível para a instância 15a do conjunto DPdata. Para as demais 25 instâncias, o valor obtido com MILP-1-Gurobi coincide com DRT1 ou DRT2.

Com exceção das instâncias mfjs01-mfjs07 do conjunto Fdata, o tempo de processamento dos métodos DRT1 e DRT2 em cada instância foi de 3600 segundos. Para as instâncias 
Tabela 33 - Resultados computacionais de MILP-1-Gurobi, DRT1 e DRT2 para o conjunto Fdata.

\begin{tabular}{|c|c|c|c|}
\hline \multirow[t]{2}{*}{ Problema } & MILP-1-Gurobi & DRT1 & DRT2 \\
\hline & $C_{\operatorname{Max}}$ & $C_{\text {Max }}$ & $C_{M a x}$ \\
\hline mfjs01 & 468 & 468 & 468 \\
\hline mfjs02 & 446 & 446 & 446 \\
\hline mfjs03 & 466 & 466 & 466 \\
\hline mfjs04 & 554 & 554 & 554 \\
\hline mfjs 05 & 514 & 514 & 514 \\
\hline mfjs06 & 634 & 634 & 634 \\
\hline mfjs07 & 879 & 879 & 879 \\
\hline mfjs08 & 884 & 884 & 884 \\
\hline mfjs09 & 1073 & 1070 & 1093 \\
\hline mfjs10 & 1214 & 1225 & 1208 \\
\hline
\end{tabular}

Tabela 34 - Resultados computacionais de MILP-1-Gurobi, DRT1 e DRT2 para o conjunto BRdata.

\begin{tabular}{|c|c|c|c|}
\hline \multirow[t]{2}{*}{ Problema } & MILP-1-Gurobi & DRT1 & DRT2 \\
\hline & $C_{M a x}$ & $C_{M a x}$ & $C_{M a x}$ \\
\hline Mk01 & 40 & 40 & 40 \\
\hline $\mathrm{Mk} 02$ & 26 & 26 & 26 \\
\hline Mk03 & 204 & 204 & 213 \\
\hline Mk04 & 60 & 63 & 63 \\
\hline Mk05 & 177 & 177 & 176 \\
\hline Mk06 & 70 & 65 & 73 \\
\hline Mk07 & 170 & 150 & 156 \\
\hline Mk08 & 524 & 524 & 533 \\
\hline Mk09 & 360 & 341 & 354 \\
\hline Mk10 & 250 & 261 & 250 \\
\hline
\end{tabular}

mfjs01-mfjs07, a solução ótima foi obtida em menos de 300 segundos, tempo este destinado para obter a solução inicial nos métodos DRT1 e DRT2.

Considerando o método DRT1, em 19 instâncias teste a melhor solução foi obtida na fase de refinamento e para 5 instâncias a melhor solução foi alcançada na etapa de ajuste-refinamento. Em 25 casos, a melhor solução foi obtida em ambas as etapas. Para as instâncias $14 \mathrm{a}$ e $18 \mathrm{a}$ do conjunto DCdata, o valor $C_{M a x}$ se refere ao obtido pela solução inicial, uma vez que o tempo de processamento destinado a cada uma das etapas do método DRT1 foi insuficiente para produzir uma solução factível. Para o método DRT2, a melhor solução foi obtida na fase de refinamento em 17 instâncias e na fase de ajuste-refinamento em 13 instâncias. Assim como em DRT1, o tempo destinado a cada etapa do método foi insuficiente para produzir uma solução factível para as instâncias 14a, 09a, 12a e 18a. Além 
Tabela 35 - Resultados computacionais de MILP-1-Gurobi, DRT1 e DRT2 para o conjunto BCdata.

\begin{tabular}{|c|c|c|c|}
\hline \multirow[t]{2}{*}{ Problema } & MILP-1-Gurobi & DRT1 & DRT2 \\
\hline & $C_{\text {Max }}$ & $C_{M a x}$ & $C_{\operatorname{Max}}$ \\
\hline mt10c1 & 927 & 927 & 927 \\
\hline mt10cc & 908 & 908 & 908 \\
\hline $\operatorname{mt} 10 \mathrm{x}$ & 918 & 918 & 918 \\
\hline $\operatorname{mt10xx}$ & 918 & 918 & 918 \\
\hline $\operatorname{mt10xxx}$ & 918 & 918 & 918 \\
\hline mt10xy & 905 & 905 & 905 \\
\hline mt10xyz & 847 & 849 & 847 \\
\hline setb4c9 & 914 & 919 & 919 \\
\hline setb4cc & 907 & 907 & 907 \\
\hline setb4x & 925 & 925 & 925 \\
\hline setb4xx & 925 & 925 & 925 \\
\hline setb4xxx & 925 & 925 & 925 \\
\hline setb4xy & 910 & 916 & 915 \\
\hline setb4xyz & 902 & 905 & 908 \\
\hline seti5c12 & 1180 & 1174 & 1179 \\
\hline seti5cc & 1138 & 1136 & 1138 \\
\hline seti5x & 1207 & 1215 & 1215 \\
\hline seti5xx & 1200 & 1202 & 1202 \\
\hline seti5xxx & 1205 & 1202 & 1204 \\
\hline seti5xy & 1138 & 1136 & 1138 \\
\hline seti5xyz & 1136 & 1134 & 1130 \\
\hline
\end{tabular}

disso, em 6 instâncias (mfjs09, Mk02, Mk06, seti5xx, 02a e 05a), embora o método tenha alcançado outras soluções factíveis durante o tempo de processamento, a melhor solução foi produzida pela solução factível inicial. Por fim, o valor do $C_{M a x}$ foi obtido em ambas as etapas em 11 instâncias. Esses resultados mostram a importância de explorar as vizinhanças definidas pelas variáveis de primeiro e segundo níveis, uma vez que a solução de diversas instâncias ocorrem em uma destas regiões.

Fischetti e Lodi desenvolveram uma variação do DRT que tem como característica um mecanismo de grande diversificação. A etapa de grande diversificação consiste em ampliar o número de mudanças de estado das variáveis de primeiro nível na restrição (4.6) tão logo o algoritmo identifique a estagnação da solução corrente. Para que isso aconteça, assim que o número máximo de diversificação sem melhoria é atingido, o algoritmo define temporariamente um novo e maior valor para os parâmetros $k_{1}^{\text {min }}$ e $k_{1}^{\text {passo }}$.

Implementamos o método DRT1 com o mecanismo de grande diversificação (DRT1Big). Para isso, se o algoritmo identificar em três iterações seguidas que o gap entre a solução corrente e solução incumbente é superior a 0,05, então aplica-se a etapa de grande diversificação. Caso contrário, aplica-se a etapa tradicional de diversificação. Dessa forma, as etapas de grande diversificação e diversificação diferem apenas nos valores dos parâmetros 
Tabela 36 - Resultados computacionais de MILP-1-Gurobi, DRT1 e DRT2 para o conjunto DPdata.

\begin{tabular}{|c|c|c|c|}
\hline \multirow[t]{2}{*}{ Problema } & \multirow{2}{*}{$\frac{\text { MILP-1-Gurobi }}{C_{\text {Max }}}$} & \multirow{2}{*}{$\frac{\mathrm{DRT} 1}{C_{\text {Max }}}$} & \multirow{2}{*}{$\begin{array}{l}\mathrm{DRT} 2 \\
C_{\operatorname{Max}}\end{array}$} \\
\hline & & & \\
\hline $01 a$ & 2929 & 2764 & 2834 \\
\hline $02 \mathrm{a}$ & 2596 & 2432 & 2447 \\
\hline $03 a$ & 2359 & 2402 & 2406 \\
\hline $04 a$ & 2713 & 2727 & 2670 \\
\hline $05 a$ & 2464 & 2460 & 2480 \\
\hline $06 a$ & 2254 & 2438 & 2366 \\
\hline $07 \mathrm{a}$ & 2665 & 2705 & 2735 \\
\hline $08 \mathrm{a}$ & 2265 & 2356 & 2320 \\
\hline 09a & 2405 & 2452 & 16485 \\
\hline $10 \mathrm{a}$ & 2794 & 2693 & 2792 \\
\hline $11 \mathrm{a}$ & 2332 & 2382 & 2379 \\
\hline $12 \mathrm{a}$ & 2552 & 2550 & 2856 \\
\hline $13 \mathrm{a}$ & 2746 & 2709 & 2884 \\
\hline $14 \mathrm{a}$ & 2797 & 3472 & 3472 \\
\hline $15 \mathrm{a}$ & - & - & - \\
\hline $16 \mathrm{a}$ & 2672 & 2686 & 2784 \\
\hline $17 \mathrm{a}$ & 2696 & 2788 & 3037 \\
\hline $18 \mathrm{a}$ & - & 3164 & 3164 \\
\hline
\end{tabular}

$k_{1}$ 's.

Para a etapa de grande diversificação, definimos $k_{1}^{\text {ini }}=15$ e $k_{1}^{\text {passo }}=10$. Para os demais parâmetros, foram utilizados os mesmos valores de DRT1. Comparado com o DRT1, observamos que a nova proposta de diversificação não alterou o valor do makespan em todas as instâncias dos conjuntos Fdata, BRdata e BCdata. O valor do makespan foi alterado somente para seis instâncias do conjunto DPdata. A tabela 37 apresenta os resultados obtidos por estas seis instâncias.

Tabela 37 - Resultados computacionais de DRT1-Big.

\begin{tabular}{|c|c|c|}
\hline \multirow[t]{2}{*}{ Problema } & DRT1 & DRT1-Big \\
\hline & $C_{M a x}$ & $C_{\text {Max }}$ \\
\hline $02 \mathrm{a}$ & 2432 & 2424 \\
\hline $04 a$ & 2727 & 2731 \\
\hline $08 \mathrm{a}$ & 2356 & 2354 \\
\hline $11 \mathrm{a}$ & 2382 & 2368 \\
\hline $12 \mathrm{a}$ & 2550 & 2493 \\
\hline $17 \mathrm{a}$ & 2788 & 2879 \\
\hline
\end{tabular}


Conforme os resultados descritos na tabela 37, o DRT1-Big melhorou o makespan para 4 instâncias, e dentre estes valores, 02a e 12a foram melhores do que em MILP-1-Gurobi.

\subsubsection{Síntese dos experimentos para os métodos local branching e DRT}

Para sintetizar os resultados obtidos nos experimentos anteriores, nesta seção comparamos o desempenho do resolvedor Gurobi, das abordagens LB com e sem a etapa de disversificação (LB_SD e LB_CD), com a nova solução factível (LB_SD-300 e LB_CD-300) e das abordagens DRT1, DRT1-Big e DRT2. Para cada instância, descrevemos o melhor valor obtido para o makespan e o método que teve o melhor desempenho. Os resultados estão descritos nas tabelas 38, 39, 40 e 41 .

Das 59 instâncias analisadas, somente em 3 problemas (6a, 8a e 17a) a melhor solução foi obtida, com exclusividade, pelo Gurobi. Em 28 instâncias, o menor valor para o makespan foi obtido somente pelas matheuristics LB e DRT. Este total equivale a 47,46\% do total das instâncias analisadas. Acreditamos que este percentual representa um bom desempenho das técnicas matheuristics utilizadas nesta tese. Para as instâncias restantes, a melhor solução foi obtida por MILP-1-Gurobi e por, ao menos, uma das variações de LB ou DRT.

Tabela 38 - Melhores resultados para as instâncias do conjunto Fdata

\begin{tabular}{lll}
\hline Problema & $C_{\text {Max }}$ & \multicolumn{1}{c}{ Método } \\
\hline mfjs01 & 468 & Todos \\
mfjs02 & 446 & Todos \\
mfjs03 & 466 & Todos \\
mfjs04 & 554 & Todos \\
mfjs05 & 514 & Todos \\
mfjs06 & 634 & Todos \\
mfj07 & 879 & Todos \\
mfjs08 & 884 & Todos \\
mfjs09 & 1059 & LB_CD-300 \\
mfjs10 & 1208 & DRT2 \\
\hline
\end{tabular}

Tabela 39 - Melhores resultados para as instâncias do conjunto BRdata

\begin{tabular}{lll}
\hline Problema & $C_{\text {Max }}$ & \\
\hline Mk01 & 40 & Todos \\
Mk02 & 26 & MILP-1-Gurobi, LB_CD, LB_CD-300, DRT1, DRT2 e DRT1-Big \\
Mk03 & 204 & MILP-1-Gurobi, LB_SD, LB_SD-300, LB_CD, LB_CD-300, DRT1 e DRT1-Big \\
Mk04 & 60 & MILP-1-Gurobi, LB_SD e LB_SD-300 \\
Mk05 & 176 & DRT2 \\
Mk06 & 65 & DRT1 e DRT1-Big \\
Mk07 & 150 & DRT1 e DRT1-Big \\
Mk08 & 524 & MILP-1-Gurobi, LB_SD, LB_SD-300, LB_CD, DRT1 e DRT1-Big \\
Mk09 & 341 & DRT1 e DRT1-Big \\
Mk10 & 250 & LB_SD-300 \\
\hline
\end{tabular}


Tabela 40 - Melhores resultados para as instâncias do conjunto BCdata

\begin{tabular}{lll}
\hline Problema & $C_{M a x}$ & \multicolumn{1}{c}{ Método } \\
\hline mt10c1 & 927 & Todos \\
mt10cc & 908 & MILP-1-Gurobi, LB_SD, LB_SD-300, LB_CD-300, DRT1, DRT2 e DRT1-Big \\
mt10x & 918 & MILP-1-Gurobi, LB_SD, LB_SD-300, LB_CD-300, DRT1, DRT2 e DRT1-Big \\
mt10xx & 918 & MILP-1-Gurobi, LB_SD, LB_SD-300, DRT1, DRT2 e DRT1-Big \\
mt10xxx & 918 & MILP-1-Gurobi, LB_SD, LB_SD-300, LB_CD-300, DRT1, DRT2 e DRT1-Big \\
mt10xy & 905 & MILP-1-Gurobi, LB_SD, LB_SD-300, LB_CD, DRT1, DRT2 e DRT1-Big \\
mt10xyz & 847 & MILP-1-Gurobi, LB_SD, LB_SD-300 e DRT2 \\
setb4c9 & 914 & MILP-1-Gurobi, LB_SD e LB_SD-300 \\
setb4cc & 907 & MILP-1-Gurobi, LB_SD, LB_SD-300, DRT1, DRT2 e DRT1-Big \\
setb4x & 925 & MILP-1-Gurobi, LB_SD, LB_SD-300, DRT1, DRT2 e DRT1-Big \\
setb4xx & 925 & MILP-1-Gurobi, LB_SD, LB_SD-300, LB_CD, DRT1, DRT2 e DRT1-Big \\
setb4xxx & 925 & MILP-1-Gurobi, LB_SD, LB_SD-300, LB_CD-300, DRT1, DRT2 e DRT1-Big \\
setb4xy & 910 & MILP-1-Gurobi, LB_SD e LB_SD-300 \\
setb4xyz & 902 & MILP-1-Gurobi, LB_SD e LB_SD-300 \\
seti5c12 & 1174 & DRT1 e DRT1-Big \\
seti5cc & 1136 & LB_SD, LB_CD-300, DRT1 e DRT1-Big \\
seti5x & 1205 & LB_CD-300 \\
seti5xx & 1200 & MILP-1-Gurobi e LB_CD-300 \\
seti5xxx & 1200 & LB_CD-300 \\
seti5xy & 1136 & LB_SD, LB_CD-300, DRT1 e DRT1-Big \\
seti5xyz & 1129 & LB_SD \\
\hline
\end{tabular}

\subsection{Resumo}

Neste capítulo, apresentamos o segundo conjunto de estratégias de resolução do JSF. As abordagens utilizadas foram a local branching e a diversification, refining and tight-refining. Estas abordagens, denominadas de matheuristics, incorporam conceitos presentes na busca local durante a resolução do MILP.

Os resultados obtidos nos experimentos computacionais destacam o bom desempenho das estratégias utilizadas, quando comparado como o modelo MILP-1. De um total de 59 instâncias, somente em 3 problemas a resolução do modelo MILP-1-Gurobi obteve melhores resultados do que as abordagens analisadas. Porém, assim como no algoritmo B\&C, para os problemas em abertos as matheuristics não obtiveram o valor ótimo ou melhoram os valores dos limitantes. 
Tabela 41 - Melhores resultados para as instâncias do conjunto DPdata

\begin{tabular}{lll}
\hline Problema & $C_{M a x}$ & Método \\
\hline 01a & 2762 & LB_CD \\
02a & 2424 & DRT1-Big \\
03a & 2355 & LB_SD-300 \\
04a & 2670 & DRT2 \\
05a & 2376 & LB_CD \\
06a & 2254 & MILP-1-Gurobi \\
07a & 2595 & LB_SD-300 \\
08a & 2265 & MILP-1-Gurobi \\
09a & 2378 & LB_CD-300 \\
$10 \mathrm{a}$ & 2585 & LB_SD-300 e LB_CD-300 \\
$11 \mathrm{a}$ & 2229 & LB_CD-300 \\
$12 \mathrm{a}$ & 2492 & LB_SD-300 \\
13a & 2708 & LB_CD \\
14a & 2448 & LB_CD \\
$15 \mathrm{a}$ & 3287 & LB_CD-300 \\
16a & 2595 & LB_SD e LB_CD \\
17a & 2696 & MILP-1-Gurobi \\
18a & 3164 & LB_SD, LB_CD, LB_CD-300, DRT1, DRT2 e DRT1-Big \\
\hline
\end{tabular}




\section{Capítulo 5 Conclusão}

\subsection{Considerações finais}

Nesta tese empregamos duas estratégias para resolver o JSF. A primeira estratégia utilizou novas inequações válidas para o JSF em um algoritmo B\&C e a segunda duas técnicas matheuristics. As estratégias foram comparadas com a formulação de Birgin et al. (2014) (MILP-1) usando diversos conjuntos de instâncias da literatura.

Propomos oito classes de inequações válidas para o JSF, derivadas do POL e do JS. As inequações apresentadas são válidas para modelos que utilizam a variável de precedência de Manne (1960). Além disso, para as classes derivadas do JS, o modelo deve satisfazer a restrição do tipo $y_{i j k}+y_{j i k}=x_{i k} \cdot x_{j k}$. Caso contrário, a solução do modelo representa um limitante superior para o makespan ótimo. Dessa forma, no algoritmo B\&C utilizamos uma reformulação de MILP-1 (MILP-2) considerando a linearização da equação $y_{i j k}+y_{j i k}=x_{i k} \cdot x_{j k}$.

Analisamos 69 instâncias, distribuídas em 4 conjuntos. Observamos o comportamento de todas as classes de inequações válidas em um algoritmo de $\mathrm{PC}$ e, por tornarem a relaxação do modelo "mais justa", as classes Half Cuts, Reverse Half Cuts, Late Jobs e Reverse Late Jobs foram utilizadas em um algoritmo B\&C. Os experimentos computacionais mostraram que nosso algoritmo B\&C melhorou o gap para 37,68\% das instâncias analisadas. O B\&C reduziu o número médio de nós explorados na árvore de busca em cada conjunto de instância teste. Além disso, o gap médio também foi reduzido nos conjuntos BRdata (de 43,05\% para 13,53\%), BCdata (de 3,86\% para 0,90\%) e DPdata (de 45,75\% para 33,58\%). As estratégias utilizadas pelos algoritmos de separação foram eficientes, pois consumiram pouco tempo de processamento.

No segundo conjunto de estratégias, foram consideradas as implementações das matheuristics LB e DRT. Os experimentos computacionais analisaram o resultado de 59 instâncias. Para a técnica LB, inicialmente implementamos dois algoritmos: LB sem diversificação (LB_SD) e LB com diversificação (LB_CD). As soluções obtidas pelas duas abordagens superaram as obtidas por MILP-1-Gurobi em 23,73\% dos problemas analisados. Os resultados conquistados por LB_SD e LB_CD foram mais significativos considerando a estratégia de utilizar uma solução inicial de melhor qualidade. Nesta configuração, LB_SD ou LB_CD melhoraram a solução de MILP-1-Gurobi para 44,07\% dos problemas analisados e, para somente 8,47\% das instâncias, a melhor solução foi obtida por MILP-1-Gurobi.

O método DRT foi implementado em três variações: DRT1, DRT2 e DRT1-Big. No algoritmo DRT1, alteramos as variáveis que compõem a restrição de diversificação e ampliamos a vizinhança definida pela restrição de ajuste-refinamento. O algoritmo DRT2 
foi implementado levando em consideração algumas propriedades do problema. As soluções obtidas em DRT1 e DRT2 superaram as obtidas por MILP-1-Gurobi em 32,20\% dos casos analisados, sendo DRT1 o de melhor desempenho. O resultado de MILP-1-Gurobi superaram a DRT1 ou DRT2 em 23,73\% do total de instâncias. A estratégia de grande diversificação, aplicada em DRT1, melhorou o makespan para 6 instâncias.

De forma geral, as abordagens matheuristics tiveram um excelente desempenho. Somente em 3 problemas a resolução do modelo MILP-1-Gurobi obteve melhores soluções do que as abordagens matheuristcs. Em 47,46\% do total das instâncias analisadas, o menor valor para o makespan foi obtido somente pelas matheuristics LB ou DRT.

\subsection{Trabalhos futuros}

Mesmo com os bons resultados obtidos nesta tese, acreditamos que algumas melhorias podem ser alcanças. Um possível trabalho futuro consiste na implementação própria do algoritmo B\&C. Dessa forma, será possível analisar as diferentes estratégias de ramificação presentes na literatura, e aplicar por mais de uma vez o algoritmo de PC no subproblema associado a um determinado nó. Devido à utilização das bibliotecas do Gurobi, o nosso algoritmo B\&C limitou-se ao uso do algoritmo de PC em um única iteração no nó selecionado.

Além disso, as heurísticas utilizadas pelo Gurobi não produzem boas soluções iniciais para o JSF. Por exemplo, considerando a instância 15a do conjunto DPdata, após uma hora de processamento, o Gurobi não conseguiu obter nenhuma solução factível. Dessa forma, pretendemos utilizar heurísticas para construir, de preferência, boas soluções iniciais e dar atenção para os problemas em aberto. Posteriormente, vislumbramos combinar o algoritmo $\mathrm{B} \& \mathrm{C}$ com as abordagens matheuristics. 


\section{Referências}

ACHTERBERG, T.; KOCH, T.; MARTIN, A. Branching rules revisited. Operations Research Letters, Elsevier, v. 33, n. 1, p. 42-54, 2005.

AHO, A. V.; GAREY, M. R.; ULLMAN, J. D. The transitive reduction of a directed graph. SIAM Journal on Computing, SIAM, v. 1, n. 2, p. 131-137, 1972.

APPLEGATE, D.; COOK, W. A computational study of the job-shop scheduling problem. ORSA Journal on Computing, v. 3, n. 2, p. 149-156, 1991.

ARCHETTI, C.; SPERANZA, M. G. A survey on matheuristics for routing problems. EURO Journal on Computational Optimization, Springer, v. 2, n. 4, p. 223-246, 2014.

ARENALES, M. et al. Pesquisa operacional: para cursos de engenharia. 4 . ed. Rio de Janeiro: Elsevier, 2007.

BAKER, K. R.; TRIETSCH, D. Principles of sequencing and scheduling. [S.l.]: John Wiley \& Sons, 2013.

BALAS, E. On the facial structure of scheduling polyhedra. In: Mathematical Programming Essays in Honor of George B. Dantzig Part I. [S.l.]: Springer, 1985. p. 179-218.

BARNES, J. B. C. J. W. Flexible Job Shop Scheduling by Tabu Search. Technical Report Series ORP96-09, 1996.

BEHNKE, D.; GEIGER, M. J. Test instances for the flexible job shop scheduling problem with work centers. [S.l.]: Institut für betriebliche Logistik und Organisation, 2012.

BIRGIN, E. et al. A milp model for an extended version of the flexible job shop problem. Optimization Letters, Springer Berlin Heidelberg, v. 8, n. 4, p. 1417-1431, 2014.

BLUM, C.; ROLI, A. Metaheuristics in combinatorial optimization: Overview and conceptual comparison. ACM Computing Surveys (CSUR), ACM, v. 35, n. 3, p. 268-308, 2003.

BOSCHETTI, M. A. et al. Matheuristics: optimization, simulation and control. In: Hybrid metaheuristics. [S.l.]: Springer, 2009. p. 171-177.

BOWMAN, E. H. The schedule-sequencing problem. Operations Research, INFORMS, v. 7, n. 5, p. 621-624, 1959.

BRANDIMARTE, P. Routing and scheduling in a flexible job shop by tabu search. Annals of Operations Research, v. 41, n. 3, p. 157-183, 1993.

BRUCKER, P. An efficient algorithm for the job-shop problem with two jobs. Computing, Springer, v. 40, n. 4, p. 353-359, 1988. 
BRUCKER, P.; JURISCH, B.; SIEVERS, B. A branch and bound algorithm for the job-shop scheduling problem. Discrete applied mathematics, Elsevier, v. 49, n. 1, p. 107-127, 1994.

BRUCKER, P.; SCHLIE, R. Job-shop scheduling with multi-purpose machines. Computing, Springer, v. 45, n. 4, p. 369-375, 1990.

BRUCKER, P.; SCHLIE, R. Complex Scheduling. [S.l.]: Springer Verlag Berlin Heidelberg, 2006.

CARLIER, J. Ordonnancements a contraintes disjonctives. Revue française d'automatique, d'informatique et de recherche opérationnelle. Recherche opérationnelle, v. 12, n. 4, p. 333-350, 1978.

CARLIER, J. The one-machine sequencing problem. European Journal of Operational Research, Elsevier, v. 11, n. 1, p. 42-47, 1982.

CARLIER, J.; PINSON, E. An algorithm for solving the job-shop problem. Management science, INFORMS, v. 35, n. 2, p. 164-176, 1989.

CARLIER, J.; PINSON, E. A practical use of jackson's preemptive schedule for solving the job shop problem. Annals of Operations Research, v. 26, 1990.

CHAUDHRY, I. A.; KHAN, A. A. A research survey: review of flexible job shop scheduling techniques. International Transactions in Operational Research, Wiley Online Library, 2015.

CHEN, D.-S.; BATSON, R. G.; DANG, Y. Applied integer programming: modeling and solution. [S.l.]: John Wiley \& Sons, 2010.

CHENERY, H. B.; WATANABE, T. International comparisons of the structure of production. Econometrica: Journal of the Econometric Society, JSTOR, p. 487-521, 1958.

CLEMENTS, D. et al. Heuristic optimization: A hybrid ai/or approach. In: Workshop on Industrial Constraint-Directed Scheduling. [S.l.: s.n.], 1997.

CPLEX. IBM ILOG CPLEX Optimization Studio. 2016. Disponível em: <http: //www-01.ibm.com/software/integration/optimization/cplex-optimization-studio/>.

CPLEX-CP. IBM CPLEX CP Optimizer 12.6. 2016. urlhttp://www-01.ibm.com/software/commerce/optimization/cplex-cp-optimizer/.

CUNHA, A. G. et al. Manual de computação evolutiva e metaheurística. Imprensa da Universidade de Coimbra, 2012.

DANNA, E.; ROTHBERG, E.; PAPE, C. L. Exploring relaxation induced neighborhoods to improve mip solutions. Mathematical Programming, Springer, v. 102, n. 1, p. 71-90, 2005.

DAUZÈRE-PÉRÈS, S.; PAULLI, J. An integrated approach for modeling and solving the general multiprocessor job-shop scheduling problem using tabu search. Annals of Operations Research, Springer, v. 70, p. 281-306, 1997.

DEFERSHA, F.; CHEN, M. A parallel genetic algorithm for a flexible job-shop scheduling problem with sequence dependent setups. International Journal of Advanced Manufacturing Technology, v. 49, n. 1-4, p. 263-279, 2010. 
DEMIR, Y.; İŞLEYEN, S. K. Evaluation of mathematical models for flexible job-shop scheduling problems. Applied Mathematical Modelling, Elsevier, v. 37, n. 3, p. 977-988, 2013.

DOERNER, K. F.; SCHMID, V. Survey: matheuristics for rich vehicle routing problems. [S.l.]: Springer, 2010.

DOH, H.-H. et al. A priority scheduling approach for flexible job shops with multiple process plans. International Journal of Production Research, Taylor \& Francis, v. 51, n. 12, p. 3748-3764, 2013.

DUMITRESCU, I.; STÜTZLE, T. Combinations of local search and exact algorithms. In: Applications of Evolutionary Computing. [S.l.]: Springer, 2003. p. 211-223.

DYER, M. E.; WOLSEY, L. A. Formulating the single machine sequencing problem with release dates as a mixed integer program. Discrete Applied Mathematics, Elsevier, v. 26, n. 2, p. $255-270,1990$.

FATTAHI, P.; JOLAI, F.; ARKAT, J. Flexible job shop scheduling with overlapping in operations. Applied Mathematical Modelling, v. 33, n. 7, p. 3076-3087, 2009.

FATTAHI, P.; MEHRABAD, M. S.; JOLAI, F. Mathematical modeling and heuristic approaches to flexible job shop scheduling problems. Journal of Intelligent Manufacturing, v. 18, n. 3, p. 331-342, 2007.

FERNANDES, S.; LOURENÇO, H. R. Hybrids combining local search heuristics with exact algorithms. In: V Congreso Espanol sobre Metaheuristicas, Algoritmos Evolutivos y Bioinspirados. [S.l.: s.n.], 2007. p. 269-274.

FERNÁNDEZ, M. Á. G.; VELA, M. d. C. R.; ARIAS, J. R. V. An efficient memetic algorithm for the flexible job shop with setup times. In: ASSOCIATION FOR THE ADVANCEMENT OF ARTIFICIAL INTELLIGENCE (AAAI). Proceedings of the Twenty-Third International Conference on Automated Planning and Scheduling. [S.l.], 2013.

FERREIRA, C. E.; WAKABAYASHI, Y. Combinatória poliédrica e planos-de-corte faciais. Campinas, SP: Instituto de Computação. Universidade de Campinas, 1996.

FISCHETTI, M.; LODI, A. Local branching. Mathematical programming, Springer, v. 98, n. 1-3, p. 23-47, 2003.

FISCHETTI, M.; POLO, C.; SCANTAMBURLO, M. A local branching heuristic for mixed-integer programs with 2-level variables, with an application to a telecommunication network design problem. Networks, v. 44, n. 2, p. 61-72, 2004.

FISHER, H.; THOMPSON, G. L. Probabilistic learning combinations of local job-shop scheduling rules. p. 225-251, 1963.

FORTEMPS, P. Jobshop scheduling with imprecise durations: a fuzzy approach. Fuzzy Systems, IEEE Transactions on, IEEE, v. 5, n. 4, p. 557-569, 1997.

GAO, J.; GEN, M.; SUN, L. Scheduling jobs and maintenances in flexible job shop with a hybrid genetic algorithm. Journal of Intelligent Manufacturing, Springer, v. 17, n. 4, p. 493-507, 2006.

GAREY, M. R.; JOHNSON, D. S.; SETHI, R. The complexity of flowshop and jobshop scheduling. Mathematics of operations research, INFORMS, v. 1, n. 2, p. 117-129, 1976. 
GENDREAU, M.; POTVIN, J.-Y. Handbook of metaheuristics. [S.l.]: Springer, 2010.

GLOVER, F. Future paths for integer programming and links to artificial intelligence. Computers $\&$ operations research, Elsevier, v. 13, n. 5, p. 533-549, 1986.

GLOVER, F. W.; KOCHENBERGER, G. A. Handbook of metaheuristics. [S.l.]: Springer Science \& Business Media, 2006.

Gomez Morales, S. W. Formulações matemáticas e estratégias de resolução para o problema job shop clássico. Dissertação (Mestrado) — Escola Politécnica. Universidade de São Paulo, 2012. Disponível em: < http://www.teses.usp.br/teses/disponiveis/3/3136/tde-13062013-164920/>.

GOMORY, R. E. Outline of an algorithm for integer solutions to linear programs. Bull. Amer. Math. Soc., American Mathematical Society, v. 64, n. 5, p. 275-278, 091958.

GRABOWSKI, J.; NOWICKI, E.; ZDRZAŁKA, S. A block approach for single-machine scheduling with release dates and due dates. European Journal of Operational Research, Elsevier, v. 26, n. 2, p. 278-285, 1986.

GRAHAM, R. L. et al. Optimization and approximation in deterministic sequencing and scheduling: a survey. Annals of discrete mathematics, Elsevier, v. 5, p. 287-326, 1979.

GRÖTSCHEL, M.; JÜNGER, M.; REINELT, G. A cutting plane algorithm for the linear ordering problem. Operations research, INFORMS, v. 32, n. 6, p. 1195-1220, 1984.

GRÖTSCHEL, M.; JÜNGER, M.; REINELT, G. Facets of the linear ordering polytope. Mathematical Programming, Springer, v. 33, n. 1, p. 43-60, 1985.

GRÖTSCHEL, M.; LOVÁSZ, L.; SCHRIJVER, A. Geometric Algorithms and Combinatorial Optimization. Berlin: Springer, 1988.

GUROBI. Gurobi Optimizer Reference Manual. 2016. Disponível em: <http: //www.gurobi.com>.

HEFETZ, N.; ADIRI, I. An efficient optimal algorithm for the two-machines unit-time jobshop schedule-length problem. Mathematics of Operations Research, INFORMS, v. 7, n. 3, p. 354-360, 1982.

HOOKER, J. Toward unification of exact and heuristic optimization methods. International Transactions in Operational Research, Wiley Online Library, v. 22, n. 1, p. 19-48, 2015.

HURINK, J.; JURISCH, B.; THOLE, M. Tabu search for the job-shop scheduling problem with multi-purpose machines. Operations-Research-Spektrum, Springer, v. 15, n. 4, p. 205-215, 1994.

JACKSON, J. R. Scheduling a production line to minimize maximum tardiness. [S.l.], 1955.

JACKSON, J. R. An extension of johnson's results on job idt scheduling. Naval Research Logistics Quarterly, Wiley Online Library, v. 3, n. 3, p. 201-203, 1956.

JAIN, A. S.; MEERAN, S. Deterministic job-shop scheduling: Past, present and future. European journal of operational research, Elsevier, v. 113, n. 2, p. 390-434, 1999.

JOHNSON, S. M. Optimal two-and three-stage production schedules with setup times included. Naval research logistics quarterly, Wiley Online Library, v. 1, n. 1, p. 61-68, 1954. 
KACEM, I.; HAMMADI, S.; BORNE, P. Approach by localization and multiobjective evolutionary optimization for flexible job-shop scheduling problems. IEEE Transactions on Systems, Man and Cybernetics Part C: Applications and Reviews, v. 32, n. 1, p. 1-13, 2002.

KARP, R. M. Reducibility among combinatorial problems. In: Complexity of computer computations. [S.l.]: Springer, 1972. p. 85-103.

KHALIFE, M. A.; ABBASI, B.; ABADI, A. A simulated annealing algorithm for multi objective flexible job shop scheduling with overlapping in operations. Journal of Optimization in Industrial Engineering, v. 5, p. 17-28, 2010.

KIM, K.-H.; EGBELU, P. Scheduling in a production environment with multiple process plans per job. International Journal of Production Research, Taylor \& Francis, v. 37, n. 12, p. $2725-2753,1999$.

KLAU, G. W. et al. Combining a memetic algorithm with integer programming to solve the prize-collecting steiner tree problem. In: SPRINGER. Genetic and evolutionary computation-gecco 2004. [S.1.], 2004. p. 1304-1315.

LAND, A. H.; DOIG, A. G. An automatic method of solving discrete programming problems. Econometrica, v. 28, n. 3, p. 497-520, 1960.

LAWLER, E. L. et al. Sequencing and scheduling: Algorithms and complexity. Handbooks in operations research and management science, Amsterdam, v. 4, p. 445-522, 1993.

LAWRENCE, S. Supplement to resource constrained project scheduling: an experimental investigation of heuristic scheduling techniques. GSIA, Carnagie Mellon University, Pittsburg, PA, 1984.

LEFFLER, D. Y.-M. New valid inequalities for a time-indexed formulation of the flexible job shop scheduling problem. Dissertação (Mestrado) - Department of Mathematical Sciences, Chalmers University of Technology, 2015.

LEI, D. A genetic algorithm for flexible job shop scheduling with fuzzy processing time. International Journal of Production Research, v. 48, n. 10, p. 2995-3013, 2010.

LEI, D. Co-evolutionary genetic algorithm for fuzzy flexible job shop scheduling. Applied Soft Computing Journal, v. 12, n. 8, p. 2237-2245, 2012.

LENSTRA, J. K. Sequencing by enumerative methods. [S.1.]: Mathematisch centrum Amsterdam, 1976.

LENSTRA, J. K. Local search in combinatorial optimization. [S.l.]: Princeton University Press, 2003.

LENSTRA, J. K.; KAN, A. R. Complexity of scheduling under precedence constraints. Operations Research, INFORMS, v. 26, n. 1, p. 22-35, 1978.

LEUNG, J. Y. Handbook of scheduling: algorithms, models, and performance analysis. [S.1.]: CRC Press, 2004.

LI, J.-Q.; PAN, Q.-K.; TASGETIREN, M. A discrete artificial bee colony algorithm for the multi-objective flexible job-shop scheduling problem with maintenance activities. Applied Mathematical Modelling, v. 38, n. 3, p. 1111-1132, 2014. 
LIN, Z.-Z.; BEAN, J. C.; WHITE, C. C. A hybrid genetic/optimization algorithm for finite-horizon, partially observed markov decision processes. INFORMS Journal on Computing, INFORMS, v. 16, n. 1, p. 27-38, 2004.

LINDEROTH, J. T.; RALPHS, T. K. Noncommercial software for mixed-integer linear programming. Integer programming: theory and practice, CRC Press Operations Research Series, v. 3, p. 253-303, 2005.

MACAMBIRA, E. M. Uma abordagem de programação linear inteira para o problema de clique maxima com peso nas arestas. Dissertação (Mestrado) - Instituto de Computação. Universidade de Campinas, Campinas, SP, 2009.

MANNE, A. S. On the job-shop scheduling problem. Operations Research, INFORMS, v. 8, n. 2, p. 219-223, 1960.

MARTÍ, R.; REINELT, G. The linear ordering problem: exact and heuristic methods in combinatorial optimization. [S.1.]: Springer Science \& Business Media, 2011.

MASTROLILli, M. Flexible Job Shop Problem. http://people.idsia.ch/ monaldo/fjsp.html: [s.n.], 2016. Acessado em 01 de julho de 2016.

MASTROLILLI, M.; GAMBARDELLA, L. Effective neighbourhood functions for the flexible job shop problem. Journal of Scheduling, v. 3, n. 1, p. 3-20, 2000.

MAXIMA. Maxima, a Computer Algebra System. Version 5.34.1. 2014. Disponível em: $<$ http://maxima.sourceforge.net/ $>$.

MCMAHON, G.; FLORIAN, M. On scheduling with ready times and due dates to minimize maximum lateness. Operations research, INFORMS, v. 23, n. 3, p. 475-482, 1975.

MILIOTIS, P. Integer programming approaches to the travelling salesman problem. Mathematical Programming, v. 10, n. 1, p. 367-378, 1976.

MLADENOVIĆ, N.; HANSEN, P. Variable neighborhood search. Computers \& Operations Research, Elsevier, v. 24, n. 11, p. 1097-1100, 1997.

MUTH, J. F.; THOMPSON, G. L. Industrial Scheduling. [S.l.]: Prentice Hall, 1963.

NAGAR, A.; HERAGU, S.; HADDOCK, J. A meta-heuristic algorithm for a bi-criteria scheduling problem. Annals of Operations Research, v. 63, p. 397-414, 1995.

NAJID, N. M.; DAUZERE-PÉRÈS, S.; ZAIDAT, A. A modified simulated annealing method for flexible job shop scheduling problem. In: IEEE. Systems, Man and Cybernetics, 2002 IEEE International Conference on. [S.1.], 2002. v. 5, p. 6-pp.

NEMHAUSER, G.; SAVELSBERGH, M. W. A cutting plane algorithm for the single machine scheduling problem with release times. In: Combinatorial Optimization. [S.l.]: Springer, 1992. p. 63-83.

NOURALI, S.; IMANIPOUR, N.; SHAHRIARI, M. A mathematical model for integrated process planning and scheduling in flexible assembly job shop environment with sequence dependent setup times. International Journal of Mathematical Analysis, v. 6, n. 41-44, p. 2117-2132, 2012. 
OSMAN, I. H.; LAPORTE, G. Metaheuristics: A bibliography. Annals of Operations research, Springer, v. 63, n. 5, p. 511-623, 1996.

ÖZGÜVEN, C.; ÖZBAKIR, L.; YAVUZ, Y. Mathematical models for job-shop scheduling problems with routing and process plan flexibility. Applied Mathematical Modelling, Elsevier, v. 34, n. 6, p. 1539-1548, 2010.

ÖZGÜVEN, C.; YAVUZ, Y.; ÖZBAKIR, L. Mixed integer goal programming models for the flexible job-shop scheduling problems with separable and non-separable sequence dependent setup times. Applied Mathematical Modelling, Elsevier, v. 36, n. 2, p. 846-858, 2012.

PADBERG, M.; RINALDI, G. A branch-and-cut algorithm for the resolution of large-scale symmetric traveling salesman problems. SIAM review, SIAM, v. 33, n. 1, p. 60-100, 1991.

PALACIOS, J. et al. Genetic tabu search for the fuzzy flexible job shop problem. Computers and Operations Research, v. 54, p. 74-89, 2014.

PFETSCH, M. E. Branch-and-cut for the maximum feasible subsystem problem. SIAM Journal on Optimization, SIAM, v. 19, n. 1, p. 21-38, 2008.

PINEDO, M. L. Scheduling: theory, algorithms, and systems. [S.1.]: Springer Science \& Business Media, 2012.

POCHET, Y.; WOLSEY, L. A. Production planning by mixed integer programming. [S.l.]: Springer Science \& Business Media, 2006.

PUCHINGER, J.; RAIDL, G. R. Combining metaheuristics and exact algorithms in combinatorial optimization: A survey and classification. In: SPRINGER. International Work-Conference on the Interplay Between Natural and Artificial Computation. [S.1.], 2005. p. $41-53$.

PUCHINGER, J.; RAIDL, G. R. Combining (integer) linear programming techniques and metaheuristics for combinatorial optimization. In: Hybrid metaheuristics. [S.l.]: Springer, 2008. p. 31-62.

QUINTIQ. Quintiq - Supply Chain Planning and Optimization software. 2016. $<$ http://www.quintiq.com>. Acessado em 01/07/2016.

RAJKUMAR, M.; ASOKAN, P.; VAMSIKRISHNA, V. A grasp algorithm for flexible job-shop scheduling with maintenance constraints. International Journal of Production Research, v. 48, n. 22, p. 6821-6836, 2010.

RAYWARD-SMITH, V. J. et al. Modern heuristic search methods. [S.l.]: Wiley New York, 1996.

RESULTS-QUINTIQ. Results: Flexible Job Shop Scheduling Problem - Quintiq. 2016. < http: //www.quintiq.com/optimization/flexible-job-shop-scheduling-problem-results.html $>$. Acessado em 01/07/2016.

RIBEIRO, G. F.; LORENA, L. A. N. Constructive genetic algorithm and column generation: an application to graph coloring. In: Proceedings of APORS. [S.l.: s.n.], 2000.

ROSSI, A. Flexible job shop scheduling with sequence-dependent setup and transportation times by ant colony with reinforced pheromone relationships. International Journal of Production Economics, v. 153, p. 253-267, 2014. 
ROSSI, A.; DINI, G. Flexible job-shop scheduling with routing flexibility and separable setup times using ant colony optimisation method. Robotics and Computer-Integrated Manufacturing, v. 23, p. 503-516, 2007.

ROY, B.; SUSSMANN, B. Les problemes de ordonnancement avec contraintes disjonctives. Note D.S., v. 9, 1964.

SANTOS, R. F. dos. Uso de cortes canônicos no método de ramificação local para problemas inteiros 0 e 1 mistos. Dissertação (Mestrado) - Instituto de Computação. Universidade de Campinas, 2006.

SCHUTT, A.; FEYDY, T.; STUCKEY, P. J. Scheduling optional tasks with explanation. In: SPRINGER. International Conference on Principles and Practice of Constraint Programming. [S.1.], 2013. p. 628-644.

STAGGEMEIER, A. T. et al. A hybrid genetic algorithm to solve a lot-sizing and scheduling problem. In: CITESEER. Proceedings of the 16th triannual Conference of the International Federation of Operational Research Societies, Edinburgh, UK. [S.l.], 2002.

TALBI, E.-G. Metaheuristics: from design to implementation. [S.l.]: John Wiley \& Sons, 2009.

THÖRNBLAD, K. et al. A competitive iterative procedure using a time-indexed model for solving flexible job shop scheduling problems. [S.l.]: Department of Mathematical Sciences, Division of Mathematics, Chalmers University of Technology and University of Gothenburg, 2013.

VOSE, M. D. The simple genetic algorithm: foundations and theory. [S.l.]: MIT press, 1999.

WAGNER, H. M. An integer linear-programming model for machine scheduling. Naval Research Logistics Quarterly, Wiley Online Library, v. 6, n. 2, p. 131-140, 1959.

WANG, S.; YU, J. An effective heuristic for flexible job-shop scheduling problem with maintenance activities. Computers and Industrial Engineering, v. 59, n. 3, p. 436-447, 2010.

WILLIAMS, H. P. Model building in mathematical programming. [S.l.]: John Wiley \& Sons, 2013.

WOLSEY, L. A.; NEMHAUSER, G. L. Integer and combinatorial optimization. [S.1.]: John Wiley \& Sons, 1988.

WOODRUFF, D. L. A chunking based selection strategy for integrating meta-heuristics with branch and bound. In: Meta-Heuristics. [S.l.]: Springer, 1999. p. 499-511.

XPRESS. FICO Xpress Optimization Suite. 2016. Disponível em: <http://www.fico.com/br/ products/fico-xpress-optimization-suite $>$.

YU, X.; RAM, B. Bio-inspired scheduling for dynamic job shops with flexible routing and sequence-dependent setups. International Journal of Production Research, v. 44, n. 22, p. 4793-4813, 2006.

ZABALA, P. Problemas de Ruteo de Vehículos. Tese (Doutorado) - Departamento de Computacion, Facultad de Ciencias Exactas y Naturales de la Universidad de Buenos Aires, 2006 . 